\title{
ASPECTOS MATEMÁTICOS (Y CONCEPTUALES) DE LA ECONOMÍA DE PIERO SRAFFA. DESARROLLOS
}

\author{
Antonio Mora Plaza \\ Economista, Madrid
}

https://doi.org/10.33676/EMUI_nomads.57.10

\section{Matematical and conceptual aspects of the economy of Piero Sraffa}

\begin{abstract}
Although the author of this work has published many works on the Piero Sraffa'work, this long article is dedicated specifically to the mathematics contained in his capital book, throughout his book, chapter by chapter. Such an effort can be justified because there is nothing similar in Spanish lenguage and because, as we will see, it may be allow us to clarify, to rectify and to develop, although limitedly, the mathematical content. The purpose is that it serves for the reading of Production of commodities by means of commodities, for to be able to enjoy its analytical economic content and also not to get lost with, sometimes, the disguised mathematical ramblings, sometimes also, of economic reasonings of the great turinés. Although the opposite is normal, the economist Italian's effort to build an economics book where mathematics seems to remain hidden is extraordinary. Of course, the particular use of Sraffa is updated to the current mathematical signs. In the present text, we have tried to be as original as possible so as not to repeat the inevitable partial aspects of previous works. In particular, this has been the case about the fixed capital and partially with the issues of reducing capital to dated work and joint production. On the rest has been tried to find some original elements. Hovewer the previuos comments, some of the conceptual contents have inevitable been included. The reason for this is that without understanding, critizing or devoloping them, the formal aspects can not be understood.
\end{abstract}

Keywords: Sraffa, economy, analysis

\section{Introducción}

Aunque el autor de este trabajo ha publicado muchos trabajos sobre la obra de Piero Sraffa, este largo artículo está dedicado, específicamente, a las matemáticas contenidas en su libro capital, en todo su libro, capítulo a capítulo. Tal esfuerzo se puede justificar porque no hay nada parecido a esto en español y porque, como veremos, nos va a permitir aclarar, rectificar y desarrollar, aunque sea limitadamente, el contenido matemático y conceptual. El propósito es que sirva para la lectura de Producción de mercancías por medio de mercancías, para poder disfrutar de su contenido económico analítico y no perderse con, a veces, las divagaciones matemáticas disfrazadas, a veces también, de razonamientos económicos del genial turinés. Aunque lo normal es lo contrario y resulta extraordinario el esfuerzo del italiano en construir un libro de economía donde las matemáticas parecen disfrazarse de noviembre. Por su puesto que se actualiza a los signos matemáticos actuales el uso particular que emplea Sraffa. Se ha procurado en el presente texto ser lo 
más original posible para no repetir los aspectos parciales inevitables de trabajos anteriores. En especial ha sido así en el tema del capital fijo y parcialmente en los temas de la reducción del capital a trabajo fechado y la producción conjunta. En el resto se ha procurado buscar algún elemento de originalidad, y, por supuesto, en relación con otros autores. No obstante lo anterior, inevitablemente se ha entrado en algunos de los contenidos conceptuales puesto que sin entenderlos, criticarlos si es menester o desarrollarlos, aunque sea limitadamente, no se puede entender los aspectos formales, tantos los explícitos como los implícitos, que hay en Producción de mercancías por medio de mercancías. Además y creo esperable que, para los estudiosos de la historia del análisis económico, entendiendo la ontogénesis del libro se pueda mejor investigar sobre su filogénisis, y más teniendo en cuenta su larga gestación (casi cuatro décadas).

En 1960 se publicaba, ipor fin!, el libro de Piero Sraffa Producción de mercancías por medio de mercancías!. Libro de larga gestación porque el propio Sraffa nos dice en el prólogo que "... en 1928, lord Keynes, leyó un borrador de las primeras proposiciones...". La obra de Sraffa en su conjunto solo consideramos sus artículos de 1925 y 1926 y su obra capital- es una obra revolucionaria en lo intelectual, obra que se presentaba y se presenta inevitablemente como una alternativa al marginalismo dominante en su época, con los Principios de Economía (los Principles, de Alfred Marshall, 18421924) como la obra canónica de economía, donde se formaban tanto los profesores como los alumnos de Economía a partir de la última década del siglo XIX hasta bien entrada la tercera década del siglo XX. La obra de Sraffa tiene de común con la de otro importante economista de la misma época como es el análisis Input-Ouput (Leontief) dos aspectos: ambas inciden más o menos explícitamente en la importancia de las relaciones intersectoriales compra-venta entre empresas- y en el uso del álgebra como formalismo matemático. Especialmente del álgebra matricial, aunque con una diferencia entre ambos porque, si bien en Leontief el uso de las matemáticas se hace explícita e inevitable, en la obra de Sraffa se hace un esfuerzo denodado por esconder en lo posible el formalismo, para que no pareciera que se trataba de un libro de matemáticas aplicado a la Economía y sí un libro de Economía que usa ese maravilloso instrumento, más descubierto que inventado por la humanidad. Aún así y como veremos a lo largo de estas páginas, su uso también se hace inevitable y con más razón que con Leontief. Ahora y desde hace algunas décadas no se cuestiona la necesidad del uso de las matemáticas en el análisis económico -y también de los gráficos-, pero no fue así en épocas anteriores². De ahí también y no sólo por los contenidos por lo que la obra de Sraffa es revolucionaria. Pero el contenido matemático de Sraffa adolece de algunos defectos por dos motivos principalmente: porque Sraffa no era precisamente un gran matemático y por ese esfuerzo comentado por evitar que su libro pudiera ser meramente entendido como un libro de

\footnotetext{
1 Se publicaba en inglés con el nombre de Production of Commodities by means of Commodities.

2 Otra cosa es lo que hacía Joan Robinson con sus alumnos, a los que obligaba a explicar el análisis económico sin gráficos para cerciorarse que lo habían entendido.
} 
matemáticas aplicadas a la Economía, cosa que ocurrió con el modelo matemático de John von Neumann, por poner un ejemplo. Sin embargo hay que decir que Sraffa demuestra en su libro entender las matemáticas -al menos el álgebra-, pero que parecía desconocer algunas propiedades del álgebra matricial como es el teorema de Perron-Frobenius o el de Rouché-Frobenius ${ }^{3}$, aunque quizás conociera los teoremas del punto fijo. De lo contrario no podría haber mostrado esa seguridad en la existencia de un vector de precios positivo para la producción simple, por ejemplo. Señalar que Sraffa estuvo magníficamente asesorado por matemáticos ${ }^{4}$ de máximo nivel de su época como fueron Frank Ramsey, A. S. Besicovitch y Alister Watson, a los que menciona en el prólogo de su libro y agradece su ayuda ${ }^{5}$. Este artículo trata de esclarecer algunos de los problemas matemáticos que tiene y contiene Producción de mercancías por medio de mercancías y que añade dificultad a la lectura del libro. Iremos en lo posible, capítulo a capítulo, siguiendo, esclareciendo y desarrollando en los aspectos formales -pero también en los contenidos económicos- su obra con el fin de ayudar a su lectura; también para aclarar y rectificar algún error que comete. Pero, como se verá, este trabajo es también una exposición, aclaración y desarrollo -en este caso muy limitado- del genial libro del economista turinés, de sus aspectos conceptuales y estructurales. No puede ser menos si se concibe las matemáticas siempre como un instrumento del conocimiento y ayuda de aspectos de los análisis -del tipo que sean-susceptibles de ser formalizables. Y a pesar de lo dicho, el libro de Sraffa y su obra en su conjunto son, a juicio modestísimo del autor de este trabajo, una de las grandes obras intelectuales del siglo XX. Eso no quita para que en España Sraffa sea realmente un desconocido. Es verdad que se le menciona en muchas ocasiones pero siempre de pasada, sin profundizar apenas en su obra, sin que su obra forme parte del corpus de conocimientos de un economista. Y aún menos existen desarrollos de su obra. Al menos eso es así en castellano 6 . Y fuera de España se han centrado generalmente más en los

\footnotetext{
3 En el caso del teorema de Rouché-Frobenius es posible que no conociera la formulación exacta del teorema, pero sabía y entendía perfectamente cuando un sistema de ecuaciones tiene una, ninguna o infinitas soluciones. Por otro lado es también un conocimiento elemental y universal de las matemáticas. En el caso del teorema de Perron-Frobenius no da muestras en el libro de conocerlo, sino todo lo contrario, cuando aborda el tema de la mercancía-patrón. Parece un tema abierto a las posibles investigaciones sobre sus notas y correspondencia.

${ }_{4}^{4}$ Salvando las diferencias de todo tipo, este hecho recuerda las dificultades de A. Einstein con su teoría generalizada de la relatividad y la impagable ayuda que recibió del matemático y amigo Marcel Grossmann por el dominio que tenía éste de la matemática de tensores.

${ }_{5}$ Sobre el conocimiento que tenía Piero Sraffa de las matemáticas, de cómo las fue usando y desechando, sobre si hizo caso o no, o solo a veces a los magníficos matemáticos que le asesoraron, está pendiente del esfuerzo que puedan hacer los estudiosos de Sraffa sobre estos temas y sobre, al parecer, los infinitos temas sobre los que estudió y meditó Sraffa. Su correspondencia, sus miles de cartas, sus notas, están depositadas en el Trinity College de la Universidad de Cambridge. H. D. Kurz dio un pequeño avance de lo que se puede investigar, aclarar de los escritos de Sraffa en su artículo traducido por U. Autónoma del Estado de México Las Contribuciones de Sraffa a la Economía. Algunas notas sobre sus trabajos inéditos. Fue publicado en castellano en el 2007, pero la primera versión, según nota del propio artículo, lo fue en italiano en 1998. En realidad está por hacer parecido esfuerzo al que hizo Sraffa con su admirado David Ricardo.

6 De ahí también la dificultad de encontrar evaluadores de artículos sobre Sraffa que tengan un conocimiento mínimo para tal tarea. A Sraffa se le puede llegar a entender con años trabajando su obra y, aun así, siempre su lectura nos depara sorpresas y nuevos caminos.
} 
aspectos matemáticos que conceptuales. Además con un defecto y es el de anteponer las matemáticas a la economía, a poner condiciones matemáticas antes que asentar las hipótesis económicas o bien se ha hecho historia del análisis económico. Eso ha sido así en libros magníficos -desde otros puntos de vista- como los de Kurz, Steedman, Schefold, Frois-Berrebi, etc. Pasinetti creo que ha sido una excepción con su análisis vertical integrado. Decía que generalmente porque mencionamos en la bibliografía, por ejemplo, una recopilación de artículos entre otros de Kurz, Schefold, Salvadori, etc. es Sraffa or an alternative economics, del 2008. Hay más excepciones, desde luego. Entramos sin más dilación en materia, siguiendo capítulo a capítulo Producción de mercancías por medio de mercancías. Se ha procurado ser sintético -quizá demasíado-por falta de espacio, pero es de esperar que sea suficiente para el fin que se propone: ayudar a la comprensión del texto de Sraffa a partir de la comprensión de los aspectos formales.

\section{Capítulo I - PRODUCCIÓN DE SUBSISTENCIA}

Parte Sraffa en este capítulo de un modelo extremadamente simple en el que se produce dos mercancías -hoy diríamos bienes y servicios- como son hierro y trigo, con dos medios que son también hierro y trigo. Además no hay excedente, lo cual significa que la suma del hierro empleado en la producción de ambos bienes es igual al hierro producido e, igualmente, pasa con el trigo. Lo mismo ocurre cuando extiende Sraffa su modelo a tres bienes. Hasta aquí no hay problema matemático digno de mención. El sistema de ecuaciones pertinente sería:

$$
y_{i}=\sum_{j=1}^{j=n} x_{i j} \quad \text { para todo } i=1 \text { a } \quad n
$$

siendo $\mathbf{x}_{\mathrm{ij}}$ la cantidad de la mercancía $\boldsymbol{i}$ que procede del sector o proceso ${ }^{7} \boldsymbol{j}$.

\footnotetext{
7 Definitivamente hay que inscribir siempre la procedencia o destino de las mercancías de procesos más que de sectores. Esta es una de las grandes diferencias del modelo de Sraffa (procesos) con el de Leontief (sectores).
} 
El primer problema surge cuando Sraffa introduce los precios para poder sumar los medios de producción que concurren en la producción de cada uno de los bienes. Entonces tenemos un sistema de $\boldsymbol{n}$ ecuaciones con $\boldsymbol{n}$ incógnitas tal como:

$$
p_{i} y_{i j}=\sum_{i=1}^{i=n} p_{i} x_{i j} \text { para todo } j=1 \text { a n y con } y_{i j}=0 \text { si } i<>j
$$

Y ahora se plantea si tiene solución el sistema. Sraffa lo aborda desde el punto de vista de un economista y considera que no hay las $\boldsymbol{n}$ incógnitas aludidas sino $\mathbf{n}-\mathbf{1}$, dado que sólo hay $\mathbf{n}-\mathbf{1}$ precios relativos, porque uno de los precios debe considerarse como un numerario. En la práctica eso supone dividir todas las ¡ecuaciones?! entre uno de los precios. Pero, dado que tenemos $\boldsymbol{n}$ ecuaciones, una de estas ha de eliminarse porque Sraffa tiene claro que un sistema de ecuaciones solo tiene solución única si el número de ecuaciones y de incógnitas coinciden?. Sobre este punto hay que decir dos cosas: que lo anterior es la condición necesaria pero que hay que complementarla con la condición de que ninguna de las ecuaciones -que son lineales- sea combinación lineal de las otras. Y decir también que el teorema-el teorema de Rouché-Frobenius ${ }^{10}$ - permite esclarecer todo esto. Sin embargo la solución de Sraffa no parece aceptable porque: ¿̇qué ecuación eliminamos? Una solución es calcular los precios absolutos, las $\boldsymbol{n}$ ecuaciones que surgen del sistema de $\boldsymbol{n}$ ecuaciones $y$, a posteriori, dividir las $\boldsymbol{n}$ precios entre uno cualesquiera o dividirlos, por ejemplo, entre el producto nacional dado por el escalar PYI, con lo cual tendremos precios relativos o precios en función de este producto. También puede emplearse como divisor de todas las ecuaciones -es decir, como numerario- el excedente en términos monetarios que viene dado por la expresión $\mathbf{P}(\boldsymbol{Y}-\boldsymbol{X}) \mathbf{I}$. Además, en el caso primero, es decir, en el caso de que se dividan los precios obtenidos a posteriori, lo que tenemos son precios que son también índices de intercambio entre mercancías y que lleva a una economía a un doble equilibrio: equilibrio en términos físicos porque el total de medios de producción de cada mercancía es igual al producto final de cada una de ellas, y equilibrio en términos de valor, porque la suma del total de las mercancías valoradas a sus precios es igual a la suma, en términos de valor, de

\footnotetext{
8 Acentúo que lo que se divide son todas las ecuaciones y no solo los precios contenidos en ellas, como podría interpretarse leyendo a Sraffa. Por esta razón no estoy seguro de que el economista italiano entendiera de forma correcta el aspecto matemático de lo que es un numerario.

9 Si ninguna ecuación es combinación lineal de las otras. Es una versión reducida del teorema Rouché-Frobenius.

10 En el libro de Luigi Pasinetti Lecciones de teoría de la producción se recoge un excelente apéndice matemático de álgebra matricial aplicado a la Economía. El libro de Pasinetti debiera ser un libro de cabecera de todos los economistas, fuera cual fuera la escuela económica a la que se sientan más concernidos. En concreto, las condiciones del teorema de Roché-Frobenius se recogen en las páginas 328 (epígrafe 8.5) y siguientes del libro mencionado.
} 
todas las mercancías (hoy, bienes y servicios) que entran como medios de producción:

$$
\sum_{i=1}^{n} \sum_{j=1}^{n} p_{i} y_{i j}=\sum_{i=1}^{n} \sum_{j=1}^{n} p_{i} x_{i j} \quad \text { con } y_{i j}=0 \text { si } i<>j
$$

El segundo problema que provoca la introducción de los precios es asegurar su positividad, pero Sraffa no hace mención en este capítulo de esta dificultad. La ecuación (1.2) tiene su formulación matricial como:

$$
P Y=P X
$$

donde $\boldsymbol{P}$ es un vector vertical de $\mathbf{1}$ a $\boldsymbol{n}, \boldsymbol{X}$ es una matriz $\boldsymbol{n} \times \boldsymbol{n}$ de $\boldsymbol{n} \times \boldsymbol{n}$ elementos e $\boldsymbol{Y}$ es una matriz $\boldsymbol{n} \times \boldsymbol{n}$ pero de $\boldsymbol{n}$ elementos, puesto que es una matriz diagonal. A esta ecuación matricial añadimos la de coeficientes técnicos $\boldsymbol{A} n \times n$, que viene dada por:

$$
X=A Y
$$

Que, sustituida en (1.4), queda:

$$
P[I-A] Y=0
$$

donde cada elemento de la matriz $\boldsymbol{A}$ representa la cantidad del bien $\mathbf{x}_{\mathrm{ij}}$ necesaria para producir una unidad del bien $\boldsymbol{y}_{\mathrm{j}}$. También (1.4) puede ser puesto como:

$$
P=P X Y^{-1}
$$

y donde $X Y^{-1}=\boldsymbol{A}$. En (1.6) no podemos asegurar que, sean cuales sean los elementos de la matriz $\boldsymbol{A}$, exista un vector de precios estrictamente positivo, pero sí se puede asegurar que si $\boldsymbol{A}$ es una matriz cuadrada, positiva e indescomponible (irreducible) ${ }^{11}$ y si, además, el autovalor máximo por la izquierda es igual a 1 -lo cual implica que no hay excedente, como es el caso-, entonces dicho autovalor nos da un vector de precios estrictamente positivo. Es decir, se podría aplicar el teorema de Perron-Frobenius aunque para el caso particular de que no haya excedente. Veamos un ejemplo. Sea un modelo de

${ }^{11}$ Más adelante daremos alguna explicación más extensa del teorema. 
producción de subsistencia como el que establece el propio Sraffa en su libro (capítulo I, epígrafe 2) como sigue:

\begin{tabular}{|c|c|c|c|c|}
\hline & \multicolumn{3}{|c|}{$Y=$ productos finales } & \multirow[b]{2}{*}{ 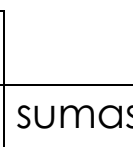 } \\
\hline & proceso 1 & proceso 2 & proceso 3 & \\
\hline \multirow{5}{*}{$\begin{array}{l}\text { trigo } \\
\text { hierro } \\
\text { cerdos }\end{array}$} & 450 & 0 & 0 & 450 \\
\hline & 0 & 21 & 0 & 21 \\
\hline & 0 & 0 & 60 & 60 \\
\hline & & $\begin{array}{l}\mathrm{X}= \\
\text { medios }\end{array}$ & & \\
\hline & proceso 1 & proceso 2 & proceso 3 & sumas \\
\hline \multirow{5}{*}{$\begin{array}{l}\text { trigo } \\
\text { hierro } \\
\text { cerdos }\end{array}$} & 240 & 90 & 120 & 450 \\
\hline & 12 & 6 & 3 & 21 \\
\hline & 18 & 12 & 30 & 60 \\
\hline & inputs de & trabajo & 3. & \\
\hline & trigo & hierro & cerdos & total \\
\hline$L=$ & 18,0 & 12,0 & 30,0 & 60,0 \\
\hline
\end{tabular}

Con este sistema donde no hay excedente no es posible obtener directamente ${ }^{12}$ el vector de precios de la ecuación matricial $\mathbf{P Y}=\mathbf{P X}$, razón por la cual hemos recurrido a la programación lineal. Hemos supuesto que la $\underline{\text { función objetivo }}^{13}$ sea $P Y I=1$, que las variables a determinar sean los tres precios absolutos $\boldsymbol{P}$ y que la restricción sea la ecuación matricial $\mathbf{P Y}=\boldsymbol{P X}$. El resultado de esta es:

\begin{tabular}{|l|l|l|l|l|}
\cline { 2 - 4 } \multicolumn{1}{c|}{} & trigo & hierro & cerdos & PYI \\
\hline & 0,4687 & 0,2187 & 0,3125 & 1,0000 \\
\hline & & & & \\
\cline { 2 - 4 } & & &
\end{tabular}

12 No se puede obtener mediante procesos elementales del álgebra, aunque sí lo podemos hacer mediante algún algoritmo, como es el caso de la programación lineal -que aquí utilizamos- porque el método del simplex busca, de entre todas las soluciones factibles, la óptima a base de dar soluciones provisionales a subconjuntos del sistema de igual número de ecuaciones que de incógnitas y eliminando las variables que más se alejan de la función objetivo y permitiendo la entrada que más se le acercan. No es el caso con el modelo de Sraffa porque entran en la evaluación todas las variables, dado que estas son iguales al número de ecuaciones, por lo que solo queda la evaluación de lo que aporta cada variable en aras de acercarse a la función objetivo.

13 Lo cual supone a la vez tomar a PYI como numerario. 


\begin{tabular}{|c|c|c|c|c|}
\hline$\left(1+g_{m}\right) P X=$ & 0,4687 & 0,2187 & 0,3125 & 1,0000 \\
\hline
\end{tabular}

Y el resultado final es el vector horizontal de precios:

\begin{tabular}{|l|l|l|l|}
\cline { 2 - 4 } \multicolumn{1}{c|}{} & trigo & hierro & cerdos \\
\hline precios absolutos $=$ & 0,00104 & 0,01042 & 0,00521 \\
\hline
\end{tabular}

Los precios relativos se obtienen a posteriori, dividiendo, por ejemplo, el precio unitario del hierro y el de cerdos entre el precio del trigo. El resultado:

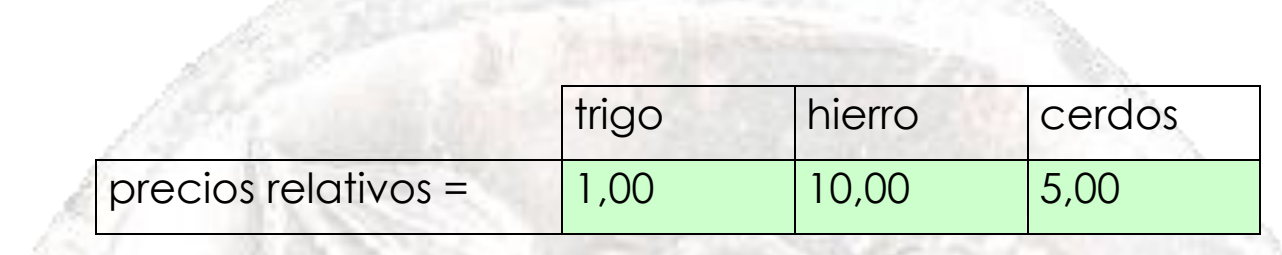

Estos últimos precios son propiamente las unidades que se intercambiarán entre sí el trigo, hierro y cerdos para que el sistema esté en un estado de autoreemplazamiento, tal como dice y hace Sraffa. Pero la novedad en el cálculo que hemos empleado es la de que los precios relativos los hemos obtenido a posteriori, es decir, una vez que tuvimos los precios absolutos.

II - Una última reflexión en este capítulo es la posibilidad de la programación lineal. La programación lineal del método simplex nos da una solución en este caso al conjunto de $\boldsymbol{n}$ ecuaciones con $\boldsymbol{n}$ incógnitas que representa $\boldsymbol{P Y}=\boldsymbol{P X}$. $\boldsymbol{Y}$, en este caso $-\mathrm{y}$ dado que el vector de precios $\boldsymbol{P}$ es el mismo que pre-multiplica a las matrices de productos $\boldsymbol{Y}$ y de medios $\boldsymbol{X}$ - estamos en un caso de aplicación del teorema del punto fijo, donde $\boldsymbol{P}$ es el espacio de soluciones (lo suponemos definido en el espacio de los números reales). El conjunto de soluciones factibles es único dado que tenemos igual número de ecuaciones que de incógnitas. Todas estas condiciones avocan a que el método de programación lineal pueda ser considerado como un caso particular de la aplicación de los teoremas del punto fijo. En realidad el método simplex de programación es un algoritmo de búsqueda de un punto fijo de una función continua (cualesquiera de las de Sraffa así la consideramos por hipótesis) aplicada en un conjunto (el de los precios) que entendemos como cerrado, acotado y convexo. 


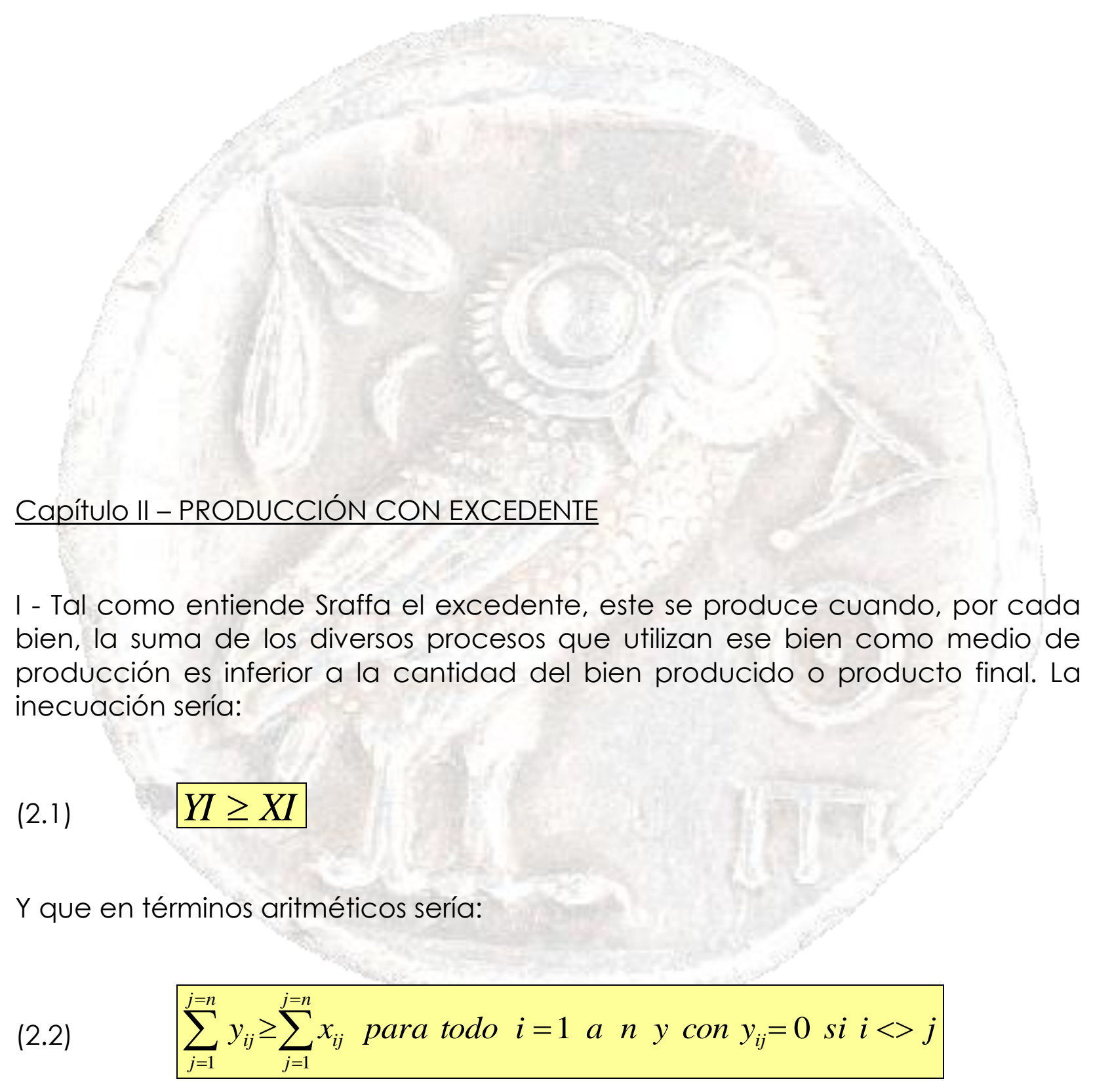

No contempla Sraffa la posibilidad de que, para algunos de los bienes, la producción final fuera inferior a la suma de los medios empleados de ese bien en el conjunto de la economía. Obsérvese que lo que se dice, no es que la suma de los medios empleados en términos físicos (causa) en la producción de un bien sea superior al bien producido (efecto), sino que, para cada bien o servicio, jel total de los producido (para el conjunto de la economía)! tiene que 
ser mayor que los medios utilizado de ese bien o servicio jen el conjunto de la economía! Si el excedente físico anterior se traduce, además, en términos de valor y queda:

$$
\sum_{i=1}^{i=n} p_{i} y_{i j} \geq \sum_{i=1}^{i=n} p_{i} x_{i j} \quad \text { para todo } j=1 \text { a } n \text { y con } y_{i j}=0 \text { si } i<>j
$$

que en términos de álgebra matricial sería:

$$
\underset{1 x n}{P} \underset{n x n}{Y} \geq \underset{1 x n \text { xon }}{X}
$$

Esta desigualdad -y a diferencia del modelo de Neumann-, Sraffa establece que será la misma para todos los bienes producidos. Es decir, Sraffa traduce las diversas diferencias entre medios y productos en términos físicos para cada bien en Producción de mercancías por medio de mercancías en una única tasa que va a constituir la tasa máxima de ganancia ${ }^{14}$. La llamaremos $\mathbf{g}_{\mathrm{m}}$. Es verdad que Sraffa la llama $\boldsymbol{R}$, que es además la razón-patrón de la mercancíapatrón que desarrollará en otros capítulos, pero en todo esto el gran economista turinés se adelanta porque supone que ambas tasas son iguales sin demostrarlo nunca. Como adelanto diremos que, en efecto, ambas tasas son iguales en la producción simple, que es la producción que estamos considerando y que consiste que cada empresa produce un único producto y que cada producto es producido por una sola empresa. Eso implica que la matriz $Y$ de productos finales sea una matriz diagonal, razón por la cual se ha considerado en las ecuaciones que definen el sistema que $\boldsymbol{y}_{\boldsymbol{i j}}$ valdrá cero si $\boldsymbol{i}$ es diferente de $\boldsymbol{j}$. Pues bien, la ecuación que definiría entonces la producción con excedente en términos de valor ${ }^{15}$ sería en términos matriciales:

$$
\underset{1 x n \text { xn }}{P}=\left(1+g_{m}\right) \underset{1 x n \text { nxn }}{P} \underset{X}{X}
$$

O bien:

\footnotetext{
14 Matizar que Sraffa hace igual la tasa máxima de ganancia con la razón-patrón de la mercancía-patrón. Lo cual es cierto si no nos movemos de la producción simple, producción que tiene su traducción matemática en que la matriz de productos finales $Y$ sea una matriz diagonal.

15 Ahora, al definir la economía en ecuaciones en términos de valor, sí que hay que entender una relación causa y efecto entre los costes dados por la suma de vectores $\mathbf{W L}+\mathbf{P X}$ y el valor del producto final $\mathbf{P Y}$. Es decir, cada ecuación matricial $\mathbf{P Y}=(\mathbf{1}+\mathbf{g})[\mathbf{w L}+\mathbf{P X}]$ representa $\mathbf{n}$ ecuaciones (una por cada bien o servicio), en cada una de las cuales se establece una relación causa y efecto entre los medios materiales empleados dados por $\mathbf{x}_{\mathbf{i j}}$ para todos los $\mathbf{j}=\mathbf{1}$ a $\mathbf{n}$ más el trabajo empleado en la producción del bien $\mathbf{l}_{\mathbf{i}}$, con su efecto, que es $\mathbf{y}_{\mathrm{ij}}$, para todo $\mathbf{i}$ y para todo $\mathbf{j}$ concretos (esto último si estamos en la producción simple).
} 


$$
\begin{gathered}
p_{1} y_{1}=\left(1+g_{m}\right)\left[p_{1} x_{11}+p_{2} x_{21}+\ldots .+p_{n} x_{n 1}\right] \\
p_{2} y_{2}=\left(1+g_{m}\right)\left[p_{1} x_{12}+p_{2} x_{22}+\ldots .+p_{n} x_{n 2}\right] \\
\ldots \ldots \ldots \ldots \ldots \ldots \ldots \ldots \ldots \ldots \ldots \ldots \ldots \ldots \ldots \ldots \ldots \ldots \ldots \ldots \ldots \ldots \ldots \ldots \ldots \ldots \ldots \ldots \ldots \ldots \ldots \ldots \ldots \\
p_{n} y_{n}=\left(1+g_{m}\right)\left[p_{1} x_{1 n}+p_{2} x_{2 n}+\ldots . .+p_{n} x_{n n}\right]
\end{gathered}
$$

Del sistema de ecuaciones anterior obtenemos que el excedente en términos monetarios por proceso $\boldsymbol{j}$ vendría dado por el vector horizontal:

$$
\text { Excedente }=g_{m} P X
$$

En términos aritméticos sería:

$$
\text { Excedente de } j=g_{m} \sum_{i=1}^{i=n} p_{i} x_{i j} \text { para todo } j=1 \text { a } n
$$

Y que globalmente viene dado por:

$$
\text { Excedente }=g_{m} \sum_{i=1}^{i=n} \sum_{j=1}^{j=n} p_{i} x_{i j}
$$

Hasta aquí no hemos hecho sino seguir a Sraffa con la muleta del álgebra matricial y ésta nos ha servido para asentar sin dificultad lo dicho por el economista italiano en su obra magna, magna no por extensa sino por importante y original. Es verdad que Sraffa no menciona el teorema de Rouché-Frobenius para precisar sin dificultad cuando un sistema de ecuaciones tiene solución. El teorema nos dice que un sistema de $\boldsymbol{n}$ ecuaciones lineales de $\boldsymbol{n}$ incógnitas tiene solución única si coincide que el rango de la matriz original del sistema es igual al rango de la matriz ampliada y ambos son iguales a su vez al número de incógnitas. Aclaramos lo del rango original y ampliado. Por ejemplo, un sistema de $\boldsymbol{n}$ ecuaciones lineales dado por $\boldsymbol{A} \boldsymbol{Y}=\boldsymbol{D}$, donde $\boldsymbol{A}$ es una matriz $\boldsymbol{n} \times \boldsymbol{n}$, donde $\boldsymbol{D}$ e $\boldsymbol{Y}$ son vectores $\boldsymbol{n} \times \mathbf{1}$ e $\boldsymbol{Y}$ es matriz diagonal de $\boldsymbol{n}$ incógnitas, tendrá solución si el rango de la matriz $\boldsymbol{A}$ es igual al rango de la matriz ampliada $(\boldsymbol{A}, \boldsymbol{D})$ y ambos rangos son iguales a $\boldsymbol{n}$. Hecha la salvedad de que Sraffa no menciona el teorema, no tiene o no tendría más importancia si Sraffa empleara un uso correcto del concepto de numerario. Dicho de otra forma, en mi opinión Sraffa tenía clara la idea de que un sistema de $\boldsymbol{n}$ ecuaciones tiene solución 
única para $\boldsymbol{n}$ incógnitas ${ }^{16}$, pero hizo un mal uso del concepto de numerario porque se vio obligado a eliminar una ecuación del conjunto para cumplir el requisito anterior. Así, al partir en el capítulo I del sistema de $\boldsymbol{n}$ ecuaciones dado por $\mathbf{P Y}=\boldsymbol{P X}$, eliminó una ecuación porque entendía que también se cumplía que $\boldsymbol{P Y I}=\boldsymbol{P X I}$, con lo cual, en efecto, le sobraba una de las $\boldsymbol{n}$ ecuaciones del sistema $\mathbf{P Y}=\mathbf{P X}$. De haber procedido como se ha hecho en el presente texto, es decir, calcular primero los $\boldsymbol{n}$ precios absolutos y, a posteriori, calcular los relativos dividiendo ya los precios obtenidos por un precio cualquiera $\mathrm{O}$, por ejemplo, entre $\mathbf{P Y I}=$ constante, no hubiera tenido esa necesidad. En cualquier caso los resultados obtenidos para precios relativos de ambas maneras son los mismos 17 , con lo cual no podemos ser críticos en el proceder de Sraffa si el objetivo era buscar el resultado correcto. Sin embargo, al llegar al territorio de este capítulo, es decir al capítulo de la producción con excedente, sí hace un mal uso de las matemáticas por omisión del importante teorema de PerronFrobenius. El teorema es imprescindible para asegurar que un sistema como el dado en (2.5), es decir, para que $\boldsymbol{P Y}=\left(\mathbf{1}+\mathbf{g}_{\mathrm{m}}\right) \boldsymbol{P X}$ tenga una solución estrictamente ¡positiva! para los $\boldsymbol{n}$ precios; y ello sólo es posible si la matriz dada por el producto $\mathbf{X Y}^{-1}$ cumple los requisitos del teorema, es decir, que sea cuadrada, positiva e irreducible. Lo mismo ocurrirá con el cálculo de los multiplicadores de la mercancía-patrón que veremos más adelante en el capítulo correspondiente. Para completar 0 , mejor dicho, para aplicar el teorema a las ideas económicas que se exponen a partir de Sraffa es inevitable considerar que estamos en la producción simple, razón por la cual la matriz $\boldsymbol{Y}$ será una matriz positiva diagonal $\boldsymbol{n} \times \boldsymbol{n}$ de $\boldsymbol{n}$ elementos y su consecuencia es la de que su inversa $\boldsymbol{Y}^{-1}$ será positiva, puesto que la inversa de una matriz diagonal es otra matriz diagonal que se forma con los inversos de los elementos de la diagonal principal de la matriz original ${ }^{18}$.

En concreto y para buscar una solución al vector de precios y la tasa máxima de ganancia dada por la ecuación (2.5) $\mathbf{P Y}=\left(\mathbf{1}+\mathbf{g}_{\mathrm{m}}\right) \mathbf{P X}$ procedemos a post-multiplicar ésta por la inversa de $Y$ y, a su vez, a multiplicar ambos términos por $\left(1+g_{m}\right)^{-1}$ y queda:

\footnotetext{
16 Aunque Sraffa nunca hace la salvedad que eso es casi correcto (lo correcto son la condiciones del teorema Rouché-Frobenius mencionado) si ninguna ecuación es combinación lineal de las otras, pero tal pecadillo se lo podemos perdonar porque debemos suponer que Sraffa, en el contexto del modelo económico dado a partir de datos reales (los $\mathbf{Y}, \mathbf{X}$ e $\mathbf{L}$ pueden ser datos reales), es un suceso imposible que se dé una combinación lineal en la práctica. Creo que no es necesario más explicación en este aspecto.

17 Tenemos un sistema de $\mathbf{n}$ ecuaciones con $\mathbf{n}$ incógnitas, y suponemos que las $\mathbf{n}$ ecuaciones son linealmente independientes (es la forma vulgarizada del teorema de Rouché-Frobenius), con lo cual la solución es única sea cual sea el algoritmo de cálculo para llegar a la solución.

18 No merece la pena señalar que todos los elementos sin excepción deberán ser mayores o iguales a cero puesto que, en Economía, no tiene sentido tener menos algún bien. Aunque también es verdad que algún autor ha considerado, en el marco del análisis Input-Output de Leontief, que algunos bienes indeseables debieran ser incluidos en el modelo con signo negativo (por ejemplo, las armas).
} 
(2.10)

$\frac{1}{\left(1+g_{m}\right)} \times P=P X Y^{-1}$

Ahora en (2.10) es reconocible que la solución dada por el teorema de PerronFrobenius nos dará los $\boldsymbol{n}$ precios estrictamente positivos (el autovector de la matriz resultante $X Y^{-1}$ ) si tomamos el autovalor más alto en términos absolutos correspondiente, que es el único autovalor que garantiza el teorema que tanto $\mathbf{g}_{\mathrm{m}}$ como $\mathbf{P}$ van a ser estrictamente positivos. Para ello, el autovalor que viene dado por $\left(\mathbf{1}+\mathbf{g}_{\mathrm{m}}\right)^{-1}$, deberá ser menor o igual a uno ${ }^{19}$. Pero que el autovalor más alto sea menor que 1 eso no lo garantiza el teorema, eso viene dado si estamos en una economía productiva, es decir, si se cumple que $Y|>\boldsymbol{X}|$ o como mínimo mayor para algún bien. Sraffa siempre supondrá que se cumple lo anterior estrictamente, es decir, que siempre será mayor para todos los bienes el producto de cada bien que los bienes empleados como medio en el conjunto de la economía. Sin embargo puede muy bien ocurrir que estuviéramos en una economía donde, para algún bien, no hubiera excedente sino lo contrario y, a pesar de todo, se tuviera un vector de precios positivos para el sistema y también una tasa máxima de ganancia $\boldsymbol{g}_{\mathrm{m}}$ positiva. Daremos un ejemplo numérico calculado mediante la programación lineal. Es decir, el teorema no exige la productividad 20 del sistema puesto que este es un concepto de economía que se añade al modelo que exponemos a partir de Sraffa.

Ahora vamos a dar un ejemplo de las cuestiones anteriores, ejemplo extraído de la propia obra de Sraffa, pero del capítulo de la mercancía-patrón. Sea una economía dado por la matriz de productos, medios e inputs de trabajo siguientes:

\begin{tabular}{|l|l|lll|l|}
\cline { 2 - 4 } \multicolumn{1}{c|}{} & \multicolumn{3}{l|}{ Y= productos finales } & \multicolumn{1}{c|}{} \\
\cline { 2 - 4 } \multicolumn{1}{c|}{} & proceso 1 & proceso 2 & proceso 3 & sumas \\
\hline trigo & 180 & 0 & 0 & 180 \\
hierro & 0 & 270 & 0 & 270 \\
cerdos & 0 & 0 & 360 & 360 \\
\hline trigo & 90 & 30 & 30 & 150 \\
\hline
\end{tabular}

19 En el caso de que el autovalor fuera uno la tasa máxima de ganancia sería cero y estaríamos en el caso de que no hubiera excedente.

20 Este es uno de los pocos defectos que tiene el impagable y temprano libro de J. Vegara Economía política y modelo multisectoriales, 1979, que no separa claramente lo que se debe al teorema y lo que se debe a la productividad incorporada al sistema. 


\begin{tabular}{|l|lll|l|} 
hierro & 120 & 75 & 30 & 225 \\
cerdos & 60 & 90 & 150 & 300 \\
\hline
\end{tabular}

\begin{tabular}{|l|l|l|l|l|}
\cline { 2 - 4 } \multicolumn{4}{c|}{} & \multicolumn{3}{c|}{ inputs de trabajo } & \multicolumn{1}{l}{} \\
\cline { 2 - 5 } \multicolumn{1}{c|}{} & trigo & hierro & cerdos & total \\
\hline
\end{tabular}

Como puede comprobarse hay excedente para todos los bienes puesto que las sumas dadas en la última columna de la matriz $Y$ son mayores que las correspondientes de la matriz $\boldsymbol{X}$. Ahora procedemos, al igual que en el caso anterior, a calcúlar mediante programación lineal21 el vector de precios $\mathbf{P}$ y la tasa máxima de ganancia $\mathbf{g}_{\mathrm{m}}$ que satisface la ecuación matricial $\mathbf{P Y}=\left(\mathbf{1}+\boldsymbol{g}_{\mathrm{m}}\right) \boldsymbol{P X}$ (que actúa como restricción), a partir de la función objetivo PYI=1 (que actúa como numerario). La condición de restricción queda como:

\begin{tabular}{|c|c|c|c|c|}
\hline & trigo & hierro & cerdos & PYI \\
\hline$P Y=$ & 0,4783 & 0,2609 & 0,2609 & 1,0000 \\
\hline & & $a_{2}$ & $x$ & $x$ \\
\hline$\left(1+g_{m}\right) P X=$ & 0,4783 & 0,2609 & 0,2609 & 1,0000 \\
\hline
\end{tabular}

El resultado es un vector de precios jabsolutos! tal como:

\begin{tabular}{|l|l|l|l|}
\cline { 2 - 4 } & trigo & hierro & cerdos \\
\hline precios absolutos $=$ & 0,002657 & 0,000966 & 0,000725 \\
\hline
\end{tabular}

y una tasa máxima de ganancia $\mathbf{g}_{\mathrm{m}}$ que es del $\mathbf{2 0 \%}$. Hecho esto (equivalente al cálculo del autovalor más alto de $X Y^{-1}$ y su correspondiente autovector), procedemos a dividir (a posteriori) todos los precios entre el precio absoluto del trigo y obtenemos los precios relativos del resto en términos del precio del trigo 22:

${ }^{21}$ Cuyo método simplex lo podemos considerar como un algoritmo de búsqueda de la solución del teorema de Perrón-Frobenius, que lo es, a su vez, de los teoremas del punto fijo.

22 Podemos obrar así porque lo hacemos a posteriori, es decir, una vez calculados los precios absolutos. Si obráramos como Sraffa, es decir, añadiéramos una ecuación más al sistema tal como hacer algún precios igual a uno o dar un valor determinado a una macromagnitud agregada (tal como PYI O $\mathbf{P}(\mathbf{Y}-\mathbf{X}) \mathbf{I})$, calcularíamos los precios relativos directamente, con lo cual perderíamos una información relevante como son los precios absolutos (que representan en realidad los costes agregados en la producción de cada bien o servicio). Acabamos la nota diciendo que los precios relativos representan en Sraffa la relación de intercambio en términos físicos de las diversas mercancías (bienes y servicios) que permiten la reproducción del sistema en sus mismos términos. Los precios absolutos representarían, además, los costes de cada mercancía. 


\begin{tabular}{|l|l|l|l|}
\cline { 2 - 4 } \multicolumn{1}{c|}{} & trigo & hierro & cerdos \\
\hline precios relativos $=$ & 1,000 & 0,364 & 0,273 \\
\hline
\end{tabular}

Podría sorprender de que, a pesar de que hemos incluido el vector horizontal de inputs de trabajo $\boldsymbol{L}$, éste no influye en el cálculo de los precios. Matemáticamente es así porque en el modelo expuesto dado por la ecuación $\mathbf{P Y}=\left(\mathbf{1}+\boldsymbol{g}_{\mathrm{m}}\right) \boldsymbol{P X}$ no aparecen explícitamente estos inputs. Esto se entiende porque aún estamos en el cálculo del excedente, del cual es exponente la tasa máxima de ganancia ${ }^{23} \mathbf{g}_{\mathrm{m}}$. Cuando se proceda, análogamente a como hace Sraffa, a explicitar el reparto del excedente, aparecerán tanto los inputs de trabajo los salarios y la tasa de ganancia (sin más calificativo).

Como prometimos vamos a dar un ejemplo de cómo es posible obtener un vector de precios positivos y una tasa máxima de ganancia $\mathbf{g}_{\mathrm{m}}$ positiva a pesar de que, para algún bien o para varios, el excedente sea negativo, es decir, que para ese bien o esos bienes, el producto final del conjunto de la economía sea inferior a la suma de ese bien utilizado como medio en el conjunto de la economía. Veámoslo sin casi más explicaciones.

\begin{tabular}{|c|c|c|c|c|}
\hline & \multicolumn{3}{|c|}{$Y=$ productos finales } & \multirow[b]{2}{*}{ sumas } \\
\hline & proceso 1 & proceso 2 & proceso 3 & \\
\hline trigo & 180 & 0 & 0 & 180 \\
\hline hierro & 0 & 170 & 0 & 170 \\
\hline \multirow[t]{3}{*}{ cerdos } & 0 & 0 & 360 & 360 \\
\hline & \multicolumn{3}{|l|}{$\mathrm{X}=$ medios } & \\
\hline & proceso 1 & proceso 2 & proceso 3 & sumas \\
\hline trigo & 90 & 30 & 30 & 150 \\
\hline hierro & 120 & 75 & 30 & 225 \\
\hline cerdos & 60 & 90 & 150 & 300 \\
\hline
\end{tabular}

\begin{tabular}{|l|l|l|l|l|}
\cline { 2 - 5 } \multicolumn{1}{c|}{} & trigo & hierro & cerdos & total \\
\hline & 18,0 & 12,0 & 30,0 & 60,0 \\
\hline
\end{tabular}

Puede comprobarse ahora que, si bien sigue siendo excedentaria la producción de trigo y de cerdos, para el hierro es lo contrario y tenemos que

${ }^{23}$ Veremos que $\mathbf{g}_{\mathrm{m}}$ depende de $\mathbf{Y}$ y de $\mathbf{X}$, pero también de $\mathbf{L}$, es decir, de los inputs de trabajo. 
225 toneladas de hierro empleadas en el conjunto de la economía para producir los diversos bienes se contrastan con las 170 toneladas producidas. Es verdad que en este caso estaríamos en una economía a larga -incluso a medio plazo- no viable puesto que en pocas secuencias del flujo circular de la producción nos quedaríamos sin hierro. Pero eso no impide que, con el supuesto de déficit en este bien y en cada momento, pueda ${ }^{24}$ existir un conjunto de precios y de tasa máxima de ganancia que sean estrictamente positivos. Procedemos de la misma manera que antes con programación lineal (que busca un punto fijo para $\boldsymbol{P}$, lo cual le llevará a cumplir el teorema) y damos el resultado conjunto de precios absolutos y relativos como:

\begin{tabular}{|l|l|l|l|}
\cline { 2 - 4 } \multicolumn{1}{c|}{} & trigo & hierro & cerdos \\
\hline precios absolutos $=$ & 0,002743 & 0,001587 & 0,000657 \\
\hline precios relativos $=$ & 1,000 & 0,578 & 0,240 \\
\hline
\end{tabular}

Y la tasa máxima de ganancia $\mathbf{g}_{\mathrm{m}}$ obtenida es del $\mathbf{3 , 5 7 \%}$.

II - Podemos generalizar el modelo a múltiples tasas de ganancia $\mathbf{g}$ y de salarios $\boldsymbol{w}$. Sraffa nunca pasó de estas tasas unitarias a tasas más generales, pero esto puede hacerse sin que el modelo pierda capacidad explicativa. Por ejemplo, podría definirse o caracterizarse la economía y el excedente explícito de la misma mediante la ecuación matricial:

$$
P Y=P\left[X \otimes\left(I+G_{m}\right)\right]
$$

Y la expresión aritmética de la anterior

$$
\sum_{i=1}^{n} p_{i} y_{i j}=\sum_{i=1}^{n} p_{i}\left[x_{i j}\left(1+g_{i j}\right)\right] \quad \forall j=1 \quad a \quad n
$$

donde $\mathbf{G}_{\mathrm{m}}$ sería la matriz $\mathbf{n \times n}$ (podría ser diagonal, es decir, de solo $\boldsymbol{n}$ elementos ubicados en la diagonal principal, o de $\boldsymbol{n} \times \boldsymbol{n}$ elementos) de tasas máximas de ganancia. Aclarar que el producto interno de los medios $\boldsymbol{X}$ por la matriz resultante $\mathbf{I}+\boldsymbol{G}_{m}$ es un producto aritmético, es decir, se multiplica el medio producción $\mathbf{x}_{\mathbf{i j}}$ por la expresión $\left(\mathbf{1}+\mathbf{g m}_{\mathrm{ij}}\right)$ correspondiente para los $\boldsymbol{n} \times \boldsymbol{n}$ elementos ij. Pero esto no esta en Sraffa y forma parte de los posibles desarrollos de su gran texto. Lo que sí forma parte de su modelo es la explicitación del reparto del excedente (dado por $\mathbf{g}_{\mathrm{m}} \mathbf{P X}$ ) entre salarios $\boldsymbol{w}$ y ganancias $\mathbf{g}$ de acuerdo con su modelo:

${ }^{24}$ Que pueda existir no significa asegurar que sea segura su existencia. 


$$
\begin{aligned}
& p_{1} y_{1}=(1+g)\left[p_{1} x_{11}+p_{2} x_{21}+\ldots .+p_{n} x_{n 1}\right]+w l_{1} \\
& p_{2} y_{2}=(1+g)\left[p_{1} x_{12}+p_{2} x_{22}+\ldots .+p_{n} x_{n 2}\right]+w l_{2} \\
& p_{n} y_{n}=(1+g)\left[p_{1} x_{1 n}+p_{2} x_{2 n}+\ldots .+p_{n} x_{n n}\right]+w l_{n}
\end{aligned}
$$

Sraffa, por consideraciones históricas que vienen de los clásicos Smith, Ricardo, Torrens, Mill, Malthus, etc., no incluye los gastos salariales $\mathbf{w} \mathbf{l}_{\mathrm{j}}$ de cada bien dentro del corchete, es decir, exigiendo que la tasa de ganancia $\mathbf{g}$ se calcule sobre $\mathbf{w L}$ también. No discutimos aquí los pros y los contras de esta decisión, lo acertado o no que estuvo Sraffa al proceder así dado que intentamos incidir en los aspectos matemáticos que hay explícitos e implícitos en su modelo y no en una crítica intelectual del modelo. Sí es interesante desde el punto de vista formal que Sraffa ahora aumenta un grado de libertad el modelo al incluir dos variables nuevas como son salarios $\boldsymbol{w}$ y ganancias $\boldsymbol{g}$ y eliminar a su vez la tasa máxima de ganancia $\mathbf{g}_{\mathrm{m}}$. Ahora tendremos un sistema de $\boldsymbol{n}$ ecuaciones dado por la ecuación matricial $\mathbf{P Y}=\mathbf{w} \mathbf{L}+(\mathbf{1}+\mathbf{g}) \mathbf{P X}$ y $\mathbf{n}+\mathbf{2}$ variables dadas por los $\boldsymbol{n}$ precios absolutos ( $\boldsymbol{n}-\mathbf{1}$ precios relativos). Lo cual daría dos grados de libertad al sistema si partiéramos de los precios absolutos, o un grado de libertad si lo hacemos con los relativos 25 . Se puede elegir y Sraffa eligió este segundo criterio, aunque los resultados de los precios relativos -no así los absolutos-sean los mismos.

III - Sraffa introduce ya en este segundo capítulo de su obra la distinción entre bienes básicos y no básicos, pero no lo desarrolla. Ello lo hará en capítulos posteriores, por lo que allí nos veremos. Solo adelantar que ello está relacionado precisamente con la indescomponibilidad de la matriz $\mathbf{X Y}^{-1}$ que hemos visto para el aseguramiento de un vector de precios estrictamente positivo; y también está relacionado con la mercancía-patrón para el cálculo de un vector de multiplicadores a partir de la matriz $\boldsymbol{Y}^{-1} \boldsymbol{X}$ (no hay error en el orden de la multiplicación de la inversa de $Y$ ). Pero todo esto lo veremos más adelante.

Llegado a este punto Sraffa se detiene cuando tenía en sus manos lo que exponemos a continuación. Aunamos ahora las dos ecuaciones de definición del sistema hasta ahora empleadas como son la (2.5) y la (2.13):

25 Para evitar las complicación de Sraffa con el número de ecuaciones realmente independientes, lo que hacemos en este largo trabajo sobre PMpM es calcular siempre los precios absolutos (con lo cual no tenemos que eliminar ninguna de las $\mathbf{n}$ ecuaciones de las que partimos) y, a posteriori, es decir, una vez calculados los precios absolutos, se dividen los precios (y, por lo tanto, ino las ecuaciones!) entre un precio cualquiera o entre otra magnitud agregada cualquiera como pueda ser el producto nacional PYI o el excedente en términos de valor $\mathbf{P}[\mathbf{Y}-\mathbf{X}] \mathbf{I}$. 
(2.14)

$P Y=\left(1+g_{m}\right) P X$

$$
P Y=w L+(1+g) P X
$$

Si entre las dos anteriores eliminamos el escalar PXI queda:

$$
g=\left(1-\frac{w}{P(Y-X) I / L I}\right) g_{m}
$$

Y como regalo obtenemos - al eliminar esta vez los precios- la inquietante y sorprendente, pero magnífica:

$$
g_{m}=\frac{L X^{-1}(Y-X) I}{L I}
$$

donde comprobamos que la tasa máxima de ganancia $\mathbf{g}_{\mathrm{m}}$ ino depende de los precios ni de ninguna variable monetaria! Y si decía inquietante es porque nada garantiza que, para cualquier conjunto de productos finales $Y$, medios de producción $\boldsymbol{X}$ e inputs de trabajo $\boldsymbol{L}$, la tasa de ganancia máxima $\mathbf{g}_{\mathrm{m}}$ sea positiva dada la presencia de la inversa de $\boldsymbol{X}$ en el numerador en (2.17). En cuanto a (2.16) Sraffa obtiene una formulación parecida para solo la mercancía-patrón cuando también se puede obtener-como vemos- para el modelo de la economía real, pero con dos diferencias. En el caso de la mercancía-patrón, en lugar de la tasa de ganancia máxima $\mathbf{g}_{\mathrm{m}}$ tendremos la razón-patrón que Sraffa llama $\boldsymbol{R}$; en segundo lugar $y$, dado que Sraffa toma como numerarios $\mathbf{P}(\boldsymbol{Y}-\boldsymbol{X}) \mathbf{I}$ y $\mathbf{L I}$, se obtiene su famosa relación entre las variables distributivas:

$$
g=(1-w) R
$$

Es un misterio el porqué Sraffa relega esta relación a la mercancía-patrón cuando es válida para el modelo de economía real que viene dado por las ecuaciones (2.14) y (2.15), porque demostraremos en el capítulo correspondiente que esta razón-patrón $\boldsymbol{R}$ y la tasa máxima de ganancia $\mathbf{g}_{\mathrm{m}}$ son iguales en el modelo de producción simple, modelo en el que estamos de momento puesto que trabajamos con una matriz diagonal de productos finales. La cosa será distinta cuando entremos en territorio de la producción conjunta, donde $Y$ no es diagonal y su inversa tendrá elementos negativos. Además también demostraremos que no es necesario emplear ningún numerario para llegar a (2.18) con $\mathbf{g}_{\mathrm{m}}$ en lugar de $\boldsymbol{R}$. 
IV - Dos últimas reflexiones que nos merece este capítulo donde Sraffa introduce el excedente pero que no emplea el teorema de Perron-Frobenius. El teorema nos facilita que, a partir de un sistema de $\boldsymbol{n}$ ecuaciones, obtengamos n precios imás! un dato que es el autovalor que hace a los precios (autovector correspondiente) positivos, con lo cual con $\boldsymbol{n}$ ecuaciones obtenemos $\boldsymbol{n}+\mathbf{1}$ incógnitas. Ello solo es posible por las exigencias del teorema sobre la matriz original. Al no emplear Sraffa el teorema se obliga a emplear una condición más (una ecuación más) o a eliminar una ecuación de precios al menos. Por ello nunca pudo demostrar Sraffa que la tasa máxima de ganancia jsolo! dependa de los datos originales $26 \boldsymbol{L}, \boldsymbol{Y}$ y $\boldsymbol{X}$. De ahí que nunca pudo calcular los precios absolutos sino directamente los relativos, que son, en la práctica, variables no monetarias, relaciones de intercambio en términos físicos entre las diferentes mercancías. Con lo cual el modelo de Sraffa parecería más destinado a un modelo de economía de trueque en lugar de un modelo donde, en los precios reales, pueda incidir los aspectos monetarios 27 de la economía como la oferta monetaria o la cantidad de dinero.

La otra reflexión se refiere al deseo de escapar de Sraffa a cualquier consideración de rendimientos constantes que pudiera entenderse que se hace de forma explícita o implícita. La consideración la hace explícita Sraffa en el prólogo del libro por lo que no hay duda de sus intenciones. La pregunta es si, visto el desarrollo de su modelo, podemos sostener que no hace ningún supuesto sobre los rendimientos. Creo que la cuestión permanece abierta, pero algo tendremos que decir cuando lleguemos al capítulo sobre la reducción del capital a trabajo fechado. Pero también aquí podemos reflexionar sobre ello, porque si Sraffa hubiera construido su modelo con la condición de que los rendimientos fueran constantes, tal obligación podría venir formalizado mediante una ecuación tal que $\mathbf{L} / \mathbf{X I}$ fuera constante ${ }^{28}$ para todas las mercancías. Ello daría un protagonismo a los inputs de trabajo del que carecen hasta ahora. Así vemos que en la ecuación (2.18) no aparecen estos inputs, lo cual no parece aceptable. Tampoco hace Sraffa ninguna consideración sobre la necesidad de relacionar los salarios -la masa salarial en concreto dada por $w L I-c o n$ el producto nacional, con una relación tal como $\mathbf{w L I}=\mathbf{a P Y I}$, donde a sería un coeficiente que se movería entre cero y uno. Y con ello se cerraría su modelo y permitiría una relación de las variables distributivas entre sí y con el excedente más realista ${ }^{29}$. Pues bien, aunque estas cosas no están en Sraffa,

26 Sraffa creyó que con su ecuación de distribución $\mathbf{g}(\mathbf{1}-\mathbf{w}) \mathbf{R}$ había sorteado la influencia de los precios, pero realmente lo que hizo fue relativizar los precios, es decir, calcular directamente precios relativos y salarios relativos con (2.18). Ya veremos cómo se puede calcular una ecuación distributiva sin necesidad de ningún numerario.

27 Y ello a pesar de que Sraffa era un experto de temas monetarios ya que de estos temas fueron sus primeros trabajos cuando aún no había salido de Italia y luego más tarde, ya en Cambridge, cuando se mostró crítico con la teoría monetaria de su amigo y mentor J. M. Keynes.

28 La división entre los vectores $\mathbf{L}$ y $\mathbf{X I}$ sería aritmética y no matricial.

29 El aspecto negativo para el modelo de Sraffa que supondría incluir en él la ecuación wLI=aPYI es la de que se perdería un grado de libertad y con ello se correría el peligro de convertir en un modelo determinista el modelo de Sraffa, lo cual no sería aceptable. Este es un 
veremos los aspectos matemáticos de ampliación de estos supuestos en el modelo con el fin de enriquecerlos y acercarlo a la realidad sin que por ello pueda perder su capacidad explicativa, es decir, sin caer en un mero empirismo. Lo veremos más adelante, pero nos ceñiremos a los aspectos más formales puesto que el autor de este trabajo ha desarrollado todos estos temas en otros artículos ya publicados.

\section{Anexo a al capítulo II}

Los esrafianos -si queda alguno-tienen que reconocer que Sraffa no tiene una teoría de la distribución, al igual que ocurre con el marginalismo. Ambos tienen una teoría de la retribución de los factores, que es una cosa distinta aunque relacionada. Por ello nos vamos a permitir en este anexo -y por eso figura como anexo- completar el modelo de Sraffa con una ecuación sencilla, pero imprescindible, que relacione el valor de la producción global con la distribución de la renta. Sin esa ecuación, sin esa relación -como la tenían los fisiócratas- el modelo queda cojo, y queda cojo gravemente. Veamos esto y sus consecuencias. Supongamos que producción y renta estén relacionados de la manera más simple posible por la ecuación:

$$
w L I=a P Y I \quad \forall a \quad \text { tal que } \quad 0<a<1
$$

En lo anterior el coeficiente a estaría comprendido, en efecto, entre cero y uno y que, en la práctica, en las economías más desarrolladas y medido este coeficiente suele superar escasamente el $50 \%$. A esta ecuación fundamental que añadimos -y que representa la participación de los salarios $\boldsymbol{w}$ (o de la masa salarial wLI) en la producción PYI- le añadimos, a su vez, las ecuaciones de definición del sistema de Sraffa pero con salarios pre-factum:

$$
P Y=(1+g)[w L+P X]
$$

$$
P Y=\left(1+g_{m}\right) P X
$$

Además completamos las dos ecuaciones anteriores con la obtenida anteriormente de entre ambas tal como:

$$
g_{m}=\frac{L X^{-1}(Y-X) I}{L I}
$$

momento crítico de la obra de Sraffa y, quizás por ello, el economista turinés no incluyo esta relación en su modelo. No obstante esta reflexión se aparta sobremanera de la intención de este artículo, por lo que la cuestión queda ahí simplemente apuntada. 
Si eliminamos los precios entre (a.2) y (a.3), los reemplazamos en (a.1), tenemos en cuenta (a.4) y despejamos la tasa de ganancia g, queda:

$$
g=\frac{g_{m}-a\left(1+g_{m}\right)}{1+a\left(1+g_{m}\right)}
$$

Lo primera que comprobamos en la anterior ecuación es que han desaparecido los salarios y su función la ocupa el coeficiente a, es decir, la participación de las rentas salariales en el producto nacional; lo segundo que comprobamos es que la relación entre tasa de ganancia $\mathbf{g}$ y coeficiente a de participación es decrecientemente decreciente; tercero, que para que la tasa de ganancia $\mathbf{g}$ sea mayor que cero ha de ocurrir que el coeficiente de participación a ha de ser menor que $\mathbf{g}_{\mathrm{m}} /\left(\mathbf{1}+\boldsymbol{g}_{\mathrm{m}}\right) \mathrm{O}$, lo que es equivalente, que la tasa máxima de ganancia $\mathbf{g}_{\mathrm{m}}$ (recordemos, para que $\mathbf{g}>\mathbf{0}$ ) tiene que cumplir que $\mathbf{g}_{\mathrm{m}}>\mathbf{a} /(\mathbf{1}-\mathbf{a})$. El gráfico sería:

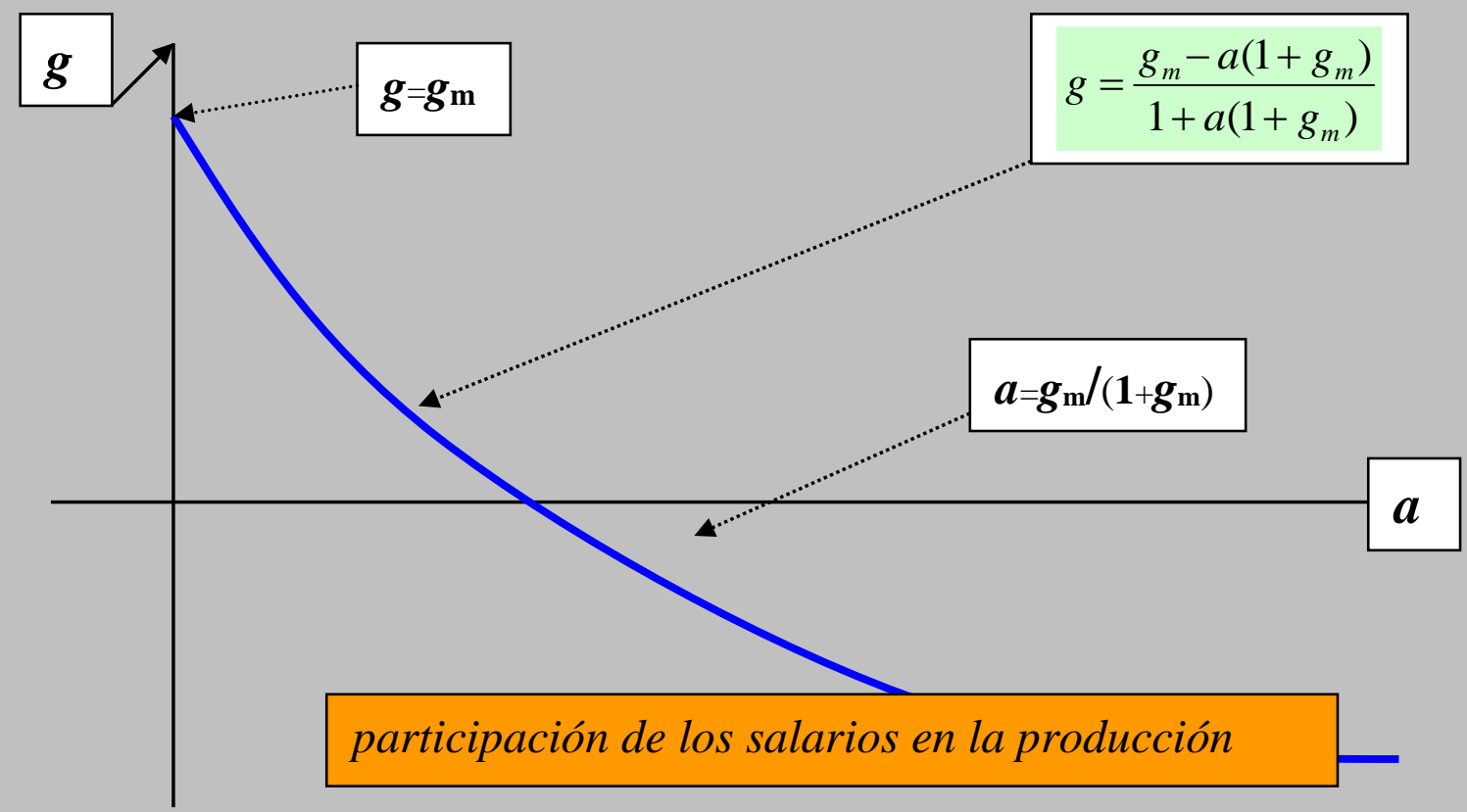

la ecuación (a.1) es la pérdida de libertad del sistema, porque ahora, una vez fijado la participación de los salarios en la producción, la tasa de ganancia queda determinada. Lo que ganamos en realismo lo perdemos en libertad, pero un desarrollo de la obra de Sraffa exigiría, entre otros supuestos, este como fundamental. 


\section{Anexo b al capítulo II}

En este capítulo hemos seguido a Sraffa en el empleo de tasas unitarias para las distributivas, pero eso puede entenderse, con razón, como una fuerte limitación del modelo esrafiano, de la misma manera que el uso de los salarios de los salarios post-factum en lugar de unos salarios que formen parte de los costes (pre-factum) puede entenderse, en este caso, como un error insoportable. Por ello, a lo largo de este trabajo vamos a trabajar indistintamente con ambos tipos de salarios. Sin embargo no hemos dado preferencia a trabajar con tasas generalizadas de salarios y ganancias en nuestro afán de seguir el libro de Sraffa y que este trabajo sirva de guía de estudio de la genial obra Producción de mercancías por medios de mercancías. Aquí, en este anexo, hacemos una excepción y vamos a completar el modelo de Sraffa con matrices generalizadas $\boldsymbol{n} \times \boldsymbol{n}$ para salarios $(\boldsymbol{W})$ y ganancias $(\boldsymbol{B})$, pudiendo tener también $\boldsymbol{n} \times \boldsymbol{n}$ elementos o solo $\boldsymbol{n}$ (matrices diagonales). Pues bien, una ecuación equivalente a la dada por:

$$
P Y=(1+g)[w L+P X]
$$

pero generalizada de acuerdo con lo anterior sería:

$$
P Y=L[W \otimes(I+B)]+P[X \otimes(I+B)]
$$

donde los productos de $\boldsymbol{W}$ y $\boldsymbol{X}$ con la matriz resultante $\mathbf{I}+\boldsymbol{B}$ lo son aritméticos, es decir, donde se multiplican el elemento genérico $\boldsymbol{w}_{\mathrm{ij}}$ de $\boldsymbol{W}$ por el correspondiente $\mathbf{1}+\boldsymbol{b}_{\mathrm{ij}}$ de $\mathbf{I}+\boldsymbol{B}$ y, de forma análoga, el genérico de $\boldsymbol{x}_{\mathrm{ij}}$ con el $\mathbf{1}+\boldsymbol{b}_{\mathrm{j}}$. El vector I sería un vector $\boldsymbol{n} \times \boldsymbol{n}$ de unos. Tenemos en (b.2) $\boldsymbol{n}$ ecuaciones pero con

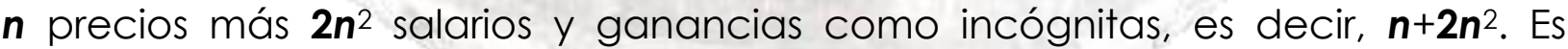
decir, tenemos $\mathbf{2} \mathbf{n}^{2}$ grados de libertad para los precios absolutos y $\mathbf{2 n}^{2} \mathbf{- 1}$ para los precios relativos. Ahora si despejamos los precios queda:

$$
P=L[W \otimes(I+B)] Y^{-1}+P[X \otimes(I+B)] Y^{-1}
$$

Apostamos y calculamos el vector de precios $\mathbf{P}$ como punto fijo al suponer que (b.3) es una función continua entre todas sus variables y que el conjunto de precios se mueve en un espacio cerrado, acotado y convexo. Podemos ir sustituyendo sucesivamente ( $\boldsymbol{t}$ veces) los precios de la ecuación (b.3) desde el 
primer miembro de la ecuación en el vector de precios que figura en el segundo sumando del lado derecho de la ecuación y queda:

$$
P=\left[\sum_{k=0}^{t-1} L[W \otimes(I+B)] Y^{-1}\right]\left[[X \otimes(I+B)] Y^{-1}\right]^{k}+P\left[[X \otimes(I+B)] Y^{-1}\right]^{t}
$$

Y si agrupamos los precios en el primer término queda:

$$
P=\left[\left[\sum_{k=0}^{t-1} L[W \otimes(I+B)] Y^{-1}\right]\left[[X \otimes(I+B)] Y^{-1}\right]^{k}\right]\left[I-\left[[X \otimes(I+B)] Y^{-1}\right]^{t}\right]^{-1}
$$

Y esta ecuación bien podría describir la formación de los precios en la economía de un país. Es verdad que faltan de forma explícita los medios de producción de capital fijo, pero podemos considerar estos formando tanto de los medios de producción como de los productos finales, no como bienes finales sino como bienes valorados (es decir, con precios no de mercado) año a año. También podemos considerar que tanto las exportaciones como las importaciones están incluidas en medios y productos valorados en moneda nacional, porque nada se dice en la ecuación ni de donde viene $\boldsymbol{X}$ como medios, ni quienes consumen $\boldsymbol{Y}$ como productos. También está el sector público como productor y consumidor, porque nada se dice del espacio jurídico (público o privado) en el que se mueve producción y consumo. Consideramos también que en $\boldsymbol{Y}$ están los medios de producción que en el período siguiente van a formarte parte de los medios $\boldsymbol{X}$ y también están en $\boldsymbol{Y}$ los bienes de consumo ${ }^{30}$. Lo que falta es el sector público como responsable del sistema tributario. La ecuación (b.5) no es precisamente una ecuación determinista puesto que tiene, como hemos visto, $\mathbf{2 n}^{2}-1$ grados de libertad puesto que no hemos introducido ninguna variable nueva ni ninguna ecuación nueva respecto (b.3). Es, por tanto, esta ecuación compatible con cualquiera de las formas de mercado en las que se mueve la realidad y compatible también con las formas de mercado teorizadas por la microeconomía convencional. Dicho esto, si el modelo de Sraffa es incompatible-que creo que lo es- con la microeconomía convencional es por otros motivos como son la consideración de los precios como un margen sobre los costes, el desarrollo de su modelo basado en las relaciones intersectoriales, su visión del capital como trabajo fechado, sus consideraciones sobre el capital fijo o sus avances en la producción conjunta, aspectos que la microeconomía convencional no contempla.

30 Podríamos hacer explícitos estos bienes con la ecuación $P_{c} C+P Y=L[W \otimes(I+B)]+P[X \otimes(I+B)]$, donde $\mathbf{C}$ sería la matriz de bienes de consumo (no necesariamente cuadrada) y $\mathbf{P}_{\mathrm{c}}$ su vector de precios correspondiente. 
Vamos a completar estas generalizaciones introduciendo la ecuación de Sraffa sobre las tasas máximas de ganancias pero también generalizadas $\boldsymbol{B}_{m}$ con las mismas consideraciones anteriores sobre la multiplicación aritmética y no matricial de la matriz $\boldsymbol{X}$ sobre la nueva matriz resultante $\mathbf{I}+\boldsymbol{B}_{\mathrm{m}}$. Esta ecuación sería:

$$
P Y=P\left[X \otimes\left(I+B_{m}\right)\right]
$$

Entre esta ecuación y la (b.2), eliminando PY, sale:

$$
P=L[W \otimes(I+B)]\left[X \otimes\left(B_{m}-B\right)\right]^{-1}
$$

Si ahora sustituimos los precios obtenidos en (b.7) en (b.3), obtenemos definitivamente:

$$
P=L[W \otimes(I+B)]\left[I+\left[X \otimes\left(B_{m}-B\right)\right]^{-1}[X \otimes(I+B)]\right] Y^{-1}
$$

Y la anterior es una alternativa a (b.5) porque en (b.8) aparece la matriz $\boldsymbol{B}_{\mathrm{m}}$ de ganancias máximas, matriz que (b.5) no contempla. 
Anexo c al capítulo II

No hay dificultad para desarrollar y demostrar gran parte de las ideas y proposiciones de Sraffa a partir de estas matrices de salarios $\boldsymbol{W}$ y ganancias $\boldsymbol{B}$ y ganancias máximas $\boldsymbol{B}_{\mathrm{m}}$ (también se pude completar con salarios máximos que llamaríamos $\boldsymbol{W}_{\mathrm{m}}$ ). Sin embargo, al embarcarse en semejante viaje, perdemos el asidero del teorema de Perrón-Frobenius. Además, lo normal es que trabajáramos con más grados de libertad puesto que, en lugar de tener $\mathbf{2}$ variables distributivas, vamos a tener $\mathbf{2} n^{2}$ variables o $\mathbf{4} n^{2}$ si añadimos las matrices $\boldsymbol{n} \times \boldsymbol{n}$ de ganancias máximas y salarios máximos. Es verdad que tendremos que acotar siempre que ganancias y salarios han de ser menores o a lo sumo iguales que ganancias y salarios máximos; también es posible que haya que acotar las variables a otras circunstancias aconsejables como, por ejemplo, relacionar la producción global PYI con los diversos tipos de rentas. Entonces se plantea si podemos relacionar y representar un modelo de salarios y ganancias llamemos generalizadas mediante el modelo esrafiano de variables distributivas unitarias. Por supuesto que Sraffa no se planteó tal problema porque tampoco se planteó la generalización. El problema entonces en términos formales se reduce a considerar si una ecuación como la:

$$
P Y=L[W \otimes(I+B)]+P[X \otimes(I+B)]
$$

pudiera ser reducida a una ecuación esrafiana (pero con salarios pre-factum) como:

$$
P Y=(1+g)[w L+P X]
$$

pero que nos diera jel mismo vector de precios! que (c.1). Un procedimiento pudiera ser igualar ambas ecuaciones a trozos tal como:

$$
(1+g) P X=P[X \otimes(I+B)]
$$

$$
(1+g) w L=L[W \otimes(I+B)]
$$

$Y$, entonces, a partir de ambas ecuaciones, obtener el valor de las variables unitarias $\mathbf{g}$ y $\mathbf{w}$ además del vector de precios $\boldsymbol{P}$ que satisfacen todas las ecuaciones anteriores. La ecuación (c.3) podemos escribirla como:

$$
g P X=P[X \otimes B]
$$


al eliminar el vector resultante común en ambos miembros de la igualdad $\mathbf{P X}$. Ahora post-multiplicamos por la inversa de $\boldsymbol{X}$ ambos miembros de la igualdad y queda:

$$
g P=P[X \otimes B)] X^{-1}
$$

Si la matriz resultante $[\boldsymbol{X} \otimes \boldsymbol{B}] \boldsymbol{X}^{-1}$ fuera positiva e irreducible (es cuadrada por construcción), podríamos aplicar Perrón-Frobenius y obtener el vector de precios $\boldsymbol{P}$ como autovalor de la matriz resultante anterior. Además, la tasa de ganancia $\mathbf{g}$ sería el autovalor correspondiente tal que $\boldsymbol{P}$ fuera estrictamente positivo. No hay garantía de esta matriz resultante cumpla todos los requisitos del teorema, pero con algunos valores convenientes de $\boldsymbol{Y}, \boldsymbol{X}$ y $\boldsymbol{L}$ podría ocurrir. La dificultad viene ahora con la ecuación (c.4). Hagamos algo similar a lo anterior y consideremos si esta ecuación se puede aplicar Perrón-Frobenius. La respuesta es no porque, para ello, debería ocurrir que los elementos del vector de inputs de trabajo $L$ fueran variables a determinar y no jdatos de partida! de este supuesto modelo generalizado a partir de Sraffa. La conclusión es la de que no es posible -o al menos no se ve cómo salvar las dificultades-para poder representar una economía como la definida a través de (c.1) por medio de (c.2). Es verdad que si consideramos que, o bien todos los mercados son de competencia perfecta y entonces existe una única tasa de ganancia (pero también hay que considerar una sola tasa de salarios), que es la teoría de la unificación de las ganancias a través de la competencia; o bien consideramos que, a largo plazo, todas las diversas tasas de ganancia convergen a un valor que podamos llamar (peligrosamente) natural, normal o similar, que es la teoría de la gravitación. A. Roncaglia exploró ambas posibilidades en su estudio sobre los precios ${ }^{31}$. No entramos a discutir esos aspectos por la naturaleza de este trabajo ceñido a los aspectos matemáticos de la obra de Sraffa, pero ahí queda apuntada la cuestión conceptual y, también, la cuestión formal y la imposibilidad de representar con el modelo de Sraffa una generalización (conveniente) del mismo.

\section{Capítulo III - TRABAJO Y MEDIOS DE PRODUCCIÓN}

I - Los economistas clásicos indagaron o especularon -según cómo se mire- en la economía las relaciones entre el capital y el trabajo que no estuvieran distorsionadas por la variación de los precios. Eso le llevó a Marx a sus

${ }^{31}$ Roncaglia, Alessandro (1978): "Sraffa and the Theory of Prices", New York: Wiley, [Sraffa e la teoria dei prezzi, 1975]. 
consideraciones sobre lo que el llamaba composición orgánica de capital a partir de su teoría del valor-trabajo importada con matices de David Ricardo. Pero Sraffa sigue otro camino porque le parecía que achacar a los precios consideraciones sobre el valor intrínseco de los bienes y servicios -dicho en terminología moderna- le parecía mera metafísica ${ }^{32}$. Pero no por ello desestimó su importancia y sus esfuerzos intelectuales le llevó al descubrimiento, quizás no enteramente original, de la mercancía-patrón. Para facilitar las consideraciones de Sraffa en el capítulo III vamos a desarrollar y despejar los precios en la ecuación de definición de su modelo que hemos expuesto anterioremente. En efecto, si despejamos los precios obtenemos:

$$
P=w L[Y-(1+g) X]^{-1}
$$

Y si a (20) le añadimos la relación entre medios y productos dada por la ecuación matricial $\boldsymbol{X}=\boldsymbol{A} \boldsymbol{Y}$ obtenemos de (3.1):

$$
P=w L Y^{-1}[I-(1+g) A]^{-1}
$$

Y si en lugar de la ecuación (3.2) hubiéramos partido de la más realista ecuación:

$$
P Y=(1+g)[w L+P X]
$$

hubiéramos obtenido la más realista 33 también:

$$
P=(1+g) w L Y^{-1}[I-(1+g) A]^{-1}
$$

Si desarrollamos la (3.4), por ejemplo, desarrollando la matriz que aparece en el segundo término, es decir, desarrollando $[\mathbf{I}(\mathbf{1}+\mathbf{g}) \mathbf{A}]^{-1}$ queda:

$32 \mathrm{H}$. Kurz recoge en un trabajo del 2007 sobre las notas inéditas de Sraffa unas palabras suyas referidas a la teoría del valor diciendo que "toda investigación acerca del valor ha sido siempre (y aún es y probablemente siempre lo será) una búsqueda puramente metafísica" (Las contribuciones de Sraffa a la Economía: algunas notas sobre sus trabajos inéditos).

33 Aunque las tasas unitarias aún nos alejen de un mayor realismo, pero eso es subsanable trabajando con matrices de ganancias y salarios como se hace en determinados momentos en este trabajo. También la generalización tiene su coste intelectual, porque eso obliga a desechar la teoría del uso de tasas unitarias como efecto de la competencia llevada hasta sus útlimas consecuencias. En todo caso queda la teoría de la gravitación para la formación de los precios porque esta es sostenible con ganancias positivas y desiguales. 


$$
P=(1+g) w L Y^{-1}\left[I+(1+g) A+(1+g)^{2} A^{2}+\ldots+(1+g)^{n-1} A^{n-1}\right]
$$

Vemos que las posibles variaciones entre el capital y el trabajo (representados por la matriz $\boldsymbol{A}$ de relaciones medios y productos), el trabajo representado por los inputs de trabajo $\boldsymbol{L}$ y la tasa salarial $\boldsymbol{w}$, pueden ser algo más complejas que las que expone Sraffa. La razón de ello es que aparece un tercer elemento que es el tiempo, aspecto que se refleja en los exponentes que llevan las expresiones $\mathbf{1 + g}$ y la matriz $\mathbf{A}$, donde cada elemento $\boldsymbol{a}_{\mathrm{ij}}$ representa la cantidad del bien $\boldsymbol{i}$ del proceso o sector $\boldsymbol{j}$ que es necesario para producir una unidad del bien $\boldsymbol{j}$. De (3.7) sí obtenemos una expresión significativa si no hubiera ganancias, es decir, si hacemos $\mathbf{g}=\mathbf{0}$ nos da:

$$
P=w L Y^{-1}\left[I+A+A^{2}+\ldots+A^{n-1}\right]
$$

Y los precios jserían proporcionales a los medios empleados 34 !, pero no directamente, sino a la suma de los medios y trabajo empleados directamente como es el primer término de (3.6) $L Y^{-1}$ y a los medios empleados indirectamente a lo largo del tiempo, que representan sucesivamente $L Y^{-1} \mathbf{A}, L Y^{-}$ ${ }^{1} A^{2}, \ldots, L Y^{-1} A^{n-1}$. La constancia de la matriz $A$ a lo largo del tiempo nos vuelve a las consideraciones de Sraffa que hace en el prólogo sobre la constancia o no de los rendimientos del capital. Viendo (3.5) y (3.6) debemos inclinarnos a que, al menos en este capítulo, el modelo de Sraffa, más allá de sus intenciones, ha de suponerse de rendimientos constantes. Otra cosa sería una ecuación tal como:

$$
P=(1+g) w L Y^{-1}\left[I+(1+g) A_{1}+(1+g)^{2} A_{2}^{2}+\ldots+(1+g)^{n-1} A_{n-1}^{n-1}\right]
$$

donde las matrices $\boldsymbol{A}$ que, recordemos, relacionan medios de producción $\boldsymbol{X}$ y productos finales $\boldsymbol{Y}$ (recordar que $\boldsymbol{X}=\boldsymbol{A} \boldsymbol{Y}$, es decir, $\boldsymbol{A}=\boldsymbol{X} \boldsymbol{Y}^{-1}$ ), cambian a lo largo del tiempo. Pero volviendo a la ecuación (3.6), podemos afirmar que los precios, en el caso de que las ganancias sean cero, son proporcionales a los inputs de trabajo $L$, pero que también dependen de forma que, podemos entender, ponderadas 35 por las relaciones entre medios y productos dadas por las sucesivas e hipotéticas $\boldsymbol{A}, \boldsymbol{A}^{1}, \boldsymbol{A}^{2}$, etc. Creo que estos desarrollos explícitos del modelo de Sraffa le hubieran hecho feliz y, quizás más aún, a David Ricardo. También a Marx, aunque le hubieran puesto al teutón ante la tesitura de cuestionar su propia teoría del valor trabajo, el llamado problema de la

\footnotetext{
34 Y el deseo de D. Ricardo se hubiera cumplido porque en (25) se establece una relación proporcional entre precios y trabajo aunque mediada por las relaciones capital/producto que representan los elementos de la matriz $\mathbf{A}$.

35 No hay dificultad matemática por convertir las sucesivas $\mathbf{A}, \mathbf{A}^{2}$, ponderada de alguna matriz media hipotética obtenida a partir de $\mathbf{A}$.

$\mathbf{A}^{\mathrm{n}-1}$ en una media
} 
transformación de estos a precios, pero que Marx erró36 en el libro tercero de El Capital.

II - El esfuerzo de Sraffa es encomiable y desconocemos, al menos por ahora, si desarrolló por su cuenta o con ayuda de los matemáticos que hemos mencionado cómo son estas relaciones si relacionamos por cociente un precio cualesquiera dado por (3.5) entre otros precios. El que sí lo hace es Pasinetti en su libro Lecciones de Teoría de la Producción (epígrafe 5 del capítulo 5), que titula Estructura de precios correspondiente a distintos niveles del tipo de beneficio ${ }^{37}$. Pasinetti analiza como cambian los precios cuando se mueve la tasa de ganancia. Ahora cabe preguntarse lo contrario: żqué pasa si se hacen cero los salarios? Es evidente que entonces no podemos partir de la ecuación (3.5), pero sí lo podemos hacer a través de (3.8), es decir:

$$
P Y=\left(1+g_{m}\right) P X
$$

donde el excedente $\boldsymbol{g}_{\mathrm{m}} \boldsymbol{P X}$ proporcionado por cada bien se los lleva la empresa (y el total vendría dado por $\mathbf{g}_{\mathrm{m}} \mathbf{P X I}$ ). En este caso debe suponerse que existen salarios implícitos en los medios de producción, es decir, que el trabajo entra en pie de igualdad "con el petróleo para las máquinas o los alimentos para el ganado" (Sraffa, capítulo II, epígrafe 8). Sraffa, en todo caso, se aproxima bastante al resultado cuando señala que "los movimientos relativos de dos productos vienen a depender, no solo de las proporciones entre trabajo y medios de producción con los que se han obtenido, sino también de las proporciones mediante las que estos medios de producción y aquellos medios de producción han sido obtenidos, y así sucesivamente"38. Viendo la ecuación (20), a Sraffa le faltó añadir que el tiempo y los inputs de trabajo juegan también un papel en estas relaciones entre precios, pero el texto mencionado resume al menos muy bien todo el capítulo III de PMpM.

III - Vamos a obtener una ecuación de precios que nunca dedujo Sraffa en su modelo y que, sin embargo, no tenía para ello que hacer ningún supuesto adicional a los considerados en los dos capítulos vistos hasta ahora. Traemos a colación las ecuaciones de definición del sistema dado por:

$$
P Y=\left(1+g_{m}\right) P X
$$

36 Y que Marx era perfectamente consciente del error, pero lo aceptó como una primera aproximación en aras de convertir sus valores en precios. Y no podía acertar porque se necesita el teorema de Perrón-Frobenius para una solución correcta y estos teoremas se descubrieron a comienzos del siglo XX cuando Marx ya no estaba en el mundo de los vivos. También se puede aproximar a la solución correcta con programación lineal o utilizando cadenas de Markov.

37 Pag. 109 en la edición española de 1983.

${ }^{38}$ Capítulo Il, epígrafe 20 de PMpM. 


$$
P Y=w L+(1+g) P X
$$

Pues bien, de entre ambas ecuaciones obtenemos que los precios valen:

$$
P=\frac{w}{g_{m}-g} \times L X^{-1}
$$

Y si en lugar de la (15), que es la de Sraffa, utilizamos la más realista de salarios pre-factum -en lugar de los post-factum-expresada en (3.3) sale:

$$
P=\frac{(1+g) w}{g_{m}-g} \times L X^{-1}
$$

Viendo tanto (3.11) como (3.12) parecería que los productos finales no inciden en los precios, pero no ocurre tal porque recordamos que la tasa máxima de ganancia $\mathbf{g}_{\mathrm{m}}$ viene expresada por:

$$
g_{m}=\frac{L X^{-1}(Y-X) I}{L I}
$$

donde sí están los productos finales $Y$. Hemos mencionado anteriormente que Sraffa no se dio cuenta en su plenitud ${ }^{39}$ de que tenía ante sí una teoría de la inflación que podríamos llamar, actualmente, estructural. En efecto, si los empresarios, fruto de las condiciones de mercado y de sus ambiciones por ganar dinero, pudieran llevar sus ganancias cercanas a las tasas máximas -que es equivalente a la apropiación de todo el excedente-, tanto en (3.11) como en (3.12) los precios tenderían al infinito. Y decimos que la inflación sería estructural porque no dependería de la política monetaria, es decir, no dependería del comportamiento de los mercados monetarios, no dependería de las relaciones de oferta y demanda de dinero, ni dependería de las políticas de los bancos centrales en aras de controlar la cantidad de dinero en circulación con el fin de controlar, a su vez, la inflación, que deberíamos llamarla entonces inflación monetarista. Sraffa, tan incisivo y conspicuo en otros

39 Ver el apéndice B de $\mathbf{P M p M}$. Ahí Sraffa llega a publicar un gráfico que relaciona precios y tasa de ganancia, donde se ve esta relación y la subida al infinito si las ganancias se aproximan a las ganancias máximas, pero él estaba interesado en este apéndice en los efectos sobre las ganancias cuando establece distingos entre bienes básicos y no básicos. Quizás por ello, discutiendo una cuestión menor, se le pasó por alto lo que podría haber sido un capítulo más que interesante de su obra. 
aspectos, aquí se le escapó una ocasión de oro para lanzar algún dardo - y no solo al marginalismo imperante en su época- a lo que luego se llamaría monetarismo (a partir de las obras 40 de Milton Friedman). Acabamos con esto y esperamos que al menos todo lo anterior sirva para facilitar la lectura y las conclusiones de uno de los capítulos más interesantes del libro de Sraffa, capítulo precisamente difícil de seguir y entender -sí las intenciones y supuestos- porque precisamente Sraffa no hace explícito ningún formalismo, ninguna ecuación que ayude a su comprensión. Eso sí, nos dejó reflexiones, parcas de estilo como era el suyo, pero siempre originales, profundas e interesantes. Sraffa fue sin duda uno de los grandes intelectuales de su siglo y no solo un mero economista por grande que füera. Lástima que su obra haya sido académicamente relegada al olvido.

Anexo a al capítulo III

En el epígrafe 15 del capítulo III de PMpP dice Sraffa que "La claye del movimiento de precios relativos que sigue a una variación en el salarios consiste en la desigualdad de las proporciones en que el trabajo y los medios de producción son empleados en las distintas industrias". ¿̇Tiene razón Sraffa? Previamente supone el economista italiano que "toda la renta nacional va al trabajo". Vamos a abordar matemáticamente el tema con carácter general y podremos, en todo caso, particularizar para el caso de que las tasas de ganancia fueran cero si ello es pertinente. Vamos a partir de las ecuaciones que conocemos y que definen la economía de Sraffa pero con salarios prefactum:

$$
P Y=(1+g)[w L+P X]
$$

40 Hay que recordar que Sraffa muere en 1983 y que Friedman publica en 1962 Capitalism and Freedom, pero, además, ya había escrito el norteamericano muchos artículos sobre temas monetarios en fechas más tempranas, es decir, en fechas anteriores a la publicación de Sraffa de Producción de mercancías por medios de mercancías, que lo fue en 1960. 


$$
P Y=\left(1+g_{m}\right) P X
$$

Aunque Sraffa dice con ironía en el prólogo de PMpM que, aunque él no hace un supuesto "tácito de rendimientos constantes", si alguien "encuentra útil tal supuesto no hay inconveniente alguno". En el epígrafe 17, en cambio, nos habla de "una proporción crítica entre el trabajo y los medios de producción". Veremos también que en la reducción del capital (medios de producción) a trabajo fechado supone también implícitamente permanencia entre trabajo y medios. Pues bien, estos textos y otros muchos de PMpM, que una veces son contradictorios y otros lo son solo en apariencia, nos autorizan a su vez a establecer como esrafiana una relación entre trabajo y medios -que casi nunca hace explícita- tal como:

$$
l_{i}=k_{i} \sum_{j=1}^{n} x_{i j} \quad \forall i=1 \quad a \quad n
$$

Esta última ecuación representa, de forma genérica, la relación entre el trabajo empleado en cada bien o servicio y el total de este mismo medio de producción empleado en el conjunto de los procesos ( $\mathbf{j}=\mathbf{1}$ a $\mathbf{n}$ ). En términos matriciales sería:

$$
L_{n x 1}^{T}=\underset{n x 1}{K \otimes}\left(\underset{n x n n x 1}{X} I_{n}\right)
$$

Con el signo de multiplicación ${ }^{41}$ que hay entre el vector $\boldsymbol{K}$ y el vector resultante XI queremos indicar que se trata de un producto aritmético de los dos vectores y no un producto matricial. Es decir, en la ecuación anterior se multiplica el elemento genérico $\boldsymbol{k}_{\mathrm{i}}$ por el elemento también $\boldsymbol{i}$ de la suma de todos los medios $\mathbf{x}_{\mathrm{ij}}$ que corresponden a un mismo producto, razón por la cual sumamos todos los procesos $\boldsymbol{j}$ implicados en un mismo bien o servicio $\boldsymbol{i}$. De momento dejamos la ecuación (a.4) y seguimos con las ecuaciones matriciales (a.1) y (a.2) para obtener la ecuación de precios:

$$
P=\frac{(1+g) w}{g_{m}-g} \times L X^{-1}
$$

41 Estamos forzados a emplear la transpuesta de $\mathbf{L}$, es decir, $L^{\top}$ dada la relación que se ha establecido entre trabajo y medios de producción en (a.3). 
Si ahora sustituimos los precios de (a.5) en el vector de precios que premultiplica a los medios $\boldsymbol{X}$ en la ecuación (a.1) y despejamos el vector de precios que multiplica a la matriz $\boldsymbol{Y}$ queda:

$$
P=(1+g)\left[w L Y^{-1}+\left(\frac{(1+g) w}{g_{m}-g} \times L X^{-1}\right) X\right] Y^{-1}
$$

que tras manipulaciones elementales:

$$
P=\frac{(1+g)\left(1+g_{m}\right) w}{g_{m}-g} L Y^{-1}
$$

En (a.7) ya vemos que un precio cualquiera i podría estará representado por:

$$
p_{i}=\frac{(1+g)\left(1+g_{m}\right) w}{g_{m}-g} \times \frac{l_{i}}{y_{i i}} \quad \forall i=1 \quad a n
$$

Hemos supuesto que estamos en la producción simple y, por tanto, la matriz $Y$ es una matriz diagonal, con lo que la matriz inversa de $Y$ estará formada por los inversos de los elementos de la diagonal de $Y$. En el caso de la producción conjunta no habría mayor problema, pero entonces una ecuación como (a.9) representaría los precios:

$$
p_{i}=\frac{(1+g)\left(1+g_{m}\right) w}{g_{m}-g} \times \sum_{j=1}^{n} l_{i} z_{i j} \quad \forall i=1 \quad a \quad n
$$

donde $\mathbf{z}_{\mathrm{ij}}$ sería el elemento inverso correspondiente al lugar que surge de $\boldsymbol{Y}^{-1}$. Otra cosa es buscarle el sentido económico al sumatorio de (a.9). De (a.8) podemos obtener la relación entre un precio cualquier $\boldsymbol{s}$ y otro que llamaremos $\boldsymbol{t}$. Pues bien, dado que todos los términos que anteceden a $\boldsymbol{l}_{\mathrm{i}} / \boldsymbol{y}_{\mathrm{ii}}$ son iguales para los dos precios, un cociente de precios para estas dos mercancías $\mathbf{s}$ y $\boldsymbol{t}$ sería:

(a.10)

$$
\frac{p_{s}}{p_{t}}=\frac{l_{s}}{y_{s s}} / \frac{l_{t}}{y_{t t}}
$$

En el caso de la producción conjunta quedaría: 


$$
\frac{p_{s}}{p_{t}}=\frac{l_{s} \sum_{i=1}^{n} z_{i s}}{l_{t} \sum_{i=1}^{n} z_{i t}}
$$

De lo anterior podemos concluir que la relación de precios de dos bienes o servicios (mercancías en Sraffa) no dependen de las variables distributivas, sino tan solo de los inputs de trabajo concernidos y de las estructuras de capital/producto que representan, en este caso, las $\boldsymbol{z}_{\text {is }}$ y $\boldsymbol{z}_{\text {it. }}$. En Sraffa, fruto de su modelo y no de posibles elucubraciones, los precios son meras relaciones de intercambio entre mercancías aunque para las empresas sean también reflejo de sus costes. Si consideráramos lo que dice Sraffa en el texto que hemos leído, pudiera estar representado su modelo por la ecuación matricial $\mathbf{L}=\mathbf{K X}$, donde $\mathbf{L}$ es un vector que va de $\mathbf{1}$ a $\boldsymbol{n}$ y $\boldsymbol{K}$ otro vector con el mismo recorrido. Si a lo anterior le añadimos la ecuación de coeficientes $\boldsymbol{X}=\boldsymbol{A} \boldsymbol{Y}$, donde $\boldsymbol{Y}$ es diagonal, sale:

$$
P=\frac{(1+g)\left(1+g_{m}\right) w}{g_{m}-g} \times K A
$$

Y para el cociente de dos precios cualesquiera que antes hemos llamado $\boldsymbol{p}_{\mathrm{s}} \mathrm{y}$ p+quedaría:

$$
\frac{p_{s}}{p_{t}}=\frac{\sum_{j=1}^{n} k_{s} a_{s j}}{\sum_{j=1}^{n} k_{t} a_{t j}}
$$

Vemos que la relación que obtenemos de los precios es entre los medios de producción y los productos finales respectivos, escapándonos de nuevo la relación entre trabajo y medios. No es que esta no exista-puesto que la hemos introducido como hipótesis- pero el resultado para los precios es que el cociente de dos de ellos cualesquiera ha de medirse entre el input de trabajo y el producto final respectivo, o bien, entre el medio de producción y el producto final. En todo caso si establecemos una relación entre los inputs de trabajo y los medios por un lado (como hipótesis $\boldsymbol{L}=\boldsymbol{K} \boldsymbol{X}$ ) y, además, partimos también de una relación entre medios y productos dada por $\boldsymbol{X}=\boldsymbol{A} \boldsymbol{Y}$, se concluye que también existirá una relación entre inputs de trabajo y medios, aunque no la hagamos explícita. Podemos concluir que Sraffa tiene razón al establecer que la relación de precios no se ve afectada cuando partimos de la hipótesis de que el input de trabajo de una mercancía cualquiera está relacionada con la suma de 
todos los procesos que producen esa mercancía ${ }^{42}$ (si fuera por proceso la relación no tendría ningún valor puesto que eso sería una definición). Además no es necesario suponer para esta conclusión que "toda la renta nacional va al trabajo", como hace Sraffa. Por supuesto que también ocurre bajo ese supuesto, pero las ganancias y salarios se eliminan al buscar esa relación, con lo cual $(a .10),(a, 11)$ y (a.13) son ciertas, sean cuales sean los salarios y ganancias; y ello es debido a que hemos trabajado con tasas unitarias. Con tasas múltiples es fácil colegir -incluso sin matemáticas- que la relación de precios va a depender, además de la relación entre inputs de trabajo y medios (o alternativamente, como hemos visto, entre medios y productos), y también entre salarios y ganancias.

Sin embargo la ecuación $\mathbf{L}=\boldsymbol{K} \boldsymbol{X}$ no describe exactamente lo que dice Sraffa sino la ecuación (a.4) que traemos a colación:

$$
L_{n x 1}^{T}=\underset{n x 1}{K} \otimes(\underset{n x n}{X} \underset{n x 1}{I})
$$

donde $L^{\top}$ es las transpuesta de $\boldsymbol{L}$. Y (a.4) es una expresión matricial de la expresión algebraica que considera Sraffa tal como:

$$
k_{i}=\frac{l_{i}}{\sum_{j=1}^{n} x_{i j}} \quad \forall i=1 \quad \text { an }
$$

Es decir, que para cada mercancía $\boldsymbol{i}$ existe una relación entre los inputs de trabajo directo $l_{i}$ y la suma de todos los procesos que acaban produciendo la misma mercancía. Queremos ahora comprobar qué efecto tiene (a.4) sobre la que hemos deducido también a partir de los supuestos de Sraffa:

$$
P=\frac{(1+g)\left(1+g_{m}\right) w}{g_{m}-g} \times L Y^{-1}
$$

Hallamos ahora la transpuesta de (a.7):

42 Pero ponderados estos coeficientes $\boldsymbol{a}_{\mathbf{s j}}$ por la relación $\mathbf{k}_{\mathbf{s}}$ que mide el inputs de trabajo $\mathbf{s}$ con la suma de todos los procesos que intervienen en la producción del bien $\mathbf{s}$ de acuerdo con (a.4). 
(a.15)

$$
P^{T}=\frac{(1+g)\left(1+g_{m}\right) w}{g_{m}-g} \times\left(Y^{-1}\right)^{T} L^{T}
$$

Y ahora sustituimos (a.4) en (a.15) y obtenemos:

$$
P_{n \times 1}^{T}=\frac{(1+g)\left(1+g_{m}\right) w}{g_{m}-g} \times\left(Y_{n \times n}^{-1}\right)^{T}[\underset{n \times 1}{K} \underset{n \times 1}{\otimes}(X I)]
$$

Por complicado que parezca la ecuación anterior y dado que $Y$ es una matriz diagonal y la tránspuesta de una matriz diagonal-aunque sea inversa a su vez de otra como es el caso- es la matriz diagonal original, (a.16) queda:

$$
P_{n \times 1}^{T}=\frac{(1+g)\left(1+g_{m}\right) w}{g_{m}-g} \times \underset{n \times n}{\left(Y_{n x 1}^{-1}\right)} \underset{n \times 1}{K \otimes}
$$

Un elemento genérico de (a.17), es decir, un precio i cualquiera vendría dado por:

$$
p_{i}=\frac{(1+g)\left(1+g_{m}\right) w}{g_{m}-g} \times \frac{k_{i} \sum_{j=1}^{n} x_{i j}}{y_{i i}} \quad \forall i=1 \quad a \quad n
$$

Y el cociente entre un precio $\boldsymbol{p}_{\mathrm{s}}$ y otro $\boldsymbol{p}_{+}$cualesquiera sería:

(a.19)

$$
\frac{p_{s}}{p_{t}}=\frac{\frac{k_{s} \sum_{j=1}^{n} x_{s j}}{y_{s s}}}{\frac{k_{t} \sum_{j=1}^{n} x_{t j}}{y_{t t}}}
$$

Vemos en (a.19) que la relación de dos precios cualesquiera no depende -en este modelo ${ }^{43}$ - de las variables distributivas ${ }^{44}$ de salarios y ganancias, sino de las

43 No depende porque hemos supuesto que los inputs de trabajo $\mathbf{L}$, los productos finales $\mathbf{Y}$ y los medios empleados $\mathbf{X}$ son datos, pero si todos ellos los consideramos variables -como ocurre en 
relaciones capital/producto y de la relación trabajo/capital, que está representada por $\boldsymbol{k}$ de acuerdo con la hipótesis (a.4). Lo anterior lo sería en la producción simple. Para la producción conjunta ya no tendríamos solo un elemento de $\boldsymbol{Y}$ como ocurre en la ecuación anterior, sino que la solución sería:

$$
\frac{p_{s}}{p_{t}}=\frac{\frac{k_{s} \sum_{j=1}^{n} x_{s j}}{\sum_{s j} z_{s j} x_{t j}}}{\frac{k_{j}^{n}}{\sum_{j=1}^{n} z_{t j}}} \quad \forall s, t=1 \quad a \quad n
$$

donde-como en el caso anterior- $\mathbf{z}_{\mathrm{ij}}$ representa la posición del elemento ij en la inversa de $Y$. En realidad los sumatorios recogen lo que señala Sraffa con ahínco, que tan importante son los efectos directos de las relaciones capitatrabajo como las relaciones indirectas. Y en el caso de que todas las relaciones capital-trabajo fueran constantes $\left(\boldsymbol{k}=\boldsymbol{k}_{1}=\boldsymbol{k}_{2}=\ldots=\boldsymbol{k}_{\mathrm{n}}\right)$, que sería el caso de las relaciones neoclásicas (de forma análoga, en las marxistas serían las composiciones orgánicas de capital), (a.20) quedaría:

$$
\frac{p_{s}}{p_{t}}=\frac{\frac{\sum_{j=1}^{n} x_{s j}}{\sum_{j=1}^{n} x_{s j}}}{\sum_{j=1}^{n} z_{t j}} \quad \forall s, t=1 \quad a \quad n
$$

En este caso de producción conjunta podemos considerar que la inversa de los sumatorios de $\mathbf{z}_{\mathrm{ij}}$ son las ponderaciones de los sumatorios de los $\mathbf{x}_{\mathrm{sj}}$ y $\mathbf{X}_{\mathrm{ij}}$. 


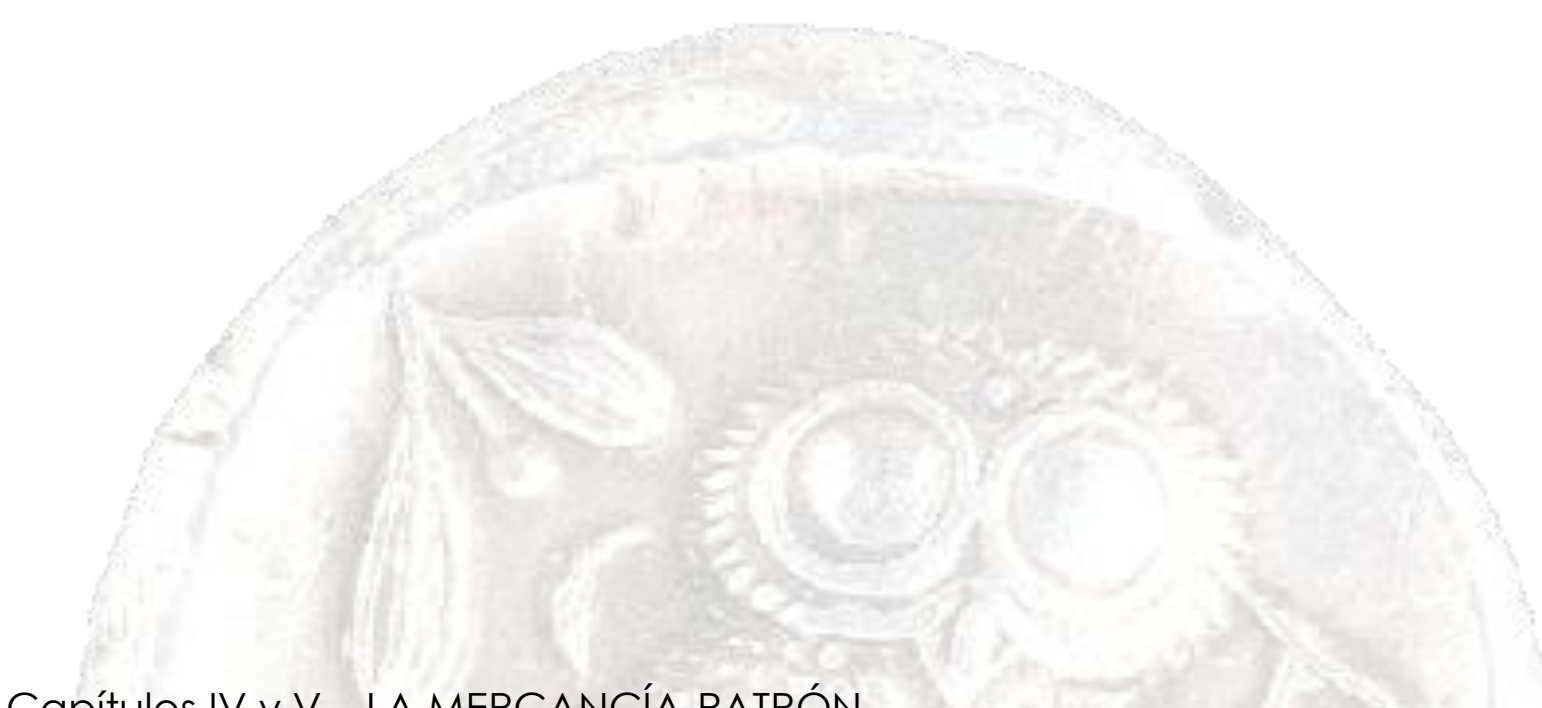

\section{Capítulos IV y V-LA MERCANCÍA-PATRÓN}

I - Nos adentramos en uno de los aspectos más interesantes y originales de su obra y donde más necesaria es meter la cuña de las matemáticas por motivos que se verán. Traemos a colación las palabras directamente de Sraffa que deberían justificar este capítulo: "La necesidad de tener que expresar el precio de una mercancía en términos de otra que es elegida arbitrariamente como patrón complica el estudio de los movimientos de precios que acompañan a una variación en la distribución. Resulta imposible decir, ante cualquier fluctuación particular de precios, si surge como consecuencia de las peculiaridades de la mercancía que está siendo medida, o si surge de las peculiaridades de las mercancía adoptada como patrón de medida45". Decía que deberían justificar este capítulo porque la mercancía-patrón en realidad nos va a servir para medir o evaluar al menos el excedente en términos objetivos, es decir, en términos no monetarios. Añadiré un comentario al respecto porque siempre me ha parecido notable y significativa la capacidad de Sraffa por deslizar problemáticas del análisis económico anterior a él a su pensamiento para, con ello, facilitar-la solución de un problema que no es exactamente el que podríamos llamar histórico. Dicho lo cual no me parece reprochable sino todo lo contrario, demuestra que su nivel intelectual estaba muy por encima del nivel de los economistas de su época salvo excepciones (posibles excepciones serían Joan Robinson y Keynes). El que tenga duda de estos deslizamientos puede leer directamente el primer epígrafe de este capítulo IV que tratamos, porque ahora dice que "si pudiéramos descubrir tal mercancía, nos encontraríamos en posesión de un patrón capaz de aislar los

${ }^{45}$ Epígrafe 23 de PMpM. 
movimientos de precios de cualquier otro producto, de modo que pudieran ser observados como en un vacío" 46 . Esa mercancía a la que se refiere Sraffa sería aquella en la que los medios de producción en relación a los productos finales en términos físicos fuera igual a lo largo de la cadena de medios de producción y productos finales que se han dado en el pasado como efectos indirectos en la producción del presente. Podríamos decir que esa mercancíapatrón va a construir Sraffa estaría formada por un conjunto de bienes en la que los excedentes relativos 47 de las distintas mercancías fueran iguales. Es decir, que, por ejemplo, el excedente relativo total en la producción de carbón fuera el mismo que en la producción total de patatas y este el mismo que en el excedente en la producción de merluza, etc. Excedente relativo sería el cociente entre la diferencia por cada bien entre el producto final y lo empleado del mismo como medio y este último como divisor. Todo ello en términos físicos y para el conjunto de la economía, sin que se trate de una relación de causa y efecto directa en el seno de las empresas. Es decir, debería cumplirse la ecuación para la producción simple:

$$
\frac{y_{i}-\sum_{j=1}^{j=n} x_{i j}}{\sum_{j=1}^{j=n} x_{i j}}=\text { constante } \forall i=1 \quad a n
$$

Esto es equivalente a las relaciones capital/producto de la que hablaban los clásicos puesto que entran los mismos elementos, aunque algebraicamente cambiados. Pero tal mercancía -que Sraffa llama standard commodity- no existe ni puede darse en el mundo real porque se trataría de tal casualidad que la desechamos de entrada. Otra cosa es que podamos construirla de forma virtual. La pregunta sería entonces qué interés podría tener para el análisis económico tal cosa. Existe pero no la adelanto. En todo caso ese interés no coincide enteramente con lo expresado por Sraffa, lo cual hace más interesante todo el tema. Mostraremos que si la economía funcionara con la condición de la ecuación (4.1), ocurriría que, si utilizáramos como numerario tal mercancía, se podría aislar los efectos monetarios de una variación de los precios y salarios de las variables reales como son los inputs de trabajo, los medios de producción y los productos finales. En realidad esto ya lo hemos hecho sin necesidad de la mercancía-patrón con las ecuaciones (3.11) y (3.12), pero nos falta un pequeño detalle que va a aportar este capítulo.

\footnotetext{
46 El deslizamiento se produce ahora porque Sraffa ahora no considera el problema de la distribución y centra la cuestión en encontrar una medida objetiva de las relaciones de intercambio que significan los precios (aunque no solo representen esto sino, también, medida sobre el valor 0 , al algunos casos, costes).

47 Sraffa no emplea esa expresión ni ninguna que pudiera ser traducida como tal, pero creo que recoge fielmente su pensamiento en este punto.
} 
Vista la problemática y las intenciones, vayamos a cómo obtener esa mercancía virtual que tendría la virtud de que los excedentes relativos de todas las mercancías fueran iguales. Para ello partimos -como siempre- de los medios de producción $\boldsymbol{X}$, de los productos finales $\boldsymbol{Y}$ y de los inputs de trabajo $\boldsymbol{L}$-que aparecerán en su momento- jextraídos de la economía real|48! y escribimos por primera vez las ecuaciones matriciales:

$$
\begin{aligned}
& Y Q=(1+R) X Q \\
& L Q=L I
\end{aligned}
$$

donde $\boldsymbol{Q}$ sería un vector vertical de multiplicadores a calcular y $\boldsymbol{R}$ sería la famosa razón-patrón esrafiana, también a calcular. En términos aritméticos (4.2) sería:

$$
\sum_{j=1}^{j=n} y_{i j} q_{j}=(1+R) \sum_{j=1}^{j=n} x_{i j} q_{j} \quad \forall i=1 \text { a } n \quad y \quad \text { con } y_{i j}=0 \text { si } i<>j
$$

Si despejamos $\boldsymbol{R}$ en la anterior se obtiene:

$$
R=\frac{\sum_{j=1}^{j=n} y_{i j} q_{j}-\sum_{j=1}^{j=n} x_{i j} q_{j}}{\sum_{j=1}^{j=n} x_{i j} q_{j}} \quad \text { para todo } i=1 \text { an } \quad \text { y con } y_{i j}=0 \text { si } i<>j
$$

Vemos que (4.5) cumple los mismos criterios que lo que se pedía para una hipotética economía real en (4.1). Visto el contenido, nos ceñimos al aspecto matemático -que es el objeto de este trabajo-porque ahora de lo que se trata es de calcular tanto el vector de multiplicadores $\boldsymbol{Q}$ como la razón-patrón $\boldsymbol{R}$. La primera sorpresa que le deparará al lector del libro de Sraffa es que el economista italiano lo calcula a partir de las ecuaciones (4.2) y (4.3), cuando un cálculo correcto debiera ser de otra forma y, a pesar de ello, los resultados

\footnotetext{
48 Los inputs de trabajo exigirían un esfuerzo de readaptación para hacer equivalente las distintas horas de trabajo según oficios, pero de eso no vamos a hablar. Además este esfuerzo sería el mismo que habría que hacer para la ecuación que intenta modelizar el mundo real que ya hemos visto como el destinado a construir la mercancía-patrón. Por otro lado, dado que Sraffa necesita una ecuación adicional -pero solo una-, no le queda más remedio que mantener el total de inputs de trabajo de la mercancía-patrón $\mathbf{L Q}$ igual al total de inputs del modelo original $\mathbf{L I}$, lo cual es más que arbitrario. Con Perrón-Frobenius, al no necesitar $\mathbf{L}$ para determinar razón y mercancía-patrón, se tiene una absoluta libertad para distribuir los inputs de trabajo entre las diversas actividades, incluso manteniendo el total original. No obstante y abundando en esta libertad que da el teorema, tampoco hay razón para mantener ese total si, por ejemplo, los totales de cada mercancía en la mercancía-patrón han variado respecto al modelo de economía real original.
} 
son los mismos. Veamos. El cálculo correcto sería a partir de la ecuación que surge con apenas manipulación algebraica de (4.2):

$$
\frac{1}{1+R} Q=Y^{-1} X Q
$$

Simplemente hemos traído al primer miembro $\mathbf{1}+\boldsymbol{R}$ y hemos post-multiplicado la ecuación (4.2) por la inversa de $\boldsymbol{Y}$. Cualquier lector versado en las delicias del álgebra matricial reconocerá -viendo (4.6)- que $\boldsymbol{Q}$ y $\boldsymbol{R}$ se obtienen calculando los autovalores y autovectores de la matriz cuadrada y positiva de la matriz resultante $\boldsymbol{Y}^{-1} \boldsymbol{X}$. Ahora bien, de todos esos autovectores $\boldsymbol{Q}$ solo nos interesa aquél que es estrictamente positivo y que se corresponde con el autovalor $(\mathbf{1}+\boldsymbol{R})^{-1}$ más alto en términos absolutos. Para ello vamos a exigir a la matriz resultante $\boldsymbol{Y}^{-1} \boldsymbol{X}$ que cumpla los requisitos del teorema Perron-Frobenius, a saber, que esta matriz sea cuadrada, positiva e irreducible. El primer requisito lo cumple por construcción, el segundo se debe a que estamos en la producción simple y la matriz $Y$ original de productos finales es una matriz diagonal $49, y$ tercero lo cumple por hipótesis, porque vamos a suponer que la matriz $\boldsymbol{Y}^{-1} \mathbf{X}$ es irreducible, es decir, que no se puede triangulizar, es decir, que no se puede, mediante permutaciones de filas o columnas, construir una matriz equivalente donde fueran ceros los elementos por debajo de la diagonal principal o bien por encima ${ }^{50}$. Ya hemos señalado que con el cumplimiento del teorema se resuelven $\boldsymbol{n + 1}$ incógnitas a partir de $\boldsymbol{n}$ ecuaciones. Por ello no hemos necesitado la ecuación ${ }^{51} \mathbf{L Q}=\mathbf{L I}$, que sí necesita Sraffa, la cual es imprescindible en su modelo para igualar incógnitas con ecuaciones, es decir, para resolver el sistema formado por esta última ecuación y la de definición de la mercancíapatrón $Y Q=(1+R) X Q$, lo que le da a Sraffa un conjunto de $\boldsymbol{n}+\mathbf{1}$ ecuaciones con $\mathbf{n + 1}$ incógnitas ${ }^{52}$. Independientemente del método de resolución, la mercancíapatrón estaría formada por el conjunto de vectores resultantes [YQ, XQ, LQ] y la razón-patrón sería $\boldsymbol{R}$, que ya hemos mencionado y que sería aquella que satisfaciera la ecuación matricial $Y \mathbf{Q}=(\mathbf{1}+\mathbf{R}) \mathbf{X Q}$. Por último, el sistema-patrón sería el formado por estos conjuntos de vectores resultantes y la razón-patrón bajo el criterio de que los excedentes relativos sean iguales para todas y cada una de las mercancías.

49 Y por tanto su inversa es positiva porque ésta se forma mediante los inversos de los elementos de la matriz original.

50 Para todo esto recomiendo el excelente apéndice matemático de Lecciones de la Teoría de la Producción, de Luigi Pasinetti.

51 Otra cosa es que se quiera mantener (de forma arbitraria) la igualdad $\mathbf{L Q}=\mathbf{L I}$.

52 Al proceder así se obliga Sraffa a que los inputs de trabajo condicionen las soluciones, cosa que no ocurre aplicando el teorema de Perron-Frobenius. Pero dado que Sraffa no se preocupa ni se ocupa de presentar algo que relacione trabajo y medios de producción, las soluciones que aporta al problema podrían ser entendidas como arbitrarias. Además, con el sistema de solución de Sraffa, nada garantiza que no hubiera soluciones negativas tanto para los precios como para los multiplicadores en el cálculo de la mercancía-patrón. Afortunadamente todo esto puede ser matizado y corregido -como aquí se hace- si es menester y dejar la obra de Sraffa en su grandeza. 
Con el proceder de Sraffa queda determinado cómo se asignan los inputs de trabajo $\mathbf{L Q}$ porque estos se calculan simultáneamente con la ecuación de definición del sistema-patrón, mientras que con Perron-Frobenius la mercancía-patrón se construye jindependientemente de los inputs de trabajo!, como hemos visto. Quizás por este motivo le llevara a Sraffa a considerar que la mercancía-patrón representa una economía en miniatura, lo cual no necesariamente es cierto porque ello va a depender en PerronFrobenius solo exclusivamente de cómo sea la matriz $\boldsymbol{Y}^{-1} \boldsymbol{X}$. También le lleva a la apariencia de que pudiera elegir la reducción de los inputs de trabajo $L$ a esa mercancía en miniatura, lo cual no es cierto porque, como ya hemos dicho, los multiplicadores se calculan simultáneamente con las $\boldsymbol{n}$ ecuaciones de (4.2) y con la ecuación de (4.3). Claro que todo esto se ve facilitado en Sraffa porque no hace ningún súpuesto sobre rendimientos, porque, en ese caso, debería haber añadido un conjunto de ecuaciones ${ }^{53}$ tal como L/XI y que -traducido en mercancía-patrón-sería $\mathbf{L / X Q}$.

II - Llegado a ese punto podría preguntarse el lector que no haya leído la obra de Sraffa para qué sirve todo esto porque, por muchas virtudes que pudiera tener la mercancía-patrón, no nos sirve de nada si se trata de una mera construcción intelectual que cumple un requisito tan peregrino como el de la igualdad de los excedentes relativos para todas las mercancías. Es verdad que el mismo valor teorético tiene la igualdad de las compasiones orgánicas de capital en Marx -es decir, ninguno- o el mismo valor que en los modelos neoclásicos la constancia de la relación capital/trabajo, que es también ninguno, pero parece que con estos últimos se es más indulgente que con los otros. Procedemos a desmentir esta consideración sobre la no utilidad teorética del sistema-patrón y veamos su utilidad principal. Pongamos por un lado la ecuación de definición de una economía real en lo que atañe a la tasa máxima de ganancia $\mathbf{g}_{\mathrm{m}}$, que ya hemos visto:

$$
P Y=\left(1+g_{m}\right) P X
$$

Y comparamos (4.7) con la ecuación de definición de la mercancía-patrón:

$$
Y Q=(1+R) X Q
$$

En principio nada parece tener que ver conceptualmente la tasa máxima de ganancia $\mathbf{g}_{\mathrm{m}}$ con la razón-patrón $\boldsymbol{R}$. Recordamos que la primera es la ganancia que se llevarían los empresarios si los salarios explícitos $\boldsymbol{w}$ fueran cero, todo

\footnotetext{
53 Se escribe así el cociente de dos matrices de forma sintética y ha de entenderse que se quiere expresar así la división, elemento a elemento, de los inputs de trabajo contenidos en cada mercancía entre el total de cada unas de estas mercancías de los medios de producción.
} 
dentro de la economía real; la segunda es el excedente relativo único para todas las mercancías que surge en la construcción de la mercancía-patrón bajo el requisito de que los excedentes relativos de todas las mercancías sean iguales. Conceptos diferentes que corresponden a modelos diferentes: el primero, a pesar de ser un modelo, es un modelo que trata de describir la economía real; el de la mercancía-patrón parece que está buscado a propósito para que no se corresponda con nada de la realidad. Pues bien, vamos ahora a post-multiplicar la ecuación (4.7) por el vector vertical de multiplicadores $\mathbf{Q}$ y la ecuación (4.8) la vamos a pre-multplicar por el vector horizontal de precios $\boldsymbol{P}$. El resultado es:

$$
(P Y) Q=\left(1+g_{m}\right)(P X) Q
$$

$$
P(Y Q)=(1+R) P(X Q)
$$

Y para que se cumplan simultáneamente las dos ecuaciones matriciales anteriores ¡no queda más remedio que $\mathbf{g}_{\mathrm{m}}=\boldsymbol{R}$ ! Y lo que, prima facie, parecía increíble ha sucedido y la tasa máxima de ganancia del modelo real coincide en la producción simple con la razón-patrón de la economía virtual creada por Sraffa. Ahora la ecuación ${ }^{4}$ más famosa de Sraffa, equivalente en su modelo a la ecuación de Einstein de la relación entre masa y energía, es decir, ahora la ecuación del modelo de economía real $\mathbf{g}=(\mathbf{1}-\mathbf{w}) \boldsymbol{g}_{\mathrm{m}}$ que ya hemos visto para la producción simple, podemos sustituirla por $\mathbf{g}=(\mathbf{1}-\mathbf{w}) \boldsymbol{R}$, con lo cual obtenemos una relación entre las variables distributivas que jdepende exclusivamente del excedente! En Sraffa no hay en su modelo determinismo porque $\mathbf{g}$ y $\mathbf{w}$ pueden variar entre sí, dependiente de factores que podemos considerar ajenos al objeto de la economía, pero que no pueden ser arbitrarios porque, fijado una variable, queda fijada la otra.

En lo anterior quizás nos hemos precipitado un tanto porque hemos traído a colación una ecuación como la $\mathbf{g}=(\mathbf{1}-\mathbf{w}) \boldsymbol{g}_{\mathrm{m}}$, cuando en realidad la verdadera ecuación demostrada hasta ahora es $\mathbf{g}=[\mathbf{1}-\mathbf{w} /(\boldsymbol{P}(\boldsymbol{Y}-\boldsymbol{X}) \mathbf{I} / \mathbf{L I})] \mathbf{g}_{\mathrm{m}}$. Sraffa arregló el asunto tomando como numerarios simultáneamente el excedente monetario en términos absolutos $\mathbf{P}(\mathbf{Y}-\mathbf{X}) \mathbf{I}$ y el total de los inputs de trabajo55 $\mathbf{L}$, pero queda en el aire si eso elimina la influencia de los precios en la ecuación trascendental

\footnotetext{
54 Vemos en otra parte que esta ecuación adolece de un error que puede ser subsanado. Lo hacemos en el punto siguiente.

55 El papel que juega LI no es el mismo que el de $\mathbf{P}(\mathbf{Y}-\mathbf{X}) \mathbf{I}$ porque en este último están las variables a determinar que son los precios $\mathbf{P}$ mientras que $\mathbf{L I}$ son datos. Pero Sraffa parte muy hábilmente de que LI vale uno, lo cual es equivalente a haber dividido previamente cada uno de los inputs de trabajo entre el total. Pero ambas consideraciones lleva a que el producto neto y el total del trabajo tengan el mismo valor numérico. La consecuencia de ello es que los precios obtenidos son relativos doblemente, porque lo son sobre el producto neto y sobre los inputs de trabajo, aunque ahora se aporten como un dato y no como variable. En el fondo tomar como numerarios $\mathbf{P}(\mathbf{Y}-\mathbf{X}) \mathbf{I} \mathbf{Y}$, simultáneamente, $\mathbf{L I}$ supone tomar como numerario el cociente entre ambos, lo cual carece de sentido económico. Al menos parece algo estrambótico.
} 
de Sraffa. Tomar como numerario una variable o un dato consiste en dividir jel conjunto de ecuaciones! entre esa variable o dato, lo cual no parece que elimine la influencia, en este caso, de los precios, sino que relativiza esa influencia a los precios en términos de excedente monetario. Pero vamos a solucionar este punto importante en la obra de Sraffa ampliando su modelo con una nueva ecuación, por lo que estamos ya en un territorio de desarrollo conceptual de la obra del economista italiano y no en una mera matematización de su legado o sobre meras especulaciones de lo hecho. Vamos a añadir a las ecuaciones vistas:

$$
P Y=w L+(1+g) P X
$$

$$
P Y=\left(1+g_{m}\right) P X
$$

una tercera que no está en Sraffa pero que está implícita a lo largo de su texto:

$$
P Y=w_{m} L+P X
$$

La anterior nos da, de forma análoga a (4.12) con la tasa máxima de ganancia, la tasa máxima de salarios $\boldsymbol{w}_{\mathrm{m}}$ si se hicieran cero las ganancias en (4.1 1). Pues bien, si eliminamos los precios en el conjunto de las tres ecuaciones matriciales anteriores se obtiene la novedosa pero creo que esrafiana ecuación:

$$
g=\left(1-\frac{w}{w_{m}}\right) g_{m}
$$

¡sin necesidad de tomar ningún numerario que ponga en duda si los precios están incorporados o no al modelo! Puesto de otra manera (4.14) queda:

$$
\frac{g}{g_{m}}+\frac{w}{w_{m}}=1
$$

que es más fácil de recordar. Recordar a su vez ahora que ya hemos demostrado que $\mathbf{g}_{\mathrm{m}}=\boldsymbol{R}$ para la producción simple. 
III - El capítulo $V$ que Sraffa desarrolla resulta un tanto ingenuo a la luz del teorema de Perron-Frobenius, puesto que lo que se intenta es demostrar la unicidad de la razón patrón a partir de intentar demostrar indirectamente que el autovalor más alto dado por $(\mathbf{1}+\boldsymbol{R})^{-1}$ es el único que garantiza que los multiplicadores (autovectores de la matriz $\boldsymbol{Y}^{-1} \boldsymbol{X}$ ) y los precios (los autovectores de la matriz $X Y^{-1}$ ) sean todos positivos. A ambos autovectores les corresponde el mismo autovalor. Además ello da lugar por $(\mathbf{1}+\boldsymbol{R})^{-1}$ a la razón-patrón $\boldsymbol{R}$ más baja. Pero todo esto nos lo da el teorema. Lo que es encomiable, si Sraffa no conocía el teorema, es el esfuerzo por demostrar que tanto multiplicadores y precios han de ser positivos, porque de lo contrario toda la construcción de Sraffa se vendría abajo. Quizás no conocía el teorema pero sí debía tener noticia de algunas de las versiones del teorema del punto fijo porque, por ejemplo, Brouwer ya dio una demostración general en 1912. Y en efecto, contemplando las propias ecuaciones $\mathbf{P Y}=\left(\mathbf{1}+\boldsymbol{g}_{\mathrm{m}}\right) \mathbf{P X}$ y $\mathbf{Y Q}=(\mathbf{1}+\boldsymbol{R}) \boldsymbol{X Q}$ puede verse que es muy posible que el dominio donde se mueven precios $\boldsymbol{P}$ y multiplicadores $\boldsymbol{Q}$ puede representar un espacio convexo y compacto, teniendo en cuenta que nos movemos en el conjunto de los números reales. Además Sraffa siempre parte de que $\boldsymbol{Y}>\boldsymbol{X}$, lo cual facilita enormemente que existan vectores positivos de precios y multiplicadores que cumplan el teorema de Brouwer. En todo caso, de no conocer el teorema de Perron-Frobenius y aún conociendo algunas de las versiones de el del punto fijo, hay que ser un genio y con una seguridad en sí mismo a prueba de bombas como para atreverse a intentar una demostración de lo comentado sin ser un matemático de pura cepa. Recomendaría, no obstante, que en una primera lectura de $\mathbf{P M P M}$ saltarse este capítulo $\vee$ para que no caer en el desánimo.

Terminamos este epígrafe del artículo con un ejemplo numérico del cálculo simultáneo de un vector de precios y otro de multiplicadores para los que se obtiene el mismo autovalor, aunque sus autovectores sean, lógicamente, distintos.

\begin{tabular}{|l|l|l|l|l|}
\hline \multicolumn{5}{|l|}{ Y productos finales } \\
\hline \multirow{2}{*}{ trigo } & proceso 1 & proceso 2 & proceso 3 & sumas \\
\cline { 2 - 5 } hierro & 450 & 0 & 0 & 450 \\
cerdos & 0 & 21 & 0 & 21 \\
\hline
\end{tabular}

\begin{tabular}{|l|l|l|l|l|}
\hline \multicolumn{4}{|l|}{ X= medios } & \\
\hline \multirow{4}{*}{ trigo } & proceso 1 & proceso 2 & proceso 3 & sumas \\
\cline { 2 - 5 } hierro & 186 & 54 & 30 & 270 \\
cerdos & 12 & 6 & 3 & 21 \\
\hline
\end{tabular}




\begin{tabular}{|l|l|l|l|l|}
\cline { 2 - 4 } \multicolumn{4}{c|}{} & \multicolumn{3}{|l|}{ inputs de trabajo } & \multicolumn{1}{l}{} \\
\cline { 2 - 4 } \multicolumn{1}{c|}{} & trigo & hierro & cerdos & total \\
\hline
\end{tabular}

Empleamos de nuevo la programación lineal. La función objetivo es $\mathbf{P Y I}=\mathbf{1}$ y las restricciones exigidas para los precios son que $\mathbf{P Y}=\left(\mathbf{1}+\boldsymbol{g}_{\mathrm{m}}\right) \mathbf{P X}$ y para los multiplicadores $Y \mathbf{Q}=(\mathbf{1}+\boldsymbol{R}) \mathbf{X} \mathbf{Q}$. Por último que $\mathbf{L} \mathbf{Q}=\mathbf{L I}$. Las variables, como es de imaginar, son los $\boldsymbol{n}$ precios $\boldsymbol{P}$, más los $\boldsymbol{n}$ multiplicadores $\boldsymbol{Q}$, más la tasa máxima de ganancia $\boldsymbol{g}_{\mathrm{m}}$ y la razón-patrón $\boldsymbol{R}$. Sraffa peca de optimismo y supone sin demostración alguna que $\boldsymbol{g}_{\mathrm{m}}$ es igual a $\boldsymbol{R}$, pero nosotros nos hemos visto obligados a demostrar que eso es así en párrafos anteriores. El resultado de todo ello es:

\begin{tabular}{|l|l|l|l|l|}
\cline { 2 - 5 } \multicolumn{1}{c|}{} & trigo & hierro & cerdos & PYI \\
\hline $\mathrm{PY}=$ & 0,5996 & 0,2291 & 0,1713 & 1,0000 \\
\hline & & & & \\
\hline
\end{tabular}

\begin{tabular}{|l|l|l|l|}
\hline$Y Q$ & $=$ & $(1+R) X Q$ & $X Q$ \\
\hline 435,6 & & 435,6 & 293,8 \\
35,6 & 35,6 & 24,0 \\
44,5 & & 44,5 & 30,0 \\
\hline
\end{tabular}

Las soluciones son para los precios (tomado como numerario a posteriori el precio del trigo):

\begin{tabular}{|l|l|l|l|}
\hline & trigo & hierro & cerdos \\
\hline precios absolutos $=$ & 0,001332 & 0,010910 & 0,002854 \\
\hline precios relativos $=$ & 1,000 & 8,188 & 2,142 \\
\hline
\end{tabular}

\begin{tabular}{|l|}
\hline$Q$ \\
\hline 0,968 \\
1,695 \\
0,741 \\
\hline
\end{tabular}




\begin{tabular}{|l|l|}
\cline { 2 - 2 } \multicolumn{1}{c|}{} & $\mathrm{Lq}$ \\
\cline { 2 - 2 } & 17,42 \\
& 20,34 \\
$\mathrm{LQ}=$ & 22,24 \\
\hline $\mathrm{LI}=$ & 60,00 \\
\hline
\end{tabular}

Y tanto $\mathbf{g}_{\mathrm{m}}$ como $\boldsymbol{R}$ dan por separado el valor de $\mathbf{4 8 , 2 5 \%}$, y el autovalor correspondiente es de $\mathbf{0 , 6 7 4 5}$.

IV - En todo lo anterior hemos estado trabajando en la producción simple, es decir, bajo un modelo en el que cada mercancía se produce con el mismo método (proceso en Sraffa) y cada proceso produce una sola mercancía. El mundo real parece distinto, prima facie, aunque si meditamos sobre ello tal como hemos hecho y salvo excepciones, el mundo real está mucho más cerca de la producción simple esrafiana de lo que pueda pensarse en un primer momento. No obstante, si partimos de la producción conjunta por mor de un acercamiento al mundo real y por los problemas que nos plantea Sraffa56, deberíamos ser capaces de poder pasar de un modelo de producción conjunta a uno de producción simple equivalente. Ello tendría la ventaja de que, al menos para la construcción de la mercancía-patrón, obtendríamos un vector de multiplicadores siempre positivo y un vector de precios para el modelo de economía real también positivo. Para ello podemos dar al menos dos procedimientos. En el primero podríamos plantear el problema de programación lineal de la manera siguiente: con la función objetivo $\mathbf{L Q}=\mathbf{L I}$, calcular la matriz diagonal de productos finales $\boldsymbol{Y}_{\mathrm{d}}$ de $\boldsymbol{n}$ elementos, los $\boldsymbol{n}$ multiplicadores del vector $\boldsymbol{Q}$ más la razón-patrón $\boldsymbol{R}$, sujeto a las $\boldsymbol{n}$ ecuaciones de $Y \mathbf{Q}=(\mathbf{1}+\boldsymbol{R}) \mathbf{X} \boldsymbol{Q}$ más las $\boldsymbol{n}$ ecuaciones $\mathbf{Y Q}=\boldsymbol{Y}_{d} \boldsymbol{Q}$, donde la matriz $\boldsymbol{Y}_{d}$ es una matriz diagonal. Si recontamos tenemos las mismas ecuaciones que incógnitas (la función objetivo entraña en sí misma una restricción puesto que exigimos un valor concreto y no maximizamos ni minimizamos). Desde luego estos multiplicadores así calculados serán distintos de los calculados directamente con $\mathbf{Y Q}=(\mathbf{1}+\boldsymbol{R}) \mathbf{X} \boldsymbol{Q}$ sin la ecuación $\mathbf{Y Q}=\boldsymbol{Y}_{d} \mathbf{Q}$. La paridad entre ecuaciones e incógnitas se mantiene puesto que también hemos eliminado los $\boldsymbol{n}$ elementos de la matriz $\boldsymbol{Y}_{\mathrm{d}}$.

Otra manera consiste en construir previamente y directamente la matriz diagonal57 $Y_{d}$ a partir de la matriz original $\boldsymbol{Y}$ simplemente colocando en la diagonal principal la suma de los bienes correspondiente a todos los procesos. Es decir, cada elemento de la diagonal principal de $\boldsymbol{Y}_{\mathrm{d}}$ que podríamos llamar

56 Determinación de los costes reales indistinguibles de procesos que producen varias mercancías que son medios de producción y no bienes de consumo finales.

57 Este procedimiento nada tiene que ver con la diagonalización matemática de una matriz a partir de sus autovalores. 


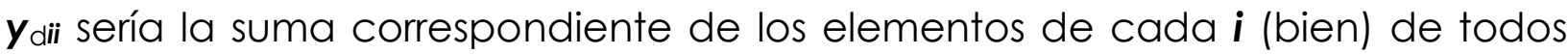
los procesos $\boldsymbol{j}$ de $\boldsymbol{Y}$. Veamos un ejemplo. Supongamos que partimos de una economía definida por el conjunto de matrices de productos finales $Y$, medios $\boldsymbol{X}$ e inputs de trabajo $\boldsymbol{L}$ tal como:

\begin{tabular}{|l|l|l|l|l|}
\cline { 2 - 5 } \multicolumn{1}{c|}{} & \multicolumn{3}{c|}{ Y= productos finales } & \multicolumn{1}{c|}{} \\
\cline { 2 - 5 } \multicolumn{1}{c|}{} & proceso 1 & proceso 2 & proceso 3 & sumas \\
\hline trigo & 55 & 75 & 50 & 180 \\
hierro & 205 & 125 & 115 & 445 \\
cerdos & 165 & 185 & 170 & 520 \\
\hline
\end{tabular}

\begin{tabular}{|l|l|l|l|}
\hline \multicolumn{3}{|l|}{ inputs de trabajo } & \multirow{2}{*}{} \\
\hline trigo & hierro & cerdos & total \\
\hline 187 & 313 & 500 & 1000,0 \\
\hline
\end{tabular}

\begin{tabular}{|c|c|c|c|c|}
\hline & \multicolumn{3}{|c|}{$\mathrm{X}=$ medios } & \\
\hline & proceso 1 & proceso 2 & proceso 3 & sumas \\
\hline trigo & 90 & 50 & 40 & 180 \\
\hline hierro & 120 & 125 & 40 & 285 \\
\hline cerdos & 60 & 150 & 400 & 610 \\
\hline
\end{tabular}

Con lo anterior obtenemos la mercancía-patrón definida por el conjunto de vectores resultantes $Y \mathbf{Q}, \mathbf{X Q}, \mathbf{L}_{\mathbf{Q}} \mathbf{Q}$ tal como:

\begin{tabular}{|c|c|c|c|c|}
\hline & $Y Q$ & $=$ & $(1+R) X Q$ & $\overline{X Q}$ \\
\hline trigo & 229,3 & & 229,3 & 191,4 \\
\hline hierro & 468,4 & 2 & 468,4 & 390,9 \\
\hline cerdos & 597,5 & & 597,5 & 498,7 \\
\hline & & 18 & & \\
\hline & & & & \\
\hline & & & & \\
\hline & & 746,27 & & \\
\hline & & 124,48 & & \\
\hline & & 1000,00 & & \\
\hline
\end{tabular}


Y los multiplicadores $\boldsymbol{Q}$, la razón-patrón $\boldsymbol{R}$ y el autovalor máximo por la derecha correspondientes serían:

\begin{tabular}{|l|l|}
\hline$Q$ & $Q$ \\
\hline & 0,691 \\
2,384 \\
0,249 \\
\hline$R=$ & $19,81 \%$ \\
\hline
\end{tabular}

El autovalor correspondiente calculado a partir de $(\mathbf{1}+\boldsymbol{R})^{-1}$ vale $\mathbf{0 , 8 3 6 5}$. Ahora vamos a calcular multiplicadores, razón-patrón y autovalor a partir de la matriz diagonal de productos finales $\boldsymbol{Y}_{\mathrm{d}}$ tal como:

\begin{tabular}{|l|l|l|l|l|}
\hline \multirow{4}{*}{} & \multicolumn{3}{|l|}{$Y_{d}=$ productos finales } & \multirow{2}{*}{} \\
\cline { 2 - 5 } & proceso 1 & proceso 2 & proceso 3 & sumas \\
\hline trigo & 180 & 0 & 0 & 180 \\
hierro & 0 & 445 & 0 & 445 \\
cerdos & 0 & 0 & 520 & 520 \\
\hline
\end{tabular}

Omitimos dar de nuevo la matriz de medios $\boldsymbol{X}$ y de inputs de trabajo $\boldsymbol{L}$ puesto que son las mismas que las del ejemplo anterior (sólo variamos $Y$ ). Lo que hemos hecho ahora en $\boldsymbol{Y}_{\mathrm{d}}$ es colocar en la diagonal principal la suma por filas (por mercancías) de los elementos de la matriz $Y$. La mercancía así calculada sería:

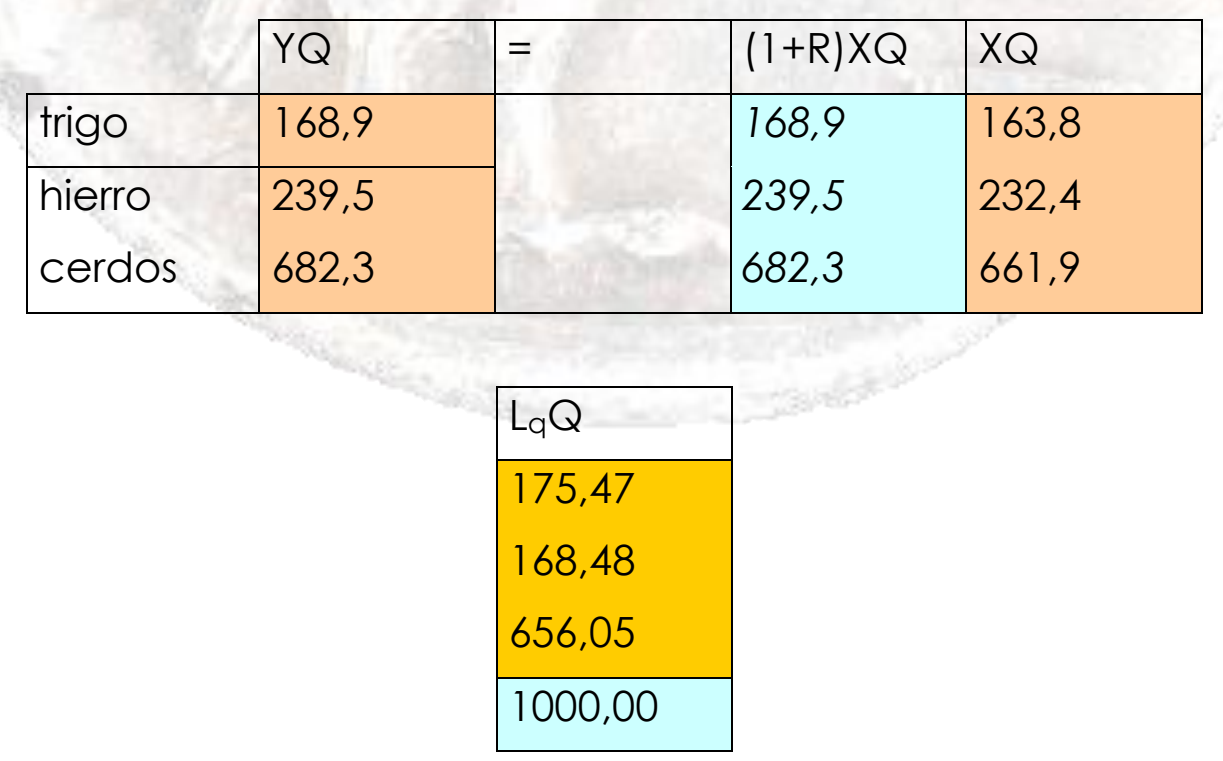

Y los resultados de $\boldsymbol{Q}, \boldsymbol{R}$ y autovalor: 


\begin{tabular}{|l|l|}
\hline \multicolumn{1}{|l|}{} & $Q$ \\
\hline & 0,938 \\
& 0,538 \\
& 1,312 \\
\hline$R=$ & $3,08 \%$ \\
\hline
\end{tabular}

Y el autovalor que le corresponde es 0,9701. Puede observarse que la economía no presenta excedente en todas las mercancías porque el último bien (cerdos) tiene un déficit de $\mathbf{9 0}$ y, a pesar de todo, tanto los multiplicadores como la razón-patrón son positivos ${ }^{58}$.

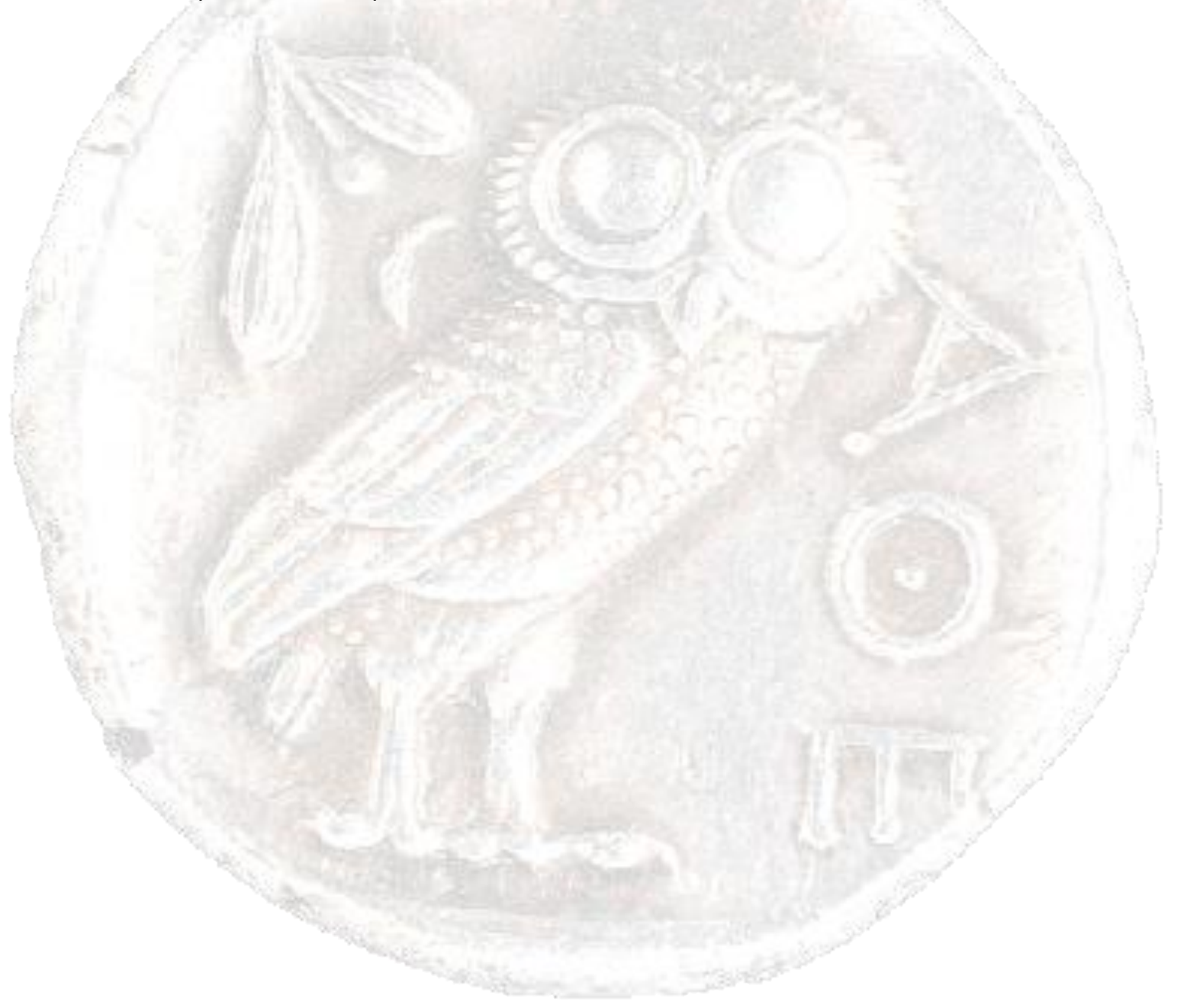

58 Es un error plantear que, para que se de unos multiplicadores positivos y una razón positiva ha de haber excedente para cada uno de los bienes. El teorema de Perron-Frobenius no exige que la matriz $\mathbf{Y}^{-1} \mathbf{X}$ derivada de la ecuación $\mathbf{Y Q}=(\mathbf{1}+\mathbf{R}) \mathbf{X Q}$ sea tal que $\mathbf{Y}>\mathbf{X}$. Otra cosa es que, desde el punto de vista económico, sea conveniente para presentar una economía viable a largo plazo. 


\section{Anexo a al capítulo $V$}

Un conspicuo lector de este capítulo podrá observar que hemos introducido una tercera ecuación a la de definición del sistema y de la tasa máxima de ganancia. Traemos aquí las tres ecuaciones.

$$
P Y=w L+(1+g) P X
$$

$$
P Y=\left(1+g_{m}\right) P X
$$

(a.3)

$$
P Y=w_{m} L+P X
$$

Parecería, según esto, que tenemos más ecuaciones que incógnitas al añadir la $(a, 3)$. Sin embargo no es así porque vamos a demostrar que, bajo ciertas condiciones, podemos hacer que la (a.1) sea una combinación lineal de las otras dos. Demostraremos que el resultado tiene criterio económico y es el mismo que si eliminamos, por ejemplo, los precios entre todas las ecuaciones. Para ese fin vamos a multiplicar (a.2) por un coeficiente a menor que uno pero mayor que cero, y con la (a.3) haremos lo mismo pero con un coeficiente dado por la diferencia 1-a, y, finalmente, sumaremos el resultado de las dos ecuaciones matriciales miembro a miembro. Veamos:

$$
a P Y=a\left(1+g_{m}\right) P X
$$

$$
(1-a) P Y=(1-a) w_{m} L+(1-a) P X
$$

Sumando miembro a miembro queda:

$$
P Y=(1-a) w_{m} L+a\left(1+g_{m}\right) P X+(1-a) P X
$$

Si comparamos (a.6) con (a.2) podemos establecer las siguientes igualdades para que ambas ecuaciones sean iguales:

(a.8) $\quad(1+g)=a\left(1+g_{m}\right)+(1-a)$ 
Ahora despejamos el coeficiente a de ambas y sale:

(a.9)

$$
a=1-\frac{w}{w_{m}}
$$

(a.10)

$$
a=\frac{g}{g_{m}}
$$

Y para que sea lo que queremos, es decir, que (a.1) sea una combinación lineal de (a.2) y de (a.3), las ecuaciones (a.9) y (a.10) están obligadas a ser iguales. El resultado es:

$$
\frac{g}{g_{m}}+\frac{w}{w_{m}}=1
$$

Este es el mismo resultado que si eliminamos, por ejemplo, PYI entre las ecuaciones donde están la tasa máxima de ganancia y la tasa máxima de salarios y sustituimos el resultado en la ecuación de definición del sistema (se nos anula PXI). 
Anexo $b$ al capítulo $V$

I - Cuando estamos en la producción simple ya hemos visto que no tenemos dificultades para construir una mercancía-patrón porque entonces es aplicable el teorema Perrón-Frobenius. Esto permite asegurar un conjunto de multiplicadores estrictamente positivos si se cumplen dos cosas: que la matriz de coeficientes técnicos $\boldsymbol{B}$ que surge de $\boldsymbol{X}=\boldsymbol{Y B}$ en la ecuación $Y \mathbf{Q}=(\mathbf{1}+\boldsymbol{R}) \mathbf{X} \mathbf{Q}$ cumple los requisitos del teorema mencionado; el segundo requisito es que escojamos, de todos los autovalores posibles $(\mathbf{1}+\boldsymbol{R})^{-1}$, aquel cuyo valor es más alto en términos absolutos. La cuestión y el problema surgen si nos vamos de la producción simple y nos adentramos en la problemática producción conjunta. Ahí el teorema vuela y nos quedamos sin asidero formal. Han habido intentos loables y notables de extender la construcción de la mercancía-patrón a la producción conjunta y quizás sea el más notable el de Bertram Schefold en Mr. Sraffa on Joint Production and other essays, 1989. Sin embargo y, en general, adolecen del defecto de anteponer condiciones matemáticas a la economía cuando lo correcto es lo contrario. Y si ello no es posible es mejor seguir otra vía. La dificultad de ello ya la vio el propio Sraffa cuando en el epígrafe 54 del capítulo VIII señala la posibilidad de dos mercancías que puedan ser producidas por dos procesos diferentes y que una de ellas entre en la mercancía-patrón ${ }^{59}$ y la otra no. El problema surge porque en la mercancíapatrón todas las mercancías están representadas en ambos lados de las ecuaciones (todas son básicas), mientras que las no básicas sólo aparecen como productos y no como medios ${ }^{60}$. Vayamos a lo formal. Supongamos que estamos en la producción conjunta y al construir la mercancía-patrón y calcular los multiplicadores bajo la condición de que $Y Q=(1+R) X Q$ ocurra que algunos de los q multiplicadores fueran negativos. Puede ocurrir que la suma de todas las mercancías de los diferentes procesos sea positiva o negativa. Formalmente eso significa que algunos de los elementos de los vectores resultantes $Y \mathbf{Q}, \mathbf{X Q}, \mathbf{L Q}$ puedan ser negativos cuando algún multiplicador $\mathbf{q}$ lo sea también. Pero, dado que ambos vectores resultantes son la suma de todos los $\boldsymbol{y}_{\mathrm{ij}} \boldsymbol{q}_{\mathrm{j}}$ y todos los $\mathbf{x}_{\mathrm{ij}} \boldsymbol{q}_{\mathrm{j}}$ desde $\boldsymbol{j}=\mathbf{1}$ a $\boldsymbol{n}$, puede muy bien ocurrir que los sumandos positivos compensen los negativos y el problema para los productos y medios no exista. Pero, ¿qué pasa con los inputs de trabajo? Estos vienen dados por cada uno de los escalares $\boldsymbol{l}_{\mathbf{j}} \boldsymbol{q}_{\mathbf{j}}$ desde $\mathbf{j}=\mathbf{1}$ a $\boldsymbol{n}$. Aquí no hay suma que valga $y_{\text {, }}$ con tal de que uno de los escalares mencionados valga cero o sea negativo, la construcción de la mercancía-patrón carece de sentido. ¿QQué hacer entonces? Veamos antes de dar la solución un ejemplo de cómo puede ocurrir esto. Sea una economía caracterizada por los productos, medios e inputs de trabajo siguientes:

\begin{tabular}{|c|c|c|c|c|}
\hline & \multicolumn{3}{|c|}{$Y=$ productos finales } & \multirow[b]{2}{*}{ sumas } \\
\hline & proceso 1 & proceso 2 & proceso 3 & \\
\hline bien 1 & 55 & 75 & 50 & 180 \\
\hline
\end{tabular}

59 Ello pasará con seguridad si una de ellas es un producto básico y la otra no.

${ }^{60}$ Con la excepción de las no básicas cuando solo son medios de las propias no básicas. 


\begin{tabular}{|l|l|l|l|l|} 
bien 2 & 210 & 125 & 115 & 450 \\
bien 3 & 165 & 85 & 130 & 380 \\
\hline
\end{tabular}

\begin{tabular}{|l|l|l|l|l|}
\cline { 2 - 5 } \multicolumn{4}{c|}{} & \multicolumn{2}{l|}{ X=medios } & \multicolumn{2}{l|}{} \\
\cline { 2 - 5 } \multicolumn{1}{c|}{} & proceso 1 & proceso 2 & proceso 3 & sumas \\
\hline bien 1 & 90 & 50 & 40 & 180 \\
bien 2 & 120 & 125 & 40 & 285 \\
bien 3 & 60 & 150 & 200 & 410 \\
\hline
\end{tabular}

Pues bien, si construimos la mercancía-patrón a partir de los datos anteriores y con los requisitos consabidos de que $Y Q=(1+R) X Q$ y $L_{s}=L^{6}{ }^{6}$, sale que:

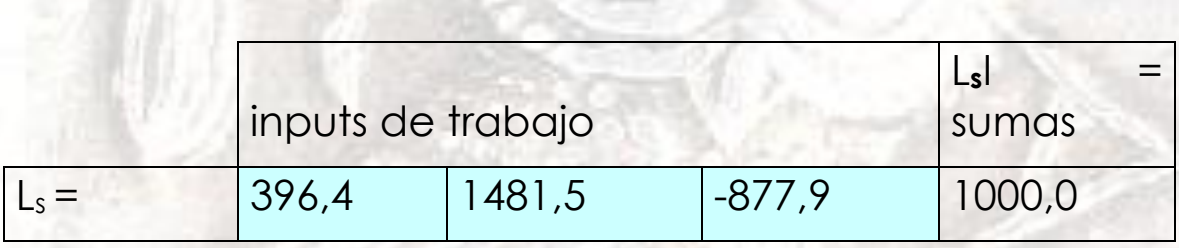

\begin{tabular}{|l|l|l|l|}
\hline $\mathrm{YQ}$ & $=$ & $(1+\mathrm{R}) \mathrm{XQ}$ & $\mathrm{XQ}$ \\
\hline 384,05 & & 384,05 & 357,09 \\
834,67 & & 834,67 & 776,08 \\
523,56 & & 523,56 & 486,81 \\
\hline
\end{tabular}

Y para llegar a lo anterior, los $\boldsymbol{Q}_{\mathrm{s}}$ multiplicadores obtenidos y la razón-patrón correspondiente $\boldsymbol{R}_{\mathrm{S}}$ son:

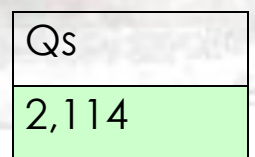

${ }^{61}$ Aceptamos el método de Sraffa de construcción de la mercancía-patrón, lo cual supone que la distribución de los inputs de trabajo es arbitraria puesto que no parte de alguna relación entre trabajo y medios de producción, por ejemplo. Así, si la mercancía-patrón se reduce en términos generales parece lógico que $L_{q}$ se reduzca, pero ¿̇cuánto? Resolveremos esta cuestión más adelante. La única solución lógica -aunque dudosa con criterios económicos- es que se reduzca en función del valor de la razón-patrón $\boldsymbol{R}$, que es lo que se hace cuando se emplea $\mathbf{L} \boldsymbol{Q}$ como componente del trabajo de la mercancía-patrón. Es dudosa porque ello supone asimilar el excedente que representa $\mathbf{R}$ con el tamaño de la mercancía-patrón que representan $\mathbf{Y Q}$ y $\mathbf{X Q}$. 


\begin{tabular}{|l|l|} 
& 4,741 \\
& $-1,756$ \\
\hline$R_{s}=$ & $7,55 \%$ \\
\hline
\end{tabular}

Podemos comprobar que uno de los multiplicadores $(-1,756)$ es negativo. Como se ha visto en el cuadro anterior este multiplicador no es lo suficientemente grande como arruinar los sumatorios de los vectores resultantes $Y Q$ y $\mathbf{X Q}$, pero lo que sí ha arruinado son los inputs de trabajo correspondientes a la tercera mercancía $\left(I_{3} \mathbf{q}_{3}=-877,9\right)$, con to cual el sistema, tal como lo concibe y calcula Sraffa, se viene abajo y no tenemos mercancía-patrón. Volvemos a recordar que, al no aplicar Perrón-Frobenius, Sraffa se ve obligado a utilizar la ecuación matricial $\mathbf{L Q}=\mathbf{L I}$ y de aquí el problema. Nosotros vamos a utilizar para solucionar este problema, no un vector de multiplicadores, sino una matriz de multiplicadores que llamaremos $\boldsymbol{M}$. Para el cálculo de la mercancía-patrón ahora exigiremos que se cumpla que $Y Q=\left(1+R_{g}\right) X Q$ y que $\mathbf{L M I}=\mathbf{L I}$, es decir y para esta última expresión, que la suma de los inputs de trabajo LMI de la mercancía-patrón sea igual a la suma $\mathbf{L I}$ de los inputs originales. Con estas condiciones y a partir de los mismos datos de partida anteriores, sale:

\begin{tabular}{|l|l|l|l|}
\hline YMl & $=$ & $(1+\mathrm{Rg})$ XMI & XMI \\
\hline 384,05 & & 384,05 & 357,09 \\
834,67 & 834,67 & 776,07 \\
523,56 & 523,56 & 486,81 \\
\hline
\end{tabular}

Y ahora-y es la novedad-obtenemos la matriz de multiplicadores $\mathbf{M}$ :

\begin{tabular}{|lll|l|}
\hline \multicolumn{2}{|l|}{ M generalizados } & & MI \\
\hline 2,602 & $-0,326$ & $-0,162$ & 2,114 \\
1,758 & 2,347 & 0,635 & 4,741 \\
$-1,700$ & $-0,720$ & 0,664 & $-1,756$ \\
\hline
\end{tabular}

Puede observarse que los vectores resultantes de ta mercancía-patrón son iguales, es decir, que $\mathbf{Y Q}=\mathbf{Y M I}$ y $\mathbf{X Q}=\mathbf{X M I}$. Sin embargo, lo que ha cambiado es el cálculo de los inputs de trabajo porque, con la nueva matriz de multiplicadores, los inputs de $\mathbf{L M}$ pueden tomar cualquier valor. Por ejemplo, pueden tomar los valores originales haciendo que $\mathbf{L M}=\mathbf{L}$ y sale:

\begin{tabular}{|l|}
\hline$L M$ \\
\hline 187,50 \\
312,50 \\
\hline
\end{tabular}




\begin{tabular}{|l|l|} 
& 500,00 \\
\hline$L M I=$ & 1000,00 \\
\hline
\end{tabular}

Pero estos inputs de trabajo se corresponden con la matriz de multiplicadores $\boldsymbol{M}$ anterior. Ahora supongamos que este vector de inputs de trabajo fuera:

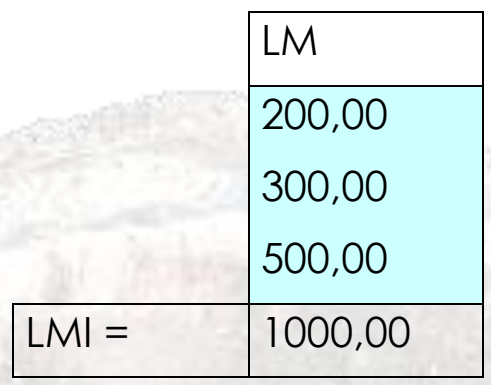

Y, entonces, la matriz de los multiplicadores $\boldsymbol{M}$ sería distinta en sus elementos pero no así en la suma, suma que valdría lo mismo que la anterior y lo mismo que el vector de multiplicadores de la producción simple que ya vimos. Esta nueva matriz valdría:

\begin{tabular}{|l|l|l|l|}
\hline \multicolumn{2}{|l|}{ M generalizados } & MI \\
\hline 2,609 & $-0,332$ & $-0,162$ & 2,114 \\
1,768 & 2,337 & 0,635 & 4,741 \\
$-1,683$ & $-0,736$ & 0,664 & $-1,756$ \\
\hline
\end{tabular}

También son iguales en ambos casos las razones-patrón. Comprobamos que, aunque algunos de los multiplicadores generalizados salgan negativos, no importunan la condición de que la suma de todos los bienes de los diferentes procesos sea positiva en ambos casos, pero lo que cambia sustancialmente son los inputs de trabajo. Con Sraffa no podemos zafarnos de algún inpút negativo $y$, en cambio, con ese método de multiplicadores generalizados funa matriz en lugar de un vector), no sólo tenemos solución positiva para los inputs de trabajo sino que tenemos infinitas soluciones. Es verdad que no podemos construir una mercancía-patrón cuyos inputs de trabajo fueran totalmente peregrinos. Por ejemplo, podemos condicionar la obtención de estos inputs sujeto a que LQ/XQI sean62 iguales o muy parecidos a L/XI. Es verdad que, en este caso, las posibilidades de obtener una mercancía-patrón caracterizada por el conjunto de vectores resultantes $\mathbf{Y M Q}, \mathbf{X M Q}, \mathbf{L Q}$ disminuyen notablemente. Y con esto Y, dado los límites de este trabajo, acabamos esta cuestión.

62 Por supuesto que estos "cocientes" entre vectores resultantes es una manera de indicar que la división lo sería de elemento a elemento que ocupan la misma posición en su vector correspondiente. O dicho de otra manera, sería una división aritmética y no matricial. 
De forma general podemos abordar la mercancía-patrón para la producción conjunta a partir de una ecuación matricial tal como:

$$
Y M I=(1+R) X M I
$$

donde $\boldsymbol{M}$ sería la matriz generalizada de multiplicadores, I un vector vertical de unos, MI el vector vertical de multiplicadores ordinarios (los de Sraffa), el conjunto de vectores dado por $\mathbf{Y M I}, \mathbf{X M I}, \mathbf{L M}$ la mercancía-patrón y $\boldsymbol{R}$ la mercancía-patrón. Si pre-multiplicamos (b.1) por la inversa de $Y$ y, a continuación, pre-multiplicamos también el resultado por el vector horizontal de inputs de trabajo $L$ queda:

$$
L M I=(1+R) L Y^{-1} X M I
$$

Pero ahora imponemos la condición esrafiana de los inputs de trabajo en la mercancía-patrón dada por:

$$
L M I=L I
$$

De entre las ecuaciones matriciales (b.2) y (b.3) sale:

$$
L I=(1+R) L Y^{-1} X M I
$$

Y como quiera siempre es positivo el vector resultante LI (los inputs de trabajo no pueden ser negativos), el resultado en (b.4) es el de que la expresión matemática $\boldsymbol{L Y}^{-1} \mathbf{X} \mathbf{M I}$ ha de ser positiva siempre que se elija $\boldsymbol{M}$ con las condiciones que representan las ecuaciones (b.1) y (b.2), a pesar de que algunos de los multiplicadores generalizados de la matriz $\boldsymbol{M}$ puedan ser negativos (que será lo más probable). Incluso tampoco puede ocurrir que los multiplicadores ordinarios representados por el vector resultante $\mathbf{M I}$ sean negativos. Esto supone un avance respecto a lo planteado por Sraffa porque, si bien no podemos asegurar que -sean cuales sean los datos de productos finales, medios e inputs de trabajo extraídos del mundo real- que se pueda construir la mercancía-patrón para la producción conjunta (en el mundo real hay p. conjunta), sí podemos hacer en la mayoría de los casos en los que, aunque algunos de los multiplicadores ordinarios $\mathbf{M I}$ sean negativos, ocurra que el escalar resultante $\boldsymbol{L Y}^{-1} \boldsymbol{X M I}$ sea positivo. 
Para calcular $\boldsymbol{M}$ lo podemos presentar como un problema de programación lineal donde precisamente la matriz generalizada de multiplicadores $\boldsymbol{M}$ sean con la razón-patrón $\boldsymbol{R}$ las variables a calcular $\left(\boldsymbol{n}^{2+1}\right.$ variables), las restricciones sean que $\mathbf{L M I}=(\mathbf{1}+\boldsymbol{R}) \mathbf{X M I}$, y $\mathbf{L M}=\mathbf{L}$ y la función objetivo que $\mathbf{L M I}=\mathbf{L I}$. En total $\mathbf{2} \boldsymbol{n}+\mathbf{1}$ ecuaciones.

II - En el epígrafe anterior hemos dado un paso hacia delante en la generalización de la mercancía-patrón permitiendo, mediante los multiplicadores generalizados, construir esa mercancía -que también podríamos llamar virtual- a pesar de que algunos de los multiplicadores esrafianos fueran negativos. Lo hecho hasta ahora no se sale, no obstante lo anterior, de la construcción de una mercancía ideal que cumpliera el requisito que todos los excedentes relativos totales de cada mercancía fueran iguales para todas ellas. Es decir, a partir de las ecuaciones $\mathbf{Y M I}=(\mathbf{1}+\boldsymbol{R}) \mathbf{X M I}$ y $\mathbf{L M}=\mathbf{L}$, obtuvimos tres conjuntos de vectores resultantes ${ }^{63}$ [YMI, XMI, LM] y una razón $R$ que definen un sistema-patrón. Sin embargo Sraffa nunca dio el paso de construir una mercancía-patrón para cada uno de las mercancías definidas por $\boldsymbol{Y}, \boldsymbol{X}$ y $\boldsymbol{L}$. Dicho de otra forma, Sraffa construyó la mercancía-patrón para la suma de todas las mercancías del mismo tipo, pero nunca distribuyó esa suma entre cada uno de los elementos definidos por las matrices $\boldsymbol{Y}, \boldsymbol{X}$ y $\boldsymbol{L}$. Y $\boldsymbol{Y}$ eso es lo que vamos hacer en esta parte de este anexo. La forma de hacerlo es sencilla: vamos a repartir proporcionalmente los totales de cada una de las mercancíaspatrón -es decir, repartiremos $\mathbf{Y M I}$ y $\mathbf{X M I}$-entre cada uno de los elementos de $Y, X$ originales de tal manera que, la suma de los nuevos elementos de esta

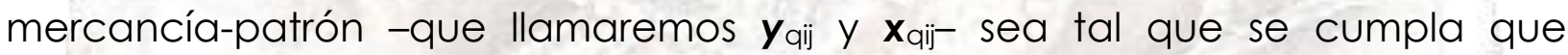

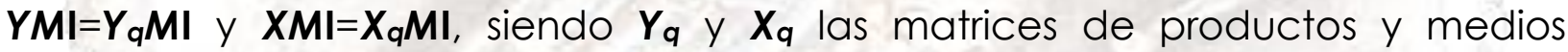
formados por los elementos-patrón (los llamaremos así) anteriores, es decir, por $\mathbf{Y}_{\text {aij }}$ y $\mathbf{x}_{\text {aij. }}$ Estos elementos saldrán con la formulación que sigue:

$$
\begin{aligned}
& y_{q i j}=y_{i j} \times \frac{\sum_{j=1}^{n} \sum_{k=1}^{n} y_{i j} m_{j k}}{\sum_{j=1}^{n} y_{i j}} \quad \forall i=1 \quad a n \\
& x_{q i j}=y_{i j} \times \frac{\sum_{j=1}^{n} \sum_{k=1}^{n} x_{i j} m_{j k}}{\sum_{j=1}^{n} x_{i j}} \quad \forall i=1 \quad a n
\end{aligned}
$$

63 Dado que partimos de la generalización de los multiplicadores, es por lo que usamos el vector resultante $\mathbf{M I}$ post-multiplicando a $\mathbf{Y}$ y a $\mathbf{X}$. Para ir a la mercancía-patrón de Sraffa simplemente lo que hay que hacer es sustituir el producto $\mathbf{M I}$ por el vector de multiplicadores $\mathbf{Q}$, es decir, hacer $\mathbf{M} \mathbf{l}=\mathbf{Q}$, y todo lo que se dice a continuación es válido para el desarrollo del modelo de Sraffa. Pero entonces hay que hacer la salvedad de que solo podemos construir la mercancía-patrón para la producción simple, es decir, solo en el caso de que $\mathbf{Y}$ sea diagonal. En cambio con la matriz $\mathbf{M}$ de multiplicadores generalizados podemos construir esa mercancíapatrón en muchos de los caso de producción conjunta aunque no lo podamos asegurar en todos. 
Como puede comprobarse, si sumamos todos los procesos $\boldsymbol{j}$ de cada mercancía $\boldsymbol{i}$ en ambos lados de la igualdad, queda:

$$
\sum_{j=1}^{n} y_{q i j}=\sum_{j=1}^{n} \sum_{k=1}^{n} y_{i j} m_{j k} \quad \forall i=1 \quad a \quad n
$$

$$
\sum_{j=1}^{n} x_{q i j}=\sum_{j=1}^{n} \sum_{k=1}^{n} x_{i j} m_{j k} \quad \forall i=1 \quad a n
$$

Tanto (b.5) como (b.6) las podemos expresar con matrices mediante:

$$
Y_{q}=Y \otimes[Y M I \div Y I]
$$

(b.10)

$$
X_{q}=X \otimes[X M I \div X I]
$$

Bien entendido que las expresiones indicadas por $\otimes$ y por $\div$ representan productos y divisiones aritméticas y no matriciales, es decir, que multiplican o dividen el elemento ij de la matriz o vector resultante que le precede por el correspondiente ij del vector o matriz que le sucede ${ }^{64}$. Los equivalentes matriciales. Veamos esto con un ejemplo. Partiendo de los datos originales de $Y, X$ y $\boldsymbol{L}$ del epígrafe anterior de este mismo anexo obtuvimos los vectores de la mercancía-patrón $\mathbf{Y M I}$ y $\mathbf{X M I}$ (a falta de los inputs de trabajo que veremos a continuación):

\begin{tabular}{|l|l|l|l|}
\hline YMI & $=$ & $\left(1+R_{\mathbf{g}}\right) \mathrm{XMI}$ & $\mathrm{XMI}$ \\
\hline 384,05 & & 384,05 & 357,09 \\
834,67 & & 834,67 & 776,07 \\
523,56 & & 523,56 & 486,81 \\
\hline
\end{tabular}

Como consecuencia de la definición de mercancía-patrón y para llegar a lo anterior, hemos obtenido el vector de multiplicadores esrafianos $\mathbf{M I}$ y la matriz de multiplicadores generalizados ${ }^{65} \boldsymbol{M}$ tal como:

\footnotetext{
${ }^{64}$ En cambio siguen siendo productos matriciales los vectores resultantes $\mathbf{Y M I}, \mathbf{X M I}, \mathbf{Y I}$ y $\mathbf{X I}$.

${ }^{65}$ Así como la mercancía-patrón dada por YMI y XMI como el vector de multiplicadores $\mathbf{M I}$ son los mismos que los del epígrafe anterior (de donde tomamos los datos), la matriz $\mathbf{M}$ de multiplicadores generalizados no tiene por qué ser la misma. La razón de ello es la de que trabajamos para $\mathbf{M}$ con grados de libertad, cosa que no tenemos para $\mathbf{M l}$. Por ello ocurrirá que, cada vez que calculemos mediante programación lineal la matriz $\mathbf{M}$ sujeta a la restricción de la mercancía-patrón (y cuando la completemos con la restricción de los inputs de trabajo $\mathbf{L M}=\mathbf{L}$ ), variará, pero siempre ocurrirá que la suma por columnas de cada fila de $\mathbf{M}$ (que nos da $\mathbf{M I}$, es decir, el vector esrafiano de multiplicadores) permanecerá constante este vector MI. Si esto no
} 


\begin{tabular}{|lll|l|}
\hline \multicolumn{2}{|l|}{$\begin{array}{l}\text { generalizados } \\
\text { genltiplicadores }\end{array}$} & \multicolumn{1}{l|}{ MI } \\
\hline 0,642 & 0,771 & 0,702 & 2,114 \\
1,475 & 1,691 & 1,575 & 4,741 \\
$-0,753$ & $-0,409$ & $-0,594$ & $-1,756$ \\
\hline
\end{tabular}

Ahora calculamos la re-asignación de los elementos originales $\boldsymbol{y}_{\mathrm{ij}}$ y $\mathbf{x}_{\mathrm{ij}}$ mediante el reparto proporcional de la mercancía-patrón obtenida y sale que:

\begin{tabular}{|lll|l|}
\hline \multicolumn{3}{|l|}{ Yq } & Yql \\
\hline 117,3 & 160,0 & 106,7 & 384,1 \\
389,5 & 231,9 & 213,3 & 834,7 \\
227,3 & 117,1 & 179,1 & 523,6 \\
\hline \multicolumn{3}{|c|}{} \\
\hline 178,5 & 99,2 & 79,4 & 357,1 \\
326,8 & 340,4 & 108,9 & 776,1 \\
71,2 & 178,1 & 237,5 & 486,8 \\
\hline
\end{tabular}

Nos queda ahora la parte de la mercancía-patrón correspondiente a los inputs de trabajo $L$. En la producción simple teníamos el problema de que Sraffa no empleaba Perrón-Frobenius y, por ello, para construir la mercancía-patrón tenía que tirar del total de inputs de trabajo y hacer que $\mathbf{L Q}=\mathbf{L}$, es decir, que, al menos, la suma de los inputs de trabajo de la mercancía-patrón $\mathbf{L Q}$ fuera la misma que la suma original de estos inputs. Ahora, con la producción conjunta, estamos como Sraffa algo desamparados porque ya no podemos emplear el famoso teorema. De alguna manera en la producción conjunta le damos la razón a Sraffa. Pero el problema se agrava si no relacionamos medios con trabajo de alguna manera y la pregunta que surge es: ¿con qué criterio construimos la mercancía-patrón para los inputs de trabajo? Sraffa no tiene criterio puesto que no tiene nada que se le parezca a una relación entre medios y trabajo66. Para solventar el problema de la construcción de la

se entiende no se entenderá la profundidad y el sentido del análisis que hace Sraffa de la mercancía-patrón y la generalización que hemos hecho doblemente: generalización de los multiplicadores y asignación a los elementos de los datos originales de la propia mercancíapatrón.

${ }_{66}$ Sraffa consideraba, creo que con mucha razón, que el capital es solo trabajo fechado. Pero ello le llevó en su modelo a no tener una relación entre los medios que se emplean en la producción y las horas de trabajo empleadas. Y ahora, en la construcción de la mercancíapatrón, se echa de menos. Por ello aquí se va a considerar a que existe esa relación. O de forma equivalente, que a corto plazo aceptamos que la relación entre inputs de trabajo $\mathbf{L}$ y medios empleados en la producción $\mathbf{X}$ es una relación constante (rendimientos constantes de 
mercancía-patrón, su extensión a la producción conjunta y para la reasignación proporcional que se pretende, vamos, al menos, a relacionar y reasignar los inputs de trabajo $\boldsymbol{l}_{i}$ de la matriz original $\boldsymbol{L}$ entre la mercancía-patrón construida con la formulación:

(b.1 1) $l_{q i j}=l_{i j} \times \frac{\sum_{j=1}^{n} \sum_{k=1}^{n} x_{i j} m_{j k}}{\sum_{j=1}^{n} x_{i j}} \quad \forall i=1 \quad a \quad n$

Con los datos originales de este modelo, es decir, con

Bajo estos criterios obtenemos:

\begin{tabular}{|l|l|l|l|l|}
\cline { 2 - 5 } \multicolumn{1}{c|}{} & \multicolumn{3}{|l|}{ Inputs de trabajo } & $\begin{array}{l}\mathrm{Lq}_{\mathbf{q}} \\
\text { sumas }\end{array}$ \\
\hline $\mathrm{Lq}_{\mathbf{q}}=$ & 372,0 & 851,0 & 593,7 & $1.816,6$ \\
\hline
\end{tabular}

Parece lógico que, puesto que la mercancía-patrón resultante emplea más medios de producción que los medios originales (lo mismo para los productos), el total de los inputs de trabajo, independientemente de su distribución entre los diferentes procesos, sea mayor que el total original. Ello se concreta en que $L_{q} I>L I$. Puede comprobarse en lo anterior que no hay inputs de trabajo negativos, cosa que ocurría con la construcción de la mercancía-pátrón con multiplicadores esrafianos y que traigo aquí:

\begin{tabular}{|l|l|l|l|l|}
\hline & \multicolumn{3}{|l|}{ inputs de trabajo } & Ls \\
sumas
\end{tabular}

Con la distribución anterior a esta última hemos resuelto todos los problemas que se planteaban en lo que afecta a los inputs de trabajo: hemos eliminado el valor negativo del último inputs de trabajo $(-877,9)$, hemos construido una matriz de multiplicadores generalizados $\boldsymbol{M}$ que ha dependido tanto de los productos $\boldsymbol{Y}$

forma equivalente si aceptamos, a su vez, que a corto plazo la relación entre productos $\mathbf{Y}$ y medios $\mathbf{X}$ es constante. 
y medios originales como de los inputs de trabajo originales $\boldsymbol{L}$, hemos aumentado los inputs de trabajo acorde con el aumento del tamaño de la mercancía-patrón y, por último, hemos reasignado de forma más racional los propios inputs de trabajo en función de los medios empleados en cada proceso $\boldsymbol{j}$.

\section{Anexo $c$ al capítulo $\mathrm{V}$}

Ya hemos visto en el capítulo anterior que la conexión entre la mercancíapatrón y la razón-patrón es la de que esta última y la tasa máxima de ganancia del modelo de Sraffa coinciden numéricamente en la producción simple ${ }^{67}$. Sraffa no dedujo -aunque lo pudo hacer a partir de sus propios supuestos- que se cumplía que:

$$
g_{m}=R=\frac{L X^{-1}(Y-X) I}{L I}
$$

Pero esta ecuación es inquietante porque tenemos una matriz inversa de la matriz de medios de producción -en la cual todos sus elementos son positivos por motivos obvios-, por lo que no asegura que $\boldsymbol{R}$ sea positivo. La pregunta que nos hacemos es si no habría otra medida de $\boldsymbol{R}$ tal que nos asegurara que tanto tasa máxima de ganancia como razón-patrón sean positivos. Veamos cómo ello es posible. Partimos del modelo de economía real definido por la ecuación:

$$
\underset{1 x n}{P} \underset{n x n}{Y}=(1+g)[w \underset{1 x n}{L}+\underset{1 x n n x n}{P}]
$$

Si de aquí despejamos los precios queda:

$$
P=(1+g) w L[Y-(1+g) X]^{-1}
$$

Si ahora establecemos la ecuación matricial $\boldsymbol{X}=\boldsymbol{A} \boldsymbol{Y}$, siendo $\boldsymbol{A}$ la matriz de coeficientes técnicos que ya hemos visto y, además, desarrollamos la matriz inversa que aparece en el segundo término de la ecuación, quedaría:

$$
P=(1+g) w L Y^{-1}\left[I_{d}+(1+g) A+\ldots+(1+g)^{n-1} A^{n-1}\right]
$$

67 La otra virtualidad de la mercancía-patrón es la que nos va a ayudar en el tema de la reducción del capital a trabajo fechado. 
Pero en lugar de utilizar esta ecuación 68 vamos a hacerlo con otra, donde $\boldsymbol{B}$ va a ser la matriz que sale de $\boldsymbol{B}=\boldsymbol{Y}^{-1} \boldsymbol{X}$ (es decir, de $\boldsymbol{X}=\boldsymbol{Y} \boldsymbol{B}$ ). Si con lo anterior hacemos los cambios oportunos en (c.4) sale:

$$
P=(1+g) w L\left[I_{d}+(1+g) B+\ldots+(1+g)^{n-1} B^{n-1}\right] Y^{-1}
$$

Sraffa aportó, además de la definición de su sistema, la ecuación (c.2) aunque con salarios post-factum (es decir $\mathbf{P Y}=\mathbf{w L}+(\mathbf{1}+\boldsymbol{r}) \mathbf{P X}$ ), pero se dejó a las puertas la que se deduce de su obra y que le hubiera abierto a nuevas situaciones que aquí hemos explorado y lo hacemos de nuevo:

$$
\underset{1 x n}{P} \underset{n x n}{Y}=w_{m} \underset{1 x n}{L}+\underset{1 x n \text { nxn }}{P}
$$

donde $\boldsymbol{w}_{\mathrm{m}}$ es la tasa máxima de salarios. Y a (c.6) le añadimos la que sí aportó:

$$
\underset{1 x n \text { nxn }}{Y}=\left(1+g_{m}\right) \underset{1 x n \text { nxn }}{P}
$$

y que en la producción simple equivale a:

$$
\underset{1 x n \text { nxn }}{P}=(1+R) \underset{1 x n \text { nxn }}{P} \underset{X}{X}
$$

Ello es debido a lo apuntado, a que en la producción simple $\boldsymbol{R}$ es igual a $\mathbf{g}_{\mathrm{m}}$. Pues bien, con las ecuaciones (c.6) y (c.8) obtenemos que los precios valen:

$$
P=\frac{w_{m}}{R} \times L X^{-1}
$$

Al igualar ahora precios entre (c.5) y (c.9) queda la ecuación:

$$
\frac{w_{m}}{R} \times L X^{-1}=(1+g) w L\left[I_{d}+(1+g) B+\ldots+(1+g)^{n-1} B^{n-1}\right] Y^{-1}
$$

68 Quizás no estaría demás aclarar que la sería la matriz diagonal de unos nxn mientras que I sería el vector de unos $\mathbf{n} \times \mathbf{1}$. 
Teniendo en cuenta $\boldsymbol{B}=\boldsymbol{X}^{-1} \boldsymbol{Y}$ que ya hemos definido, post-multiplicando ambos términos de la ecuación por el vector resultante $\mathbf{Y I}$ y despejando además $\boldsymbol{w} / \boldsymbol{w}_{\mathrm{m}}$ sale:

$$
\frac{w}{w_{m}}=\frac{L B I}{(1+g) R L\left[I_{d}+(1+g) B+\ldots+(1+g)^{n-1} B^{n-1}\right] I}
$$

Ya vimos en capítulos anteriores la relación entre salarios y ganancias viene dada por:

$$
g=\frac{w_{m}-w}{w_{m}+w g_{m}}
$$

De la anterior sabemos que, si los salarios $\boldsymbol{w}$ alcanzan a los salarios máximos $\boldsymbol{w}_{\mathrm{m}}$ (por definición no los puede sobrepasar), la tasa de ganancia se hace cero. Pues bien, con esto en la mano y sabiendo que en la producción simple $\mathbf{g}_{\mathrm{m}}=\boldsymbol{R}$ (c.11) queda:

$$
1=\frac{L B I}{R L\left[I_{d}+B+\ldots+B^{n-1}\right] I}
$$

Y despejando la razón-patrón ahora tenemos:

$$
R=\frac{L B I}{L\left[I_{d}+B+\ldots+B^{n-1}\right] I}
$$

Y ipor fin hemos obtenido una razón-patrón (y una tasa máxima de ganancia para la producción simple) como una medida objetiva! no dependiente de ninguna variable monetaria, puesto $\boldsymbol{R}$ que depende solo de las dotaciones iniciales de productos $\boldsymbol{Y}$, de medios $\boldsymbol{X}$ y de los inputs de trabajo $\boldsymbol{L}$. $Y$, además, aquí no tenemos miedo de que la razón-patrón pueda ser negativa puesto que no tenemos ninguna matriz en la igualdad anterior que lo permita. Una curiosidad que surge de (c.14) se derivaría de la pregunta: ¿qué pasaría si estuviéramos en un modelo sin excedente, es decir, en un modelo donde $\boldsymbol{X}=\boldsymbol{Y}$ ? Entonces B=I y la ecuación (c.14) quedaría: 


$$
R=\frac{L I}{L\left[I+I+\ldots+I^{n-1}\right] I}=\frac{L I}{n L I}=\frac{1}{n}
$$

Parecería una contradicción puesto que, si no hay excedente, $\boldsymbol{R}$ debiera ser cero. No hay que preocuparse porque para que $[\mathbf{I}-\mathbf{B}]^{-1}$ sea igual al sumatorio $\mathbf{I}+\boldsymbol{B}+\boldsymbol{B}^{2}+\ldots+\boldsymbol{B}^{\mathrm{n}-1}$ debería extenderse $\boldsymbol{n}$ al infinito 69 , y entonces y solo entonces $\boldsymbol{R}$ sería a cero. Esta es la diferencia entre las consideraciones matemáticas y económicas, porque para que el sumatorio anterior sea igual a $[\mathbf{I}-\mathbf{B}]^{-1}$ ha de extenderse al infinito, lo cual no es aceptable en economía. Eso significa que en el mismo seno del modelo económico tenemos que aceptar que habrá siempre un resto, a yeces no despreciable. No aceptar estos hechos supondría un defecto del modelo económico y no una virtud.

\section{Capítulo VI-REDUCCIÓN (DEL CAPITAL) A TRABAJO FECHADO}

I. Sraffa titula este capítulo como "reducción a cantidades de trabajo fechado" porque no quería que apareciera en su obra la palabra capital por carecer este término de fundamento conceptual y analítico en su modelo. Y, desde luego y también como una crítica más al modelo neoclásicomarginalista. Y no le faltaban razones como ya las dieron en su día Robinson, Kaldor, Garegnani, Pasinetti, etc. Conceptualmente la idea de Sraffa es revolucionaria porque supone dejar al descubierto las incoherencias del modelo neoclásico, además de su poca relación con el mundo real. Sraffa llama medios de producción a lo que se entiende por capital en términos físicos en la jerga neoclásica dominante. Y como siempre el que mejor explica los conceptos es el propio Sraffa y por ello traemos sus palabras en este punto: "Denominaremos reducción a cantidades de trabajo fechadas a una operación mediante la cual, en la ecuación de una mercancía, los diferentes medios de producción son reemplazados por una serie de cantidades de trabajo cada una de las cuales lleva su fecha adecuada". La idea es tan simple como que cualquier medio de producción ha sido producido con trabajo y algún otro medio que, a su vez este último, ha sido producido por otro medio más trabajo, y así podemos retroceder en el tiempo hasta la aparición de los ancestros genéticos de los humanos que al parecer fueron los póngidos. Nada que objetar a lo razonado por Sraffa e incomprensible que en el modelo neoclásico se siga poniendo en pie de igualdad el capital (o medios de producción) y el trabajo. Bien, sin más especulación intelectual, traemos aquí el modelo de definición de una economía esrafiana con salarios pre-factum (en Sraffa son salarios post-factum, pero eso nada cambia para las conclusiones ambas alternativas ${ }^{70}$ ) y tenemos:

$$
P Y=(1+g)[w L+P X]
$$

69 La otra condición es que la serie sea convergente (para lo cual la condición necesaria pero no suficiente es que $\mathbf{B}^{\text {n-1 }}$ tienda a cero).

70 Podemos pasar de los salarios de Sraffa a los pre-factum dividiendo los primeros entre el factor $(\mathbf{1}+\mathbf{g})$ siempre y en todas las circunstancias. 


$$
P=(1+g) w L Y^{-1}+(1+g) P X Y^{-1}
$$

De (6.1) despejamos los precios $\boldsymbol{P}$ y se procede a la sustitución sucesiva del vector de precios de (6.2) en la propia ecuación (en el segundo sumando de la derecha de la ecuación) y queda:

$$
P=(1+g) w L Y^{-1}\left[I+(1+g) A+(1+g)^{2} A^{2}+\ldots+(1+g)^{n-1} A^{n-1}\right]+(1+g)^{n} P A^{n}
$$

Podemos despejar los precios en (6.3), y sale:

$$
P=(1+g) w L Y^{-1}\left\{\sum_{k=0}^{n-1}(1+g)^{k} A^{k}\right\}\left[I-(1+g)^{n} A^{n}\right]^{-1}
$$

En (6.3) vemos que las sucesivas matrices de coeficientes $\boldsymbol{A}^{\mathrm{j}}$ son los medios de producción empleados en el período $\boldsymbol{j}$-1 para producción en el período $\boldsymbol{j}$. El término $(\mathbf{1}+\mathbf{g})^{\text {nPA }}$ P $^{\text {n }}$ que hay en (6.3) es el residuo del que habla Sraffa cuando dice que "Junto a los términos de trabajo siempre habrá residuo de mercancías compuesta de fracciones pequeñas de cada productos básico"71. Dejando aparte el tema de la basicidad que menciona Sraffa, hay que decir que peca -como es habitual- de un cierto optimismo porque ese residuo puede que no sea despreciable porque, si bien $\boldsymbol{A}^{n}$ puede tender a una matriz nula si estamos en una economía muy productiva (que $\boldsymbol{Y}>\boldsymbol{X}$ con diferencia), este hecho puede ser compensado, al menos parcialmente, por el escalar $(\mathbf{1}+\mathbf{g})^{\text {n }}$ si estas tasas de ganancia $\mathbf{g}$ son muy altas (aunque siempre han de estar por debajo $\boldsymbol{R}$ que marca la tasa máxima de ganancia). Por otro lado, el vector resultante $\mathbf{L Y}^{-1}$ mide la inversa de la productividad del trabajo en el momento actual. La conjunción de $\boldsymbol{A}^{\mathrm{j}}$ y $\mathbf{L Y}^{-1}$ mide la participación del trabajo en los medios de producción en cada etapa de la producción. La suma expresada por (6.4) mide, por tanto, los trabajados incorporados en los medios de producción a lo largo del tiempo, pero ponderados de forma sui géneris por el factor $(\mathbf{1 + g})^{\mathrm{j}}$ según en que momento del tiempo nos trasledamos.

II - Pero no nos quedamos aquí. Vamos a llamar $\mathbf{B}$ a la matriz $\boldsymbol{Y}^{-1} \boldsymbol{X}$ y excedente en términos de trabajo ${ }^{72} \mathbf{E}$ a la matriz tal que:

\footnotetext{
71 Epígrafe 46 de PMpM.

72 Esta definición de excedente no es la que hemos visto en el capítulo II de la producción con excedente. Allí la razón-patrón -que es una medida del excedente-surgía de la resolución del sistema de ecuaciones que daba por resultado (6.3) porque la tasa máxima de ganancia $\mathbf{g}_{\mathrm{m}}$ coincidía numéricamente con la razón-patrón $\mathbf{R}$ en la producción simple. Ambas estaban dadas por la expresión $\mathbf{g}_{\mathrm{m}}=\mathbf{R}=\mathbf{L X} \mathbf{X}^{-1}[\mathbf{Y}-\mathbf{X}] / \mathbf{L}$. Insistimos que $\mathbf{R}$ no es pues una definición $-a$
} 


$$
\underset{n x n}{Y=}(I+\underset{n x n}{E}) \underset{n x n}{X}
$$

$$
B=Y^{-1} X
$$

donde I es un vector $\boldsymbol{n} \times \boldsymbol{n}$ de unos en todos sus elementos. Ahora, si efectuamos los productos de la matriz $\boldsymbol{A}\left(=\mathbf{X} \boldsymbol{Y}^{-1}\right)$ en la ecuación matricial (6.3) y agrupamos términos, se obtiene:

$$
P=(1+g) w L\left[I+(1+g) B+(1+g)^{2} B^{2}+\ldots+(1+g)^{n-1} B^{n-1}\right] Y^{-1}
$$

De (6.5) y (6.6) sale que:

$$
B=Y^{-1} X=X^{-1}(I+E)^{-1} X
$$

Vamos a llamar $\mathbf{Z}$ a la matriz que hace que:

$$
Z=X^{-1}(I+E)^{-1} X-I_{d}
$$

La matriz $\mathbf{I}_{d}$ es una matriz diagonal de unos y $\mathbf{Z}$ está formada por números abstractos (sin dimensión) puesto que figura en su producto la inversa de la matriz de los medios de producción $\boldsymbol{X}$ y la matriz original $\boldsymbol{Y}$. Además, los elementos $\boldsymbol{e}_{i j}$ de la matriz también son números abstractos puesto que proceden de (6.5). Con ello obtenemos la matriz B.

$$
B=X^{-1}(I+E) X=(I+Z)^{-1}
$$

Y sustituyendo B de (6.9) en (6.7) queda:

$$
P=(1+g) w L\left\{1+(1+g)(I+Z)^{-1}+(1+g)^{2}\left[(I+Z)^{-1}\right]^{2}+\ldots+(1+g)^{n-1}\left[(I+Z)^{-1}\right]^{n-1}\right\} Y^{-1}
$$
un modelo. 


$$
P=(1+g) w L\left[\sum_{k=0}^{n-1}(1+g)^{k}\left\{(I+Z)^{-1}\right\}^{k}\right] Y^{-1}
$$

En (6.11) hemos logrado reducir las relaciones de capital dadas por $\boldsymbol{A}\left(=\mathbf{X} \boldsymbol{Y}^{-1}\right)$ a trabajado fechado puesto que $\mathbf{Z}$, como vemos en (6.9), es un número sin dimensión y lo que queda en el lado derecho de la ecuación con dimensión es el producto indirecto de $\boldsymbol{L}$ por $\boldsymbol{Y}^{-1}$, que es una medida de la productividad del trabajo (justamente la inversa). Por ello no es difícil encontrar una matriz diagonal, que llamaríamos $\boldsymbol{H}$, calculada ${ }^{73}$ a partir de (6.11):

$$
\underset{1 x n}{P}=(1+g) w\left\{\underset{1 x n}{I}\left[\underset{n x n}{I}+(1+g) \underset{n x n}{H}+\ldots+(1+g)^{n-1} H^{n-1}\right] \otimes \underset{1 x n}{L} Y_{n x n}^{-1}\right\}
$$

$$
P=(1+g) w\left\{\left[\sum_{k=0}^{n-1}(1+g)^{k} \underset{1 \times n n x n}{\operatorname{I}} \underset{1 \times n}{H}\right] \otimes \underset{1 \times n}{L Y^{-1}}\right\}
$$

De forma que hemos colocado en la derecha del corchete de (6.13) los inputs de trabajo cuando estaban a la izquierda en (6.13) y convertida la inversa de $(\mathbf{I}+\mathbf{Z})$ en $\boldsymbol{H}$. En (6.14), el vector resultante podemos considerarlo como las ponderaciones respectivas del vector también resultante $L Y^{-1}$, que representa la inversa de las productividades del trabajo ${ }^{74}$ de cada bien o servicio. El producto del vector del sumatorio por $L Y^{-1}$ es un producto aritmético y no matricial. En definitiva, hemos convertido (utilizando la programación lineal) el producto matricial que hay (6.7) en un producto aritmético que refleja (6.14). Y el propósito de convertir los medios de producción $\boldsymbol{X}$ en trabajo fechado se ha conseguido, puesto que las ponderaciones señaladas son exponenciales, donde el exponente $\boldsymbol{k}$ se refiere al período de tiempo cuando actúa la ponderación (además de incidir en el resultado final en (6.14)).

III - Aunque Sraffa llegara a una ecuación similar a la (6.3), pero omitiendo el último término porque se supone que él lo supone, a su vez, despreciable si nos remontamos en el tiempo, aquí quizás pecó de optimismo porque el factor de compensación de $\boldsymbol{A}^{\mathrm{n}}$ que es $(\mathbf{1}+\mathbf{g})^{\text {n }}$ puede anular al menos parcialmente el anterior. En todo caso no quiso lidiar Sraffa con una ecuación tan compleja como la (6.3), y lo que hace es extraer el término general $\mathbf{w}(\mathbf{1}+\mathbf{g})^{k}$ y sustituir los salarios por la expresión a la que ya había llegado -y de la que

73 Con programación lineal, se igualaría (6.11) con (6.13), lo que serviría como restricción; las variables serían los $\mathbf{n}$ elementos de $\mathbf{H}$, y la función objetivo podría ser minimizar la desviación típica de $\mathbf{H}$.

${ }^{74}$ Serían las equivalentes a las productividades marginales de los modelos neoclásicos, pero con diferencias abismales. Sólo señalar que en este modelo de origen esrafiano no son marginales sino lineales los precios respecto a los inputs de trabajo, salarios y ganancias, y exponenciales respecto al tiempo, lo cual le dota al modelo de un realismo incomparable (al menos en comparación con el marginalismo). 
hemos visto despejada las ganancias- que está dada por $\mathbf{w}=\mathbf{1}-\mathbf{1} / \mathbf{R}$, siendo $\mathbf{R}$ a la vez la razón-patrón y la tasa máxima de ganancia simultáneamente, como hemos demostrado para la producción simple. Entonces le queda el término general (que llamaremos $\operatorname{tg}_{n}$ ) como:

$$
\operatorname{tg}_{k}=\left(1-\frac{g}{R}\right)(1+g)^{k}
$$

Con esta simple ecuación -que es sólo un término de la ecuación generalhace Sraffa algunas disquisiciones sobre los efectos de la reducción del capital a trabajo fechado (6.15) a medida que se mueve la tasa de ganancia $\mathbf{g}$ y $\mathbf{k}$. Esta expresión matemática tiene un máximo con respecto a $\mathbf{g}$ dado que, a medida que aumenta la tasa de ganancia aumenta $(\mathbf{1}+\mathbf{g})^{k}$, disminuye simultáneamente $\mathbf{1 - g} / \boldsymbol{R}$. Se entiende que nunca $\mathbf{g}$ rebasa a $\boldsymbol{R}$, pero si se acerca mucho, la expresión en (6.15) tiende a cero. Además arranca de $\boldsymbol{L}$, con lo cual el gráfico es el que se recoge sobre estas líneas.

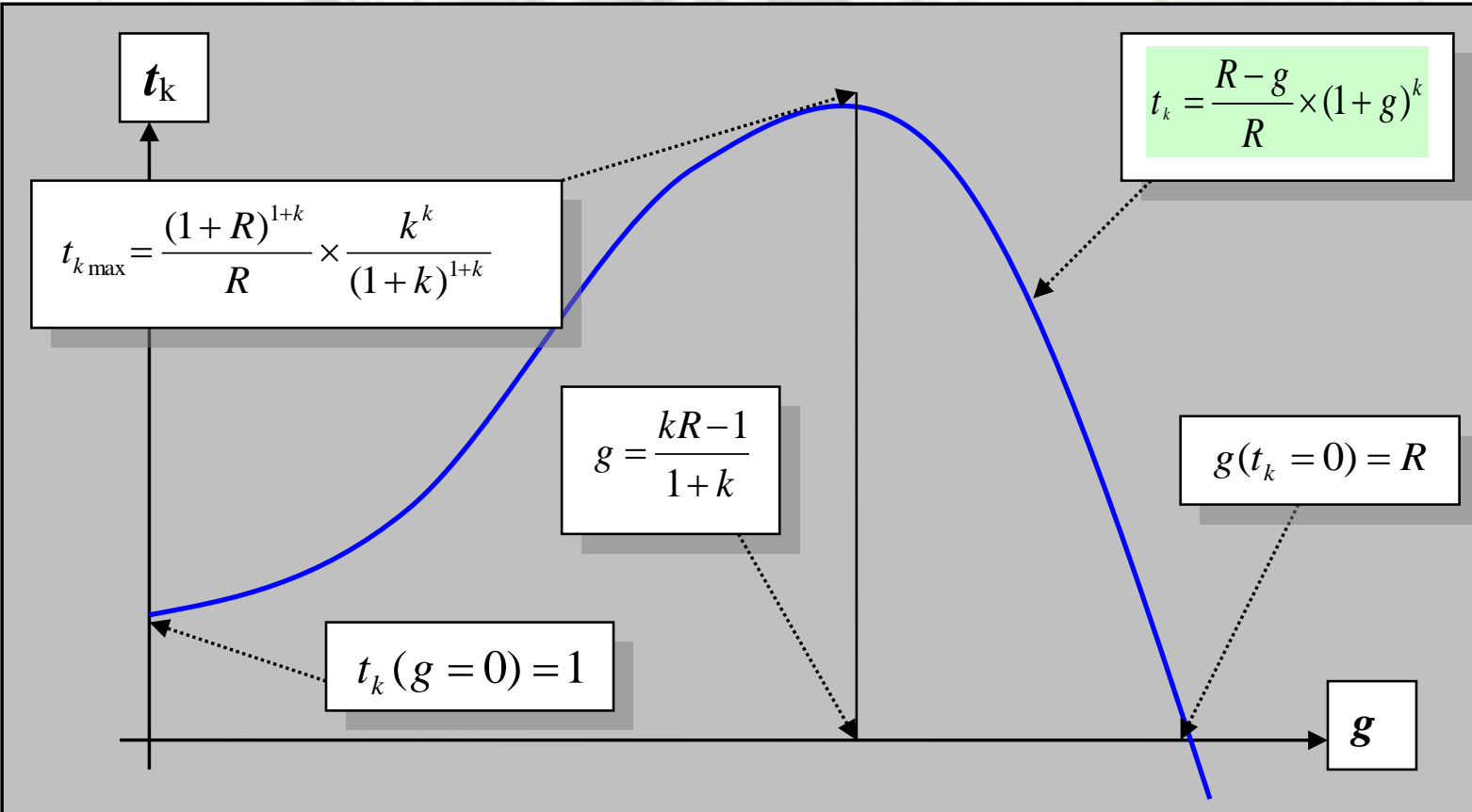

La lectura del capítulo es interesante porque todo lo que escribe Sraffa lo es, pero tiene un interés menor en relación a otros capítulos porque se aparta de posibles reflexiones sobre la reducción del capital a trabajo fechado desde algunas de las posibles ecuaciones generales a las que se puede remitir. Y si Sraffa no lo hace, el autor de este artículo menos, porque solo se trata de ayudar a la lectura de la obra en sus aspectos matemáticos aunque, como ha podido comprobar el lector, también incidimos en aspectos del análisis económico más o menos formalizables. 
IV - Aunque sea una cuestión menor, ayudaremos al lector con esta parte del capítulo de Sraffa. Derivamos la ecuación (53) y la igualamos a cero para llegar al máximo de $\mathbf{t g}_{\mathrm{n}}$, con lo cual obtenemos la tasa de ganancia $\mathbf{g}_{\max }$ que corresponde a ese máximo y el número de períodos $\boldsymbol{k}$ correspondiente:

$$
\begin{gathered}
g_{\text {max }}=\frac{k R-1}{1+k} \\
k=\frac{1+g}{R-g_{\text {max }}}
\end{gathered}
$$

Y lo que parece razonable es que esta tasa de ganancia máxima ${ }^{75}$ sea menor que $\boldsymbol{R}$, que representa todo el excedente y, a la vez, que sea mayor que cero para que el sistema capitalista funcione (sin ganancias y sin expectativas de ganancias el sistema sería difícilmente sostenible). Es decir, que ha de cumplirse que:

$$
0 \leq g_{\max }=\frac{k R-1}{1+k} \leq R
$$

Y para que esta tasa máxima $\mathbf{g}_{\max }$ sea mayor que cero ha de cumplirse que:

$$
1 \leq k R
$$

Lo cual parece bastante razonable. Además, si aumentara indefinidamente el número de períodos $\boldsymbol{k}$, de (6.18) saldría que:

$$
\lim g_{\max }(k \rightarrow \infty) \Rightarrow R
$$

Lo cual es razonable puesto que ninguna medida del excedente puede superar a $\boldsymbol{R}$ porque ello supondría que el modelo describiría una economía inviable.

\footnotetext{
75 Aclarar en todo caso que esta tasa máxima no es la misma que la tasa máxima $\boldsymbol{g}_{\mathrm{m}}$-que venimos utilizando- puesto que la primera está condicionada a la ecuación (6.15) y la habitual surge de hacer cero los salarios en la ecuación de definición del sistema que tantas veces hemos visto.
} 
$\checkmark$ - En el epígrafe II de este capítulo se ha intentado una reducción del capital a trabajo fechado que pueda entenderse un tanto alambicada al recurrir a la programación lineal para encontrar una expresión aritmética que pueda visualizar que esa reducción se lleva a cabo mediante una ponderación. Vamos aquí a dar otra posible versión sin recurrir a la programación lineal. Veamos. Partimos ya directamente de la ecuación:

$$
P=(1+g) w L Y^{-1}\left[I+(1+g) A+(1+g)^{2} A^{2}+\ldots+(1+g)^{n-1} A^{n-1}\right]+(1+g)^{n} P A^{n}
$$

Vamos a considerar que el término $(\mathbf{1}+\mathbf{g})^{n}{ }^{\mathbf{P A}}{ }^{n}$ es despreciable por lo que lo eliminamos de la ecuación y queda:

$$
P=(1+g) w L Y^{-1}\left[I+(1+g) A+(1+g)^{2} A^{2}+\ldots+(1+g)^{n-1} A^{n-1}\right]
$$

$$
P=(1+g) w\left[L Y^{-1}+(1+g) L Y^{-1} A+(1+g)^{2} L Y^{-1} A^{2}+\ldots+(1+g)^{n-1} L Y^{-1} A^{n-1}\right]
$$

Sraffa, en el epígrafe 46 de $\mathbf{P M P M}$ nos da una versión de su reducción donde los vectores resultantes de aquí $L \boldsymbol{Y}^{-1} \boldsymbol{A}^{k}$ han sido reducidos a unos inputs de trabajo LAK que llama "cantidades agregadas de trabajo" sin más explicación. Puede ser suficiente si lo entendemos como que $\mathbf{L Y}^{-1} \boldsymbol{A}^{\mathrm{k}}$ es la cantidad de trabajo incorporado al valor del producto (por unidad) en el período $k$. Y, por lo tanto, la expresión (6.23) recogería la suma de las horas de trabajo (o esfuerzo en general) que, a lo largo del tiempo (desde $\mathbf{k}=\mathbf{0}$ a $\mathbf{k}=\boldsymbol{n}$-1) se han incorporado al producto. Las matrices $\boldsymbol{A}^{\mathrm{k}}$ serían las que recogería las incorporaciones de los distintos sectores que influyen en la producción de un bien o servicio en cada instante del tiempo (o al menos en el período de tiempo convencional del circulante). Ahora bien, para llegar a una solución donde se visualizara que hemos llegado a una reducción completa del capital a trabajo fechado deberíamos llegar a la ecuación:

$$
P=(1+g) w\left\{L_{y 0}+\frac{1+g}{1+R} \times L_{y 1}+\left(\frac{1+g}{1+R}\right)^{2} \times L_{y 2}+\ldots+\left(\frac{1+g}{1+R}\right)^{n-1} \times L_{y n-1}\right\}
$$

En la anterior no hay dificultad para interpretar que los diversos inputs de trabajo $L_{y k}$ que aparecen se pueden corresponder, prima facie, a los vectores resultantes $\left[\boldsymbol{Y}^{-1} \boldsymbol{A}^{\mathrm{k}}\right.$ de la (6.23). La dificultad que se hubiera presentado si esta ecuación si Sraffa no hubiera especulado sobre la llamada mercancía-patrón y la razón-patrón $\boldsymbol{R}$ derivada de lo anterior. La razón de ello es que en (6.24) no podríamos justificar el término $(\mathbf{1}+\boldsymbol{R})$ que aparece en el divisor de los sucesivos 
cocientes $[(\mathbf{1}+g) /(\mathbf{1}+\boldsymbol{R})]^{k}$. Tendríamos en (6.23) una expresión del trabajo fechado, pero sin condicionar sus límites y sin poder apostar por una convergencia de la ecuación. Pero antes de seguir vamos a igualar las expresiones (6.23) y (6.24) igualando sus términos generales:

$$
(1+g)^{k} L Y^{-1} A^{k}=\left(\frac{1+g}{1+R}\right)^{k} \times L_{y k}
$$

Reducción términos y pasando el término $(\mathbf{1}+\boldsymbol{R})^{\mathrm{k}}$ al lado izquierdo de la expresión queda:

$$
L Y^{-1} A^{k}=\left(\frac{1}{1+R}\right)^{k} L_{y k}
$$

Y la verdadera dificultad de la expresión anterior es la constancia del término $\boldsymbol{R}$ y su definición. Lo primero se debe a que lo natural sería que este término fuera variando a lo largo del tiempo, es decir, para cada valor de $\boldsymbol{k}$. Entonces la expresión (6.26) se convertiría en una definición tal como:

$$
L Y^{-1} A^{k}=\prod_{k=0}^{k}\left(\frac{1}{1+R_{k}}\right)^{k} L_{y k}
$$

Además seguiríamos sometidos a la arbitrariedad del valor de $\boldsymbol{R}$. Con la mercancía-patrón podemos obtener $\boldsymbol{R}$ a partir de la ecuación de construcción de esa mercancía virtual que se inventó Sraffa mediante la ecuación:

$$
Y Q=(1+R) X Q
$$

aplicando Perrón-Frobenius a la matriz $\boldsymbol{Y}^{-1} \boldsymbol{X}$, y de ahí, tomando el autovalor más alto en términos absolutos dado por la expresión $(\mathbf{1}+\boldsymbol{R})^{-1}$, obtenemos $\boldsymbol{R}$. La matriz $\boldsymbol{Y}^{-1} \boldsymbol{X}$ nos permite aplicar el teorema porque es cuadrada, positiva (por serlo $\boldsymbol{Y}$ dado que estamos en la producción simple) y la suponemos irreducible. Sabemos, además, que en la producción simple $\mathbf{g}_{\mathrm{m}}=\boldsymbol{R}$, es decir, la tasa máxima de ganancia es igual a esta $\boldsymbol{R}$, que es la razón-patrón de esa construcción virtual. Y sabemos sus límites, porque ya hemos visto que la tasa de ganancia $\mathbf{g}$ no puede superar la tasa máxima de ganancia $\mathbf{g}_{\mathrm{m}}$ porque entonces los precios se irían al infinito de acuerdo con la ecuación: 


$$
P=\left(\frac{1+g}{g_{m}-g}\right) \times w L X^{-1}
$$

Todas estas consideraciones permiten justificar la ecuación (6.24) como reducción del capital a trabajo fechado con la condición (de acuerdo con la ecuación (6.29)) de que $\mathbf{g}$ sea menor $\boldsymbol{R}$ porque, de lo contrario, la ecuación no sería convergente (no cumpliría siquiera la condición necesaria).

\section{Capítulos VIII, VIII y IX - PRODUCCIÓN CONJUNTA}

I - Aún cuando podría parecer lo contrario, las matemáticas implicadas en la producción conjunta son más sencillas que las de la producción simple por una cuestión, irónicamente, desgraciada: que nos quedamos sin el teorema de Perron-Frobenius, lo cual supone que nos quedamos sin garantizar una solución positiva para precios y multiplicadores en el modelo de Sraffa. Veamos el porqué. La producción conjunta presenta dos casos opuestos: se da cuando un mismo proceso produce dos o más mercancías distintas y cuando una misma mercancía es producida por dos o más procesos. Sraffa, en lugar de hablar de procesos, habla, en este capítulo, de industrias, pero eso parece más una concesión para hacer comprensible su análisis a los lectores porque en su época estaba asentado -por más increíble que parezca- modelos en los que una empresa producía un solo producto y que no había dos o más empresas que produjeran el mismo producto76. Y eso lo estaba en contra de toda evidencia. Por ejemplo ahora y entonces, el sector energético tiene muchas maneras de producir un $\mathrm{kw} /$ hora como son los métodos basados en el petróleo, en el carbón, en el viento, la luz solar, los residuos biológicos, etc. Y también contemplaban y contemplamos cómo una misma empresa produce múltiples productos. Otra cosa es si somos fieles al propio Sraffa-a pesar de que en el comienzo de este capítulo VIl dedicado a la producción conjunta él mismo no lo hace- y hablamos de procesos. Podemos pensar y nadar sobre la evidencia de que, aun cuando una empresa produzca muchos productos, podríamos separar los distintos procesos implicados en to referente al hecho material de su producción. Otra cosa será los aspecłos administrativos, fiscales, pago de nóminas, cotizaciones, etc. que tenga que abordar y que podemos considerar de "producción conjunta". Pero si la empresa lleva un control de costes adecuado -incluso sin contabilidad de costes- parece razonable que sean distinguibles los distintos procesos que conllevan los distintos productos. La mayor dificultad es la contraria, es decir, la de un mismo producto fabricado

\footnotetext{
76 Eso ocurre, por ejemplo, en el libro de Thomas J. Sargent -premio Nobel de Economía, 2011Macroeconomic Theory, 1979, donde se hace el supuesto de que "La economía consiste de un elevado número de empresas perfectamente competitivas, cada una de las cuales produce el mismo único bien utilizando la misma función de producción". Y luego no levanta semejante restricción en todo el libro.
} 
mediante diferentes procesos 77 como es el caso energético que hemos visto antes a modo de ejemplo. Dicho lo anterior entramos en materia matemática correspondiente y exponemos la ecuación con salarios pre-factum que definiría la producción conjunta:

$$
P Y=(1+g)[w L+P X]
$$

Viendo sólo matricialmente la ecuación podría parecer que nada ha cambiado, pero sí lo ha hecho porque ahora la matriz $Y$ de productos finales no es una matriz diagonal sino una también $\mathbf{n} \times \boldsymbol{n}$ pero de $\boldsymbol{n} \times \boldsymbol{n}$ elementos. Su expresión aritmética sería:

$$
\sum_{i=1}^{i=n} p_{i} y_{i j}=(1+g)\left[w l_{j}+\sum_{i=1}^{i=n} p_{i} x_{i j}\right] \quad \forall j=1 \quad a \quad n
$$

Sin algún lector del texto pudiera pensar -con razón- que utilizar tasas unitarias de salarios y ganancias nada tiene que ver con la realidad, le acoplamos una ecuación generalista, con $\boldsymbol{n} \times \boldsymbol{n}$ tasas de ganancia $\boldsymbol{B}$ y $\boldsymbol{n} \times \boldsymbol{n}$ tasas de salarios $\boldsymbol{W}$ tal como:

$$
\begin{aligned}
& P Y=L[W \otimes(I+B)]+P[X \otimes(I+B)] \\
& \sum_{i=1}^{n} p_{i} y_{i j}=\sum_{i=1}^{n} l_{i}\left[w_{i j}\left(1+b_{i j}\right)\right]+\sum_{i=1}^{n} p_{i}\left[x_{i j}\left(1+b_{i j}\right)\right] \quad \forall j=1 \quad a \quad n
\end{aligned}
$$

donde los productos internos de las matrices $\boldsymbol{W}$ por las matrices $\mathbf{I}+\boldsymbol{B}$ son productos aritméticos, es decir, que el elemento ij de $\boldsymbol{w}$, es decir, $\boldsymbol{w}_{\mathrm{ij}}$, se multiplica solo por la suma resultante $\mathbf{1}+\boldsymbol{b}_{\mathrm{ij}}$; lo mismo se hace con la matriz $\boldsymbol{X}$ de productos finales. El resto de los productos sí son matriciales.

Volviendo a nuestra ecuación con tasas unitarias, la primera dificultad que nos topamos al no poder aplicar el teorema Perron-Frobenius es que ya no tenemos un procedimiento que garantice que haya una solución siempre positiva para los precios del sistema. El propio Sraffa y en el primer epígrafe del capítulo VII lo expone diciendo que "mientras las ecuaciones puedan ser satisfechas por soluciones negativas para las incógnitas, solo son practicables aquellos métodos de producción que, en las condiciones efectivamente

77 Porque, como señala Sraffa, si tenemos un mismo producto producido por métodos diferentes y, por tanto, con costes diferentes: ¿qué precio le corresponde? 
dominantes (es decir, al salario dado o al tipo de ganancia ${ }^{78}$ dado), solo implican precios positivos". Los economistas formados en el equilibrio general marginalista de origen walrasiano o marshalliano será para ellos una aberración quizás la solución que aporta Sraffa porque estaban y están imbuidos de que los modelos económicos que se utilizan en la economía y que forman parte del acerbo del análisis económico son, en su mayoría, deterministas ${ }^{79}$. También conlleva la componente ideológica de que el sistema teorético debe decirnos cuánto debemos cobrar en forma de salarios y no que diga -como en el modelo de Sraffa- que la relación entre salarios y ganancias presenta al menos un grado de libertad. Pero, en fin esta es otra cuestión que nos aleja del tema que ahí nos ocupa. En la producción conjunta se han intentado soluciones para este problema de la posibilidad de precios negativos. El teorema de Perron-Frobenius nos da las condiciones necesarias y suficientes para determinar cuando un sistema lineal de ecuaciones como el expuesto en (7.1) tiene posibles soluciones positivas. Simplemente no hay garantía, de ahí la salida de Sraffa al problema ${ }^{80}$. Otra cosa distinta es que trabajemos con una ecuación como la (7.2), donde los salarios $\boldsymbol{W}$ y las ganancias $\boldsymbol{B}$ aportan $\mathbf{2} \mathbf{n}^{2}$ variables a determinar. Si a eso les sumamos los $\boldsymbol{n}$ precios de $\boldsymbol{P}$ tenemos $\boldsymbol{n}+\mathbf{2} \boldsymbol{n}^{2}$ variables $y$, sin embargo, $\boldsymbol{n}$ ecuaciones ${ }^{8}$. Aún así no siempre el modelo garantizará precios positivos por las acotaciones razonables desde el punto de vista económico para $\boldsymbol{W}$ y $\mathbf{B}$, pero, eso sí, ampliará con mucho las posibles soluciones con precios positivos. $Y$ ello es imprescindible porque en el mundo real no hay precios negativos si queremos que los modelos se acerquen a la realidad.

II - Y así como en lo anterior estuvo acertado Sraffa con las consideraciones sobre precios positivos, creo que no lo estuvo tanto cuando

78 Luis Ángel Rojo Duque, el gran economista español que tradujo en 1966 el libro, habla de beneficios, pero eso, en el contexto de la obra creo que es inadecuado porque el concepto de beneficio va ligado más a la empresa, mientras que el de ganancia podemos asignarlo más a la idea del reparto del excedente, es decir, a la economía global, cosa mucho más acorde con las intenciones de Sraffa y con lo expuesto en el libro suyo. Bastante hizo el profesor Rojo, no obstante, con traducir el libro en un momento en el que el economista italiano era un perfecto desconocido para la mayor pare de los economistas españoles de la época. Vamos, más o menos como hoy mismo. Por otro lado, hoy tampoco traduciríamos standard commodity por mercancía-patrón y sí por mercancía virtual dado el contexto, aunque esto reconozco que es más discutible. En el caso de los "beneficios" creo que admite poca discusión.

79 Entonces no se había desarrollado la teoría de los juegos (estaba al caer). En cambio en la macro -que desarrollarían el mismo Keynes y Kalecki- el primero ya había introducido la incertidumbre y los animal spirits (en la Teoría General .... 1936).

80 Lo cual demuestra que Sraffa, hasta donde llegó en el conocimiento matemático, entendía las matemáticas a carta cabal.

${ }^{81}$ Estrictamente hablando, al no estar sujeto el modelo a una tasa unitaria de salarios máximos (en general, a tasas unitarias), el teorema de Perron-Frobenius no se puede aplicar tanto para bien como para mal. Ya no es exigible el teorema, pero tampoco nos garantiza su no aplicación precios positivos. Las acotaciones mencionadas son que la matriz de salario tiene que ser menor o igual que la matriz de salarios máximos y, análogamente, que la matriz de ganancias sea menor que la de ganancias máximas. Y las posibilidades de precios negativos disminuyen aún más si establecemos también como variables los medios de producción y los productos finales con las acotaciones pertinentes (han de establecerse a corto plazo relaciones entre medios y productos cuasi-constantes). 
forzó su modelo a que tuviera tantos procesos como mercancías producidas, compensándose, según expone, los procesos que producen más de un producto con los productos que son producidos por más de un proceso. Semejante casualidad es inaceptable. Sraffa que, como se puede comprobar por su libro y aun cuando no era un matemático de formación, entendía perfectamente los problemas de los sistemas lineales de ecuaciones, no se quiso apartar de sus matrices ¡cuadradas! creyendo -aunque no lo dice nunca- que estas son las únicas invertibles. O quizás sabía de otras posibilidades y no las quiso contemplar. Por ejemplo, podemos abordar el tema de la producción conjunta separando la producción de los bienes de consumo de aquellos que, más tarde, en el siguiente período de producción, van a entrar en el modelo como medios de producción. Una ecuación así, con tasas unitarias, sería tal como:

$$
\underset{1 x m}{P} \underset{m x n}{C}+\underset{1 x n n \times n}{P}=(1+g)[w \underset{1 x n}{L}+\underset{1 x n}{P} \underset{n \times n}{X}]
$$

Añadimos, por tanto, a los precios $\boldsymbol{P}$, inputs de trabajo $\boldsymbol{L}$, productos finales $\boldsymbol{Y}$, medios de producción $\boldsymbol{X}$ y tasas unitarias de salarios $\boldsymbol{w}$ y de ganancias $\mathbf{g}$, una matriz de bienes de consumo $\mathbf{C} \boldsymbol{m} \times \boldsymbol{n}$ y el vector correspondiente de precios $\boldsymbol{P}_{\mathrm{C}}$ $1 \times m$. Con este modelo es posible considerar que determinados bienes de consumo adscritos a $\mathbf{C}$ son producidos conjuntamente por otros que, en este modelo, van a ser medios de producción y que los adscribimos a $Y$. ¿̇Pueden despejarse los precios de los bienes de consumo $C$ en función de los precios de los medios de producción $\boldsymbol{X}$ ? Pues eso depende. Dejamos la ecuación (7.5) más expedita mediante:

$$
P_{c} C=(1+g) w L-P[Y-(1+g) X]
$$

Y ahora vamos a post-multiplicar miembro a miembro primero por la transpuesta de $\mathbf{C}$ y, a continuación, por la matriz resultante $\left(\mathbf{C} C^{\top}\right)^{-1}$ y el resultado es:

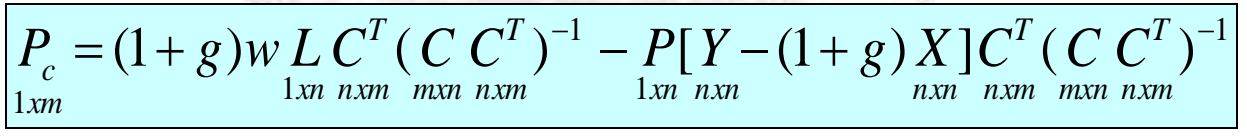

Pues bien, la matriz (CC $\left.\mathbf{C}^{\top}\right)^{-1}$ tendrá inversa si $\boldsymbol{n}$ es mayor que $\boldsymbol{m}$, es decir, si el número de procesos $\boldsymbol{n}$ es mayor o igual que el número de bienes de consumo $\mathbf{m}$ producidos. Y eso significa que en la economía real debiera haber variadas formas de producir un mismo bien, cosa que hemos visto en el ejemplo de la energía. La condición expuesta viene dada por el teorema de RouchéFrobenius y es inapelable. Por otra parte y si se piensa fría y matemáticamente, es razonable porque, de lo contrario y pensando en términos abstractos en la 
teoría de la información, estaríamos exigiendo al sistema más información de que la que partimos. Con este modelo descrito con (7.7) no cabría la posibilidad del caso contrario de producción conjunta, es decir, que hubiera más bienes que procesos utilizados, pero si se piensa con detenimiento eso es más aceptable porque, cualquier producto que se diferencie de otro, por mínimo que sea, siempre exigirá algo distinto que otro por más semejante que sea. Cosa distinta será para la empresa productora la dificultad de separar los costes de cada producto y estos de los costes generales. Sraffa hace interesantes reflexiones sobre estos aspectos que parecían preocuparle sobremanera porque se dio cuenta de que, si no se podía diferenciar los costes por mor de la producción conjunta, las posibilidades de precios negativos serían mayores. El problema para Sraffa es que no empleó ningún instrumental matemático que le ayudara. La realidad es que no lo hay para sus fines y las condiciones de su modelo porque, como queda dicho, sin poder aplicar Perron-Frobenius, nada hay que hacer82. Concretamos este punto. En la producción simple, al despejar los precios en la ecuación de definición del sistema, nos encontramos siempre la matriz resultante $\mathbf{X Y} \boldsymbol{Y}^{-1}$, que, en ese modelo, siempre es positiva porque partíamos de una matriz de medios positiva -lo contrario no tendría sentido económico porque los medios se tienen o no se tienen, no existe medios de producción negativos- y la matriz $Y^{-1}$ era positiva porque la matriz original $Y$ era una matriz diagonal, cosa propia de la producción simple, es decir, la producción que se caracteriza porque cada proceso produce un solo producto y todo producto es producido por un solo proceso. En la producción conjunta eso no es verdad y, ante cualquier caso de producción conjunta de cualquiera de los casos expuestos, la matriz deja de ser diagonal aunque la mayor parte de los elementos que no estén en la diagonal principal sean cero. Por ejemplo, en el caso de un bien producido por dos procesos tendremos colocado en la diagonal principal un coeficiente técnico tal como $\boldsymbol{a}_{\mathrm{ij}}$, pero también habrá en la misma línea horizontal otro coeficiente que tendría un valor distinto de cero y colocado en un espacio de la matriz tal como el (i,j+k), es decir, en la misma fila (mismo bien) pero en distinta columna (distinto proceso). Lo contrario ocurriría si se diera el caso de un mismo proceso (única columna) produciendo varios bienes (varias filas).

82 G. Abraham-Frois y E. Berrebi, en su libro Theory of Value, Prices and Accumulation, 1973, mencionan un artículo de T. Miyao, A generalisation of Sraffa's standard coomodity and its complete characterisation, 1977, se afirma la posibilidad de construir siempre una mercancíapatrón con cantidades positivas. No he podido obtener el artículo, pero, según afirman los autores del libro de referencia, esa posibilidad se debe a que se modifiquen los inputs de trabajo. En efecto, si seguimos a Sraffa y utilizamos las ecuaciones simultaneamente de $\mathbf{Y Q}=(\mathbf{1}+\mathbf{R}) \mathbf{X} \mathbf{Q}$ y $\mathbf{L Q}=\mathbf{L}$, sí cabe la posibilidad de modificar los inputs de trabajo $\mathbf{L}$ (aunque el total sea el de partida como señala la segunda ecuación) de tal manera que, ante un elemento de la columna dada por los vectores resultantes $\mathbf{Y Q}$ y $\mathbf{X Q}$, se podría repartir los inputs de trabajo entre los diferentes bienes de la mercancía-patrón de tal manera que ningún elemento de los dos vectores anteriores fuera negativo. Pero esto chocaría con el inconveniente de que ese reparto no podría ser arbitrario porque ha de mantenerse unas relaciones trabajo/medios de producción dadas 0 , al menos, no arbitrarias. Y el hecho de que Sraffa no haga supuestos sobre rendimientos no significa que, económicamente, las relaciones anteriores puedan ser arbitrarias. Pero como quiera que no tengo el trabajo a la vista, dejo el tema en este punto pendiente de investigación. En todo caso, si los inputs de trabajo son datos o no se utilizan de alguna manera y sólo utilizamos la ecuación de definición de mercancía-patrón como es $\mathbf{Y Q}=(\mathbf{1}+\mathbf{R}) \mathbf{X} \mathbf{Q}$ no se puede soslayar el teorema de Perron-Frobenius. 
III - Desarrolla Sraffa en el capítulo en el capítulo VIII uno de los conceptos que más esfuerzos hizo el economista italiano para convertir en concepto estrella de su obra la diferenciación entre bienes básicos y no básicos. Los primeros serían los que entran como medios de producción en todos los productos finales, y no básicos serían aquellos que, a lo sumo, entran como medio en ellos mismos. Lo explica -aunque no únicamente- en el epígrafe 58 de $\mathbf{P M p M}$. Pero la diferenciación no es sólo ingenieril sino que, además del aspecto de relación física entre medios y productos, también ha de ocurrir que el precio de los bienes básicos no puede depender de los bienes no básicos. Veamos un modelo:

$$
\left[P_{1}, P_{2}\right]\left[\begin{array}{cc}
Y_{11} & Y_{12} \\
0 & Y_{22}
\end{array}\right]=w\left[L_{1}, L_{2}\right]+(1+r)\left[P_{1}, P_{2}\right]\left[\begin{array}{cc}
X_{11} & X_{12} \\
0 & X_{22}
\end{array}\right]
$$

Una ecuación que expresara esta diferenciación estaría representada por (7.8), donde el vector de precios $\boldsymbol{P}_{1}$ y el de inputs de trabajo $\boldsymbol{L}_{1}$ son de rango $\mathbf{1} \times \mathbf{k}$; los vectores $\boldsymbol{P}_{2}$ y $\boldsymbol{L}_{2}$ lo son $\mathbf{1} \times(\boldsymbol{n}-\mathbf{k})$; la matriz de productos $\boldsymbol{Y}_{11}$ es $\mathbf{k} \times \mathbf{k}$ y la matriz $\boldsymbol{Y}_{22}$ es $(\boldsymbol{n}-\boldsymbol{k}) \times(\boldsymbol{n}-\mathbf{k})$; el de $\boldsymbol{X}_{11}$ es $\boldsymbol{k} \times \boldsymbol{k}$, el de $\boldsymbol{X}_{12}$ es $\boldsymbol{k} \times(\boldsymbol{n}-\boldsymbol{k})$ y el de $\boldsymbol{X}_{22}$ es $(\boldsymbol{n}-\boldsymbol{k}) \times(\boldsymbol{n}-\boldsymbol{k})$. Los bienes básicos estarían representados por las submatrices $\boldsymbol{Y}_{11}$ de productos y la $\boldsymbol{X}_{11}$ de medios, la de los no básicos -que solo interactúan entre ellos- estarían representados por el tándem de las submatrices $\boldsymbol{Y}_{22}$ y $\boldsymbol{X}_{22}$. Las submatrices $\boldsymbol{Y}_{12}$ Y $\boldsymbol{X}_{12}$ representarían, a su vez, lo que Sraffa llama productos interconectados, porque se comportan como no básicos porque no influyen en los básicos pero estos sí influyen en aquellos. Si desarrollamos (7.8) queda:

$$
P_{1} Y_{11}=w L_{1}+(1+g) P_{1} X_{11}
$$

$$
P_{1} Y_{12}+P_{2} Y_{22}=w L_{2}+(1+g)\left[P_{1} X_{12}+P_{2} X_{22}\right]
$$

Y en cuanto a los precios vemos que el vector de precios $\boldsymbol{P}_{1}$ isolo! depende de ellos en (7.9) y, en cambio, en (7.10) comprobamos, sin necesidad de despejarlos, que su vector $\boldsymbol{P}_{2}$ depende de ellos mismos y también $\boldsymbol{P}_{1}$ de (7.9), es decir, de los básicos. Despejados los precios en ambas queda:

$$
P_{1}=w L_{1}\left[Y_{11}-(1+g) X_{11}\right]^{-1}
$$

$$
P_{2}=w L_{2}\left[Y_{22}-(1+g) X_{22}\right]^{-1}-P_{1}\left[Y_{12}-(1+g)\left(X_{12}\right)\right]\left[Y_{22}-(1+g) X_{22}\right]^{-1}
$$


donde comprobamos lo expuesto anteriormente.

IV - Aunque no lo vamos a desarrollar, solo apuntar que, al ver los ceros en las matrices de productos y medios en (7.8), se podría caer en la tentación de considerar que basicidad e irreductibilidad son sinónimos. Conceptualmente son cosas diferentes porque irreductibilidad es una propiedad de las matrices que pueden triangulizarse, es decir, las que permiten colocar ceros debajo o encima de la diagonal principal mediante combinaciones lineales de filas y columnas; basicidad, en cambio, es una idea económica que desarrolla Sraffa y que se refiere a los medios de producción que inciden directa o indirectamente en la producción de otros bienes. Sin embargo y mátemáticamente casi son sinónimos. Y decimos casi porque se puede demostrar que puede darse el caso hipotético de que haya irreductibilidad y no basicidad, a pesar de que no sea fácil. Ocurrirá si algunas de las submatrices de los coeficientes técnicos de los bienes básicos dependieran de la submatriz de bienes no básicos, porque entonces el sistema sería reducible pero no habría basicidad por hipótesis. Supongamos que partimos de una matriz donde todos los bienes son básicos, es decir, donde todos contribuyen, directa o indirectamente, a la producción de todos los bienes. Tendríamos una matriz tal como:

$$
\left[P_{1}, P_{2}\right]\left[\begin{array}{ll}
Y_{11} & Y_{12} \\
Y_{21} & Y_{22}
\end{array}\right]=w\left[L_{1}, L_{2}\right]+(1+r)\left[P_{1}, P_{2}\right]\left[\begin{array}{ll}
X_{11} & X_{12} \\
X_{21} & X_{22}
\end{array}\right]
$$

Solo desarrollaremos la primera ecuación de (7.13). Será:

$$
P_{1} Y_{11}+P_{2} Y_{21}=w L_{1}+(1+g)\left[P_{1} X_{11}+P_{2} X_{21}\right]
$$

Si despejamos los precios $\boldsymbol{P}_{1}$ en función del resto queda:

$$
P_{1}=w L_{1}\left[Y_{11}-(1+g) X_{11}\right]^{-1}-P_{2}\left[Y_{21}-(1+g) X_{21}\right]\left[Y_{11}-(1+g) X_{11}\right]^{-1}
$$

Supongamos ahora que hay una dependencia lineal83 entre $\boldsymbol{Y}_{21}$ e $\boldsymbol{Y}_{11}$ tal como $\boldsymbol{Y}_{21}=a \boldsymbol{Y}_{11}$; $\boldsymbol{y}$ entre $\boldsymbol{X}_{21}$ y $\boldsymbol{X}_{11}$ tal que $\boldsymbol{X}_{21}=\mathbf{b} \boldsymbol{X}_{11}$. La ecuación anterior se transforma en:

83 Suponemos además que todas las submatrices son cuadradas y con el mismo número de elementos. 


$$
P_{1}=w L_{1}\left[Y_{11}-(1+g) X_{11}\right]^{-1}-P_{2}\left[a Y_{11}-(1+g) b X_{11} \llbracket Y_{11}-(1+g) X_{11}\right]^{-1}
$$

$Y$, puesto que tenemos solo datos de la submatriz primera, los precios son los correspondientes al vector primero de $\boldsymbol{P}_{1}$, por lo que $\boldsymbol{P}_{2}$ es el mismo vector que $\boldsymbol{P}_{1}$, es decir, la ecuación (7.16) tendrá solución -como cualquier sistema de ecuaciones- si tiene el mismo número de ecuaciones que de incógnitas, razón por la cual $\boldsymbol{P}_{1}=\boldsymbol{P}_{2}$. Si consideramos entonces que las submatrices $\boldsymbol{Y}_{11}$ y $\boldsymbol{X}_{11}$ son irreducibles, se cumpliría que la matriz original también lo es $y$, sin embargo el sistema es básico en su totalidad puesto que hemos supuesto que todos los bienes entran como medio, directa o indirectamente, en todos los demás. De hecho, si despejamos los precios en (7.16) una vez que hemos hecho que $\boldsymbol{P}_{1}=\boldsymbol{P}_{2}$ y manipulamos algebraicamente, sale que:

$$
P_{1}=w L\left[Y_{11}-(1+g) X_{11}\right]^{-1}\left[I+\left[a Y_{11}-(1+g) b X_{11}\right]\left[Y_{11}-(1+g) X_{11}\right]^{-1}\right]^{-1}
$$

Es decir, hemos partido de que estábamos en un modelo donde todos los bienes eran básicos, con lo cual los precios deben depender de todos los $\boldsymbol{n}$ bienes (productos y medios) y (7.18) nos dice que $\boldsymbol{k}$ precios dependen solo de un subconjunto (submatrices $\boldsymbol{Y}_{11}$ y $\boldsymbol{X}_{11}$ ) de $\boldsymbol{k}^{2}$ bienes, mientras que no dependen de los restantes $\mathbf{2} \mathbf{k}(\mathbf{n}-\mathbf{k})+(\mathbf{n}-\mathbf{k})^{2}$ bienes. No obstante, la casualidad en el mundo real de que se dé $\boldsymbol{Y}_{21}=a \boldsymbol{Y}_{11}$ y $\boldsymbol{X}_{21}=\mathbf{b} \boldsymbol{X}_{11}$ es tal que lo podemos considerar un hecho imposible.

\section{Anexo a a la Producción conjunta:}

Lo que viene a continuación no está en Sraffa pero ayudará a entender la diferencia entre basicidad y irreductibilidad una vez más. En 1968 escribió un artículo 84 el economista italiano Carlo Felice Manara que pretendía -entre otros aspectos del artículo- construir una matriz resultante como $\mathbf{Z}$ tal que, a partir de una matriz $\boldsymbol{Y}$ cualquiera de productos finales, $\mathbf{Z}$ tuviera una submatriz de ceros por debajo de la diagonal principal (podría ser por encima). Es decir, dada la matriz $\boldsymbol{Y}$ hallar una matriz $\mathbf{M}$ tal que $\mathbf{Y M}=\mathbf{Z}$. La matriz propuesta por F. Manara sería:

84 Sraffa's Model for the Join Production of Coomodities by means Commodities, L'Industria (en italiano lo presentaba bajo otro título) 


$$
M=\left[\begin{array}{cc}
I_{d} & 0 \\
k x k & k(n-k) \\
T & I_{d} \\
(n-k) k & (n-k) x(n-k)
\end{array}\right]
$$

donde debemos calcular85 $\boldsymbol{T}$, y la matriz resultante $\mathbf{Z}$ debería ser tal que:

$$
\left[\begin{array}{ll}
Y_{11} & Y_{12} \\
Y_{21} & Y_{22}
\end{array}\right]\left[\begin{array}{cc}
I_{d} & 0 \\
T & I_{d}
\end{array}\right]=\left[\begin{array}{cc}
Z_{11} & Z_{12} \\
0 & Z_{22}
\end{array}\right]
$$

donde $\mathbf{I}_{d}$ son matrices diagonales de unos de diferente dimensión (se indica en (a.1)). Lo que se pretende no solo es construir una matriz irreductible tal como la $\mathbf{Z}$, sino que, además, la matriz sea básica, es decir, que la submatriz $\mathbf{Z}_{11}$ no dependa ni de la matriz $\mathbf{Z}_{12}$ ni de $\mathbf{Z}_{22}$. La otra condición es la de que los precios de, por ejemplo, $\mathbf{P Z}=\left(\mathbf{1}+\boldsymbol{g}_{\mathrm{m}}\right) \mathbf{P X} \mathbf{M}$, tengan un subvector $\boldsymbol{P}_{1}$ que solo dependa de la submatriz $\mathbf{Z}_{11}$ y no de los precios $\boldsymbol{P}_{2}$ correspondientes a la submatriz $\mathbf{Z}_{22}$. Sin embargo esta condición no la vamos a tratar porque el paso de la irreductibilidad a la basicidad va a ser cortado antes. Si efectuamos el producto (a.2) obtenemos:

$$
\left[\begin{array}{cc}
Z_{11} & Z_{12} \\
0 & Z_{22}
\end{array}\right]=\left[\begin{array}{cc}
Y_{11}-Y_{12} Y_{22}^{-1} Y_{21} & Y_{12} \\
0 & Y_{22}
\end{array}\right]
$$

Es decir, hemos colocado un cero en la submatriz que correspondería a $\mathbf{Z}_{21}$, con lo cual se cumple la condición necesaria de irreductibilidad. Las condiciones suficientes las damos por hecho porque estas son las de que la submatrices $\mathbf{Z}_{11}$ y $\mathbf{Z}_{22}$ sean irreductibles. Ahora bien, si observamos (a.3) vemos que $\mathbf{Z}_{22}$ depende de $\boldsymbol{Y}_{22}$ (de hecho son la misma submatriz, es decir, en (a.3) $\boldsymbol{Z}_{22}=\boldsymbol{Y}_{22}$. El problema es que $\boldsymbol{Z}_{11}=\boldsymbol{Y}_{11}-\boldsymbol{Y}_{12} \boldsymbol{Y}_{22}{ }^{-1} \boldsymbol{Y}_{12}$ depende, como se ve, de $\boldsymbol{Y}_{22}$ (por esto no habría problema puesto que $\boldsymbol{Y}_{22}$ son los datos originales que son precisamente los que queremos cambiar), pero acabamos de ver que $\boldsymbol{Y}_{22}=\mathbf{Z}_{22}$, por lo que la submatriz resultante queda:

$$
Z_{11}=Y_{12} Z_{22}^{-1} Y_{21}
$$

85 Manara presenta la submatriz $\mathbf{T}$ con valor negativo para que luego, tras calcular su valor según (a.2), se tomara los valores positivos de las submatrices de $\mathbf{Y}$ que intervienen. Es

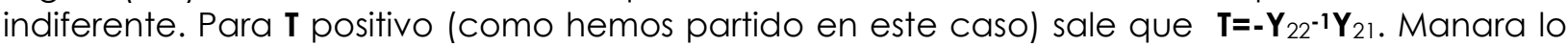

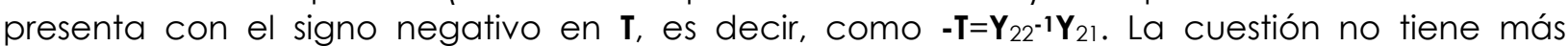
importancia, pero queda la advertencia por si algún estudioso de la obra de Sraffa tuviera acceso al artículo de Manara y se viera sorprendido. 
¡Con lo cual la submatriz $\mathbf{Z}_{11}$, candidata a ser la de los bienes básicos, depende de la submatriz $\mathbf{Z}_{22}$, candidata insoslayable a ser la de los no básicos! La conclusión es la de que, si bien hemos conseguido triangulizar la matriz $Y$ mediante $\mathbf{Z}$, no hemos conseguido hacer independiente los bienes básicos (dados por $\mathbf{Z}_{11}$ ) de los no básicos (dados por $\mathbf{Z}_{22}$ ). No se puede generalizar la matriz auxiliar $\boldsymbol{M}$ porque debemos tener tantas ecuaciones como incógnitas y ello lo hemos conseguido con $\boldsymbol{M}$. En efecto, las submatrices a calcular que son $\mathbf{Z}_{11}, \mathbf{Z}_{12}, \mathbf{Z}_{22}$ y $\boldsymbol{T}_{12}$ tienen $\boldsymbol{n} \times \boldsymbol{n}$ variables, que son las mismas que ecuaciones salen de (c.3). Si a esto le añadimos los precios en la función de precios $\mathbf{P Z}=\left(\mathbf{1}+\mathbf{g}_{\mathrm{m}}\right) \mathbf{P X M}$ ocurre, para más abundancia, que los precios correspondientes a la submatriz $\mathbf{Z}_{11}$ dependen de los precios correspondientes a la submatriz de los bienes no básicos $\mathbf{Z}_{22}$. Lo de F. Manara fue un intento fallido, pero que ilustra (aunque el autor no lo presenta así) la diferencia entre basicidad e irreductibilidad.

\section{Anexo b a la Producción conjunta:}

No se pretende en este trabajo comentar las riquísimas especulaciones que Sraffa lanza sobre su obra pero sí ayudar a su comprensión en los aspectos matemáticos. Este es el caso de este apéndice porque vamos a ayudar a entender al lector el gráfico del epígrafe $\mathbf{7 2}$ del capítulo IX. El dibujo no tiene demasiada dificultad de comprensión pero sí el texto que acompaña al mismo en el eje de ordenadas. En este se coloca al mismo tiempo "el salario" y "el precio patrón", lo cual es imposible puesto que precios y salarios -sea de la mercancía-patrón o no- no vienen medidos en las mismas dimensiones: el salario es un dinero por horas de trabajo y el precio es también un dinero pero por un bien o servicio. Si estuviéramos en Marx y aceptáramos la teoría del valor trabajo, no sólo en su aspecto causal sino también contable, sí podríamos compararlos pero no en Sraffa, donde el propio economista italiano habla de concepción metafísica del valor de Marx. Yendo ya directamente al gráfico, vamos a dar uno equivalente a partir de dos ecuaciones jcomparables!, en las que, en ambas, estarán representadas los salarios en el eje de ordenadas y, al igual que en Sraffa, las ganancias estarían en el eje de abscisas. 
Aunque Sraffa no explicita las ecuaciones, suponemos que una de ellas es la fundamental de distribución con salarios post-factum:

$$
w=1-\frac{g}{R}
$$

Y la otra surge de despejar los salarios en la ecuación fundamental de definición del sistema de Sraffa, también con salarios post-factum:

$$
P Y=w L+(1+g) P X
$$

De la anterior despejamos el vector de precios $\boldsymbol{P}$ y llamados $\boldsymbol{A}$ a la matriz de coeficientes que surge de $\boldsymbol{X}=\mathbf{A} \boldsymbol{Y}$ y tenemos:

$$
P=w L Y^{-1}\left[I+(1+g) A+\ldots+(1+g)^{n-1} A^{n-1}\right]
$$

Y despejando los salarios en (b.3) tras post-multiplicar la ecuación por el vector resultante $Y \mathbf{I}$ queda:

$$
w=\frac{P Y I}{L Y^{-1}\left[I+(1+g) A+\ldots+(1+g)^{n-1} A^{n-1}\right]}
$$

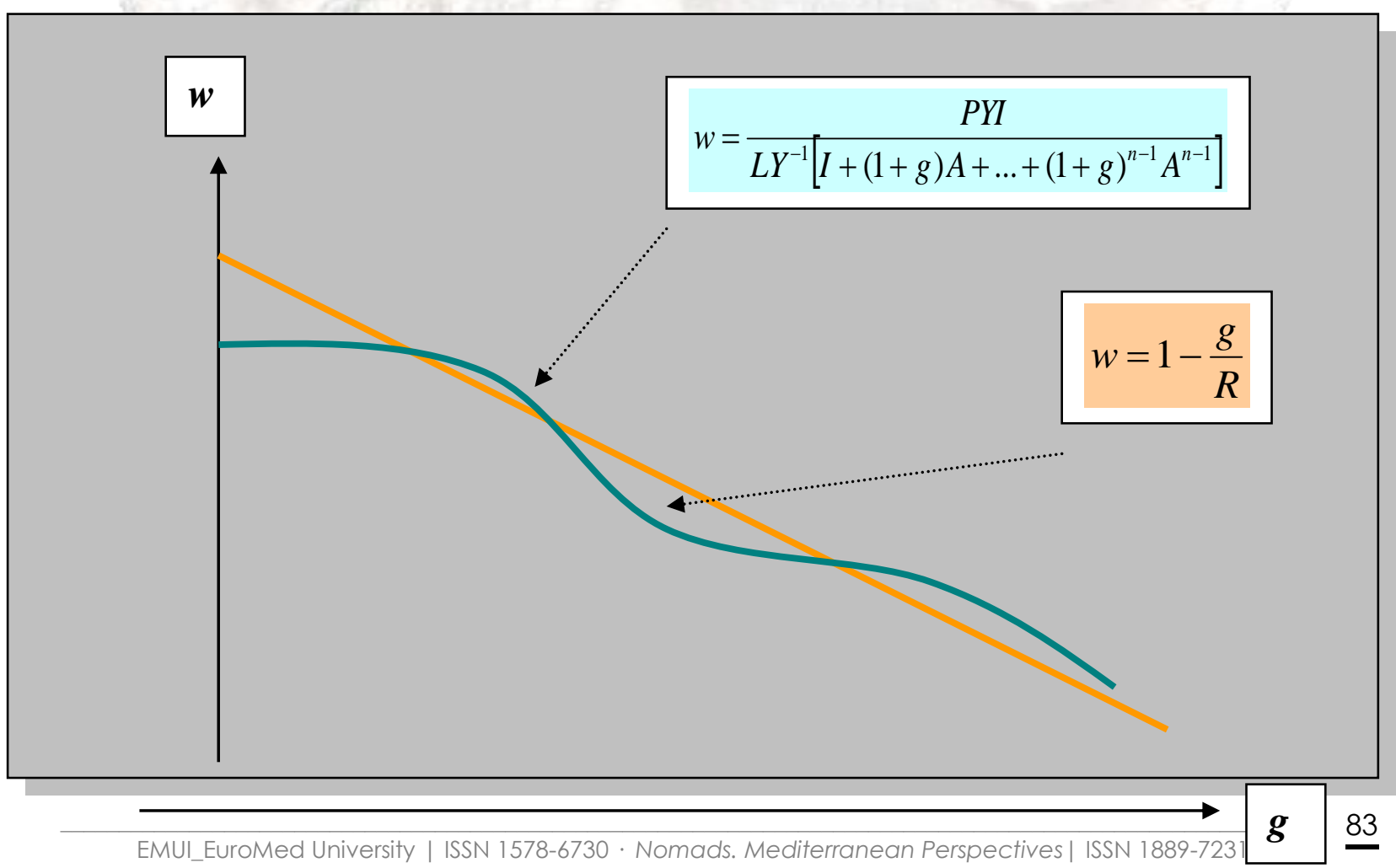




\section{Explicación gráfico del epígrafe 72 de PMpM}

Si igualamos los salarios en las ecuaciones descritas en el gráfico obtendremos los puntos de corte de ambas. Convendría aclarar, en primer lugar, que I es el vector vertical de uno $\mathbf{n \times 1}$ y, además, que hemos empleado la razón patrón $\boldsymbol{R}$ en (b.1) en lugar de la más genérica de tasa máxima de ganancia porque Sraffa nos habla explícitamente de salarios en "términos de la mercancíapatrón"86. Pero hay que señalar que este modelo y la discusión de Sraffa sobre el problema de relacionar salarios y ganancias -es el objeto de discusión de buena parte del capítulo IX-es también válido fuera del sistema-patrón. Y aquí se produce una contradicción porque la ecuación es sólo válida en la producción simple y no en la conjunta. La razón de ello es que hemos tomado -como hace Sraffa- la razón-patrón en la ecuación en lugar de la tasa máxima de ganancia. Y es una contradicción porque el objeto de discusión es precisamente la problemática relación de las dos variables distributivas -salarios y ganancias- en el marco ide la producción conjunta! Al menos en ese marco establece Sraffa sus especulaciones sobre estas relaciones. No obstante, como casi tódo lo que hay de error o de inadecuado en PMpM, tiene solución con matices. En efecto y en este caso podemos tomar la tasa máxima de ganancia en (b.1), partir de la producción conjunta -es decir, partir de una matriz de productos finales $\boldsymbol{Y}$ con $\boldsymbol{n} \times \boldsymbol{n}$ elementos- y las ecuaciones, el gráfico y gran parte de la discusión siguen siendo válidos. El problema está en que ya no podemos garantizar un vector de precios positivos. Sin embargo y mientras el producto nacional PYI que aparece en la ecuación (b.4) sea positivo -y parece difícil lo contrario si hay excedente en gran parte de los bienes y servicios de la economía- todo lo dicho y las consecuencias del sistema de ecuaciones expuesto son válidas. Sraffa viene a sostener en este capítulo que, mientras en la producción simple existe siempre una relación inversa entre salarios y ganancias, en la producción conjunta esto no necesariamente es verdad. Si estuviéramos en la producción simple y con una economía productiva excedente en todas los bienes y servicios- se puede asegurar que esta relación inversa es cierta siempre que los empresarios no llevan las ganancias a unos niveles que hagan que la serie entre corchetes de (b.4) $\mathbf{l}+(\mathbf{1}+\mathbf{g}) \boldsymbol{A}+(\mathbf{1}+\mathbf{g})^{2} \boldsymbol{A}^{2}+\ldots+(\mathbf{l}+\mathbf{g})^{n-1} \boldsymbol{A}^{n-1}$ no sea convergente. Estas dudas de Sraffa sobre la posible no relación inversa entre salarios y ganancias no tiene mucha consistencia, porque el verdadero y casi único problema de la producción conjunta es la falta de garantía de que obtengamos en el modelo precios estrictamente positivos. Quizás Sraffa viera algo que no desarrolló o que le llenó de dudas y lo dejó como una conjetura.

\footnotetext{
86 No es fácil traducir en términos formales cuando Sraffa habla así de "en términos de la mercancía-patrón". Hay que entenderlo como que quiere utilizar esa construcción virtual como numerario, y el mejor candidato para ello es la razón-patrón $\mathbf{R}$, incluso aunque el modelo esté fuera de esta construcción matemática y se refiera a un modelo sobre la economía real.
} 


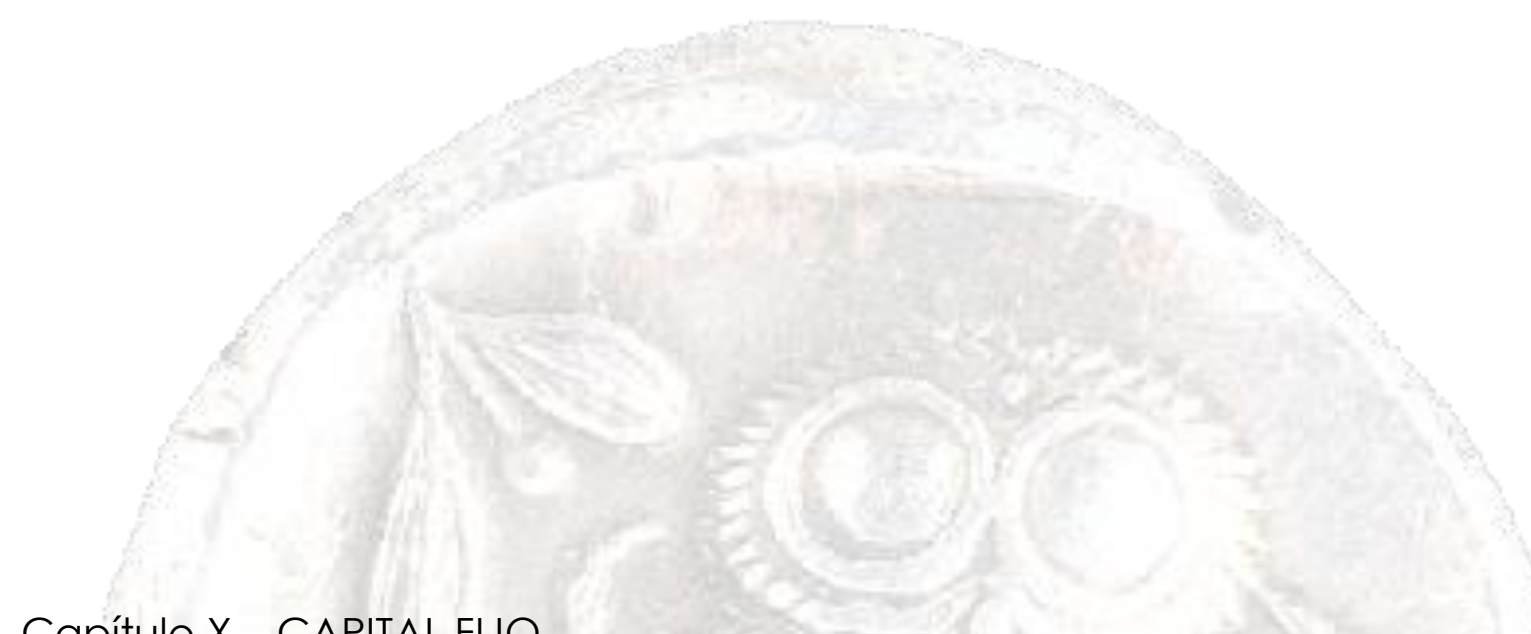

\section{Capítulo X - CAPITAL FIJO}

I - Conceptualmente este capítulo no debería tener apenas dificultad y, sin embargo, desde el punto de vista matemático es el más complicado y, por lo que sabemos, trajo de cabeza ${ }^{87}$ a Sraffa y al matemático Besicovitch, uno de los tres grandes matemáticos que le asesoraron. A esta dificultad formal añade Sraffa toda una serie de divagaciones sobre una supuesta "eficiencia constante", sin que lleguemos a distinguir entre la amortización financiera y la amortización física del capital fijo. La idea del capital fijo de la que parte Sraffa no es original -no podía serlo a pesar de lo original que son para la época sus planteamientos- porque, simplemente, se trata de incluir en su modelo aquellos bienes que son medios de producción y que no se consumen como tales en el mismo período de tiempo que el circulante. Circulante que es con lo que Sraffa -y nosotros siguiendo a Sraffa- trabaja en su libro hasta este momento. El modelo de Sraffa es el de un medio de producción de capital fijo que entra en la empresa como medio en un período, se corresponde con el capital fijo que sale como producto en el período siguiente. Lo que permanece igual, en cambio, es el circulante que se repite año tras año en la vida total del capital fijo y que está representado por-la ecuación $\mathbf{P Y}=(\mathbf{1 + g})[\mathbf{W L}+\mathbf{P X}]$. Sraffa concreta esa realidad y la incorpora a su modelo de la siguiente manera: "Este punto de vista implica que la misma máquina, a edades diferentes, debería ser tratada como otros tantos productos diferentes, cada uno con su propio precio" 88 . Se trata, además, de un caso especial de producción conjunta. Sraffa construye su modelo con supuestos muy restrictivos que iremos viendo a medida que

87 Kurz y Salvadori relatan las dificultades de Sraffa y la ayuda del matemático y amigo Besicovitch en el artículo Removing an insuperable obstacle in the way of an objetivist analisys: Sraffa attempts as fixed capital, 2008.

88 Epígrafe 74 del capítulo X de PMpM. 
desarrollamos su modelo. Partimos de la siguiente ecuación de definición del capital fijo de su término general:

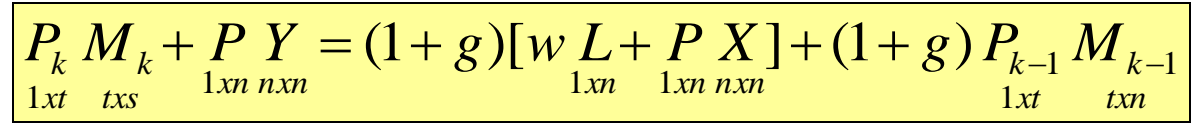

que en términos aritméticos es:

$$
p_{k} \sum_{h=1}^{s} M_{k h_{j}}+\sum_{i=1}^{n} p_{i} y_{i j}=(1+g)\left[w l_{j}+\sum_{i=1}^{n} p_{i} x_{i j}\right]+(1+g) p_{k-1} \sum_{h=1}^{s} M_{k-1, h j} \quad \forall j=1 \quad \text { a } n \quad \forall k=1 \quad a t
$$

donde $\boldsymbol{p}_{\boldsymbol{k}}$ es el valor89 del capital fijo del período $\boldsymbol{k}$ en la empresa al final de un

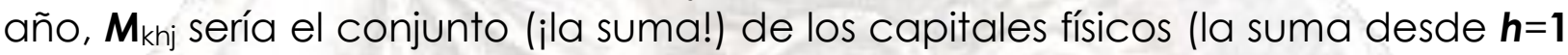
a $\boldsymbol{s}$ ) en el período $\boldsymbol{k}$ de vida útil asignados al proceso $\mathbf{j}$.

Llegado a este punto debemos detenernos un momento porque Sraffa dice que nuestra $\boldsymbol{M}_{\mathrm{khj}}$ sería "la cantidad de máquinas de un tipo dado que se requiere para producir anvalmente una cantidad de mercancía". Y la pregunta que podemos hacernos es: ¿̇cómo vamos a sumar distintos tipos de máquinas en términos físicos? Porque luego hay que multiplicarlas, además, por un supuesto único precio que es $\boldsymbol{p}_{\mathrm{k}}$ que es común a todos ellos 90 . ¿No habrá caído Sraffa en el mismo error que criticaba, con razón, a la economía marginalista Joan Robinson y el propio Sraffa? Podemos solventar en gran medida el problema porque cabe una generalización de lo anterior. En (10.2) se ha considerado que cada bien de capital físico $\boldsymbol{h}$ tiene un precio propio $\boldsymbol{p}_{\mathrm{kh}}$ correspondiente al período $\boldsymbol{k}$ de vida útil.

$$
\sum_{h=1}^{s} p_{k h} m_{k h j}+\sum_{i=1}^{n} p_{i} y_{i j}=(1+g)\left[w l_{j}+\sum_{i=1}^{n} p_{i} x_{i j}\right]+(1+g) \sum_{h=1}^{s} p_{k-1, h} m_{k-1, h, j} \quad \forall j=1 \quad \text { a } n \quad \forall k=1 \quad a t
$$

Vamos a utilizar indistintamente ambas interpretaciones, es decir, las dadas por las ecuaciones (10.1) y (10.2). Y, en términos matriciales, nos vale la (10.1) para ambas. Además, con esta formulación general dada por cualquiera de las dos ecuaciones anteriores, nos puede permitir hacer $\boldsymbol{h}=\mathbf{i}$ si queremos hacer corresponder -como parece que lo hace Sraffa en el texto mencionado- los capitales $\boldsymbol{h}$ (desde $\mathbf{1}$ a $\boldsymbol{s}$ ) de cualquier período $\boldsymbol{k}$ de vida útil (asignados a cualquiera de los procesos $\boldsymbol{j}$ ) con la producción de cada una de las

89 Equivalente al precio si estuviera listo para la venta. Como no lo está y no es ese su destino, empleamos el término de valor pero que, en el contexto que se hace, ha de entenderse que valor (como coste unitario) y precio son sinónimos.

90 Y no queda más remedio que así sea con el modelo de Sraffa. 
mercancías $\boldsymbol{i}$ del circulante. En ese caso la (10.3) $\boldsymbol{Y}^{-1} \boldsymbol{X}$-por ejemplo- se convertiría en:

$$
\sum_{i=1}^{n} p_{k i} m_{k i j}+\sum_{i=1}^{n} p_{i} y_{i j}=(1+g)\left[w l_{j}+\sum_{i=1}^{n} p_{i} x_{i j}\right]+(1+g) \sum_{i=1}^{n} p_{k-1, i} m_{k-1, i, j} \quad \forall j=1 \quad a \quad n \quad \forall k=1 \quad a \quad t
$$

No obstante preferimos seguir trabajando con la ecuación (10.3) como base de nuestra discusión e interpretación de Sraffa, porque eso nos permite mayor libertad en el desarrollo del modelo del capital fijo. La razón de ello es la de que no siempre se podrá asignar un medio de producción a un único bien o servicio producido. Pensemos en el ejemplo de una fábrica de cervezas que reparte a los comercios y que lleva varias marcas, varios tipos de cerveza (con alcohol, sin alcohol, tostada, etc.). El medio empleado sería el mismo (furgoneta o camión), pero los productos serían diversos.

Desarrollamos sin más dilación las ecuaciones que se corresponden a la vida útil del capital fijo en la empresa. En términos matriciales podemos usar la expresión $\boldsymbol{P}_{k} \boldsymbol{M}_{k}$ para todas las consideraciones anteriores, para todos los supuestos anteriores que implican distintos modelos del capital fijo, unos más cercanos a Sraffa y otros menos. Y decimos que nos vale porque lo decisivo será diferenciar los precios (el valor del capital físico) según la vida útil de la maquinaria, instalación, instrumentos, utillaje, etc. Así suponemos que la vida útil del capital tiene un período útil de $t$ años:

$$
\begin{aligned}
& P_{1} M_{1}+P Y=(1+g)[w L+P X]+(1+g) P_{0} M_{0} \\
& P_{2} M_{2}+P Y=(1+g)[w L+P X]+(1+g) P_{1} M_{1} \\
& P_{t} M_{t}+P Y=(1+g)[w L+P X]+(1+g) P_{t-1} M_{t-1}
\end{aligned}
$$

En las ecuaciones (10.1) hay que matizar que la $\boldsymbol{k}$ del sumatorio de $\mathbf{p}_{\mathrm{k}} \boldsymbol{m}_{\mathrm{kj}}$ del primer miembro de la igualdad (el capital fijo que sale como producto) va de 1 $a \boldsymbol{t}$, en cambio la $\boldsymbol{k}$ de la expresión correspondiente (el capital fijo que entra como medio) del segundo miembro va desde $\mathbf{0}$ a $\boldsymbol{t} \mathbf{t} \mathbf{1}$. Si ahora sumamos miembro a miembro todas las ecuaciones de (10.5) y eliminamos términos comunes, queda:

$$
p_{t} \sum_{h=1}^{s} m_{t h j}+t \sum_{i=1}^{n} p_{i} y_{i j}=t(1+g)\left[w l_{j}+\sum_{i=1}^{n} p_{i} x_{i j}\right]+p_{0} \sum_{h=1}^{s} m_{0 h j}+g \times\left\{\sum_{k=0}^{t-1} p_{k} \sum_{h=1}^{s} m_{k h j}\right\} \quad \forall j=1 \quad a \quad n
$$


O bien la alternativa preferente (más genérica) mencionada:

$$
\sum_{h=1}^{s} p_{t h} m_{t h j}+t \sum_{i=1}^{n} p_{i} y_{i j}=t(1+g)\left[w l_{j}+\sum_{i=1}^{n} p_{i} x_{i j}\right]+\sum_{h=1}^{s} p_{o h} M_{0 h j}+g \times\left\{\sum_{k=0}^{t-1} \sum_{h=1}^{s} p_{k h} m_{k h j}\right\} \quad \forall j=1 \quad a n
$$

Trabajaremos a partir de ahora con (10.7) en lugar de la (10.6), pero lo que digamos de la primera vale para esta segunda91. Recogemos el supuesto que llama Sraffa de "eficiencia constante", es decir supone que la tasa $f$ a la que se financia el capital fijo ${ }^{92}$ es constante, por lo que se cumple:

$$
\sum_{h=1}^{s} p_{k h} m_{k h j}=(1+f) \sum_{h=1}^{s} p_{k-1, h} m_{k-1, h, j}
$$

Y con este criterio pasamos de (10.7) a:

$$
\sum_{h=1}^{s} p_{t h} m_{t h j}+t \times \sum_{i=1}^{n} p_{i} y_{i j}=t(1+g)\left[w l_{j}+\sum_{i=1}^{n} p_{i} x_{i j}\right]+\sum_{h=1}^{s} p_{o h} m_{o h j}+g \times\left\{\sum_{k=o}^{t-1} \sum_{h=1}^{s}(1+f)^{k} p_{k h} m_{k h j}\right\} \quad \forall j=1 \quad a n
$$

Desarrollamos el último factor del segundo miembro y queda:

$$
\sum_{h=1}^{s} p_{t h} m_{t h j}+t \times \sum_{i=1}^{n} p_{i} y_{i j}=t(1+g)\left[w l_{j}+\sum_{i=1}^{n} p_{i} x_{i j}\right]+\sum_{h=1}^{s} p_{o h} m_{o h j}+g \times \frac{(1+f)^{t}-1}{f} \times \sum_{h=1}^{s} p_{o h} m_{o h j} \quad \forall j=1 \quad a n
$$

$$
\sum_{h=1}^{s} p_{t h} m_{t h j}+t \times \sum_{i=1}^{n} p_{i} y_{i j}=t(1+g)\left[w l_{j}+\sum_{i=1}^{n} p_{i} x_{i j}\right]+(1+g)^{t} \times \sum_{h=1}^{s} p_{o h} m_{o h j} \quad \forall j=1 \quad a \quad n
$$

91 Y también vale para el caso considerado de que $\mathbf{h}=\mathbf{i}$.

92 En contra de todas apariencia en una primera lectura de este capítulo, la "eficiencia constante" de la que habla Sraffa se refiere al cambio de valor financiero del capital fijo y no a su depreciación, que operaría en sentido contrario. Por otro lado habría que matizar que esta tasa financiera es una tasa monetaria que opera sobre ${\underline{\boldsymbol{P}_{k}}}_{\boldsymbol{M}_{k}}$ y no solo sobre $\boldsymbol{M}_{k}$. Sin embargo y, dado que los precios varían con el período $\mathbf{k}$ (a diferencia del capital circulante), podemos entender que la exigencia es solo sobre el capital físico. En la formulación interpretamos que lo que se deteriora es el valor de los bienes, es decir, el producto de precio por el bien, pero podemos entender que una de ellas sea fija y la otra variable o las dos variables. Lo que es necesario -para seguir en Sraffa- es que sobre el producto PM se opere lo que llama la "eficiencia constante". Matemáticamente, al menos, caben todas las posibilidades mencionadas. 
El sumando del sumatario de $\boldsymbol{p}_{\uparrow} \boldsymbol{m}_{\dagger}$ del primer miembro de (10.11) es un valor residual, que Sraffa considera que es equivalente a la chatarra y que carece de valor, por lo que la expresión de ese sumatorio $\boldsymbol{p}_{+} \boldsymbol{m}_{+}=\mathbf{0}$. Y dividiendo ahora la ecuación (10.11) entre el número de sumandos $t$, puesto que (10.11) es el resultado de haber sumado todas las ecuaciones de (10.5), queda:

$$
\sum_{i=1}^{n} p_{i} y_{i j}=(1+g)\left[w l_{j}+\sum_{i=1}^{n} p_{i} x_{i j}\right]+\frac{(1+g)^{t}}{t} \times \sum_{h=1}^{s} p_{o h} m_{o h j} \quad \forall j=1 \quad a \quad n
$$

Lo sorprendente, prima facie, de (10.12) es que no es la expresión matemática sobre el capital fijo a la que llega Sraffa, que es:

$$
\sum_{i=1}^{n} p_{i} y_{i j}=(1+g)\left[w l_{j}+\sum_{i=1}^{n} p_{i} x_{i j}\right]+\frac{g(1+g)^{t}}{(1+g)^{t}-1} \times \sum_{h=1}^{s} p_{o h} m_{o h j} \quad \forall j=1 \quad a \quad n
$$

Ahora bien, para que (10.12) y (10.13) fueran iguales debería ocurrir que se igualaran a su vez las expresiones $\mathbf{g} /\left[(\mathbf{1}+\mathbf{g})^{\dagger}-\mathbf{1}\right]=\mathbf{1} / \mathbf{t}$, lo cual daría que $\mathbf{1 + g} \mathbf{t}=(\mathbf{1}+\mathbf{g})^{\dagger}$, y que, como todo matemático sabe, es una expresión aproximadamente igual si la tasa $\mathbf{g}$ es pequeña y el número de términos $\boldsymbol{s}$ también es pequeño. También puede entenderse que el dar el valor cero a lo que Sraffa llama chatarra pueda compensar este desliz matemático.

Recapitulemos los supuestos que hemos hecho hasta llegar a (10.13). Hemos partido desde luego con tasas unitarias de salarios $\boldsymbol{w}$ y tasas de ganancia $\mathbf{g}$, pero esto es subsanable como hemos visto con la ecuación (7.3) del capítulo anterior. Hemos supuesto que las tasas a de amortización del capital fijo son iguales entre sí e iguales a las tasas de ganancia $\mathbf{g}$ del modelo, y que el valor de la chatarra es cero. Al final hemos constado que no son enteramente equivalentes el modelo desarrollado aquí y el que incorpora Sraffa en su modelo. La diferencia está entre $\mathbf{1 + g t}$ y $(\mathbf{1}+\mathbf{g})^{\mathbf{t}}$. La conclusión es que no se pueden hacer el valor residual (chatarra) cero y, simultáneamente, que la tasa de ganancia $\mathbf{g}$ igual a la tasa de depreciación a. El problema de todo lo anterior es que nos hemos alejado de la solución formal que da Sraffa y, también, conceptual, porque el italiano considera que eficiencia constante es la "carga anual a pagar por intereses y żdepreciación?"... y "esta carga anual será igual a una anualidad fija..."93. Pero si la depreciación de Sraffa coincide con la parte de la anualidad correspondiente al principal financiero lo que ocurre es que el valor del circulante PY se incrementa $-y$, por tanto, el precioen lugar de rebajarse, con lo cual el valor de la chatarra $\boldsymbol{P}_{+} \boldsymbol{M}_{\dagger}$ (valor final del capital fijo) se incrementaría. Por ello no queda más remedio para seguir el 
texto de Sraffa -y no simplemente sus ecuaciones- añadir a la carga anual financiera soportada por el capital fijo año a año una depreciación física o, al menos, en términos de valor, del capital fijo, tal que ese valor en un período cualquiera de su vida útil $\boldsymbol{P}_{\mathrm{k}} \boldsymbol{M}_{\mathrm{k}}$ jsea menor! que el valor de ese mismo capital o medio de producción en el período anterior. Por eso decimos que valor residual cero (chatarra) y eficiencia constante entendida como anvalidad soportada año tras año no se corresponden. No obstante vamos a demostrar en el epígrafe siguiente cómo llegar a la formulación de Sraffa.

II - La mejor forma de llegar a la formulación de Sraffa es mediante sustituciones sucesivas de la ecuación fundamental del capital fijo:

$$
\sum_{h=1}^{s} p_{k h} m_{k h j}+\sum_{i=1}^{n} p_{i} y_{i j}=(1+g)\left[w l_{j}+\sum_{i=1}^{n} p_{i} x_{i j}\right]+(1+g) \times \sum_{h=1}^{s} p_{k-1, h} m_{k-1, h, j} \quad \forall k=1 \text { at } \quad \forall j=1 \text { a } n
$$

Que en términos matriciales sería:

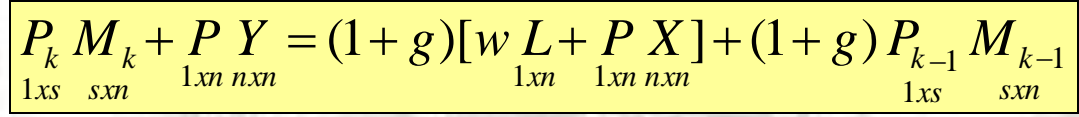

Ya hemos desarrollado el conjunto de estas ecuaciones en el primer epígrafe

$$
\begin{aligned}
& P_{1} M_{1}+P Y=(1+g)[w L+P X]+(1+g) P_{0} M_{0} \\
& P_{2} M_{2}+P Y=(1+g)[w L+P X]+(1+g) P_{1} M_{1}
\end{aligned}
$$

$$
P_{t} M_{t}+P Y=(1+g)[w L+P X]+(1+g) P_{t-1} M_{t-1}
$$

Lo que nos dice Sraffa -o así hay que interpretarlo- en epígrafe 79 es que va a ir sustituyendo $\boldsymbol{P}_{1} \boldsymbol{M}_{1}$ de la primera ecuación matricial en la segunda, hecho esto, se hará lo mismo con $\boldsymbol{P}_{2} \boldsymbol{M}_{2}$ en la tercera, y así sucesivamente hasta la penúltima, sustituyendo entonces $\boldsymbol{P}_{\mathbf{t}_{-1}-\boldsymbol{M}_{\mathrm{t}-1}}$ en la última. El resultado es:

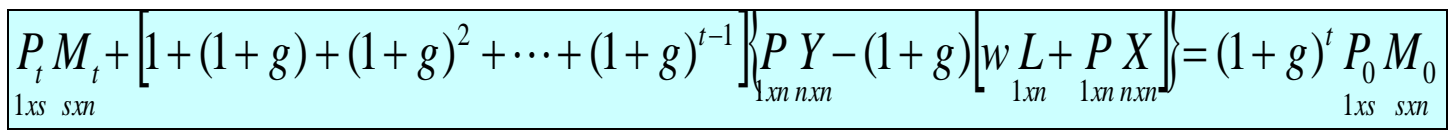

Y, efectuando la suma de la primera expresión entre corchetes del primer miembro de la ecuación, sale: 


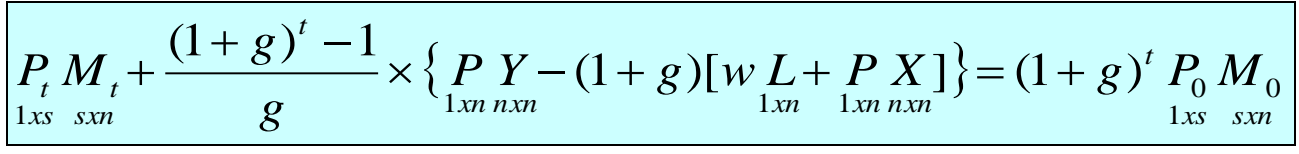

Y tras manipulaciones elementales aritméticas:

$$
\frac{g}{(1+g)^{t}-1} \times \underset{1 x s}{P_{t}} M_{t x n}+\underset{1 \times n n x n}{P}=(1+g)[w \underset{1 \times n}{L}+\underset{1 x n n x n}{P} \underset{X}{X}]+\frac{g(1+g)^{t}}{(1+g)^{t}-1} \times \underset{1 \times s}{P_{x x n}} M_{0}
$$

Sraffa considera que $\boldsymbol{P}_{\uparrow} \mathbf{M}_{\uparrow}$ es la chatarra al que se le puede dar el valor cero, con lo que llega a la ecuación:

$$
\underset{1 x n \text { nxn }}{P}=(1+g)[w \underset{1 \times n}{L}+\underset{1 \times n}{P} \underset{n \times n}{X}]+\frac{g(1+g)^{t}}{(1+g)^{t}-1} \times \underset{1 x s}{P_{s x n}} M_{0}
$$

que es la de Sraffa del epígrafe 76. ¿Y qué ha pasado con la "eficiencia constante" como método de amortización de la que nos habla Sraffa? Pues sencillamente que nos hemos olvidado de ella porque, si la tuviéramos en cuenta, no sale la flamante ecuación de Sraffa de ese epígrafe. Salvo que pensemos que "eficiencia constante" no se refiere a una tasa de amortización del capital fijo, sino a la constancia del circulante a lo largo de los $t$ períodos de vida útil del capital fijo. O para decirlo con las palabras de Sraffa en ese epígrafe "Las cantidades de medios de producción, de trabajo, y del producto principal son iguales en los varios procesos, de acuerdo con el supuesto de eficiencia constante durante la vida de la máquina". Sin embargo en el epígrafe $\mathbf{7 8}$ dice que "si la máquina tuviera la misma vida de trabajo y eficiencia constante en todas las industrias, los valores contables de cada edad serían iguales en todas ellas". Aquí, por tanto, ya no se refiere al hecho de la constancia del circulante -como nos ha dicho en el epígrafe 76-, sino a la financiación del capital fijo. Para liarla más, Sraffa nos dice en el epígrafe $\mathbf{8 1}$ que "supongamos que un tractor requiera, directa e indirectamente, 4 unidades de trabajo para ser producido y que tiene una vida de 4 años con eficiencia constante" y a continuación nos habla de cómo va disminuyendo la unidades de trabajo que son necesarias "para ser producida", hasta que llega al final de su vida útil (se convierte en chatarra). Todos estos comentarios de Sraffa abogan por considerar que, en contra de una primera lectura de este capítulo de su obra, de que no presenta ningún esquema de depreciación física del capital fijo94. Ni siquiera podemos asegurar que lo tenga en términos de valor puesto que no nos asegura que los precios vayan a permanecer constantes a lo largo de la vida útil de la maquinaria, instalaciones, utillaje, etc. En el epígrafe $\mathbf{7 6}$ parece inclinarse por ese supuesto, pero entonces, si no hay depreciación física, lo que hace Sraffa es una mera amortización financiera

$94 \mathrm{Ni}$ siquiera en términos de valor, es decir, para $\mathbf{P}_{\mathrm{k}} \mathbf{M}_{\mathrm{k}}$. 
mediante el cálculo de la anualidad empleada por los capitales financieros. De ahí que emplee la tasa de ganancia $\mathbf{g}$ para la supuesta amortización del capital fijo en lugar de una tasa de depreciación física.

Todas estas consideraciones podrían parecer carencias o incoherencias de este capítulo de Sraffa pero, sea cual sea la interpretación que le demos, nos permite desarrollos alternativos de su teoría del capital fijo, incorporando tanto una amortización meramente financiera como una amortización por depreciación física de los instrumentos de producción\%5. Algunos de los epígrafes que se exponen a continuación representan distintos modelos de depreciación física del modelo de Sraffa.

III - Para terminar el cuerpo principal de este capítulo de la teoría del capital fijo de Sraffa o desarrollado a partir de Sraffa, vamos a generalizar en lo posible hasta obtener una ecuación que no adolezca de algunas de las limitaciones de la ecuaciones representativas que dio y hemos discutido el gran economista italiano. En primer lugar vamos a generalizar las tasas de ganancia $\boldsymbol{B}$ y de salarios $\boldsymbol{W}$ mediante matrices $\boldsymbol{n} \times \boldsymbol{n}$ que puedan tener $\boldsymbol{n}$ elementos si son diagonales, o bien, también $\mathbf{n} \times \boldsymbol{n}$ elementos si no lo son. Además vamos a diferenciar los bienes y servicios de consumo -que van a estar representados por la matriz $\boldsymbol{C}$ - de la de bienes y servicios finales $\boldsymbol{Y}$ que son a su vez medios de producción, pero de duración máxima del período convencional que se estima que es el circulante. Estos cambios siguen sin ser meras descripciones de la realidad, es decir, sin caer en un mero empirismo sin capacidad explicativa, pero solventan parcialmente las limitaciones del modelo de Sraffa. Una ecuación tal (10.22) vendría dada por:

$\sum_{h=1}^{s} p_{m k h} m_{k h j}+\sum_{l=1}^{z} p_{c l} C_{l j}+\sum_{i=1}^{n} p_{i} y_{i j}=\sum_{i=1}^{n} l_{i}\left[w_{i j}\left(1+b_{i j}\right)\right]+\sum_{i=1}^{n} p_{i}\left[x_{i j}\left(1+b_{i j}\right)\right]+\sum_{h=1}^{s} p_{m k-1, h}\left[m_{k-1, h, j}\left(1+b_{m k-1 h j}\right)\right]$

Y lo anterior para todo $\boldsymbol{k}$ desde $\mathbf{1}$ a $\boldsymbol{t}$ y para todo $\boldsymbol{j}$ de $\mathbf{1}$ a $\boldsymbol{n}$. También en la anterior los productos $\boldsymbol{w}_{\mathrm{ij}}\left(\mathbf{1}+\boldsymbol{b}_{\mathrm{ij}}\right), \mathrm{x}_{\mathrm{ij}}\left(\mathbf{1}+\boldsymbol{b}_{\mathrm{ij}}\right)$ y $\boldsymbol{m}_{\mathrm{k}-1, \mathrm{~h}, \mathrm{j}}\left(\mathbf{1}+\boldsymbol{b}_{\mathrm{mhj}}\right)$ han de entenderse como productos aritméticos, productos que se entenderán mejor ahora con la ecuación matricial correspondiente a (10.22):

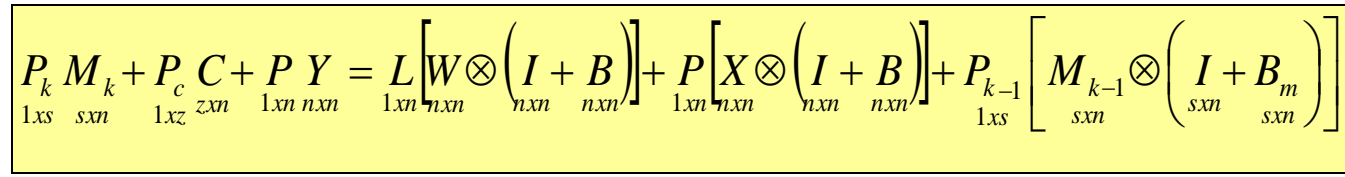

\footnotetext{
95 Si no incorporamos una depreciación física de los instrumentos de producción de vida superior a un año (período convencional) malamente podremos considerar que esos instrumentos se han achatarrado y que su valor es cero.
} 
En (10.23) los productos entre matrices $\boldsymbol{W}$ y $(\mathbf{I}+\mathbf{B}), \boldsymbol{X}$ y $(\mathbf{I}+\mathbf{B}), \boldsymbol{M}_{k-1}$ y $\left(\mathbf{I}+\boldsymbol{B}_{m}\right)$ son productos aritméticos de los elementos $\boldsymbol{w}_{\mathrm{ij}}, \mathbf{x}_{\mathbf{i j}}$ y $\boldsymbol{m}_{\mathrm{k}-1, \mathrm{~h}, \mathrm{j}}$ que se multiplican aritméticamente (elemento a elemento) por los elementos dados por las sumas $\mathbf{1}+\boldsymbol{b}_{i j}$ y $\mathbf{1}+\boldsymbol{b}_{m, k-1, h, j}$ correspondientes. En cambio, los productos resultantes entre corchetes de la segunda parte de la igualdad sí son productos matriciales (matrices post-multiplicadas) respecto a los vectores $\boldsymbol{L}, \boldsymbol{P}$ y $\boldsymbol{P}_{\mathrm{k}-1}$ (los resultantes son todos vectores $1 \times n$ ). Señalar que, en este caso de generalización, la matriz de ganancias $\mathbf{B}$ que conlleva el circulante no es la misma que la matriz $\boldsymbol{B}_{\mathrm{m}}$ del capital fijo, salvo en el caso particular de tasas unitarias, que sería el caso particular con el que trabaja Sraffa permanentemente. La matriz $\mathbf{C}$ de bienes de consumo tampoco tiene que ser cuadrada (to natural es lo que no lo sea) porque no tiene por qué coincidir el número de procesos $\boldsymbol{n}$ (que afectan por igual al circulante como al fijo) con el total de bienes y servicios $\mathbf{z}$ consumidos ${ }^{96}$. También -en aras de la generalidad- hemos hecho que las ganancias del capital fijo puedan ser variables de año en año, es decir, hemos utilizado como

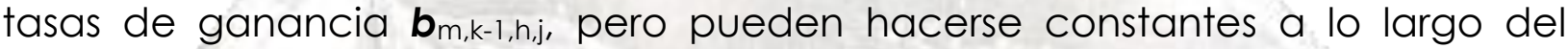
ciclo, de tal forma que $\boldsymbol{b}_{\mathrm{m} 1 \mathrm{hj}}=\boldsymbol{b}_{\mathrm{m} 2 \mathrm{hj}}=\ldots=\boldsymbol{b}_{\mathrm{mthj}}$.

IV - Yolviendo al cuerpo principal de este capítulo, a pesar de de nuestros esfuerzos no hemos dado una solución satisfactoria a la teoría de la amortización financiera (que no física) que nos legó Sraffa precisamente por tratar de reconstruir el capítulo sobre el capital fijo de $\mathbf{P M p M}$. Ahora nos vamos a centrar en dar varias interpretaciones de lo que llamó Sraffa "eficiencia constante" (epígrafe $\mathbf{7 7}$ de $\mathbf{P M p M}$ ), pero haciendo implícitos algunos posibles modelos de depreciación física. Recordemos que no parecen compatibles dar un valor cero al valor residual del capital fijo (chatarra) y, simultáneamente, igualar tasa de ganancia con tasa de amortización (eficiencia constante tal como la llama Sraffa). Por eso vamos a dar varias interpretaciones al posible sistema de amortización que el italiano nos presenta en el epígrafe $\mathbf{7 7 .}$

IV.a - En el primero de ello vamos a suponer que el capital fijo del período $\boldsymbol{k}$ se relaciona con el capital fijo del período $\boldsymbol{k}-\mathbf{1}$ mediante la ecuación:

$$
P_{k} M_{k}=(1-a) P_{k-1} M_{k-1}
$$

Con ello queremos señalar que el valor del capital fijo de un período es fruto de un descuento (depreciación ${ }^{97}$ ) del capital fijo del período anterior. Y a es la

96 Esta discusión la lleva Sraffa en los inicios del capítulo VIl sobre la Producción conjunta, aunque nunca distinguió formalmente (con matrices diferentes) los bienes de consumo de los bienes finales de capital fijo. Por ello se vio obligado a hacer iguales el número de procesos de producción y los bienes y servicios (mercancías en Sraffa) producidos. Es, desde luego, un defecto que puede ser subsanado, pero que tienen sus consecuencias a la hora de recontar los grados de libertad y sobre la posibilidad o no de que el modelo sea determinístico o no en el cálculo de los precios.

97 Que puede entenderse en términos físicos (lo cual afectaría a $\boldsymbol{M}_{k}$ ), o en términos de valor, lo cual afectaría al producto $\mathbf{P}_{\mathbf{k}} \mathbf{M}_{\mathrm{k}}$. Las ecuaciones de (a.2) que siguen sirven para ambos criterios. 
tasa de descuento o amortización (con $\mathbf{0}<\mathbf{a}<\mathbf{1}$ ). Vamos a seguir el mismo proceso que en el epígrafe del cuerpo principal de este capítulo y presentamos las mismas ecuaciones que Sraffa presenta a lo largo de la vida útil del capital fijo tal como:

$$
\begin{aligned}
& P_{1} M_{1}+P Y=(1+g)[w L+P X]+(1+g) P_{0} M_{0} \\
& P_{2} M_{2}+P Y=(1+g)[w L+P X]+(1+g) P_{1} M_{1} \\
& P_{t} M_{t}+P Y=(1+g)[w L+P X]+(1+g) P_{t-1} M_{t-1}
\end{aligned}
$$

Al sumar miembro a miembro el conjunto de ecuaciones matriciales anteriores, eliminar términos comunes queda, pasar los costes del circulante $(\mathbf{1}+\mathbf{g})[\mathbf{w L}+\boldsymbol{P X}]$ al primer miembro, sale:

$$
\sum_{h=1}^{s} p_{t h} m_{t h j}+t\{P Y-(1+g)[w L+P X]\}=\sum_{h=1}^{s} p_{o h} m_{o h j}+g \sum_{k=0}^{t-1} \sum_{h=1}^{s} p_{k h} m_{k h j} \quad \forall j=1 a n
$$

Ahora vamos calculando los sucesivos valores de $\boldsymbol{P}_{k} \boldsymbol{M}_{\mathrm{k}}$ de acuerdo con (10.24) y los sustituimos en (10.26) y nos sale la ecuación en términos matriciales:

$$
(1-a)^{t} \times P_{0} M_{0}+t\{P Y-(1+g)[w L+P X]\}=P_{0} M_{0}+g \sum_{k=0}^{t-1}(1-a)^{k} P_{0} M_{0}
$$

Y desarrollada la serie que hay en el lado derecho de la ecuación obtenemos:

$$
(1-a)^{t} \times P_{0} M_{0}+t\{P Y-(1+g)[w L+P X]\}=P_{0} M_{0}+\frac{g\left[1-(1-a)^{t}\right]}{a t} \times P_{0} M_{0}
$$

Tras manipulaciones algebraicas elementales sale definitivamente:

$$
P Y=(1+g)[w L+P X]+\frac{(a+g)\left[1-(1-a)^{t}\right]}{a t} \times P_{0} M_{0}
$$

Y en el caso que sigamos el criterio de Sraffa y hagamos que la tasa de depreciación física a coincida con la tasa de ganancia, quedaría: 
(10.30)

$P Y=(1+g)[w L+P X]+\frac{2\left[1-(1-a)^{t}\right]}{t} \times P_{0} M_{0}$

Y los precios de los bienes del circulante P serían:

(10.30)

$$
P Y=\left\{(1+g) w L+\frac{2\left[1-(1-a)^{t}\right]}{t} \times P_{0} M_{0}\right\}[Y-(1+g) X]^{-1}
$$

IV.b - Otra posible interpretación de ese epígrafe relacionaría el capital fijo del período $\boldsymbol{k}$ respecto a la anterior $\boldsymbol{k}-\mathbf{1}$ tal como:

$$
P_{k} M_{k}=\frac{1}{1+b} \times P_{k-1} M_{k-1}
$$

Y suponemos que $\mathbf{b}>\mathbf{1}$. Partimos de la ecuación general a todos los sistemas iniciados con Sraffa:

$$
P_{t} M_{t}+t\{P Y-(1+g)[w L+P X]\}=P_{0} M_{0}+g \sum_{k=0}^{t-1} P_{k} M_{k}
$$

Si hacemos lo mismo que con el sistema anterior de amortización obtenemos la ecuación intermedia:

$$
\frac{1}{(1+b)^{t}} \times P_{0} M_{0}+t P Y-t(1+g)[w L+P X]=P_{0} M_{0}+\frac{g(1+b)\left[(1+b)^{t}-1\right]}{b(1+b)^{t}} \times P_{0} M_{0}
$$

Y de esta llegamos a la definitiva:

$$
P Y=(1+g)[w L+P X]+\frac{\left[(1+b)^{t}-1\right][b+g(1+b)]}{t b(1+b)^{t}} \times P_{0} M_{0}
$$

Y a (10.34) se llegaría de igual forma si se hubiera sustituido en (10.31) (1-a) por $(\mathbf{1 + b})^{-1}$. Aquí podemos igualar tasa de ganancia $\mathbf{g}$ con una supuesta tasa de amortización, puesto que lo que hay es un factor $\mathbf{1} /(\mathbf{1 + b})$ que relaciona el valor 
del capital fijo con el mismo capital en términos físicos en un período anterior. Tampoco podemos hacer cero el valor residual puesto que ello solo sería posible si desde el principio -es decir, desde $\boldsymbol{P}_{0} \boldsymbol{M}_{0}-$ el valor de este capital hubiera sido cero, lo cual no tiene sentido. Como ya hemos comentado, se ve con estos dos sistemas de depreciación del capital fijo no puede hacerse cero el valor residual si se quiere mantener un sistema de "eficiencia constante" de amortización. Eficiencia constante y valor residual cero son incompatibles; también lo son valor residual cero e igualar tasas de ganancia y de amortización. Solo en algunos sistemas se pueden igualar la tasa de ganancia y de amortización pero sin hacer cero el valor residual. Dicho de otra manera, la única manera de llegar al modelo de Sraffa es abandonar su propio criterio de amortización y considerar que las amortizaciones de un período no dependen funcionalmente -matemáticamente- de un período anterior. Solo entonces se pude recurrir al criterio económico de considerar iguales tasas de ganancia y de amortización. Otra cosa es que este criterio carezca o no de sentido. Eso, en este trabajo, no lo discutimos puesto que aquí nos limitamos a los aspectos matemáticos: su escudriñamiento, la coherencia interna o no de los formalismos y algunos posibles desarrollos.

IV.c - Inspirándonos en Sraffa podemos damos un tercer mecanismo de amortización del capital fijo. Dice Sraffa en el epígrafe $\mathbf{8 0}$ que "si suponemos que el tipo de beneficio es cero, el valor de tal máquina descenderá a pasos iguales de $1 / \mathbf{n}$ del valor original en cada uno de los años". Supongamos que no exigimos como Sraffa que el tipo de beneficio sea cero. El mecanismo de amortización del capital fijo del período $\boldsymbol{k}$ en relación al anterior $\mathbf{k}-\mathbf{1}$ estaría dado por la formula:

$$
P_{k} M_{k}=\frac{t-1}{t} \times P_{k-1} M_{k-1}
$$

Siendo la amortización del período $\boldsymbol{k}$ la dada por (1/t) $\boldsymbol{P}_{k-1} \boldsymbol{M}_{k-1}$. Para mayor comodidad llamaremos $\mathbf{b}$ a $(\boldsymbol{t}-\mathbf{1}) / \boldsymbol{t}$, con lo que (10.35) queda:

$$
P_{k} M_{k}=b \times P_{k-1} M_{k-1}
$$

Si ahora procedemos a las sustituciones sucesivas desde $\mathbf{k}-\mathbf{1}=\mathbf{0}$ a $\boldsymbol{k}=\boldsymbol{t}$ tenemos que:

$$
P_{t} M_{t}=b^{t} \times P_{0} M_{0}
$$


Traemos aquí la ecuación general de Sraffa del capital fijo (la podríamos tildar con tal nombre porque permanece constante sea cual sea el sistema de amortización del capita fijo):

$$
P_{t} M_{t}+t\{P Y-(1+g)[w L+P X]\}=P_{0} M_{0}+g \sum_{k=0}^{t-1} P_{k} M_{k}
$$

La formulación anterior, con el sumatorio aplicándolo al criterio de (10.37) daría:

$$
P_{t} M_{t}+t\{P Y-(1+g)[w L+P X]\}=P_{0} M_{0}+g \sum_{k=0}^{t-1} b^{k} P_{k} M_{k}
$$

Y el resultado de ejecutar el sumatorio de (10.39), más el resultado de $\boldsymbol{P}_{\uparrow} \mathbf{M}_{\uparrow}$ de acuerdo con (10.37) y traspasado el término al otro lado de la igualdad es:

$$
t\{P Y-(1+g)[w L+P X]\}=P_{0} M_{0}+\frac{g\left(1-b^{t}\right)}{1-b} \times P_{0} M_{0}-b^{t} P_{0} M_{0}
$$

Y tras maniobras algebraicas elementales queda:

$$
P Y=(1+g)[w L+P X]+\frac{\left(1-b^{t}\right)[g+1-b]}{t(1-b)} \times P_{0} M_{0}
$$

Y sustituyendo $\mathbf{b}$ por su valor, es decir, por $(\boldsymbol{t}-\mathbf{1}) / \mathbf{t}$ queda:

$$
P Y=(1+g)[w L+P X]+\frac{(1+g t)\left[t^{t}-(t-1)^{t}\right]}{t^{t+1}} \times P_{0} M_{0}
$$

Que en términos aritméticos es:

$$
\sum_{i=1}^{n} p_{i} y_{i j}=(1+g)\left[w l_{j}+\sum_{i=1}^{n} p_{i} x_{i j}\right]+\frac{(1+g t)\left[t^{t}-(t-1)^{t}\right]}{t^{t+1}} \times \sum_{h=1}^{s} p_{o h} m_{o h j} \quad \forall j=1 \quad a \quad n
$$


IV.d - Los dos epígrafes que siguen quizás se aparten algo más de Sraffa si leemos al economista italiano entre líneas, pero no si lo hacemos literalmente. En el epígrafe $\mathbf{7 5}$ de su obra capital nos dice que "Supongamos que una máquina que trabaja con eficiencia constante a lo largo de su vida. La carga anval a pagar por intereses y depreciación respecto de ella debe ser constante". Es verdad que luego habla de "anualidad", lo cual remite al cálculo financiero (en concreto a la actualización de rendimientos futuros a tasa constante), pero quedémonos con la parte subrayada y veamos un posible sistema de amortización en este epígrafe y en el siguiente uno similar. Partimos -como ya hemos expuesto- de la ecuación fundamental del capital fijo de Sraffa que es común a todos los sistemas amortización puesto que, precisamente, aún no define ninguno de ellos.

$$
P_{t} M_{t}+t\{P Y-(1+g)[w L+P X]\}=P_{0} M_{0}+g \sum_{k=0}^{t-1} P_{k} M_{k}
$$

Es decir, en la ecuación anterior ya hemos sumado todas las ecuaciones de la vida útil del capital y eliminados términos comunes a partir de la ecuación de definición del capital fijo:

$$
P_{k} M_{k}+P Y=(1+g)[w L+P X]+(1+g) P_{k-1} M_{k-1}
$$

Pues bien, inspirándonos en el párrafo mencionado del texto de Sraffa vamos a suponer un sistema de amortización en el que la carga anual se reparta todos los años de la vida útil en proporción a los números naturales. Es decir, el primer año se amortiza el capital fijo inicial (nuestro $\boldsymbol{P}_{0} \mathbf{M}_{0}$ ) dividiendo este capital entre el número de años $\boldsymbol{t}$ de vida útil, el segundo entre $\mathbf{2 t} \boldsymbol{t}$, y así sucesivamente, hasta el último año en el que no hay carga de amortización puesto que ya se ha liquidado el valor residual (que en este caso coincidiría con el valor cero de Sraffa de la chatarra). Exponemos a continuación cuál sería el valor del capital fijo $\boldsymbol{P}_{k} \boldsymbol{M}_{k}$ de un año cualquiera en relación con su valor original $\boldsymbol{P}_{0} \mathbf{M}_{0}$ :

$$
\begin{aligned}
& P_{1} M_{1}=\left(1-\frac{1}{t}\right) P_{0} M_{0} \\
& P_{2} M_{2}=\left(1-\frac{2}{t}\right) P_{0} M_{0} \\
& P_{t-1} M_{t-1}=\left(1-\frac{t-1}{t}\right) P_{0} M_{0}
\end{aligned}
$$


$P_{t} M_{t}=\left(1-\frac{t}{t}\right) P_{0} M_{0}=0$

Si ahora sustituimos estos sucesivos valores en la ecuación fundamental (10.44) queda:

$$
P_{t} M_{t}+t\{P Y-(1+g)[w L+P X]\}=P_{0} M_{0}+g\left\{P_{0} M_{0}+P_{1} M_{1}+\cdots P_{t-1} M_{t-1}\right\}
$$

$$
P_{t} M_{t}+t\{P Y-(1+g)[w L+P X]\}=P_{0} M_{0}+g\left\{1+\left(1-\frac{1}{t}\right)+\left(1-\frac{2}{t}\right)+\cdots+\left(1-\frac{t-1}{t}\right)\right\} P_{0} M_{0}
$$

Ya hemos visto en (10.46) que, con este sistema de amortización, el valor residual del capital fijo $\boldsymbol{P}_{\uparrow} \mathbf{M}_{\dagger}$ necesariamente vale cero. Con esto dividimos toda la ecuación entre $\boldsymbol{t}$ (vida útil del capital fijo), manipulamos algo la ecuación anterior y sale:

$$
P Y-(1+g)[w L+P X]=\frac{1}{t} P_{0} M_{0}+\frac{1}{t} \times \frac{g}{t}\{(t-0)+(t-1)+\cdots+2+1\} P_{0} M_{0}
$$

Y el sumatorio que hay entre corchetes en el lado derecho es la suma de los términos de una progresión aritmética cuyo primer valor es 1 , el último es $\boldsymbol{t}$ y el número de términos es también $\boldsymbol{t}$, por lo que (10.49) queda finalmente:

$$
P Y=(1+g)[w L+P X]+\frac{1}{t} P_{0} M_{0}+\frac{1}{t} \times \frac{g}{t} \times \frac{(1+t) t}{2} \times P_{0} M_{0}
$$

Y que en términos aritméticos, tras reducir términos, es:

$$
\sum_{i=1}^{n} p_{i} y_{i j}=(1+g)\left[w l_{j}+\sum_{i=1}^{n} p_{i} x_{i j}\right]+\left\{\frac{1}{t}+\frac{g(1+t)}{2 t}\right\} \times \sum_{h=1}^{s} p_{o h} m_{o h j} \quad \forall j=1 \quad a n
$$

En la anterior el valor de la amortización solo física (es decir, con $\mathbf{g = 0}$ ) del capital fijo sería $(\mathbf{1} / \mathbf{t}) \mathbf{P}_{0} \mathbf{M}_{0}$, es decir, se repartiría la amortización anual proporcionalmente a los años de vida del capital. 
IV.e - Podemos generalizar el modelo de depreciación de IV.a estableciendo que las tasas de amortización física del capital (los medios de producción) no presentan un tipo constante a lo largo de la vida útil de los equipos, de tal forma que para el primer período de amortización es una tasa $\boldsymbol{a}_{1}$, para el segundo $\boldsymbol{a}_{2}$, y así sucesivamente hasta para el período último $\boldsymbol{t}$, que presentaría la tasa $\boldsymbol{a}_{\mathrm{t}}$. Esto se traduciría en que el capital físico de cada período $\boldsymbol{P}_{\mathrm{k}} \boldsymbol{M}_{\mathrm{k}}$ estaría en relación con el anterior $\boldsymbol{P}_{\mathrm{k}-1} \boldsymbol{M}_{\mathrm{k}-1}$ y en relación con el original $\boldsymbol{P}_{0} \mathbf{M}_{0}$ de la siguiente manera:

$$
\begin{aligned}
& P_{1} M_{1}=\left(1-a_{1}\right) P_{0} M_{0} \\
& P_{2} M_{2}=\left(1-a_{2}\right) P_{1} M_{1}=\left(1-a_{1}\right)\left(1-a_{2}\right) P_{0} M_{0} \\
& P_{t} M_{t}=\left(1-a_{t}\right) P_{t-1} M_{t-1}=\left(1-a_{1}\right)\left(1-a_{2}\right) \ldots\left(1-a_{t}\right) P_{0} M_{0}
\end{aligned}
$$

Y si ahora traemos la ecuación fundamental de la amortización financiera (solo) de Sraffa:

$$
P_{t} M_{t}+t\{P Y-(1+g)[w L+P X]\}=P_{0} M_{0}+g \sum_{k=0}^{t-1} P_{k} M_{k}
$$

Y llamamos $\boldsymbol{P}_{0} \mathbf{M}_{\mathbf{0}}=\mathbf{1}, \boldsymbol{P}_{1} \mathbf{M}_{\mathbf{1}}=\left(\mathbf{1}-\boldsymbol{a}_{1}\right) \boldsymbol{P}_{0} \mathbf{M}_{\mathbf{0}}, \boldsymbol{P}_{2} \mathbf{M}_{2}=\left(\mathbf{1}-\boldsymbol{a}_{1}\right)\left(\mathbf{1}-\boldsymbol{a}_{2}\right) \boldsymbol{P}_{0} \mathbf{M}_{\mathbf{0}}$, y el último término, $P_{t} M_{t}=\left(1-a_{1}\right) \ldots\left(1-a_{t}\right) P_{0} M_{0}$, y podemos escribir la ecuación:

$$
\prod_{k=1}^{k=t}\left(1-a_{k}\right) P_{0} M_{0}+t\{P Y-(1+g)[w L+P X]\}=P_{0} M_{0}+g \sum_{k=0}^{t-1} P_{k} M_{k}
$$

Ecuación que se transforma en:

$$
P Y=(1+g)[w L+P X]+\frac{1}{t} \times\left\{1-\prod_{k=1}^{k=t}\left(1-a_{k}\right)\right\} P_{0} M_{0}+\frac{g}{t} \times \sum_{k=0}^{t-1} P_{k} M_{k}
$$

Y no olvidemos que los $\boldsymbol{P}_{k} \boldsymbol{M}_{k}$ son vectores, de tal forma que la expresión anterior se convierte en:

$$
\sum_{i=1}^{n} p_{i} y_{i j}=(1+g)\left[w l_{j}+\sum_{i=1}^{n} p_{i} x_{i j}\right]+\frac{1}{t} \times\left\{1-\prod_{k=1}^{k=t}\left(1-a_{k}\right)\right\} \sum_{h=1}^{s} p_{o h} m_{o h j}+\frac{g}{t} \times \sum_{k=0}^{t-1} \sum_{h=1}^{s} p_{k h} m_{k h j}
$$


Si hiciéramos que las tasas de depreciación $\boldsymbol{a}_{\mathrm{k}}$ fueran todas iguales, la ecuación (10.55) se convertiría en la ecuación que ya vimos en (10.30):

$$
P Y=(1+g)[w L+P X]+\frac{2\left[1-(1-a)^{t}\right]}{t} \times P_{0} M_{0}
$$

$$
P=(1+g)[w L+P X] Y^{-1}+\frac{2\left[1-(1-a)^{t}\right]}{t} \times P_{0} M_{0} Y^{-1}
$$

IV.f - Hay otra forma de llegar al mismo resultado que en (IV.a), pero el distinto camino emprendido creo que tiene algún punto de interés, razón por la cual la exponemos a continuación. Partimos de nuevo de la ecuación:

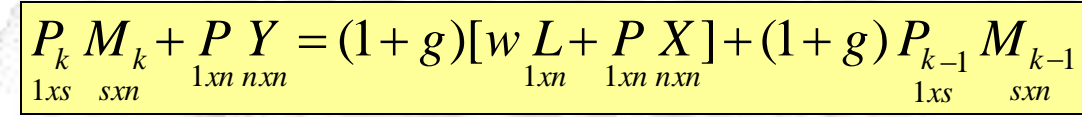

que define el modelo del capital fijo. Además, seguimos manteniendo la ecuación matricial (vectorial en este caso) que define la depreciación del capital físico (puede entenderse en términos de valor de acuerdo con una nota a pie de página ya vista) como hemos visto en el epígrafe (IV.a):

$$
P_{k} M_{k}=(1-a) P_{k-1} M_{k-1}
$$

Lo que ahora introducimos como novedad es que vamos a sustituir el valor del vector resultante $\boldsymbol{P}_{\mathrm{k}-1} \boldsymbol{M}_{\mathrm{k}-1}$ en (10.15) y queda:

$$
P_{k} M_{k}+P Y=(1+g)[w L+P X]+(1+g)\left[\frac{1}{(1-a)} \times P_{k} M_{k}\right]
$$

Y manipulando términos queda:

$$
P Y=(1+g)[w L+P X]+\frac{a+g}{1-a} \times P_{k} M_{k} \quad \forall k=1 \quad a t
$$

Y en lo vemos que cuanto mayor sea la depreciación, dado los valores físicos de $\boldsymbol{Y}, \boldsymbol{X}, \boldsymbol{L}$, aumenta exponencialmente el valor del precio $\boldsymbol{P}$ de los bienes del circulante. También aumentan los precios cuanto mayor sea la tasa de 
ganancia $\mathbf{g}$, pero este aumento es solo proporcional, lo cual evidencia el distinto peso de la tasa de financiación del capital fijo -que Sraffa toma como la tasa de ganancia- de la tasa de depreciación. De hecho en la ecuación anterior, si la tasa de depreciación a se acercara al $100 \%$, los precios $\boldsymbol{P}$ del circulante llegarían al infinito, lo cual demuestra la importancia en las empresas de alargar los bienes duraderos lo máximo posible. Al menos en este modelo de inspiración esrafiana. Demuestra también este modelo que Sraffa no estuvo muy acertado al tomar la tasa de ganancia como el bálsamo de Fierabrás, es decir, una tasa que servía para al menos tres cosas: determina la tasa de ganancia de la empresa, determina la tasa de financiación del capital fijo y determina (implícitamente al menos) la tasa de depreciación del capital fijo. No parece aceptable. Si a eso le añadimos la imposibilidad en casi todos los modelos de depreciación del capital fijo en Sraffa de hacer cero el valor de la chatarra, vemos que no estuvo muy acertado al final con el capital fijo. Sabemos que este tema le trajo de cabeza este tema a Sraffa y a los matemáticos que le asesoraron. Queda pendiente de investigación por los historiadores del análisis económico las idas y venidas, las vueltas que le dio a esto y como creo que lo que dejó no es satisfactorio. Lo importante, no obstante, es que sus errores tienen solución y, gracias al genio del turinés y a pesar de lo anterior, podemos desarrollar una teoría del capital fijo que sirve como teoría empresarial de la depreciación de este medio de producción. Volviendo a la ecuación (10.59), vamos a dar valor a $\mathbf{k}$ (vida útil del capital fijo) desde 1 a $t$ de tal forma que obtendremos las ecuaciones:

(10.60)

$$
P Y=(1+g)[w L+P X]+\frac{a+g}{1-a} \times P_{1} M_{1}
$$

$$
P Y=(1+g)[w L+P X]+\frac{a+g}{1-a} \times P_{2} M_{2}
$$

$$
P Y=(1+g)[w L+P X]+\frac{a+g}{1-a} \times P_{t} M_{t}
$$

Sumando las t ecuaciones vectoriales de (10.60) sale:

$$
P Y=(1+g)[w L+P X]+\frac{1}{t} \times \frac{a+g}{1-a} \times \sum_{k=1}^{k=t} P_{k} M_{k}
$$

Traemos ahora la relación entre el valor del capital fijo dado en el período $\boldsymbol{k}$ y el mismo capital del período $\mathbf{k - 1}$ dado por (10.24), con lo que queda: 


$$
P Y=(1+g)[w L+P X]+\frac{1}{t} \times \frac{a+g}{1-a} \times\left\{(1-a)+(1-a)^{2}+\ldots+(1+a)^{t}\right\}
$$

Reduciendo términos queda al final:

$$
P Y=(1+g)[w L+P X]+\frac{1}{t} \times \frac{a+g}{1-a} \times(1-a)\left\{1+(1-a)+(1-a)^{2}+\ldots+(1+a)^{t-1}\right\}
$$

Y la expresión que hay entre corchetes en el lado derecho de la igualdad es la suma de los términos de una progresión geométrica de razón (1-a), de primer termino 1 y de número de términos $\boldsymbol{t}$. El resultado es el mismo que el obtenido en (IV.a):

$$
P Y=(1+g)[w L+P X]+\frac{(a+g)\left[1-(1-a)^{t}\right]}{a t} \times P_{0} M_{0}
$$

Ahora vamos a despejar los precios $\mathbf{P}$ del circulante en (10.29) y queda:

$$
P=\left\{(1+g) w L+\frac{(a+g)\left[1-(1-a)^{t}\right]}{a t} \times P_{0} M_{0}\right\}[Y-(1+g) X]^{-1}
$$

Y teniendo en cuenta que $\boldsymbol{X}=\boldsymbol{A} \boldsymbol{Y}$, la expresión que hay entre corchetes cambia de taliforma que el vector de precios $\boldsymbol{P}$ se convierte en:

$$
\left.P=\left\{(1+g) w L+\frac{(a+g)\left[1-(1-a)^{t}\right]}{a t}\right]_{0} M_{0}\right\} Y^{-1}\left[I+(1+g) A+\ldots+(1+g)^{n-1} A^{n-1}\right]
$$

Si hallamos la derivada primera de (10.65) respecto a la tasa de depreciación a encontramos que, mientras $\mathbf{a}+\mathbf{g}<\mathbf{1}$ es positiva, por lo que la función de precios $\boldsymbol{P}$ (en realidad un vector) es creciente. También es crecientemente creciente respecto a la tasa de ganancia $\mathbf{g}$ porque la suma de funciones del corchete del segundo miembro es exponencial, aunque ese crecimiento sea suave si la tasa de ganancia es pequeña y el número de períodos considerados $\boldsymbol{n}$ no sea muy elevado. Si definimos $\boldsymbol{B}$ como $\boldsymbol{X}=\mathbf{Y B}$ (10.65) se transforma en:

$$
P=\left\{(1+g) w L+\frac{(a+g)\left[1-(1-a)^{t}\right]}{a t} \times P_{0} M_{0}\right\}\left[I+(1+g) B+\ldots+(1+g)^{n-1} B^{n-1}\right] Y^{-1}
$$


IV.g - Como último epígrafe de estos anexos vamos a dar un criterio de amortización o depreciación del capital fijo_que va a tener un carácter más general y, además, dinámico, frente a la constancia de la tasa de amortización que representa el coeficiente a y que hemos dado para $\boldsymbol{P}_{k} \boldsymbol{M}_{k}=(\mathbf{1}-\boldsymbol{a}) \boldsymbol{P}_{k-1} \boldsymbol{M}_{k-1}$. Hemos interpretado que a puede representar la eficiencia constante de la que habla Sraffa, aunque el economista italiano lleva a cabo una amortización financiera sumando a los costes del circulante la anualidad correspondiente de un modelo de actualización de rendimientos futuros. Ya hemos señalado que hablar de esta eficiencia sin establecer un mecanismo de depreciación del capital fijo y dejarlo solo en amortizaciones financieras parece inadecuado cuando no contradictorio. Aquí vamos a sustituir el coeficiente a de depreciación del capital fijo por un mecanismo de disminución del valor del capital fijo donde el tiempo juego un papel importante más allá del tiempo referido a la vida útil de los capitales fijos. Para ello necesariamente debe intervenir la tasa continua de amortización física (también se emplea para la amortización financiera) y hacer que $\boldsymbol{a}=\mathbf{e}^{-r t}$, donde es la base de los logaritmos neperianos, $\boldsymbol{r}$ la tasa de decrecimiento de la amortización y $\boldsymbol{t}$ el tiempo total de la vida útil del capital fijo. Hecho esto nos queda que el valor del capital fijo de un período $\boldsymbol{k}$ de vida útil de un capital fijo está relacionado con el mismo capital fijo de un período anterior mediante la fórmula:

$$
P_{k} M_{k}=\left(1-e^{-r t}\right) P_{k-1} M_{k-1}
$$

Si ahora llevamos a cabo el proceso de reproducción del circulante junto con el capital fijo de acuerdo con la fórmula general que venimos utilizando:

$$
P_{t} M_{t}+t\{P Y-(1+g)[w L+P X]\}=P_{0} M_{0}+g \sum_{k=0}^{t-1} P_{k} M_{k}
$$

El resultado de sustituir (10.24) en (10.53) y, tras manipulaciones algebraicas elementales, es:

$$
P Y=(1+g)[w L+P X]+\frac{1+g e^{r t}}{t} \times\left\{1-\left(\frac{e^{r t}-1}{e^{r t}}\right)^{t}\right\} \times P_{0} M_{0}
$$

Y ahora despejamos los precios del circulante y queda: 


$$
P=\left\{(1+g) w L+\frac{1+g e^{r t}}{t} \times\left\{1-\left(\frac{e^{r t}-1}{e^{r t}}\right)^{t}\right\} \times P_{0} M_{0}\right\}[Y-(1+g) X]^{-1}
$$

$Y$, al igual que en el epígrafe anterior, vamos a desarrollar $[\mathbf{Y}-(\mathbf{1}+\mathbf{g}) \mathbf{X}]^{-1}$ a través de la matriz $\mathbf{B}$ que surge de $\boldsymbol{X}=\mathbf{Y B}$. El resultado es:

$$
P=\left\{(1+g) w L+\frac{1+g e^{r t}}{t} \times\left\{1-\left(\frac{e^{r t}-1}{e^{r t}}\right)^{t}\right\} \times P_{0} M_{0}\right\}\left[I+(1+g) B+\ldots+(1+g)^{n-1} B^{n-1}\right] Y^{-1}
$$

La ventaja de este sistema de amortización del capital fijo es la de que la de decrecimiento $-\boldsymbol{r}$ depende del tiempo total $\boldsymbol{t}$ de la vida útil del capital fijo.

\section{Anexo a al capítulo X:}

No quería pasar por alto el epígrafe $\mathbf{8 3}$ de $\mathbf{P M p M}$. Tenemos la ventaja en este epígrafe de que Sraffa nos presenta un dibujo y, además, nos presenta también una ecuación, aunque no la acabamos de entender. Mejor dicho, lo que no entendemos es la relación entre el dibujo y la ecuación (pag. 103 de PMpM). La propia ecuación queda incompleta al faltarle el signo de igualdad, aunque ello, por la explicación, podemos aventurarnos de lo que se trata. Tampoco queda clara el dibujo porque nos presenta en el eje de ordenadas el "valor (como proporción a la edad cero) y en el eje de abcisas lleva "la edad del instrumento duradero (t años)". Después traza varias curvas para los diferentes tipos de interés. Lo que haremos aquí es intentar llegar a la ecuación mencionada y veremos su curva. Para un bien de capital fijo cuya vida útil fuera de $\boldsymbol{n}$ años, cuyo tipo de interés fuera $\boldsymbol{r}$ y con amortizaciones constantes a lo largo del ciclo de $\boldsymbol{A}$, el valor actualizado de dicho medio de producción $\mathbf{V}_{\mathbf{n}}$ sería:

$$
V_{n}=\frac{\left[(1+r)^{n}-1\right]}{r(1+r)^{n}} \times A
$$

Por otro lado, el valor del mismo medio de capital fijo en cualquier período anterior $\mathbf{t}$ sería: 


$$
V_{t}=\frac{\left[(1+r)^{t}-1\right]}{r(1+r)^{t}} \times A
$$

Lo único que cambia, pues, es el período de tiempo considerado de vida útil en la empresa. Ahora vamos a dividir $\boldsymbol{V}_{\dagger}$ entre $\boldsymbol{V}_{\mathrm{n}}$ para que nos dé lo más parecido a la ecuación que Sraffa nos presenta, a pesar de que en el gráfico hace lo contrario. El resultado es:

$$
\frac{V_{t}}{V_{n}}=\frac{\left[(1+r)^{t}-1\right]}{\left[(1+r)^{n}-1\right] \times \frac{(1+r)^{n}}{(1+r)^{t}}}
$$

Y tras manipulaciones pertinentes queda:

$$
\frac{V_{t}}{V_{n}}=\frac{(1+r)^{n}-(1+r)^{n-t}}{(1+r)^{n}-1}
$$

Tenemos casi la ecuación de Sraffa de forma genérico. Decimos casi porque, en Sraffa, el sustrayendo del numerador lo presenta como $(\mathbf{1}+\mathbf{r})^{\dagger}$ en lugar del correcto 98 que es $(\mathbf{1}+\boldsymbol{r})^{\text {n-t}}$. O al menos bajo la interpretación expuesta. Veamos ahora el gráfico de (a.4):

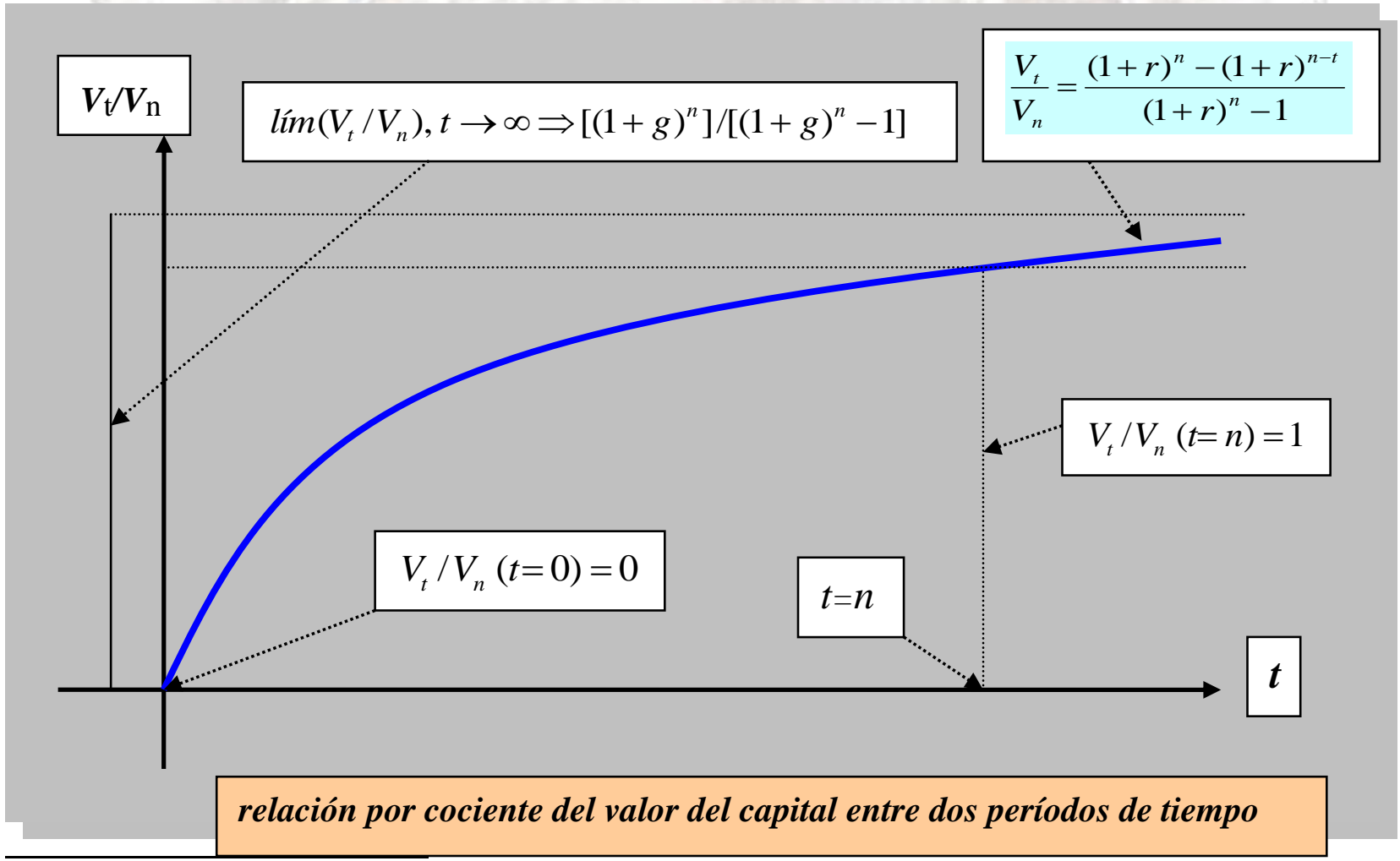

98 Lo cual lo cambia todo. 
El gráfico, por motivos obvios, no tiene sentido para valores de $\boldsymbol{t}$ mayores que $\boldsymbol{n}$. Los puntos de corte del gráfico para $\boldsymbol{t}=\mathbf{n}$ y para $\boldsymbol{t}=\mathbf{0}$ son los que se indican en el gráfico. Por otra parte la primera derivada de $\boldsymbol{V}_{\mathbf{n}} / \boldsymbol{V}_{\mathbf{t}}$ respecto a $\boldsymbol{t}$ vale $\left[(\mathbf{1}+\boldsymbol{r})^{\mathbf{n}}\right.$ $\left.{ }^{\dagger}\right] /\left[(1+r)^{n}-1\right]$, por lo que es positiva $y$, por tanto, creciente. La segunda derivada es $-\left[(\mathbf{1}+\boldsymbol{r})^{\mathbf{n}-\mathbf{t}}\right] /\left[(\mathbf{1}+\boldsymbol{r})^{\mathbf{n}-1}\right]$, es decir, negativa, por lo que es crecientemente decreciente. Por último -y aunque económicamente no tenga sentido- el límite de $\boldsymbol{V}_{\mathbf{t}} / \boldsymbol{V}_{\mathbf{n}}$ cuando $\boldsymbol{t}$ tiende a infinito es $(\mathbf{1}+\mathbf{g})^{n} /\left[(\mathbf{1}+\mathbf{g})^{n}-\mathbf{1}\right]$.

Para llegar a un gráfico parecido al de Sraffa-aunque no su ecuacióndeberemos hallar, en lugar del cociente, la diferencia entre $\boldsymbol{V}_{\mathrm{n}}-\boldsymbol{V}_{\mathrm{t}}$, es decir, la diferencia entre el valor del capital cuando se ha completado todo el ciclo del fijo y este valor para cualquier tiempo anterior del mismo (siempre $\boldsymbol{t}$ menor o igual a lo máximo de $\boldsymbol{n}$ ). Recordemos que $\boldsymbol{V}_{n}$ y $\boldsymbol{V}_{\dagger}$ son:

$$
V_{n}=\frac{\left[(1+r)^{n}-1\right]}{r(1+r)^{n}} \times A
$$

$$
V_{t}=\frac{\left[(1+r)^{t}-1\right]}{r(1+r)^{t}} \times A
$$

Ahora vamos a restar-dividirlos es lo que hemos hecho en el punto anterior-y tendremos, tras manipulaciones elementales:

$$
V_{n}-V_{t}=\frac{\left[(1+r)^{n-t}-1\right]}{r(1+r)^{n}} \times A
$$

No es esta la ecuación de Sraffa pero su gráfico sí se parece mucho a: 


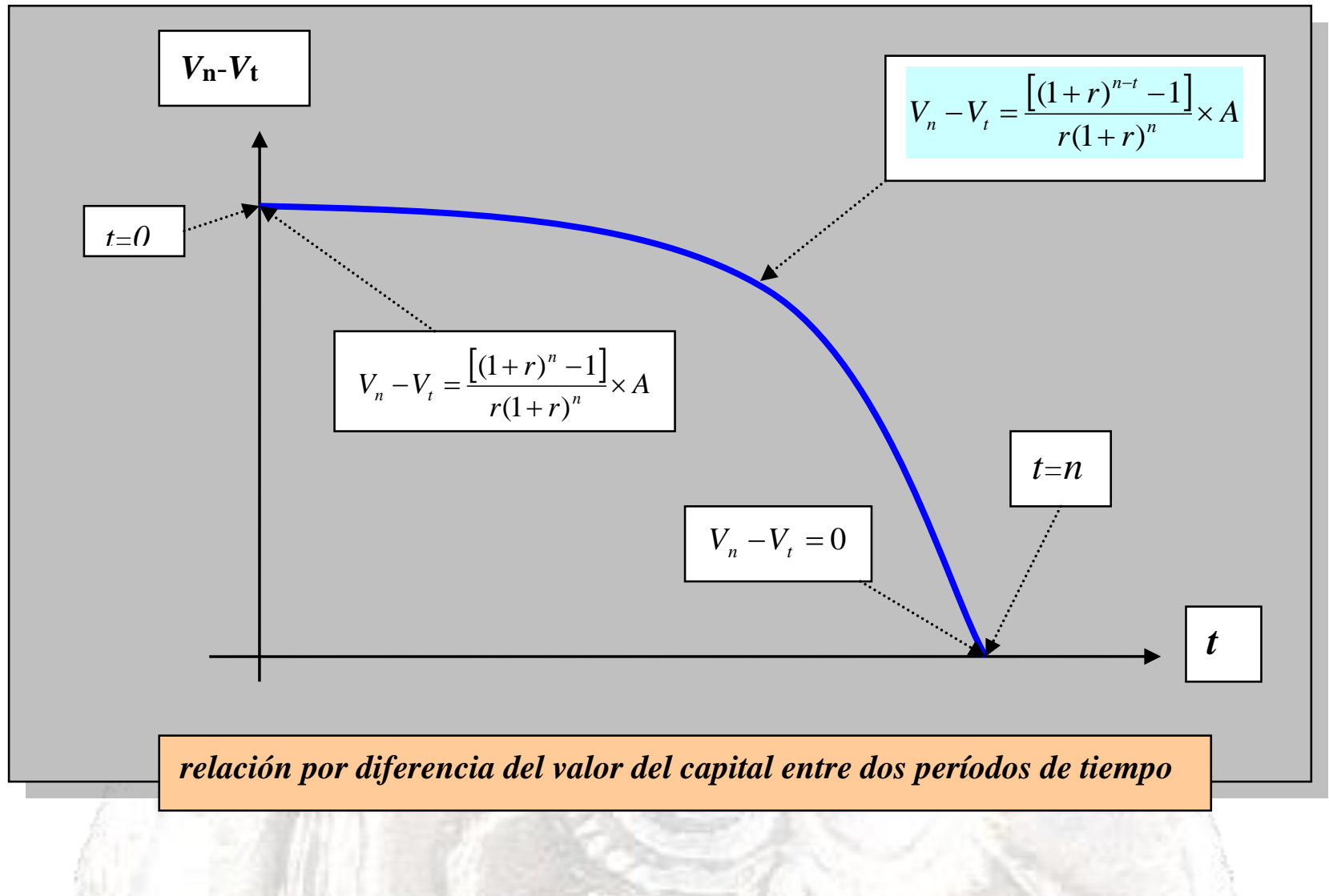

Con los puntos de corte señalados en el gráfico y decrecientemente creciente porque la primera derivada es negativa y la segunda positiva. Pero aun así la formulación no es la de Sraffa, que es:

$$
\text { Sraffa }=\frac{\left[(1+r)^{n}-(1+r)^{t}\right]}{(1+r)^{n}-1}
$$

Que no sabemos cómo la obtiene, más allá que diga que es "valor como proporción de la edad cero", según texto del gráfico en el eje de ordenadas. Dice también que "el área comprendida entre zquebrada? y los ejes representa el valor agregado de un conjunto completo de instrumentos de todas las edades". Como sabemos el área debajo de una curva es, en el cálculo diferencial, la integral de la función entre los límites escogidas en abcisas. En este caso la ecuación sería:

$$
\int_{0}^{n} \frac{1}{t} \times\left(V_{n}-V_{t}\right) d t
$$

Pero es más que dudoso que Sraffa se metiera o intentara meterse en los vericuetos del cálculo integral. Todo parece indicar que este epígrafe -el 83-es un intento fallido de Sraffa o un descuido de la formulación y/o del gráfico porque, a pesar de todo, los comentarios tienen contundencia e interés, salvo 
cuando hacen referencia al gráfico. Hasta los genios cometen errores. Creo que con esto es bastante.

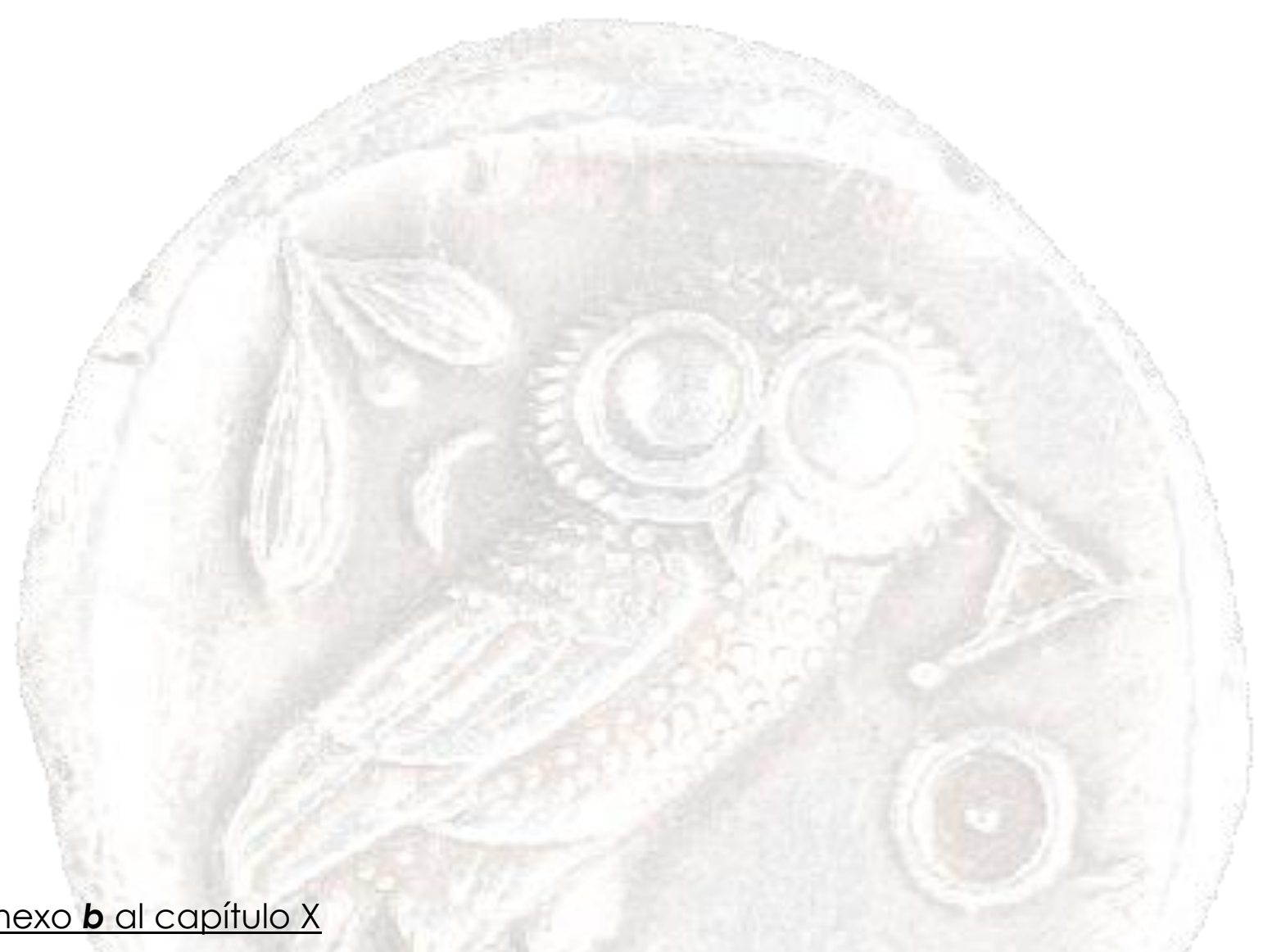

En el epígrafe $\mathbf{7 9}$ entra Sraffa en una discusión -con él mismo- sobre si las complicaciones derivadas de la producción conjunta y, en concreto, con el caso particular del capital fijo permiten "la reducción de un instrumento duradero 99 a una serie de cantidades de trabajo fechado". No se pretende en este breve -aunque parezca lo contrario- intento de desentrañar aspectos de las matemáticas explícitas e implícitas que hay en $\mathbf{P M p M}$ sustituir la casi siempre rica argumentación de Sraffa, pero en este caso no togro entenderla. Sraffa argumenta aquí que no puede efectuarse la reducción del capital fijo a trabajo fechado porque se producirían alternativamente signos negativos en los valores de $\boldsymbol{P}_{k} \boldsymbol{M}_{k}$, siendo $\mathbf{k}$ un tiempo cualquiera de la duración del capital fijo, $\mathbf{P}$ el vector de precios del capital y $\boldsymbol{M}$ un valor físico del mismo. Para ayudar a esclarecer este embrollo traemos a colación aquí la ecuación que ya vimos en este mismo capítulo.

99 Sraffa sigue evitando a toda costa usar la palabra capital. 


$$
\sum_{i=1}^{n} p_{i} y_{i j}=(1+g)\left[w l_{j}+\sum_{i=1}^{n} p_{i} x_{i j}\right]+\frac{(1+g)^{t}}{t} \times \sum_{h=1}^{s} p_{o h} m_{o h j} \quad \forall j=1 \quad a \quad n
$$

Y en términos matriciales es:

$$
P Y=(1+g)[w L+P X]+\frac{(1+g)^{t}}{t} \times P_{0} M_{0}
$$

Ya explicamos que no es esta la ecuación de Sraffa ${ }^{100}$. Eso sí, hemos dado el valor cero a la chatarra tal como quería Sraffa. A la ecuación anterior vamos a añadir la ecúación resultante de hacer cero las tasas de ganancia en (b.1) y sale:

$$
P Y=\left[w_{m} L+P X\right]+\frac{1}{t} \times P_{0} M_{0}
$$

De lo anterior despejamos los precios de la manera siguiente:

$$
P=w_{m} L Y^{-1}+P X Y^{-1}+\frac{1}{t} \times P_{0} M_{0} Y^{-1}
$$

Hemos llamado $\boldsymbol{w}_{\mathrm{m}}$, como es habitual, a la tasa máxima de salarios que surge al hacer cero la de la ganancia. Ahora sustituimos sucesivamente el vector de precios $\boldsymbol{P}$ de (b.1) en el vector de precios que pre-multiplica $a \boldsymbol{X}$ en el mismo (b.1), queda:

$$
P=\left[w L Y^{-1} \sum_{k=1}^{t}(1+g)^{k} A^{k-1}+\frac{(1+g)^{t}}{t} \times P_{0} M_{0} Y^{-1} \sum(1+g)^{k-1} A^{k-1}\right]\left[I-(1+g) A^{t}\right]^{-1}
$$

Y si en lugar de las ecuaciones anteriores hubiéramos utilizado directamente la de Sraffa para el capital fijo:

$$
P Y=(1+g)[w L+P X]+\frac{g(1+g)^{n}}{\left[(1+g)^{n}-1\right]} \times P_{0} M_{0}
$$

100 Hemos obtenido la conclusión de que no, según cómo se interprete el texto de Sraffa, no se pueden hacer simultáneamente cero la chatarra e igualar las tasas de ganancia con las de amortización. 
Si en esta ecuación, de forma análoga con lo anterior, despejamos los precios en (b.5) tal como:

$$
P=(1+g) w L Y^{-1}+(1+g) P X Y^{-1}+\frac{(1+g)^{t}}{t} \times P_{0} M_{0} Y^{-1}
$$

Y ahora vamos sustituyendo los precios $\boldsymbol{P}$ de (b.6) en el vector de precios que figura en el lado derecho de la misma ecuación, tras maniobrar convenientemente, queda:

$$
P=\left[w \sum_{k=1}^{t}(1+g)^{k} L Y^{-1} A^{k-1}+\frac{g(1+g)^{n}}{\left[(1+g)^{n}-1\right.} \times P_{0} M_{0} Y^{-1} \times \sum_{K=0}^{t-1}(1+g)^{k} A^{k}\right]\left[I-(1+g)^{t} A^{t}\right]^{-1}
$$

Cualquiera de las ecuaciones a las que hemos llegado, es decir, tanto la (b.4) y la (b.7) nos vale para intentar entender a Sraffa. En ambas ecuaciones se puede comprobar que el vector resultante $\boldsymbol{P}_{0} \mathbf{M}_{0}$ del capital fijo no se ha transformado en inputs de trabajo (este vector sólo aparece en el primer sumando pre-multiplicando a $L$ ), por lo que cabría concluir que Sraffa tiene razón y no hemos logrado reducir este capital a trabajo fechado. En cambio no se ve el segundo aspecto que señala Sraffa cual es la alternancia de los signos que debería afectar a toda la expresión donde aparece $\boldsymbol{P}_{0} \mathbf{M}_{0}$. La verdadera clave de la cuestión está en el segundo factor de ambas ecuaciones, es decir, en $\left[\mathbf{I}-(\mathbf{1}+\boldsymbol{g})^{\dagger} \boldsymbol{A}^{\dagger}\right]^{-1}$. Para que esta expresión sea positiva $-\mathrm{y}$, por lo tanto, lo sean los precios- ha de ocurrir como condición suficiente que $\mathbf{I}(\mathbf{1}+\mathbf{g})^{\dagger} \boldsymbol{A}^{\dagger}$, y dado que $\boldsymbol{A}=\boldsymbol{X}^{-1}$, tenemos que $\boldsymbol{Y}>(\mathbf{1}+\mathbf{g}) \boldsymbol{X}$, lo cual implica, con más razón que $\boldsymbol{Y}>\boldsymbol{X}$, es decir, que la economía sea productiva. Sraffa partió siempre de ese supuesto. Desde el punto de vista matemático se dice que una matriz $\boldsymbol{A}$ es productiva ${ }^{101}$ si I-A es invertible y su inversa es positiva. Es una condición necesaria y suficiente, por lo que se puede leer al revés y decir que, si la inversa de I-A es positiva, la matriz es productiva, que es tanto como decir que $\boldsymbol{Y}>\mathbf{X}$. En este caso y dado que $\boldsymbol{X}=\boldsymbol{A} \boldsymbol{Y}$, sale la inecuación $(\mathbf{I}-\boldsymbol{A}) \boldsymbol{Y}>\mathbf{I}$, por lo que $\boldsymbol{Y}>(\mathbf{I}-\boldsymbol{A})^{-1}$.

Que ni Sraffa ni aquí se haya llegado a una expresión donde el capital fijo no quede reducido a trabajo fechado, no significa que no sea cierto porque eso supondría que, según la vida útil del capital fijo (período siempre convencional), ha sido producido por medio de la mano humana directa o indirectamente o se ha producido por arte de magia con solo con máquinas, herramientas, utillaje, instalaciones, etc. Siempre hay una parte humana en la producción, traslado, implementación del capital fijo (como todo medio de producción), sea cual sea el tiempo de su consumo (depreciación). Que no se

101 Matemáticas avanzadas aplicada a la Economía, pag. 148, autores varios, UNED, 2001. 
haga explícito como señala Sraffa y se constata con las ecuaciones mencionadas, significa una limitación del modelo que estamos considerando. Significa que no lo explica todo pero, con todo, es mucho más realista y omnicomprensiva que cualquier modelo marginalista.

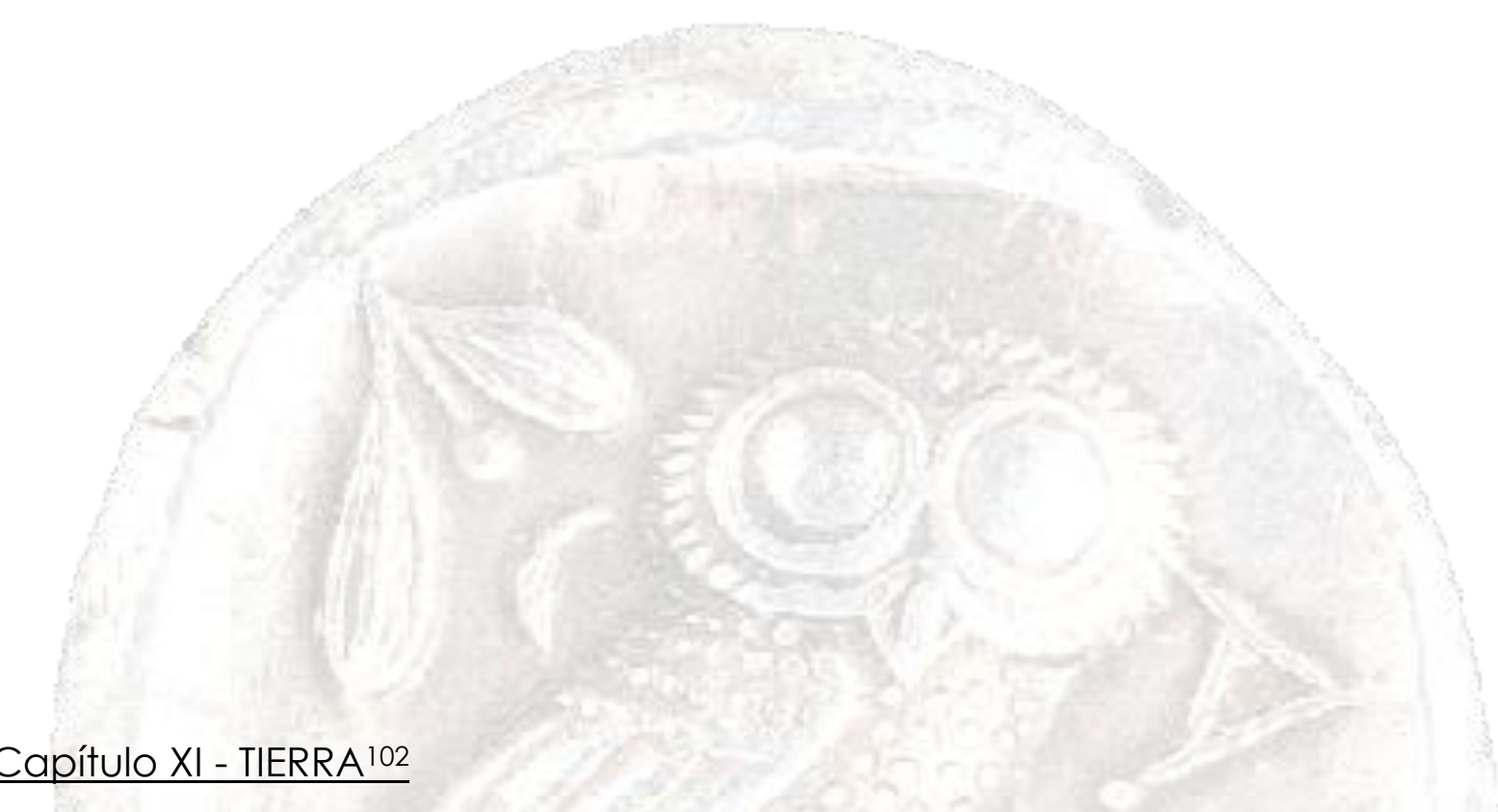

I - Abordamos ahora el capítulo XI de la obra de Sraffa que va referido al viejo tema de la renta de la Tierra. Es David Ricardo quien plantea el problema de una manera sintética y digna de un intelectual de máximo nivel como era el inglés: "Dicho cereal -se refiere en esta ocasión al maíz- no se encarece porque haya que pagar una renta, sino que debe pagarse una renta porque el cereal es caro"103. Es difícil exagerar la importancia de este texto -en general, la obra de Ricardo- y el paso que supone respecto a la consideración de Adam Smith del producto nacional como meras sumas contables de sus diferentes tipos de renta (salarios, ganancias y rentas de la tierra). Esta renta, como una de las tres retribuciones de la producción junto con los salarios (de los trabajadores) y las ganancias (de los capitalistas), la rastrea Schumpeter hasta llegar a Quesnay y a Cantillon ${ }^{104}$. A. Smith la critica y para D. Ricardo es una de las ideas-fuerza de su esquema intelectual. Ricardo la define como: "... aquella parte del producto de la tierra que se paga al terrateniente por el uso de energías originarias e indestructibles del suelo"105. No se limita a las rentas derivadas de los productos agrícolas, sino que también se refiere a las de las minas. Unos cuantos párrafos más allá, la completa, la concreta y muestra de

102 Este capítulo es el único que no es original porque se ha extraído de un libro aún no publicado titulado A partir de Sraffa, por lo que, en algún sentido, puede entenderse como original.

103 Principios de Economía y Tributación, pág. 56, FCE, edit. 1973.

104 Historia del Análisis Económico, pags. 266 y siguientes, ediciones Ariel.

105 Principios de Economía Política y Tributación, pág. 51, FCE, edit 1973. 
paso su rechazo a esta retribución cuando dice que: "Únicamente porque la tierra no es ilimitada en cantidad ni uniforme en calidad, y porque con el incremento de la población, la tierra de calidad inferior o menos ventajosamente situada tiene que ponerse en cultivo, se paga renta por su uso"106. En esta frase queda claro para el que lo lea sin prejuicios que la renta, en concreto, la de la tierra, se deriva de un problema de calidades 0 , su equivalente económicamente, de distancias. Pero es el propio Ricardo el que origina confusión cuando más tarde dice que: "Renta es siempre la diferencia existente entre el producto obtenido mediante el empleo de dos cantidades iguales de capital y de trabajo"107. Aquí parece indicar Ricardo que, aun cuando no hubiera diferentes calidades de tierra, el sólo hecho de aumentar el capital y/o el trabajo sobre la tierra origina una renta. De aquí a la ley de los rendimientos decrecientes generalizada para todos los recursos productivos y de las producfividades marginales no hay más que un paso. Para los marginalistas y neoclásicos de antes y de nuestros días, todos los factores ${ }^{108}$ se pagan - ¿o querían decir que deberían pagarse?- de acuerdo con el valor de sus productividades marginales. Pero volvamos con Sraffa y su capítulo XI y oigamos sus palabras sobre qué entiende él por renta: "Puede decirse que los recursos naturales que son utilizados en la producción, tales como la renta y los depósitos minerales, y que por ser su oferta escasa permiten a sus poseedores la obtención de una renta, ocupan entre los medios de producción una posición equivalente a la de los productos "no básicos" entre los productos"109. Puede verse por estas palabras que Sraffa no se queda en la concepción de Ricardo sobre el origen de la renta y, sin desdecir al economista inglés que tanto admiraba y al que dedicó buena parte de su vida a su obra, pone el acento el italiano -y quizá toda la partitura- en la escasez como origen de la renta. Es verdad que ya en su época era un lugar común hablar de la escasez como una de las características de los llamados fenómenos económicos y, quizá por ello, se hablaba de la economía como la ciencia lúgubre. Más tarde aclara Sraffa el porqué de ese acento: "Si no hubiera escasez, sólo se utilizaría un método, el más barato sobre la tierra, y no podría existir renta"110. En el resto de la obra Sraffa escapa del paradigma neoclásico de la escasez para llegar a su contrario -afortunadamente- de que el objeto de estudio de la economía debiera ser el estudio del excedente ocasionado por los bienes jreproducibles! No es que Sraffa lo diga así, pero así cabe deducirlo de su obra Producción de mercancías...

II - Sin más preámbulos vamos a exponer las ecuaciones, que esta vez ¡†ambién!, las hace explícitas Sraffa y que recojo en forma matricial:

\footnotetext{
106 Pag. 53 ob. citada de Ricardo.

107 Pag. 54 ob. citada de Ricardo.

108 El lenguaje se hace más aséptico y ya no se habla de trabajo, capital o tierra, sino de factores, todos en pie de igualdad.

109 Epígrafe 86 de PMPM.

110 Epígrafe 88 de PMpM.
} 


$$
\underset{1 x n}{P} \underset{n x n}{Y}=\underset{1 x n}{P} \underset{n x n}{T}+w \underset{1 x n}{L}+(1+r) \underset{1 x m m x n}{P} \underset{m}{X}
$$

donde la novedad respecto a las ecuaciones que definen el sistema esrafiano es la de la inclusión de $\boldsymbol{P}_{\dagger} \boldsymbol{T}$, siendo $\boldsymbol{P}_{\dagger}$ la renta (unitaria) de la tierra (o minas, por ejemplo) y $\boldsymbol{T}$ una matriz diagonal $\boldsymbol{n} \times \boldsymbol{n}$ que representa las cantidades de las diferentes tierras según sus calidades. La otra posible novedad es la de que los precios de los medios de producción se llevan desde $\mathbf{1}$ a $\boldsymbol{m}$, en lugar de $\boldsymbol{n}$. De momento se hace $\boldsymbol{m}=\mathbf{n}$ y en un anexo se dará la ecuación y sus posibles consecuencias para el caso más general de que $\boldsymbol{m}$ fuera diferente a $\boldsymbol{n}$. De (11.1), al hacer cero la tasa de salarios $\mathbf{w}$, obtenemos:

$$
\underset{1 x n}{\boldsymbol{P}} \underset{n x n}{\boldsymbol{Y}}=\underset{1 x n}{\boldsymbol{P}_{t}} \underset{n x n}{T}+\left(1+g_{m}\right) \underset{1 x n n x n}{\boldsymbol{P}}
$$

siendo $g_{\mathrm{m}}$ la tasa máxima de ganancia. Ahora, de (11.1) y (11.2) sale:

$$
P=\frac{w}{g_{m}-r} \times L X^{-1}
$$

donde los precios de los productos y de los medios -es decir, las no rentas- no dependen de las rentas $\boldsymbol{P}_{\uparrow} \boldsymbol{T}$. La (11.3) es muy acorde con una de las consideraciones de D. Ricardo y que hemos ya citado cuando dice que "dicho cereal no se encarece porque hay que pagar una renta, sino que debe pagarse una renta porque el cereal es caro"111. Los precios de los productos finales $\boldsymbol{P}$, sean genéricos o sea el trigo como bien final, no dependen de $\boldsymbol{P}_{\boldsymbol{t}} \boldsymbol{T}$, es decir, de las rentas. Ya queda dicho que el economista inglés, sin título universitario, era una inteligencia suprema. De (11.2) y (11.3), sustituyendo los precios de (11.3) en el lado derecho de (11.2) pero no en el lado izquierdo, queda:

$$
P_{t}=P Y T^{-1}-\frac{w\left(1+g_{m}\right)}{g_{m}-r} \times L T^{-1}
$$

Y ahora vemos como las rentas unitarias de la tierra (minas, etc.) $\boldsymbol{P}_{\dagger}$ aumentan de forma proporcional con el aumento de los precios finales $\boldsymbol{P}$, con la productividad de la tierra $\mathbf{Y T}^{-1}$, con la tasa de ganancia (interés, ganancias) $\boldsymbol{r}$ y con la tasa de ganancia máxima $\mathbf{g}_{\mathrm{m}}$ (no proporcional); disminuyen, en cambio y de forma también proporcional, con los salarios $\boldsymbol{w}$ y con la saturación de la mano de obra en relación a la tierra $\mathbf{L T}^{-1}$. De nuevo aquí las rentas (unitarias) de

111 Epígrafe 44 de PMpM. 
la tierra $\boldsymbol{P}_{\uparrow}$ dependen de los precios del producto $\boldsymbol{P}$, pero no al revés. D. Ricardo, de la mano de Sraffa, estaría satisfecho. También Pasinetti ${ }^{12}$ con su pequeño modelo ricardiano y su ecuación diferencial:

$$
R=f(N)-N \frac{d f}{d N}
$$

siendo $\boldsymbol{R}$ la renta de la tierra buscada, $\boldsymbol{f}(\boldsymbol{N})$ la función de producción (del trigo o bien final), $\mathbf{N}$ el número de trabajadores (u horas de trabajo) y $d \mathbf{f} / \mathbf{d} \mathbf{N}$ la productividad (marginal) del trabajo.

III - Una generalización del modelo esrafiano (pero con salarios prefactum) que él no se atrevió a formular podría venir dado por la ecuación:

$$
P_{1_{x m}}^{P_{m x n}}=\underset{1 x n}{P} \underset{P_{n x n}}{T}+(1+r) \times\left(w \underset{1 x n}{L}+\underset{1 x s}{P_{x}} \underset{s x n}{X}\right)
$$

donde hay $\boldsymbol{m}+\boldsymbol{n}+\mathbf{s}$ precios diferentes entre productos finales $\boldsymbol{P}_{\mathbf{y}}$, rentas unitarias $\boldsymbol{P}_{\dagger}$ y precios de los medios $\boldsymbol{P}_{\mathrm{x}}$ y con el tipo de ganancia $\boldsymbol{r}$ abarcando todos los costes. Al seguir los mismos pasos dados para deducir (4), obtenemos la ecuación:

$$
P_{t}=P_{y} Y T^{-1}-\frac{w(1+r)\left(1+g_{m}\right)}{g_{m}-r} \times L X^{T}\left[X X^{T}\right]^{-1}
$$

que no cambia nada las conclusiones de la ecuación (11.4)113. Y, al igual que en la anterior, nada nos asegura desde el punto de vista formal la no existencia de rentas negativas, lo cual no tendría sentido económico. Sólo podría evitarse al modo esrafiano acudiendo a la realidad y con los propietarios o empresarios huyendo de posibles métodos de producción -que dependen de $\boldsymbol{L}$ y $\boldsymbol{X}$ - en la agricultura que dieran lugar a semejante desaguisado.

IV - El problema de los modelos planteados, tanto del esrafiano más puro -el mencionado de Pasinetti- como el ampliado, es la de que las matemáticas tienen enorme dificultad para distinguir la calidad de la cantidad. Hemos visto que de Ricardo arrancan dos posibles interpretaciones de la renta (diferencial) de la tierra: la debida a los cambios de calidad y la del uso más intensivo del

112 Crecimiento económico y distribución de la renta, 1983, pág. 21, Alianza Editorial [Growth and Income Distribution - Essays in Economic Theory, 1974.]

$113 \mathrm{Al}$ no ser ahora $\mathbf{X}$ cuadrada se ha tenido que recurrir al cálculo de $\mathbf{X}^{\mathbf{T}}\left[\mathbf{X} \mathbf{X}^{\mathrm{T}}\right]^{-1}$ para despejar las rentas unitarias; y para que exista la inversa se ha puesto una limitación en el modelo: que $\mathbf{s}<\mathbf{n}$, es decir, que el número de medios de producción sea menor que el número de procesos. 
capital (medios, fertilizantes, etc.) con la misma tierra. A posteriori, ambas interpretaciones caben formalmente, pero las consecuencias reales son distintas por más que las matemáticas no puedan distinguirlas. Sraffa plantea el sempiterno problema de los medios de producción que no son producidos (la tierra, las minas) y la posibilidad-como es el caso del trigo- de varios métodos de producción para obtener el mismo bien final. En concreto dice Sraffa que: "Las máquinas de tipo obsoleto son similares a la tierra en la medida en que son empleadas como medios de producción aunque ya no son producidas... Y como la tierra, tales instrumentos obsoletos tienen la propiedad de los productos no básicos y son excluidos de la composición de la mercancía patrón"114. Sraffa se muestra preocupado por esa imposibilidad de obtener la mercancía-patrón, pero para obtener un modelo de economía que suponga dotar de fundamentos al análisis económico ese problema resulta irrelevante. Ya hemos visto que la conexión entre ese mundo virtual -que es la mercancíapatrón- y el modelo que define la economía real del propio Sraffa lo da la igualdad entre la tasa máxima de ganancia del modelo de la economía real y la razón-patrón, que es el excedente relativo común de todos los bienes y servicios del sistema-patrón. Pero eso ocurre sólo -hay que recordarlo- en la producción simple, que es un caso muy particular del modelo de Sraffa, aunque el propio economista italiano le dedicara mucho espacio a este modelo (producción simple).

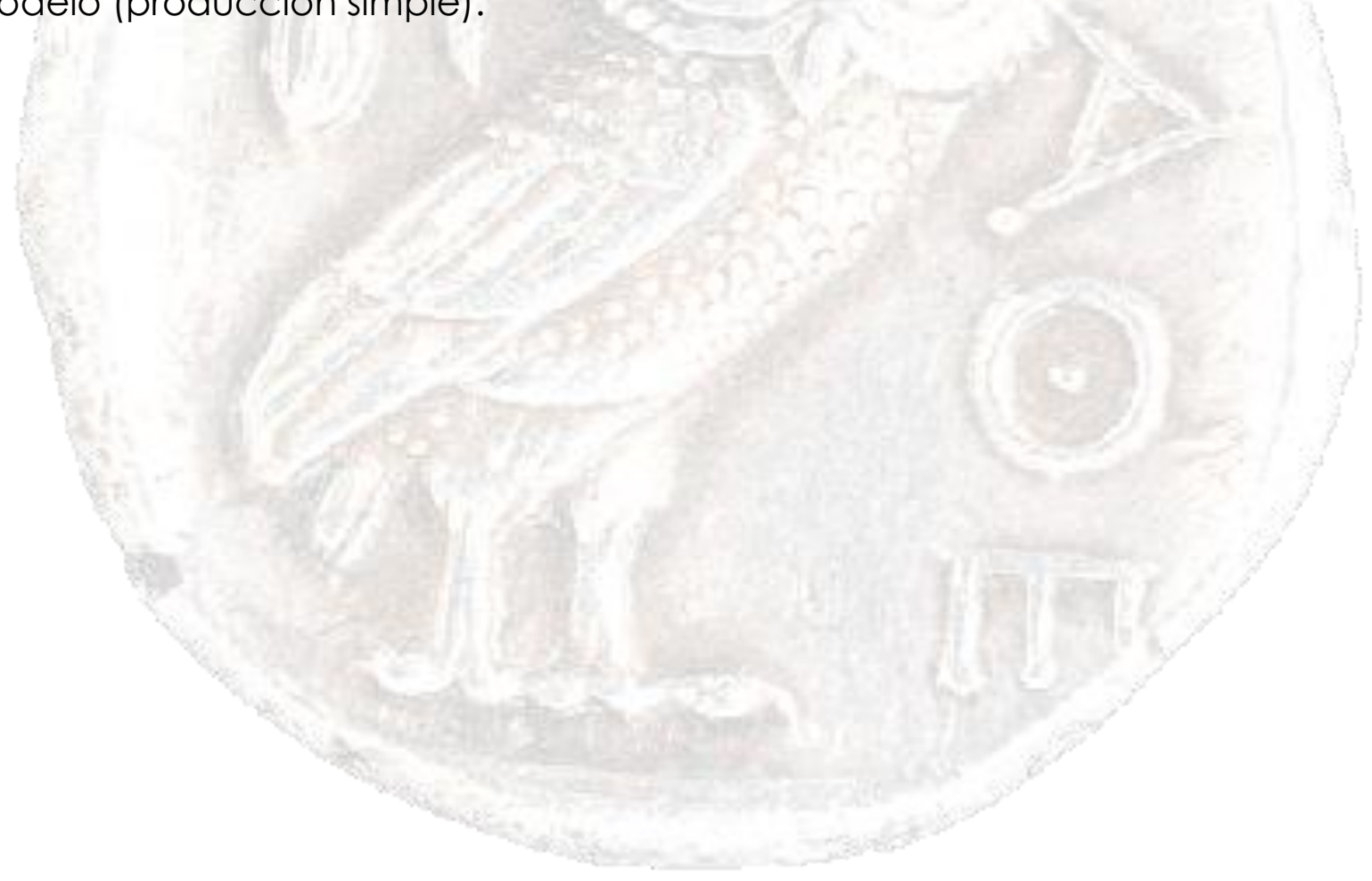

114 Epígrafe 91 de PMpM 


\section{Capítulo XII - DESPLAZAMIENTOS DE MÉTODOS DE PRODUCCIÓN}

I - Este capítulo XII que ahora tratamos podría titularse también "la teoría del capital de Sraffa", pero el economista italiano, que estuvo presente aunque sin intervenir en lo que fue la polémica sobre teoría del capital neoclásico115, lo tituló de la forma en como se encabeza este epígrafe por una razón ya expuesta: no quería que en su obra apareciera la palabra "capital". No obstante hay que señalar que el título del capítulo es muy apropiado a su contenido, pero a la altura del 2019 que es cuando está escrito este trabajo, hay que aclarar algún aspecto. En primer lugar decir que cualquier lector economista no avezado en la que fue la polémica de la teoría del capital mencionado, donde estuvieron a la gresca los dos Cambridge (el inglés y el americano) desde sus respectivas universidades, puede no advertir la importancia de esta parte del libro. Eso se debe a que Sraffa no hace mención nunca a aspectos colaterales de su trabajo teorético, como puedan ser las circunstancias históricas y los trasfondos ideológicos de sus escritos en $\mathbf{P M p M}$. Pero en este caso la tuvo y la tiene, en cierta medida, porque si aceptamos como acertado lo que se dice en este capítulo XII del libro de Sraffa, buena parte de lo que se estudian en las universidades de Economía se viene abajo. Es especial la llamada teoría del capital neoclásico y el propio marginalismo en lo que éste dependa de aquél. Esta teoría del capital viene a sostener que hay una relación siempre decreciente entre capital y trabajo 116, que ambos son sustitutos aunque sea parcialmente; lo mismo para capital y producto. Lo cual permitiría siempre también que en el mundo real las empresas puedan elegir lo que Samuelson llamó el libro de las técnicas; también y más importante, que se puedan ordenar de menor a mayor rentabilidad todas esas técnicas. No me puedo extender más y quizás ya es demasiado porque la pretensión de este trabajo es aclarar las matemáticas implícitas y explícitas que Sraffa puso en PMpM y complementarlo con algún desarrollo. Pero señalar la importancia del capítulo parecía tarea obligada, aunque esa importancia fue para Sraffa en gran medida sobrevenida. Además él nunca intervino directamente en la polémica: esa tarea se la dejó a sus discípulos, epígonos o seguidores como fueron Robinson, Garegnani, Kaldor, Pasinetti, etc 117 . Para acabar esta especie de introducción al capítulo que Sraffa, por lo dicho, ni menciona siquiera, la importancia radica en que se puede demostrar que esa pretensión de la teoría neoclásica del capital queda sencilla -pero brutalmente barrida- por este modesto -en apariencia- capítulo XII del libro. ¿Por quée Porque Sraffa demuestra que puede haber dos o más técnicas que, para un nivel de salarios, sea más rentable una de ellas, que a partir de un menor nivel de salarios (o mayor, depende desde donde se parta) pase a ser-más rentable la otra y que, a medida que bajen de nuevo los salarios jvuelva a ser más rentable la primera!, la que habíamos desechado la segunda vez. Y es importante por la

115 Conocida también por la polémica de los dos Cambridge y que el profesor Harcourt recogió en el libro mencionaddo.

116 De no ser así la teoría de la retribución de los factores en función del valor de las productividades marginales de esos factores se viene abajo, no solo empíricamente -que es incontestable-, sino también teoréticamente.

117 G. C. Harcourt recogió la polémica en 1975 en un libro: Some Cambridge controversies in the Theory of Capital. También lo hizo directamente en castellano Oscar Braun en un libro recopilatorio de artículos tituladoTeoría del capital y la distribución. 
sencillez de las hipótesis de las que se parte. En efecto, Sraffa parte de dos métodos de producción genéricos que dependen -como todo el libro de $\mathbf{P M p} \boldsymbol{M}$ - de unos medios de producción $\boldsymbol{X}$, de unos salarios $\boldsymbol{W}$ y de unos inputs de trabajo $L$, que dan lugar a unos productos $Y$ que conllevan normalmente unas ganancias g. En definitiva, la ecuación genérica tantas veces utilizada anteriormente. Es verdad que Sraffa trabaja con tasas unitarias de salarios y ganancias y que suma sin más los inputs de trabajo, pero todo esto es subsanable en su modelo teorético. En lugar de trabajo, con una sola tasa de salarios podemos trabajar con una matriz de salarios $\boldsymbol{W} n \times n$, donde cada elemento genérico $\boldsymbol{w}_{\mathrm{ij}}$ significaría, por analogía con $\boldsymbol{y}_{\mathrm{ij}}$ e $\mathbf{x}_{\mathrm{ij}}$, el salario asignado por la fabricación del producto $\boldsymbol{i}$ mediante el méłodo o proceso $\boldsymbol{j}$; en lugar de una sola tasa de ganancia $\mathbf{g}$ podemos suponer una matriz de ganancias, donde cada elemento de la matriz $\boldsymbol{g}_{\mathrm{ij}}$ representaría, análogamente, el porcentaje de ganancia que le corresponde a la empresa por la producción del elemento $\boldsymbol{y}_{\mathrm{ij}}$ mediante el proceso $\boldsymbol{j}$. Por último, los inputs de trabajo $\boldsymbol{L}$ puede ser homogeneizados mediante una matriz tal que nos de la cantidad $\boldsymbol{l}_{\mathbf{i}}$ de trabajo equivalente correspondiente al total del trabajo. Retornamos al modelo de Sraffa, pero con la seguridad formal de que lo que se dice con tasas unitarias se podría decir lo mismo tasas múltiples de tasas de ganancia y de tasas de salarios. Sea la ecuación esrafiana:

$$
P Y=(1+g)[w L+P X]
$$

Y despejando los precios, desarrollando los términos, llamando $\boldsymbol{A}$ a la matriz que surge de $\boldsymbol{X}=\boldsymbol{A} \boldsymbol{Y}$, como ya hemos visto, la ecuación (94) queda:

$$
P=(1+g) w L Y^{-1}\left[I+(1+g) A+(1+g)^{2} A^{2}+\ldots+(1+g)^{n-1} A^{n-1}\right]
$$

Ahora post-multiplicamos (12.2) por la matriz $Y$ de productos, a continuación post-multiplicamos también por el vector vertical de unos I y despejamos los salarios y:

$$
w=\frac{P Y I}{(1+g) L T^{-1}\left[I+(1+g) A+(1+g)^{2} A^{2}+\ldots+(1+g)^{n-1} A^{n-1}\right]}
$$

Vamos a utilizar el producto nacional PYI como numerario, que es tanto como dividir (12.3) entre este escalar y queda:

$$
\frac{w}{P Y I}=\frac{1}{(1+g) L T^{-1}\left[I+(1+g) A+(1+g)^{2} A^{2}+\ldots+(1+g)^{n-1} A^{n-1}\right]}
$$


A continuación Sraffa complica innecesariamente la cuestión dando entrada como actores la diferenciación entre productos básicos y no básicos. Más aún y como si nadara feliz con estas complicaciones, mezcla la ecuación que define su modelo de economía real (12.1)118 con salarios post-factum con la mercancía-patrón. Lo primero es innecesario y lo segundo inadecuado si lo que se pretende es demostrar que la teoría del capital neoclásico carece de consistencia lógica, además de irrelevancia empírica. Por ello seguimos con (12.4) y lo que haremos será definir dos métodos de producción distintos, diferenciándose ambos por utilizar como datos los inputs de trabajo L, los medios de producción $\boldsymbol{X}$ y los productos finales $\boldsymbol{Y}$. Solo serán variables los salarios -en términos del producto nacional PYI- y las tasas de ganancia. El resultado es que las posibilidades de crítica a la teoría del capital -y ya como construcción de una alternativa a ese modelo neoclásico- vendrán dadas por las ecuaciones:

$$
\frac{w}{P Y_{a} I}=\frac{1}{(1+g) L_{a} Y_{a}^{-1}\left[I+(1+g) A_{a}+(1+g)^{2} A_{a}^{2}+\ldots+(1+g)_{a}^{n-1} A_{a}^{n-1}\right]}
$$

$$
\frac{w}{P Y_{b} I}=\frac{1}{(1+g) L_{b} Y_{b}^{-1}\left[I+(1+g) A_{b}+(1+g)^{2} A_{b}^{2}+\ldots+(1+g)_{b}^{n-1} A_{b}^{n-1}\right]}
$$

En el gráfico que sigue vemos lo que analíticamente hemos descrito. A niveles superiores de salarios de $\boldsymbol{w}_{1}$ las ganancias son mayores para la técnica representada por la ecuación (12.5); a niveles de salarios comprendidos entre $\boldsymbol{w}_{1}$ y $\boldsymbol{w}_{2}$ en cambio da más ganancia la técnica definida por (12.6), pero a niveles de salarios inferiores a $\boldsymbol{w}_{2}$ jvuelve a ser más rentable la técnica definida en (12.5)! En los puntos correspondientes a los salarios $\boldsymbol{w}_{1}$ y $\boldsymbol{w}_{2}$-que se corresponden con las tasas de ganancia $\mathbf{g}_{1}$ y $\mathbf{g}_{2}$ - son puntos de corte que, traducido al análisis económico, son puntos de cambio de técnicas, es decir, en estos niveles de salarios y ganancias permitidos por ambas técnicas le sería indiferente a la empresa una técnica $u$ otra en ese momento.

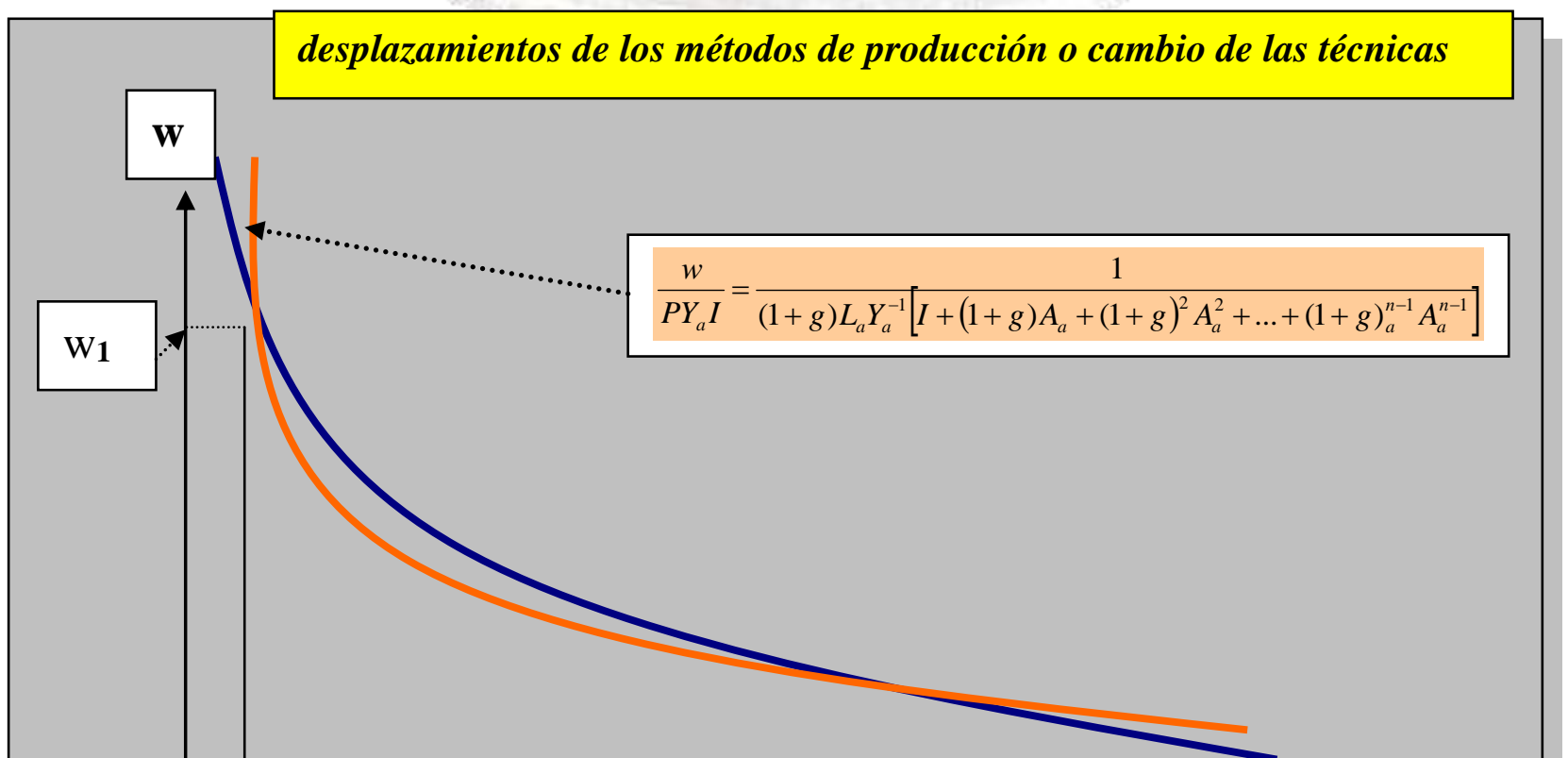




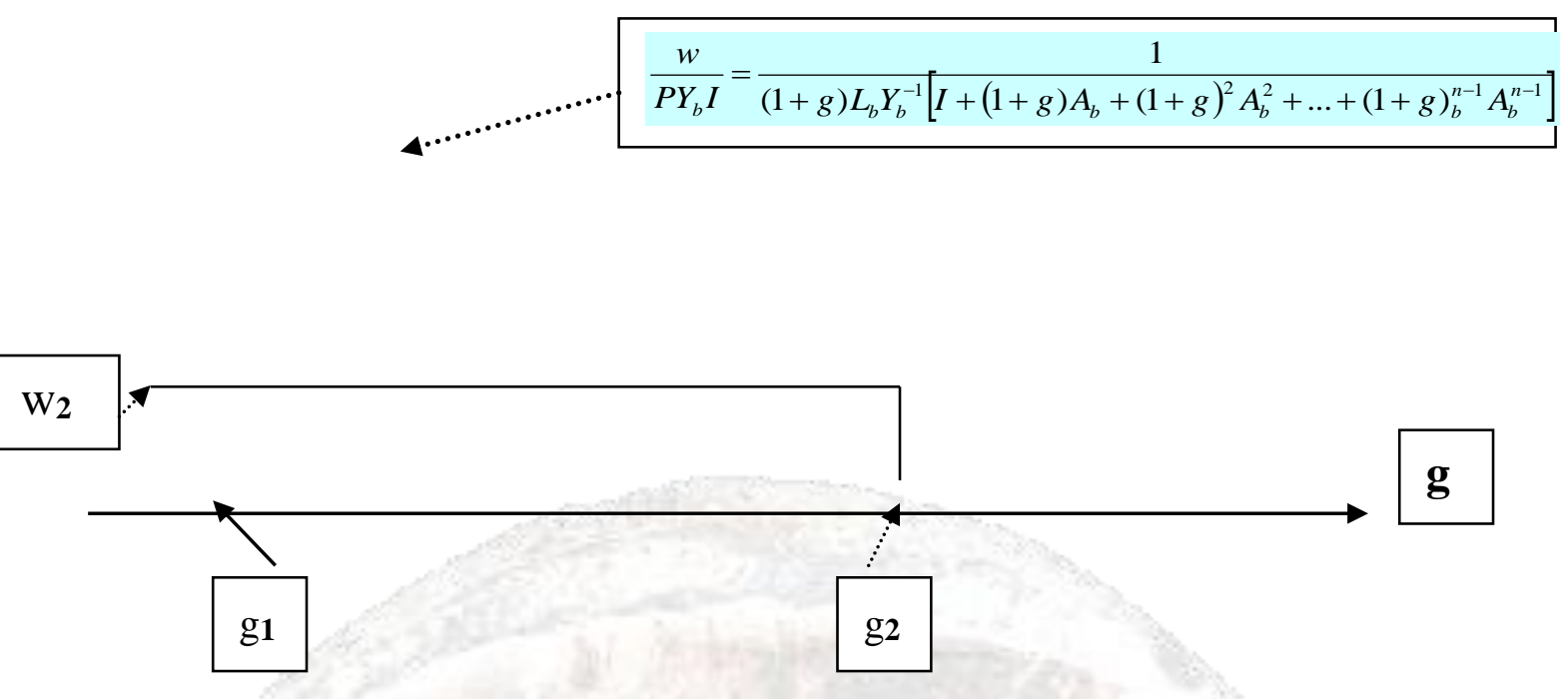

La teoría del capital neoclásica es contraria siempre a esta posibilidad porque de admitirla, no tendría una retribución de los factores-empleamos su lenguaje- que fuera dependiente de lo que llaman valor de la productividad marginal del trabajo y del capitall19. Lo escalofriante de esta refutación de la teoría neoclásica es el modelo del que se parte. En efecto, es difícilmente rebatible una economía pueda ser mejor descrita -aunque sea simplificadamente- $y$, sobre todo, con menos condicionantes que por la ecuación (12.1). Si se quiere generalizar a $\boldsymbol{n} \times \boldsymbol{n}$ tasas de salarios y a $\boldsymbol{n} \times \boldsymbol{n}$ tasas de ganancia no hay dificultad alguna, y las consecuencias analíticas serían las mismas y representadas por el mismo gráfico. Además los puntos de corte pueden ser múltiples y dependientes del número de sumandos-depende de $\boldsymbol{n}$ que hay entre corchetes de la ecuación (12.4).

II - Sraffa complica la cuestión al especular sobre las diferentes situaciones que se dan si se parte de la diferenciación entre bienes básicos y no básicos, diferenciación que no explicamos porque ya lo hemos hecho en capítulos anteriores. No obstante, por la naturaleza de intenciones de este trabajo, damos la ecuación (12.4) que hay implícita en las especulaciones de Sraffa sobre bienes básicos y no básicos en relación al desplazamiento de las técnicas.

$$
\left(p_{b}, p_{n b}\right)\left[\begin{array}{cc}
Y_{11} & Y_{12} \\
0 & Y_{22}
\end{array}\right]=(1+g)\left[w\left(l_{b}, l_{n b}\right)+\left(p_{b}, p_{n b}\right)\left[\begin{array}{cc}
X_{11} & X_{12} \\
0 & X_{22}
\end{array}\right]\right]
$$

donde el subíndice empleado en los precios se corresponden con los bienes básicos (b) y los no básicos (nb). Los bienes no básicos estarían representados por las matrices $\boldsymbol{Y}_{22}$ en los productos y $\boldsymbol{X}_{22}$ en los medios. Las matrices $\boldsymbol{Y}_{21}$ y $\boldsymbol{X}_{21}$

119 Para encontrar soluciones de equilibrio a su modelo el marginalismo supone que la productividad marginal de los factores (según su jerga) es siempre decreciente. 
valen cero porque es donde se ubican los bienes que serían necesarios para la producción de otros bienes distintos a ellos mismos ${ }^{120}$. Las ecuaciones que surgen de lo anterior son:

$$
P_{b}=(1+g) w L_{b}\left[Y_{11}-(1+g) X_{11}\right]^{-1}
$$

$$
P_{n b}=(1+g) w L_{n b}\left[Y_{22}-(1+g) X_{22}\right]^{-1}-P_{b}\left[Y_{12}-(1+g) X_{12}\right]\left[Y_{22}-(1+g) X_{22}\right]^{-1}
$$

Vemos que los precios (12.8) de los bienes básicos $\boldsymbol{P}_{\mathrm{b}}$ dependen solo de ellos mismos, pero los precios (12.10) de los no básicos $\boldsymbol{P}_{\mathrm{nb}}$ dependen de ellos mismo pero, también, de los básicos. Si no hubiera esos bienes que Sraffa llama -en versión L. A. Rojo- interconectados y que están representados por las matrices de producto $\boldsymbol{Y}_{12}$ y de medios $\boldsymbol{X}_{12}$, (12.9) se convertiría en:

$$
P_{n b}=(1+g) w L_{n b}\left[Y_{22}-(1+g) X_{22}\right]^{-1}
$$

Y lo representado ahora por (12.8) y (12.9), no es una economía, sino dos economías sin relación entre ellas porque ningún bien de la economía representada por $\left(\boldsymbol{Y}_{11}, \boldsymbol{X}_{11}\right)$ se utiliza como medio en la representada por $\left(\boldsymbol{Y}_{22}, \boldsymbol{X}_{22}\right)$ y viceversa. Serían dos economías -dos países- que no mantienen ninguna relación -al menos comercial- entre sí. El comercio estaría representado por parte de las matrices $\left(\boldsymbol{Y}_{12}, \boldsymbol{X}_{12}\right)$.

Volviendo al tema principal de este capítulo, obviando la diferenciación entre bienes básicos y no básicos y obviando, también, la mercancía-patrón, hay que ponderar la importancia de este capítulo, que es breve (7 páginas en la traducción española), pero trascendente en lo teorético y en la historia del análisis económico. O así debiera serlo. Es cierto que en la economía real los cambios de las técnicas o desplazamientos en los métodos probablemente no sean muy frecuentes. Es posible que la mayor parte del cambio de estas técnicas no se corten nunca o se corten una vez (lo cual ya va contra el modelo neoclásico), pero estamos hablando de fundamentos del análisis, no de leyes sociológicas -la economía o es una sociología o solo quedan fórmulas y gráficos que nada significan- y los fundamentos no pueden admitir excepciones. O al menos deber ser así si queremos un análisis económico con fundamentos. Quizás no tengamos que resignar los economistas a aceptar leyes cualitativas, pero no se pueden renunciar a ellas desde los fundamentos. Además existe una razón histórica del porqué los economistas considerados

120 La matriz $\mathbf{X}_{22}$ se emplea solo en producir $\mathbf{Y}_{22}$ en todo caso y nunca en la producción de los bienes ubicados en $\mathbf{Y}_{11}$. En cierto modo se presenta las paradojas clásicas de la lógica de la autoreferencia. En cierto modo los bienes representados por $\left(\mathbf{Y}_{22}\right.$ y $\left.\mathbf{X}_{22}\right)$ también son básicos para ellos mismos, a diferencia de los que están en $\left(\mathbf{Y}_{11}, \mathbf{X}_{11}\right)$ que son básicos porque se producen a sí mismos pero también a los que se ubican en $\mathbf{Y}_{12}$. 
clásicos aceptaron la teoría del capital y es la de que hasta Sraffa y Leontief no se consideraban las relaciones intersectoriales formando parte de los fundamentos del análisis. Incluso Keynes y Kalecki trabajaron con funciones agregadas. Y no es que no hubiera precedentes históricos, porque ahí estaban los fisiócratas del siglo XVIII, pero los economistas clásicos -la mayor parte ingleses- se olvidaron de los economistas continentales -la mayor parte de los fisiócratas eran franceses- y siguieron un rumbo no estructural del análisis económico. También existieron y existen consideraciones ideológicas sobre las que no vamos a entrar. Leontief desde el mero empirismo y Sraffa sin intervenir en los debates, tiraron por la borda intelectual la teoría del capital neoclásico $y$, con ello, gran parte del marginalismo (al menos el lado de la oferta). Al considerar las relaciones intersectoriales ya no se dan necesariamente unas relaciones inversas entre productividades marginales de los factores y sus retribuciones, entre las relaciones de capital y el producto o entre el mismo capital y el trabajo: no siempre estas relaciones tienen el mismo signo, la misma relación, aunque sean mayoritarias las de un signo determinado (el mismo Sraffa criticó este dogmatismo en sus artículos de 1925 y 1926). Esta falta de constancia o de seguridad se debe a que los efectos indirectos de unas variables -la inversión, el gasto, el consumo- pueden ser muy distintos según sea la estructura económica de la sociedad, según sean los efectos sobre el empleo. Y, a pesar de todo, se sigue estudiando en las facultades la microeconomía como si Sraffa, Leontief, los fisiócratas, etc. no hubieran existido. Y aquí acabamos estas consideraciones.

\section{Apéndice B ${ }^{121}$ (Sraffa) - Productos no básicos que se autoreproducen}

Lo que Sraffa colocó en un apéndice podría muy bien ser un capítulo del libro $y$, no solo por lo que dice, sino por lo que omite. Comienza justificando el texto de la siguiente manera: "Consideremos una mercancía que entra en su propia producción en un grado desusadamente grande". Ello podría dar lugar a una tasa de ganancia muy pequeña y si, además, le consideramos un bien básico, muy bien podría ocurrir que la tasa de ganancia de los bienes no básicos no pudiera ser igual que la anterior -básico- por tres razones: porque los precios de los no básicos dependen de los básicos pero ¡lo básicos no dependen de los primeros!, porque estamos en un modelo de equilibrio que significa que el vector de precios de los medios de producción es el mismo que el de los productos, y porque estamos exigiendo tasas únicas de salarios y ganancias. $\mathrm{Si}$ no se diera alguno de estas tres hipótesis no habría problema. Y, en todo caso y dado que el mundo real es muy diferente a estas hipótesis, podría considerarse que el problema planteado por Sraffa es irrelevante. Por ejemplo, si partiéramos de una ecuación de definición del sistema que ya vimos en su momento en otro capítulo tal como:

${ }^{121}$ En este caso es así, con ese título, como aparece en PMpM. 


$$
\sum_{i=1}^{i=n} p_{i} y_{i j}=\sum_{i=1}^{i=n} l_{i}\left[w_{i j} \times\left(1+b_{i j}\right)\right]+\sum_{i=1}^{i=n} p_{i}\left[x_{i j} \times\left(1+b_{i j}\right)\right] \quad \forall j=1 \quad a \quad n
$$

$$
P Y=L[W \otimes(I+B)]+P[X \otimes(I+B)]
$$

donde los productos internos $\boldsymbol{w}_{\mathrm{ij}}\left(\mathbf{1}+\boldsymbol{b}_{\mathrm{ij}}\right)$ y $\mathbf{x}_{\mathrm{ij}}\left(\mathbf{1}+\boldsymbol{b}_{\mathrm{ij}}\right)$ lo son elemento a elemento. En este caso no habría el problema planteado por Sraffa puesto que tenemos $\boldsymbol{n} \times \boldsymbol{n}$ salarios y $\mathbf{n} \times \boldsymbol{n}$ tasas de ganancia. Si fueran estas tasas todas iguales -salarios entre sí y ganancias entre sí- pero los precios que multiplican a la matriz $\boldsymbol{Y}$ de productos fueran distintos de los que multiplican a la de medios $\boldsymbol{X}$, tampoco habría problema. Por último, si la matriz producto $X Y^{-1}$ fuera indescomponible, tampoco habría problema, salvo que no lo fuera la submatriz de bienes básicos de $\boldsymbol{X Y}^{-1}$. Damos a continuación las ecuaciones que originan el gráfico que Sraffa presenta en su libro (pag. 126 de PMpM, versión española).

$$
P Y=w L+(1+g) P X
$$

$$
P Y=\left(1+g_{m}\right) P X
$$

Si de estas dos ecuaciones eliminamos la matriz de productos finales $Y$ queda:

$$
P=\frac{w L}{g_{m}-g} \times L X^{-1}
$$

En (B.5) vemos que, si la tasa de ganancia $\mathbf{g}$ se acercara a la tasa de ganancia máxima $\mathbf{g}_{\mathrm{m}}$, los precios tenderían al infinito. Esto podría ocurrir por múltiples motivos, pero uno de ellos podría ser por la falta de competencia en los mercados de bienes que daría lugar a una economía de monopolio y oligopolios. Sraffa no vio esta posibilidad analítica a que daba lugar su modelo y se centró en un problema menor derivado de las hipófesis restrictivas que hacía. En todo caso es de esperar que la ecuación (B.5) ayude al lector a entender el gráfico de Sraffa de la pág. 126 de PMpP. El gráfico sería:

\section{$\mathbf{P}$}

$$
P=\frac{w L}{g_{m}-g} \times L X^{-1}
$$




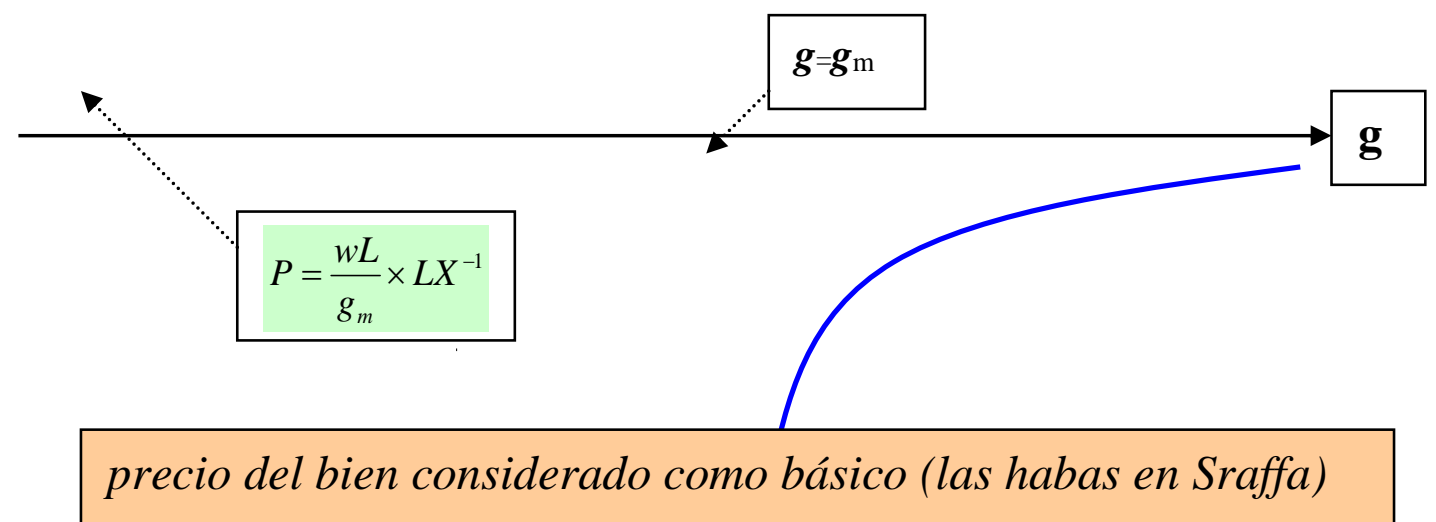

que es el mismo que expone Sraffa en este apéndice

Madrid, abril del 2019.

\section{Bibliografía}

Abraham-Frois, G y Berrebi, E. (1979): "Theory of Value, Prices and Accumulation, Cambridge U. Press (original en francés, 1976).

Afriat, S.N.: "Sraffa's Prices" (Sraffa or An Alternative Economics, 2008, Palgrave Macmillan, págs. 45-67, New York,).

Ahijado, M.: "Distribución, precios de producción y crecimiento", 1982, Centro de Estudios Universitarios Ramón Areces, Barcelona.

Ahijado, M.: "Piero Sraffa: notas para una biografía intelectual", 1985, Centro de Estudios Universitarios Ramón Areces, Barcelona.

Álvarez, A., Arándiga, M. A., Nieto, F. J., Sanz, J.: "Matemáticas avanzadas aplicadas a la Economía", 2001, UNED, Madrid.

Bandyopadhayay, P.: "Critique of Wright 2. In Defense of a PostSraffianApproach", 1981, en Steedman et al., The Valve Controversy, pags. 100129, Londres.

Barber, W.J.: "Historia del pensamiento económico", 1974, Alianza Universidad [A History of Economic Thought, 1967, Penguin Books]

Bellafiore, R y Carter, S. (2014): "Towards a new understanding of Sraffa: insight from archival research", edit. Palgrave MacMillan, New York.

Bellafiore, R.: "Sraffa after Marx" (Sraffa or An Alternative Economics, 2008, Palgrave Macmillan, pags. 68-92, New York). 
Bharadwaj, K.: "Essays on Piero Sraffa: Critical Perspectives on the Revival of Classical Theory", 2018, Routledge Library Editions.

Bidard, Ch., Poulon, F., Hendaoui, F.: "Keynes \& Sraffa", 1998, Editor: Cujas.

Bronson, R.: "Investigación operativa", 1983, serie Schaum, edit. McGraw-Hill.

Caballero, A. y Lluch, E.: "Sraffa en España", 1986, Investigaciones Económicas (2a época, vol. X, n. ${ }^{\circ}$ 2).

Carchedi, G. y W de Haan: "From Production Prices to Reproduction Prices", 1995, Capital \& Class, N 57, pags. 83-105.

Carter, S., Davis, J., Naldi, N., Lattanzi, E.. Schefold, B., Lazzarini, A. Brondino, G., Morra, L.: Research in the History of Economic Thought and Methodology, 2017, (Including a Symposium on New Directions in Sraffa Scholarship, Volume 35B), edited by Scott Carter and Riccardo Bellofiore, Editor: Emerald Publishing Limited.

Chiang, A.C: "Fundamental Methods of Mathematical Economics", 1987, McGraw-Hill; "Métodos fundamentales de la economía matemática", 1987, McGraw-Hill de México.

Chodi, G. (2008): "Sraffa or an alternative economics", edit. Palgrave Macmillan.

Chodi, G.: "Beyond Capitalism: Sraffa's Economic Theory" (Sraffa or An Alternative Economics, 2008, Palgrave Macmillan, págs.187-198, New York).

Cozzi, T., Marchionatti, R.: "Piero Sraffa's Political Economy: A Centenary Estimate" (Routledge Studies in the History of Economics), 2014, Editor: Routledge, Colección: Routledge Studies in the History of Economics.

Crespo, E. y Lazzarini, A.: "A 50 Años de "Producción de Mercancías por Medio de Mercancías" de Piero Sraffa", 2013, revista Circus, pags. 165-171, UBA, Buenos Aires.

Dobb, M.: "Teoría del valor y de la distribución desde Adam Smith", 1975, edit. Siglo XXI editores (primera edición en inglés, 1973, Cambridge U. Press). Internet: https://fundamentosdepoliticaeconomica.files.wordpress.com/2014/09/dbbma uric.pdf

Dorfman, R, Samuelson, P.A., Solow, R.M.: "Programación lineal y análisis económico", 1964, edit. Aguilar.

Dowling, E.T.: "Matemática para economistas", 1982, McGraw-Hill.

Fiorito, A.: "La implosión de la economía neoclásica", 2007, Edition: Teoría Económica, Publisher: Ediciones Cooperativas, ISBN: 978-987-1246-49-6, internet: www.geocities.com/aportexxi/sraffal2.pdf

García, N.E.: "La crisis de la macroeconomía", edit. Marcial Pons, 2010.

Garegnani, P.: "Sraffa: Análisis Clásico versus Marginalista", 2014, Revista Circus, n. 6, UBA, Buenos Aires.

Gehrke, Ch. y Salvador, N.: "Keynes, Sraffa an the Criticism of Neoclassical Theory", 2011 , edit. Routlege, New York. 
Gehrke, Ch., R. Ciccone y Mongiovi, G. (2013): "Sraffa and Modern Economics I", edit. Routledge, New York.

Gehrke, Ch.y Kurz, D.: "Sraffa on von Bortkiewicz: Reconstructing of Classical Theory of Value and Distribution" (History of Political Economy, 2006, Rocland antibodies and assays):https://www.researchgate.net/publication/31405061_Sraffa_on_von_Bor kiewicz_Reconstructing_the_Classical_Theory_of_Valve_and_Distribution

Gómez Villegas, J y Gómez-Antonio, M.: "Sobre los principios de economía. Microeconomía", 2011 , edit. Fragua, Madrid.

Hahnel, R: "Radical Political Economy: Sraffa Versus Marx", 2017, New York, N.Y, Routleged, 2017

Harcourt, G.: "ElPensamiento Económico Heterodoxo del Siglo XX", 2008, revista Circus, UBA, Buenos Aires.

Harcourt, G.C.: "Teoría del Capital", apéndice al cap. 4, 1975, edit. Oikos-tau (Some Cambridge controversies in the theory of capital, 1975).

Hodson, G.M. (1991): "After Marx and Sraffa", edit. Palgrave, Macmillan, New York.

Kubin, I.: "Market prices and natural prices: a study in the Theory of the classical process of gravitation", 1991, Land, Peter and Gmbh Internationaler Verlag Der Wissenchaften, Frankfurt.

Kurz y Salvadori (2001): "Sraffa and the mathematicians: Frank Ramsey and Alister Watson", en Piero Sraffa's Political Economy, edit Routledge.

Kurz, D. Heinz (2000): "Critical Essays on Piero Sraffa's Legacy in Economics", Cambridge University Press.

Kurz, D. Heinz: "Las contribuciones de Sraffa a la Economĺa: algunas notas sobre sus trabajos inéditos", 2007, U. N. Autónoma de México, México.

Kurz, D. Heinz: "The Hayek-Keynes-Sraffa controversy reconsidered" (Critical Essays on Piero Sraffa's Legacy in Economics, 2000, Cambridge University Press, pags. 257-304, Cambridge UK).

Kurz, D. Heinz; Salvadori, N.: "Piero Sraffa's contributions to economics: a brief survey" (Critical Essays on Piero Sraffa's Legacy in Economics", 2000, Cambridge University Press, pags. 3-24, Cambridge UK).

Kurz, D. Heinz; Salvadori, N.: "Sraffa and the mathematicians: Frank Ramsey and Alister Watson" (Piero Sraffa's Political Economy, 2001, edit Routledge, London).

Kurz, D. Heinz; Salvadori, Neri (1995): "Theory of Production", Cambridge University Press, Melbourne, Australia.

Kurz, D. Heinz; Salvadori, Neri: "Representing the Production and Circulation of Commodities in Material Terms: On Sraffa's Objetivism" (Piero Sraffa: The Man and the Scholar, 2008, Routledge, London and New York, pags. 249-278).

Kurz, H. D. y Salvatori, N.: "Sraffa y la Teoría del Valor del Trabajo", 2009, revista Circus, UBA, Buenos Aires. 
Kurz, Schefold, Salvadori: "Sraffa or an alternative economics", 2008, edt. Palmagrave Macmillan.

Hicks, J.R.: "Valor y Capital", 1945, FCE.

Lange, O.: "Political Economy, 1963, Ed. Pergamon Express, Oxford, UK (Economía Política, 1966, Ed. FCE.).

Lange, O., Taylor, F.M.: "On the Theory Economic of Socialism", 1938 (Teoría Económica del Socialismo, 1971, edit. Ariel).

Levrero, E.S.: "Some notes on wages and competition in the labour market" (Sraffa and Modern Economics, vol. I, 2011, pags. 361-384).

Levrero, S., Palumbo, A. y Stirati, A. (2006): "Sraffa and the Reconstruction of Economic Theory", revista Circus.

Lozano, E.: "Equilibrio económico general: Walras versus Sraffa", 1974, Anales de Economía, n. 21-22.

Mainwaring, L.: "Value and Distribution in Capitalist Economies: An Introduction to Sraffian Economics", 1984, Editor: Cambridge University Press.

Marchionatti Pack, S.J. (1985): "Reconstructing marxian economics: Marx based upon Sraffian commodity theory of value", edit. Praeger Publisher inc.

Menegatti, M.: "Piero Sraffa - An Italian Economist", 2008, Editor: VDM Verlag Dr. Mueller e.K.

Mora Plaza, A.: "Descifrando a Sraffa", 2011, Editorial Académica Española, Madrid.

Mora Plaza, A.: "Sraffa: lógica económica y matemáticas", 2012, publicado en la revista "Nómadas" de la U. Complutense de Madrid, (segundo semestre 2012)

http://pendientedemigracion.ucm.es/info/nomadas/36/antoniomoraplaza_2.p df

Mora Plaza, A.: "Introducción a Sraffa. Una teoría de la inflación no monetaria a partir de Sraffa", 2016, Revista de Economía, Facultad de Ciencias Económicas, Universidad Central de Ecuador, vol 68, n. 108.

Mora Plaza, A.: "La función de consumo de Keynes sobre fundamentos esrafianos", 2011, publicado en la pág. web de la U. de Málaga, (eumed.net/Contribuciones a la Economía, febrero 2011).

Mora Plaza, A.: "Modelo integrado Keynes-Sraffa", 2011, Publicado en la pág. web de la U. de Málaga, (eumed.net/Contribuciones a la Economía, marzo $2011)$.

Mora Plaza, A.: "Notas sobre fundamentos para una nueva teoría económica. Crítica de F. Azcurra y réplica del autor", 2012, revista "Nómadas" de la U. Complutense de Madrid, (primer trimestre de 2012).

Mora Plaza, A.: "Líneas posible de avance del modelo de Sraffa", 2016, Iberian Journal of the History of Economic Thought, U. Complutense de Madrid, Vol. 3, n. 2016 , pags. 42 a 67. 
Mora Plaza, A.: "Introducción elemental a Sraffa", 2018, Editorial Académica Española (LAP LAMBERT Academic Publishing GMBH).

Naldi, N.: "On the origins of Piero Sraffa equations. New evidence following Pierangelo Garegnani's lead", dec. 2018, Centro Sraffa Working Papers, n. 33, University of Roma "La Sapienza".

Nisticò S.: "Sraffa 1926 and Sraffa 1960: an attempt to bridge the gap" (Sraffa or An Alternative Economics, 2008, pags. 114-126, Palgrave Macmillan, New York).

Nisticò S; Rodano G.: "Reflections on Sraffa's Legacy in Economics: A Review Essay" (Piero Sraffa: The Man and the Scholar, 2008, Routledge, London and New York, pags. 307-325).

Pack, S.J.: "Reconstructing Marxian Economics: Marx Based Upon a Sraffian Commodity Theory of Value", 1985, Editor: Praeger Publishers Inc.

Palumbo, A. y Stirati, A. (2013): "Sraffa and the reconstruction of economic theory", edit. Levrero.

Pasinetti, L. (1980): "Essays on the Theory of Joint Production", London, edit. Macmillan, (en italiano en 1977).

Pasinetti, L. (1983): "Lecciones de teoría de la producción" [Lezioni di teoria della produzioni, 1975], FCE.

Pasinetti, L.: "Piero Sraffa and the future of economics", 2012, Cambridge Journal of Economics, 36, 1303-1314 doi: 10.1093/cje/bes036.

Peris i Ferrando, J.E . (1987): "Análisis de la resolubilidad de modelos lineales de producción conjunta", en internet: http://rua.ua.es/dspace/bitstream/10045/3829/1/Peris\%20Ferrando,\%20Josep.p df

Potier, J.P.: "Piero Sraffa", 1994, ediciones Alfons Magnànim, Valencia (Un économiste non conformiste. Piero Sraffa (1898-1883). Essay biographique, 1987, Presse Universitaires de Lyon).

Ranchetti, F.: "On the relationship between Sraffa and Keynes", en Piero Sraffa's Political Economy, edited by T. Cozzi and R. Marchoniatti, Routledge Taylor and Francis Group.

Ricardo, D.: "Principios de Economía Política y Tributación", 1973, F.C.E (On the Principles of Political Economy and Taxation, Cambridge U. Press, 1950, Londres).

Roncaglia, A.: "Piero Sraffa", 2009, Edit. Palgrave MacMillan, New York.

Roncaglia, Alessandro (1978): "Sraffa and the Theory of Prices", New York: Wiley, [Sraffa e la teoria dei prezzi, 1975].

Roncaglia, Alessandro (2009): "La riqueza de las ideas", Prensas Universitarias de Zaragoza, (The Wealth of Ideas. A History of Economic Thought, Cambridge University Press, 2005).

Roncaglia, Alessandro: "Sraffa and the Theory of Prices", 1978, New York: Wiley (Sraffa e la teoria dei prezzi, 1975, Roma-Bari). 
Salvadori, N.: "Sraffa on Demand: a textual analysis" (Critical Essays on Piero Sraffa's Legacy in Economics", 2000, Cambridge University Press, pags. 181-197, Cambridge UK).

Sánchez Choliz, Julio: "La razón-patrón de Sraffa y el cambio técnico", 1989, Investigaciones Económicas, $2^{a}$ época, Vol. XIII, internet: ftp://ftp.funep.es/InvEcon/paperArchive/Ene1989/v13ila7.pdf

Schefold, B., Bharadwaj, K.: "Essays on Piero Sraffa: Critical Perspectives on the Revival of Classical Theory": Volume 6 (Routledge Library Editions: The History of Economic Thought), 2017, Editor Routledge,

Schefold, Bertram (1989): "Mr. Sraffa on Joint Production and other essays", edit. Routlegde, New York.

Sheppard, E.: "The Capitalist Space Economy: Analysis After Ricardo, Marx and Sraffa", 1990, Editor: Routledge.

Sinha, A.: "Theories of Value from Adam Smith to Piero Sraffa", 2018, Routledge India, 2 edition

Spaventa, L.: "Apuntes de Economía Política", 1984, edit. Ariel.

Sraffa, Piero (1966): "Producción de mercancías por medio de mercancías" (Production of commodities by means of commodities, 1960), Oikos-tau ediciones.

Steedman, I.: "Marx, Sraffa y el problema de la transformación", 1985, FCE (Marx after Sraffa, 1977, London).

Subiza Martínez, B.: "Juegos matriciales y su aplicación a la teoría PerronFrobenius", U. de Alicante; http://www.ine.es/revistas/estaespa/1 12_3.pdf

Togati, T. D.: "Keynes and the Neoclasical Sythesis", ", en Piero Sraffa's Political Economy, edited by T. Cozzi and R. Marchoniatti, Routledge Taylor and Francis Group.

Vegara, J. M.: "Economía política y modelos multisectoriales", 1979, edit. Tecnos.

Vianello, F.: "La Demanda Efectiva y la Tasa de Ganancia: algunas reflexiones sobre Marx, Kalecki y Sraffa", 2006, revista Circus, UBA, Buenos Aires.

Woods, J. E.: "The Production of Commodities. An Introduction to Sraffa", 1990, edit. MacMillan, London.Abraham-Frois, G y Berrebi, E.: Theory of Valve, Prices and Accumulation, 1979, Cambridge U. Press (original en francés, 1976). 